\title{
Stability of large-scale oceanic flows and the importance of non-local effects
}

by

\author{
Hristina G. Hristova
}

Ing., Ecole Polytechnique, Palaiseau, France (2001)

M.Sc., Ecole Polytechnique, Montreal, Canada (2003)

Submitted in partial fulfillment of the

requirements for the degree of

Doctor of Philosophy in Physical Oceanography

at the

MASSACHUSETTS INSTITUTE OF TECHNOLOGY

and the

WOODS HOLE OCEANOGRAPHIC INSTITUTION

June 2009

(c) Hristina G. Hristova, 2009

The author hereby grants to MIT and to WHOI permission to reproduce and distribute publicly paper and electronic copies of this thesis document in whole or in part.

Author

Joint Program in Physical Oceanography

Massachusetts Institute of Technology

Woods Hole Oceanographic Institution

May 8, 2009

Certified by .

Michael A. Spall

Senior Scientist

Thesis Supervisor

Accepted by

Raffaele Ferrari

Chair, Joint Committee for Physical Oceanography 


\title{
Stability of large-scale oceanic flows and the importance of non-local effects
}

\author{
by \\ Hristina G. Hristova \\ Submitted to the Joint Program in Physical Oceanography - Massachusetts
Institute of Technology / Woods Hole Oceanographic Institution
on May 8, 2009, in partial fulfillment of the
requirements for the degree of
Doctor of Philosophy in Physical Oceanography
}

\section{Abstract}

My thesis covers two general circulation problems that involve the stability of largescale oceanic flows and the importance of non-local effects.

The first problem examines the stability of meridional boundary currents, which are found on both sides of most ocean basins because of the presence of continents. A linear stability analysis of a meridional boundary current on the beta-plane is performed using a quasi-geostrophic model in order to determine the existence of radiating instabilities, a type of instability that propagates energy away from its origin region by exciting Rossby waves and can thus act as a source of eddy energy for the ocean interior. It is found that radiating instabilities are commonly found in both eastern and western boundary currents. However, there are some significant differences that make eastern boundary currents more interesting from a radiation point of view. They possess a larger number of radiating modes, characterized by horizontal wavenumbers which would make them appear like zonal jets as they propagate into the ocean interior.

The second problem examines the circulation in a nonlinear thermally-forced twolayer quasi-geostrophic ocean. The only driving force for the circulation in the model is a cross-isopycnal flux parameterized as interface relaxation. This forcing is similar to the radiative damping used commonly in atmospheric models, except that it is applied to the ocean circulation in a closed basin and is meant to represent the large-scale thermal forcing acting on the oceans. It is found that in the strongly nonlinear regime a substantial, not directly thermally-driven barotropic circulation is generated. Its variability in the limit of weak bottom drag is dominated by highfrequency barotropic basin modes. It is demonstrated that the excitation of basin normal modes has significant consequences for the mean state of the system and its variability, conclusions that are likely to apply for any other system whose variability is dominated by basin modes, no matter the forcing. A linear stability analysis 
performed on a wind- and a thermally-forced double-gyre circulation reveals that under certain conditions the basin modes can arise from local instabilities of the flow.

Thesis Supervisor: Michael A. Spall

Title: Senior Scientist 


\section{Acknowledgments}

I would like to thank my advisor (Mike Spall) and my thesis committee (Joe Pedlosky, Rui Xin Huang, Fiamma Straneo, Raf Ferrari and Glenn Flierl) for their patience and guidance through the years.

I am grateful to Pavel Berloff for sharing with me his QG model that served as a base for the model used for the thermally-forced ocean simulations.

As part of my thesis work I visited Henk Dijkstra and his group at the Institute for Marine and Atmospheric Research (IMAU) at the University of Utrecht in the Netherlands. I would like to thank them for their hospitality and enjoyable stay. I would like to thank in particular Henk Dijkstra for letting me use his continuation code and answering all my questions. I greatly enjoyed working with him.

My fellow grad students made my stay in the Joint Program fly fast by. I want to thank them for being my loyal company for Buttery lunches, for the fun pet sitting experiences and cooking parties, as well as for all the other pleasant moments together.

During my time as a grad student, I had the opportunity to participate in a CLIVAR repeat hydrography cruise in the Pacific ocean. Although not directly related to my work, I found this experience highly rewarding. I met a lot of wonderful people. I would like to thank them for the nice time at sea and for keeping my interest in oceanography alive.

I would like to dedicate this thesis to my parents, for letting me be myself and follow my path, as well as to my brother and three sisters, for putting up all these years with their big sister far away from home. I wish I could share this happy moment with all of them.

I was supported through a graduate research assistantship from the National Science Foundation Grant OCE-0423975 and the Woods Hole Oceanographic Institution Academic Programs Office. 


\section{Contents}

1 Introduction 11

2 Part 1: Radiating instability of a meridional boundary current $\quad 17$

2.1 Introduction . . . . . . . . . . . . . . . . 17

2.2 The barotropic case . . . . . . . . . . . . . . . . . . . . . . . . . . . 19

2.2 .1 Formulation . . . . . . . . . . . . . . . . . . . . 19

2.2 .2 Identifying the radiating instabilities . . . . . . . . . . 21

2.2 .3 Results . . . . . . . . . . . . . . . . . . . . . . 23

2.3 The baroclinic case $\ldots \ldots \ldots \ldots$

2.3 .1 Formulation . . . . . . . . . . . . . . . . . . . . 29

2.3 .2 Energetics . . . . . . . . . . . . . . . . . . . . 31

2.3 .3 Results . . . . . . . . . . . . . . . . . . . . . . 32

2.4 Discussion and conclusions . . . . . . . . . . . . . . . 40

3 Part 2: A two-layer QG model for a thermally-forced ocean 43

3.1 Introduction . . . . . . . . . . . . . . . . . . 43

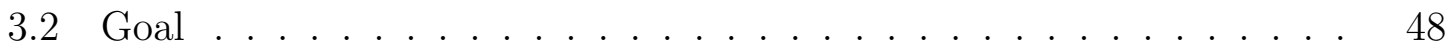

3.3 Definition of the model . . . . . . . . . . . . . . . . 48

3.3.1 The thermal forcing . . . . . . . . . . . . . . . . . 49

3.3 .2 The model equations . . . . . . . . . . . . . . 51

3.3.3 Boundary conditions and mass conservation . . . . . . . 53

3.4 Additional comments on the model . . . . . . . . . . . . . . 55 
3.4.1 Equations by vertical modes . . . . . . . . . . . 55

3.4 .2 Nondimensional equations . . . . . . . . . . . . . . 56

3.4.3 Choice of the thermal relaxation forcing . . . . . . . . 59

3.4 .4 Numerics . . . . . . . . . . . . . . . . . . . . . . . . . . . 64

4 Thermally-forced ocean in the steady regime $\quad 67$

4.1 Model setup . . . . . . . . . . . . . . . . . . . . . . 67

4.2 Overview of the circulation . . . . . . . . . . . . . . . 69

4.3 Dynamics of the barotropic circulation . . . . . . . . . . . 72

4.4 Dynamics of the baroclinic circulation . . . . . . . . . 77

4.5 Heat budget of the circulation . . . . . . . . . . . . . 81

4.5.1 The cross-isopycnal flux . . . . . . . . . . . . . 82

4.5 .2 The vertical velocity . . . . . . . . . . . . . . . 84

4.6 Summary . . . . . . . . . . . . . . . . . . . . . . . . . 87

5 Thermally-forced ocean in the time-dependent regime 89

5.1 Model setup . . . . . . . . . . . . . . . . . . . . . . . . 90

5.2 Overview of the circulation . . . . . . . . . . . . . . . . 91

5.2 .1 Time-mean circulation . . . . . . . . . . . . . . . . . . 91

5.2 .2 Temporal variability . . . . . . . . . . . . . . . . . . 94

5.2 .3 Barotropic Rossby basin modes . . . . . . . . . . . . . . . 99

5.2 .4 Main questions . . . . . . . . . . . . . . . . . . . . 104

5.3 Rectification of mean flow by the basin modes . . . . . . . . . 105

5.3.1 Mean flow driven by the basin modes . . . . . . . . . . . 105

5.3.2 Mean flow driven by an oscillating patch of wind stress . . . . 107

5.4 Baroclinic variability driven by the basin modes . . . . . . . . . . 112

5.5 Basin modes and the recirculations . . . . . . . . . . . . . . . . 119

5.5.1 Dependence of the inertial recirculations on bottom friction spatially uniform case . . . . . . . . . . . . . . . 119 
5.5.2 Dependence of the inertial recirculations on bottom friction spatially variable case . . . . . . . . . . . . . . . 125

5.5.3 Driving mechanism for the inertial recirculation gyres . . . . 129

5.5.4 Summary of basin modes and recirculations . . . . . . . . . 141

5.6 Heat budget in the time-dependent regime . . . . . . . . . . . 142

5.7 Summary . . . . . . . . . . . . . . . . . . 145

6 Onset of time-dependence in a thermally-forced ocean $\quad 147$

6.1 Introduction . . . . . . . . . . . . . . . . . . . . . 148

6.2 Problem formulation and approach . . . . . . . . . . . . 149

6.2.1 Nondimensional parameters and governing equations . . . . 149

6.2.2 Stability analysis of an equilibrium solution . . . . . . . 152

6.2.3 Perturbation energy equations . . . . . . . . . . . . . . 154

6.2 .4 Numerical methods . . . . . . . . . . . . . . . . . . 156

6.3 Stability of a thermally-forced double-gyre flow . . . . . . . . 157

6.3.1 Onset of time-dependence for $\Omega=1.2 \ldots \ldots \ldots \ldots$

6.3.2 Onset of time-dependence for $\Omega=0.3 \ldots \ldots \ldots \ldots$

6.3 .3 Hypothesis . . . . . . . . . . . . . . . . . . . 168

6.4 Stability of a wind-forced double-gyre flow . . . . . . . . . . . 169

6.4 .1 Model setup . . . . . . . . . . . . . . . . . . . . . . 169

6.4.2 Onset of time-dependence for $\Omega=1.2 \ldots \ldots \ldots \ldots \ldots$

6.4.3 Onset of time-dependence for $\Omega=0.3 \ldots \ldots \ldots \ldots$

6.5 Discussion and conclusions . . . . . . . . . . . . . . 177

$\begin{array}{lll}7 & \text { Discussion and conclusions } & 181\end{array}$

7.1 Radiating instabilities of meridional currents . . . . . . . . . . 181

7.2 Thermally-forced ocean . . . . . . . . . . . . . . . . . . 185

7.3 Baroclinic vis basin-scale instabilities . . . . . . . . . . . 186

7.4 Temporal variability dominated by barotropic basin modes . . . . . 188 
A Method of solution for the radiating instability problem

A.1 The barotropic case . . . . . . . . . . . . . . . . . . . . . . . . . . . 191

A.2 The baroclinic case . . . . . . . . . . . . . . . . . . 193

B Friction scales

C Hilbert empirical orthogonal functions analysis

$\begin{array}{ll}\text { D Perturbation energy equations } & 201\end{array}$

D.1 Derivation of the perturbation energy equations . . . . . . . . 201

D.2 Normal mode analysis . . . . . . . . . . . . . . . . . . . 204 


\section{Chapter 1}

\section{Introduction}

My thesis covers two general circulation problems, involving the stability of largescale oceanic flows and the presence of non-local effects. By non-local effects it is meant phenomena, such as radiation of Rossby waves away from a current or excitation of basin oscillations, that are caused by a localized instability but act to spread the instability influence to a much broader region. Both problems are treated in a general setting, not designed to represent any specific ocean or current. The approach undertaken instead is to use a combination of theoretical arguments and simple numerical models in order to isolate and gain understanding of the processes in an idealized setting. The basic characteristics of the phenomena can then be used to draw implications and carry comparisons with observations from different regions in the real ocean or in more complete ocean circulation models.

The first problem, presented in Chapter 2, deals with the radiating instabilities of meridional currents. An instability is said to be radiating if it has the ability through the excitation of Rossby waves to extend its influence beyond its origin region. A necessary condition for radiation is that the wavelengths and frequencies of the perturbations generated by the local instability of the current match those of the freely propagating Rossby waves in the ocean interior (McIntyre and Weissman, 1978). Radiating instabilities can be seen as a mechanism leading to the redistribution

of eddy energy in a system. Altimetry observations of eddy variability in the world 
ocean show that the majority of the eddy kinetic energy is concentrated in the regions of strong currents (Le Traon and Morrow, 2000). This suggests that the bulk of the eddy variability is due to local instabilities of the mean flow. However, as noted in Le Traon and Morrow (2000), eddy energy is present everywhere in the ocean. Radiation of Rossby waves from the source regions is one of the mechanisms that can account for the presence of eddy energy away from strong currents.

The question of energy radiation away from unstable jets has been previously looked at, but mostly in the context of zonal jets with applications to the Gulf Stream and the atmospheric circulation. For the Gulf Stream, radiating instability has been suggested as an explanation for the observed slow meridional decay of eddy energy away from the current (Talley, 1983a,b). In the context of the atmospheric circulation, radiating waves in zonally varying zonal flows have been used to explain the spatial distribution of cyclogenesis (Pierrehumbert, 1984; Finley and Nathan, 1993). Some studies on radiating instabilities have also been done of currents including a meridional component (Kamenkovich and Pedlosky, 1996; Fantini and Tung, 1987). They show that radiation is much easier if the mean flow is tilted in the meridional direction, or if, at the extreme, it flows entirely in the meridional direction.

Because of the presence of continents, nearly meridional boundary currents are widespread in the world ocean. They are present on both the eastern and the western sides of almost all basins and are often characterized by instabilities. If some of the instabilities occurring in the boundary currents are of the radiating type, then this raises the possibility that unstable boundary currents can be one of the contributors acting as a source of eddy energy for the ocean interior. Since radiation is related to the excitation of Rossby waves, which propagate energy in different zonal directions depending on their wavelength (westward for long waves and eastward for short waves), it can be anticipated that there may be some differences between the stability properties of eastern and western boundary currents. Our goal and new contribution with the work presented in this thesis is to examine systematically the stability of meridional boundary currents in both a barotropic and a two-layer baroclinic setup, 
with the particular question in mind to determine the differences in the radiating properties of eastern and western boundary currents.

The next problem, presented in Chapters 3 to 6 , deals with the dynamics of the circulation in a thermally-forced quasi-geostrophic (QG) model. A two-layer QG model can be thought of as an idealized representation of the upper warm ocean separated from the cold abyssal ocean by the thermocline. There is an extensive list of studies based on QG models on a variety of topics ranging from properties of the general ocean circulation and boundary layer dynamics (Rhines and Young, 1982; Cessi et al., 1987; Lozier and Riser, 1989; Fox-Kemper, 2003) to eddy-driven flows and internal modes of variability of the circulation (Holland, 1978; McCalpin and Haidvogel, 1996; Dijkstra and Katsman, 1997; Berloff and McWilliams, 1999a; Simonnet, 2005).

What is common for the majority of these studies, is that they consider a winddriven circulation and assume adiabatic dynamics. Therefore, effects such as sources of heat or diapicnal mixing that lead to water property transformations are neglected. Given that in the interior of the ocean, motion along isopycnal surfaces is strongly favored over motion across them, this is a good first approximation (Pedlosky, 1998). Nevertheless, cross-isopycnal fluxes play a role in setting the large-scale ocean circulation as well. The simple conceptual model for the abyssal circulation by (Stommel and Arons, 1960) is based on the idea that there is a uniform upwelling in the interior of the ocean resulting from vertical mixing, that acts in a manner similar to the Ekman pumping velocity for the ocean thermocline and drives the abyssal flow. Presence of cross-isopycnal flux is essential also when considering the question of cross-gyre flow and communication between the subtropical and subpolar gyres (Pedlosky, 1998). The scope of the second problem presented in this thesis is to examine the large-scale ocean circulation driven by a cross-isopycnal flux, representative of the large-scale thermal forcing acting on the oceans. This is done in the context of an idealized two-layer thermally forced QG model. Thus, unlike most other QG models studies, we have completely ignored the wind stress in order to focus on the large-scale ocean 
circulation driven by cross-isopycnal flux alone.

The challenge when considering a simple layer model for a thermally-forced ocean is to introduce a physically meaningful representation of the cross-isopycnal flux. One option would be to apply an externally defined spatial distribution of the crossisopycnal flux, very much like the Ekman pumping velocity is specified in models (Luyten and Stommel, 1986). However, this is a rather artificial definition, given that in reality the vertical mixing and thus the cross-isopycnal flux depend on the local stratification and small-scale turbulent processes, which are highly variable. Another option, adopted in the work presented here, is to use a parameterization of the crossisopycnal flux in terms of relaxation of the thermocline displacement to a prescribed equilibrium profile. This is commonly used in atmospheric layer models in order to represent the explicit diabatic effects due to radiative heating (Gill, 1982; Held, 2000). In the atmospheric context, in the absence of motion the vertical temperature profile of the atmosphere is determined by the solar radiation and is referred to as radiative temperature equilibrium. When the fluid is in motion, relaxing the interface to this equilibrium profile is used to model the radiative driving of the atmosphere.

In the oceanic context, we have chosen to apply the same relaxation parameterization of the cross-isopycnal flux in order to represent the large-scale thermal forcing acting on the oceans. One major difference is that the oceans, unlike the atmosphere, are not driven by radiation but by surface heat fluxes, which makes the use of the parameterization less obvious. There is thus the additional underlying assumption that the heat fluxes acting on the surface of the ocean are transmitted down the water column through vertical mixing and other processes to the thermocline, where conversion of fluid between the density layers occurs. Thus, the relaxation parameterization of the cross-isopycnal flux can be thought of as a crude representation of the vertical mixing in the thermocline. This leads to a model of the ocean circulation driven, at first look, by "internal" sources of heat, which however are a representation of the surface heating and cooling.

A linear QG model with relaxation parameterization of the cross-isopycnal flux 
has been previously used in the oceanic context in order to determine the spatial distribution of the vertical velocity resulting from surface cooling and heating in a $\beta$-plane basin (Pedlosky and Spall, 2005; Pedlosky, 2006). The new contribution of the work presented in the second part of this thesis is that we consider a model with nonlinear dynamics, where the advection of relative and stretching vorticity is included. Our goal is to study the properties of the thermally-forced circulation when the role of the nonlinear terms, as measured by the Reynolds number, is increased. We are interested in describing and understanding the time-mean large-scale ocean circulation and its variability that is driven by diapicnal fluxes at the thermocline. The element that puts this study apart from the atmospheric studies using a relaxation cross-isopycnal flux, is that in the atmospheric case the circulation in a zonally unbounded domain is normally considered, while for the ocean, we are examining the thermally-forced circulation confined to a closed basin.

The presentation of the work is as follows. In Chapter 3, the two-layer thermallyforced QG model is presented in detail and its physical meaning discussed. In Chapter 4, we examine the low Reynolds number steady regime of circulation, while in Chapter 5 the focus is on the strongly nonlinear time-dependent regime of circulation. Finally, in Chapter 6 we are interested in determining how the thermally-forced circulation transitions from steady to time-dependent behavior by performing a linear stability analysis.

One feature of the thermally-forced circulation that becomes evident, is that the variability of the circulation in the time-dependent regime is dominated by barotropic Rossby basin modes, which represent free modes of oscillation of the circulation in a closed basin. We show (in Chapter 6) that under certain conditions the basin modes can be excited from local instabilities of the mean flow. Therefore, this is another example of a non-local effect, where a local instability of the flow is able to affect a much broader region by exciting basin-scale oscillations. The majority of the analyses carried in Chapter 5 deal with establishing different consequences of the presence of variability in the form of strong barotropic Rossby basin modes. It 
is important to note however, that although we have examined the particular case of a thermally-forced ocean dominated by barotropic Rossby basin modes, all results from this Chapter can be taken in a more general context. They are likely to hold for any other situation, where strong barotropic oscillation are excited, independently of how they are driven. In other words, we are expecting that the same type of behavior can be found in a wind-driven ocean if barotropic basin modes are excited. Possible regions of interest where these results may apply, are semi-enclosed basins and marginal seas, where variability in the form of high-frequency barotropic oscillations suggestive of Rossby basin modes has been observed (Warren et al., 2002; Weijer et al., 2007a; Fu et al., 2001; Stanev and Rachev, 1999). 


\section{Chapter 2}

\section{Part 1: Radiating instability of a meridional boundary current*}

\section{$2.1 \quad$ Introduction}

Radiating instability refers to an instability of the mean flow that propagates energy away from the source of instability (McIntyre and Weissman, 1978). It can be contrasted with a trapped instability the influence of which is confined mainly to the locally unstable region and has no impact on the far field. Previous studies of radiating instabilities in the oceanic context have shown that parallel zonal eastward barotropic jets do not support radiating instabilities (Talley, 1983a,b). For these currents the perturbation energy stays trapped near the mean jet and none is radiated toward the far field. However, radiating instabilities are possible if the far field is made baroclinic or if a westward component is added to the jet (Talley, 1983a). Another way to obtain radiation is by introducing even slight non-zonality in the mean flow (Kamenkovich and Pedlosky, 1996).

A meridional current can be seen as an extreme case of non-zonality. The stability of meridional currents is less studied in the literature but is nonetheless of great inter-

*This chapter is based on the paper "Radiating instability of a meridional boundary current" by H. G. Hristova, J. Pedlosky and M. A. Spall, J. Phys. Oceanogr., vol. 38, pp. 2294-2307, 2008. 
est. Because of the presence of continents, boundary currents that are meridional or close to meridional are present on both sides of most ocean basins. Unstable boundary currents can be an important source of eddy kinetic energy. If the instabilities are radiating, then the energy of the disturbances will be transported long distances and will be able to potentially affect the mean circulation and its variability in the interior of the basin. Radiating instabilities propagate energy away from the locally unstable region by coupling to the free Rossby waves in the far field. This brings attention to a possible difference between eastern and western boundary currents. Short and long Rossby waves have different zonal directions of energy propagation so they introduce an asymmetry between eastern and western boundary currents. One can therefore anticipate different radiating properties depending on which side of the basin the current is situated on.

There are several previous studies relevant to the stability of meridional flows. In Walker and Pedlosky (2002) the baroclinic instability of a 2-layer meridional flow in a channel is examined. Compared to its zonal counterpart, the main distinction is that an arbitrarily small vertical shear leads to growing perturbations. The lack of critical threshold for linear stability is a consequence of the fact that the contributions to the mean potential vorticity gradient coming from the planetary vorticity and the mean shear are in different directions. Meridional currents are also known to have radiating instabilities. In Fantini and Tung (1987) the particular case of a meridional barotropic boundary current situated on the western side of a basin and adjacent to a motionless semi-infinite region is examined. The authors find that radiating unstable waves are generated that propagate energy eastward toward the ocean interior. The unstable waves have long meridional wavelengths and phase speeds that are larger than the speed of the jet that generates them.

The objective here is to expand our knowledge of radiating instabilities of meridional boundary currents. This is done in the context of a layered QG model on the $\beta$-plane with no dissipation. As in Fantini and Tung (1987), the boundary current is idealized by a piecewise constant profile bounded by a solid wall on one side and a 
semi-infinite motionless far field region on the other side. By solving the resulting linear stability problem, one can find whether and under what conditions the meridional current can have radiating instabilities. Compared to previous studies, emphasis is put on the differences between the stability properties of eastern and western boundary currents. Also, both barotropic and 2-layer baroclinic configurations are studied.

The plan of the presentation is as follows. Section 2.2 presents the formulation of the problem and discusses the results for the barotropic QG model. It also gives some extended discussion on the structure of the radiating instabilities. Section 2.3 deals with the stability of a purely baroclinic meridional current using a 2-layer QG model. Conclusions and physical implications are given in Section 2.4.

\subsection{The barotropic case}

\subsubsection{Formulation}

For reasons of mathematical convenience, the boundary current is idealized as a piecewise constant meridional velocity profile

$$
V=\left\{\begin{array}{ll}
V_{*} & ,|x|<x_{0} \\
0 & ,|x|>x_{0}
\end{array},\right.
$$

as in Fantini and Tung (1987). The velocity $V_{*}$ is taken positive without loss of generality. Depending on where the motionless far field is located, the flow corresponds to a western or an eastern boundary current as shown in Fig.2-1. The basic state is sustained by some large scale forcing, not specified here, since it does not appear in the linear stability problem. The departures $\psi(x, y, t)$ from the basic state are decomposed into normal modes

$$
\psi(x, y, t)=\operatorname{Re}\left\{\phi(x) e^{i m(y-c t)}\right\}
$$



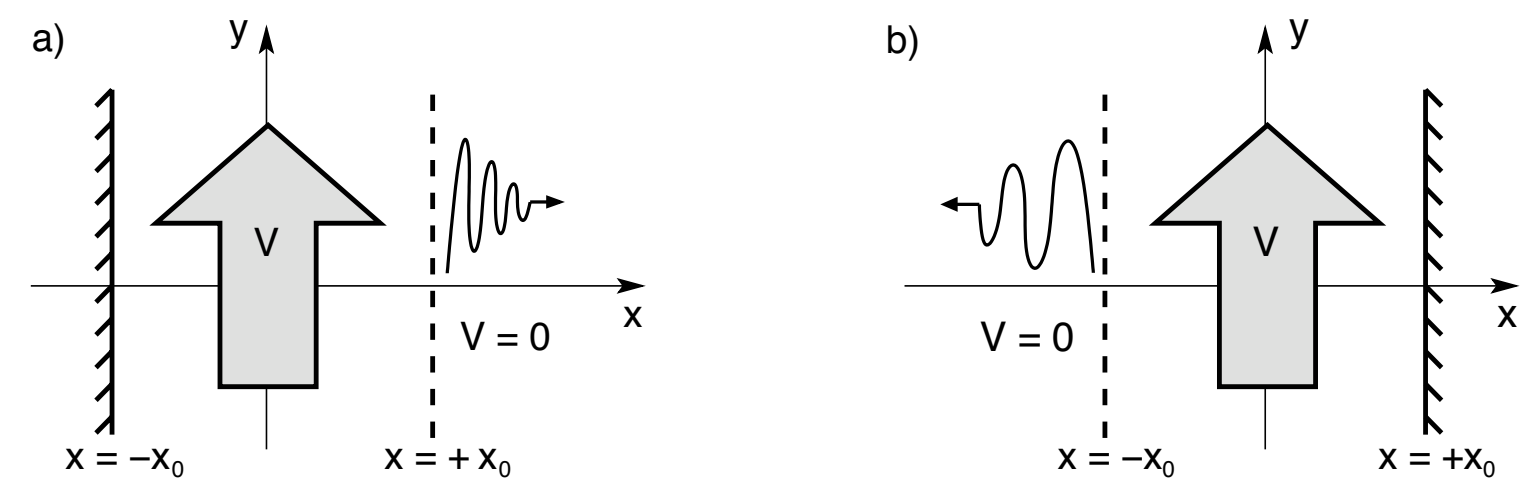

Figure 2-1: Basic state for the stability problem. Configurations for a) a western and b) an eastern boundary current.

where $m$ is the meridional (downstream) wavenumber and $c$, the phase speed in that direction. The amplitude $\phi(x)$ satisfies the linearized barotropic quasi-geostrophic potential vorticity equation

$$
(V-c)\left[\phi^{\prime \prime}-m^{2} \phi\right]+\frac{\bar{Q}_{y}}{i m} \phi^{\prime}-\bar{Q}_{x} \phi=0,
$$

where $\left\{\bar{Q}_{x}, \bar{Q}_{y}\right\}$ is the potential vorticity gradient of the basic state given by

$$
\bar{Q}_{x}=\frac{d^{2} V}{d x^{2}}, \quad \bar{Q}_{y}=\beta
$$

All variables above are non-dimensionalized using as scales the current width $L_{*}=2 x_{0}$ and the current velocity $V_{*}$. The non-dimensional planetary vorticity gradient is $\beta=\beta_{0} L_{*}^{2} / V_{*}$.

For the basic state chosen here, equation (2.3) can be further simplified since the horizontal shear and $\bar{Q}_{x}$ are identically zero. Special care has to be taken, however, of the points $x= \pm x_{0}$ where the velocity $V$ is discontinuous and $\bar{Q}_{x}$ is undefined. At these points, jump conditions derived from (2.3) hold (Kamenkovich and Pedlosky, 1996). Their role is to impose the continuity of the streamline slopes and the 
tangential pressure gradient

$$
\Delta\left[\frac{\phi}{V-c}\right]=0, \quad \Delta\left[(V-c) \phi^{\prime}+\frac{\beta}{i m} \phi\right]=0
$$

Here, $\Delta[\cdot]$ indicates the jump of the quantities in the brackets at the point $x=+x_{0}$ for a western and $x=-x_{0}$ for an eastern boundary current. The boundary condition on the other side of the current where there is a solid wall, is $\phi=0$, i.e no-normal flow.

The advantage of choosing a piecewise constant basic flow is that the stability problem (2.3) becomes a constant coefficient ODE. The amplitude $\phi$ is thus of the form $\phi \sim A e^{i k x}$, where the zonal wavenumber $k$ is related to the phase speed $c$, the meridional wavenumber $m$ and the other parameters of the problem through a dispersion relation. What is left to satisfy is the boundary and jump conditions at $x= \pm x_{0}$, the imposition of which leads to a homogeneous algebraic system. The eigenvalues $c$ are found by solving numerically the nonlinear equation that results from requiring that the determinant of the homogeneous system be zero so that there is a non-trivial solution. Once the eigenvalues $c$ are found, the solution in both the far field and the boundary current region can be reconstructed. More details on the method of solution are given in Appendix A.1.

\subsubsection{Identifying the radiating instabilities}

Suppose that for given parameter $\beta$ and meridional wavenumber $m$, we have found a value for the phase speed $c$ such that the stability problem (2.3), as well as all boundary and jump conditions are satisfied. In the far field region $\left(|x|>x_{0}\right)$ the solution is then of the form

$$
\psi(x, y, t)=\operatorname{Re}\left\{A e^{i k_{r} x} e^{i m\left(y-c_{r} t\right)}\right\} e^{-k_{i} x} e^{m c_{i} t}
$$


where the complex zonal wavenumber $k$ is related to the frequency $\omega=\mathrm{cm}$ (in general, a complex number as well) through the barotropic Rossby wave dispersion relation

$$
c m=-\frac{\beta k}{k^{2}+m^{2}} .
$$

The solution (2.6) consists of a wave with amplitude envelope that, for unstable modes $\left(m c_{i}>0\right)$, is growing in time and decaying with distance from the source. The spatial decay is a consequence of the fact that for a perturbation that is growing in time and propagating, the amplitude observed far from the source has been generated at an earlier time and is thus smaller than what is currently observed near the source. From (2.7) it follows that for each eigenvalue $c$, there are two solutions for the zonal wavenumber $k$. As shown in Fantini and Tung (1987), these two solutions have opposite signed imaginary parts $k_{i}$, as well as zonal group velocities. One of these solutions is appropriate for a western boundary current while the other, for an eastern boundary current, since the two configurations require different sign $k_{i}$ in order to have vanishing perturbation at infinity (see (2.6)). Equivalently, one can say that given the eigenvalue $c$, the far field solution consists only of the Rossby wave that has zonal group velocity away from the locally unstable region.

Because a radiating unstable solution decays into the far field very much as is expected from a trapped one, it may be confusing at first how to distinguish between the two. The distinction is however clear in the weakly unstable limit. In the limit $c_{i} \rightarrow 0$, the far field structure of a radiating solution becomes a pure Rossby wave, i.e $k_{i} \rightarrow 0$, while for a trapped solution $k_{i}$ stays finite. A mathematical expression of the statement above can be obtained from expanding the complex Rossby dispersion relation (2.7) in a Taylor series where $k_{i}$ is the small parameter, i.e. $k=k_{r}+i k_{i}$ and $\left|k_{i}\right| \ll\left|k_{r}\right|$. If only the first order term in $k_{i}$ is kept, we obtain for the eigenvalue

$$
c\left(k_{r}+i k_{i}\right)=c\left(k_{i}=0\right)+i k_{i} \frac{\partial c}{\partial k}\left(k_{i}=0\right)+O\left(k_{i}^{2}\right)
$$


The real part of (2.8) states that

$$
c_{r} \approx c\left(k_{i}=0\right)=-\frac{\beta k_{r}}{m\left(k_{r}^{2}+m^{2}\right)}
$$

In other words, in this limit the real parts of the eigenvalue $c$ and the zonal wavenumber $k$ are related through the Rossby dispersion relation. In particular, for given $\beta$ and $m$, there is a real-valued solution for $k_{r}$ only if the phase speed $c_{r}$ lies within the allowable range for barotropic Rossby wave phase speeds which is $-\beta / 2 m^{2}<c_{r}<\beta / 2 m^{2}$. For meridional phase speed $c_{r}$ that satisfies this condition, there are two possible values for $k_{r}$ that correspond to a zonally long $\left(k_{r}<m\right)$ and zonally short $\left(k_{r}>m\right)$ wave and are solutions for an eastern and a western boundary current respectively.

The imaginary part of (2.8) states that

$$
c_{i} \approx k_{i} \frac{\partial c}{\partial k}\left(k_{i}=0\right)=\frac{k_{i}}{m} c_{g}^{x}\left(k_{i}=0\right),
$$

where $c_{g}^{x}=\beta\left(k_{r}^{2}-m^{2}\right) /\left(k_{r}^{2}+m^{2}\right)^{2}$ is the zonal group velocity of free barotropic Rossby waves. It follows that the spatial decay scale in the far field $1 / k_{i}$ is proportional to the group velocity $c_{g}^{x}$ and the inverse of the growth rate $m c_{i}$. Thus, radiating unstable waves have amplitude envelopes that decay away from the source since packages of bigger and bigger amplitude are propagated at $c_{g}^{x}$ as time advances.

For practical purposes, in order to determine if an eigenmode corresponds to a radiating instability, one follows the unstable mode until it becomes marginally stable, i.e. $c_{i}=0$. If $k_{i}$ also vanishes in this limit, then the instability is radiating. The mode has a radiating wave structure in the far field roughly as long as $0<\left|k_{i}\right|<\left|k_{r}\right|$ (Fantini and Tung, 1987; Kamenkovich and Pedlosky, 1996).

\subsubsection{Results}

The only non-dimensional parameter that characterizes the barotropic problem is the $\beta$-parameter, defined previously as $\beta=\beta_{0} L_{*}^{2} / V_{*}$ where $L_{*}$ and $V_{*}$ are the current 
width and velocity. The linear stability problem defined in Section 2.2.1 is solved for the specific choice $\beta=0.5$, a typical order one value, but the results are qualitatively representative of the general behavior of the system as there is no critical value of $\beta$ for instability. When solving the eigenvalue problem, we are interested in finding the unstable eigenvalues and following them as the meridional wavenumber $m$ is varied so that we can determine whether they are radiating or not.

\section{Western boundary current}

The results are in essence the same as in Fantini and Tung (1987) except for the different choice of $\beta$. In the short wave end of the explored range of meridional wavenumbers, there is a single unstable eigenvalue (solid black line in Fig. 2-2) that asymptotes to $c=0.5+i 0.5$ when $m \rightarrow+\infty$. The lack of short meridional wave cutoff is artificial and is due to the choice of discontinuous basic state profile. When the meridional wavenumber $m$ is decreased, the growth rate $m c_{i}$ for this mode decreases and reaches zero at the critical wavenumber $m_{*}=0.355$, while its meridional phase speed $c_{r}$ increases and eventually becomes larger than one, i.e. faster than the current. Besides this mode, an additional number of unstable eigenvalues, not mentioned in Fantini and Tung (1987), are found (a representative is shown in Fig. 2-2 with a solid gray line). All these modes have $c_{r}>1$, i.e. they are faster than the current (see Fig. 2-2a). Because of the trend of $c_{i}$ to decrease to zero while $c_{r}$ goes to 1 , when the meridional wavenumber is increased, they are thought to originate in their short wave limit from the singular point $c=1$. Because of the singularity however, the point $c=1$ cannot be reached numerically and this is only assumed. Their growth rates are significantly weaker but they exist for slightly smaller values for the meridional wavenumber than the critical value $m_{*}$ (see Fig. 2-2b). Nevertheless, as concluded in Fantini and Tung (1987), it is found that there is a long meridional wave cut-off for the linear stability of a western boundary current. Hence, for meridional wavenumbers below the cut-off value, all eigenvalues have negative imaginary parts, i.e the current is linearly stable. 

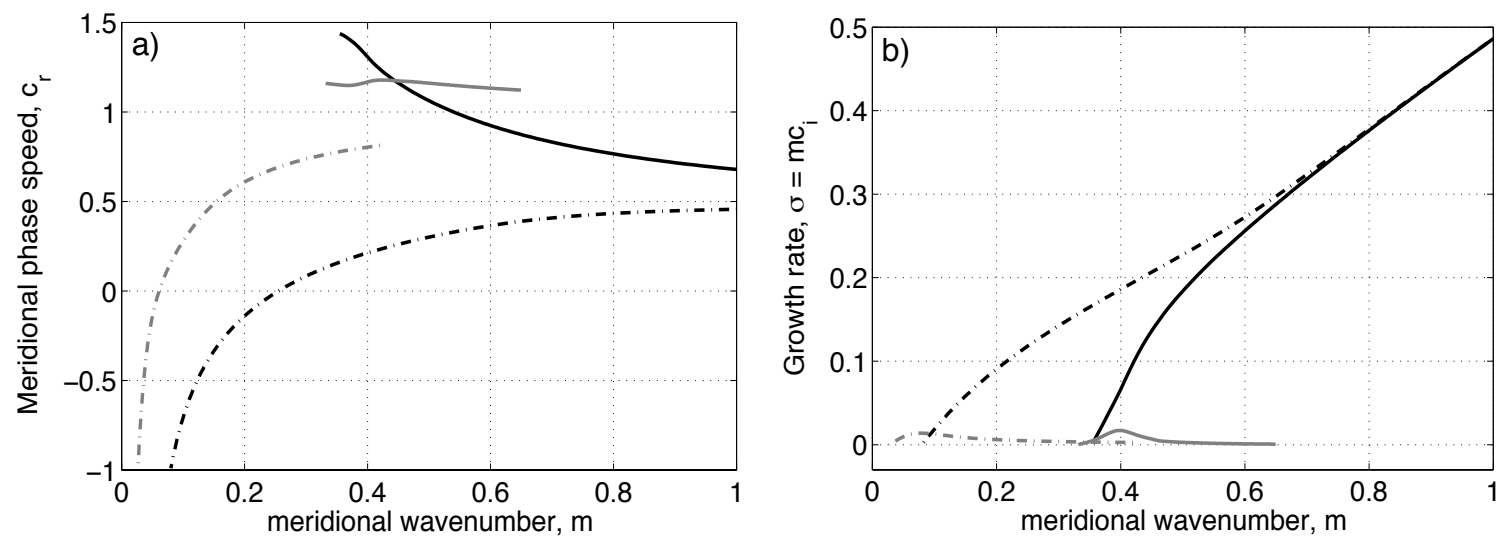

Figure 2-2: Meridional phase speed (a) and growth rate (b) as a function of the meridional wavenumber for the barotropic case with $\beta=0.5$. Solid/dot-dashed lines are used for the western/eastern boundary current. For each configuration, the most unstable eigenvalue is shown in black and the next unstable eigenvalue in gray.
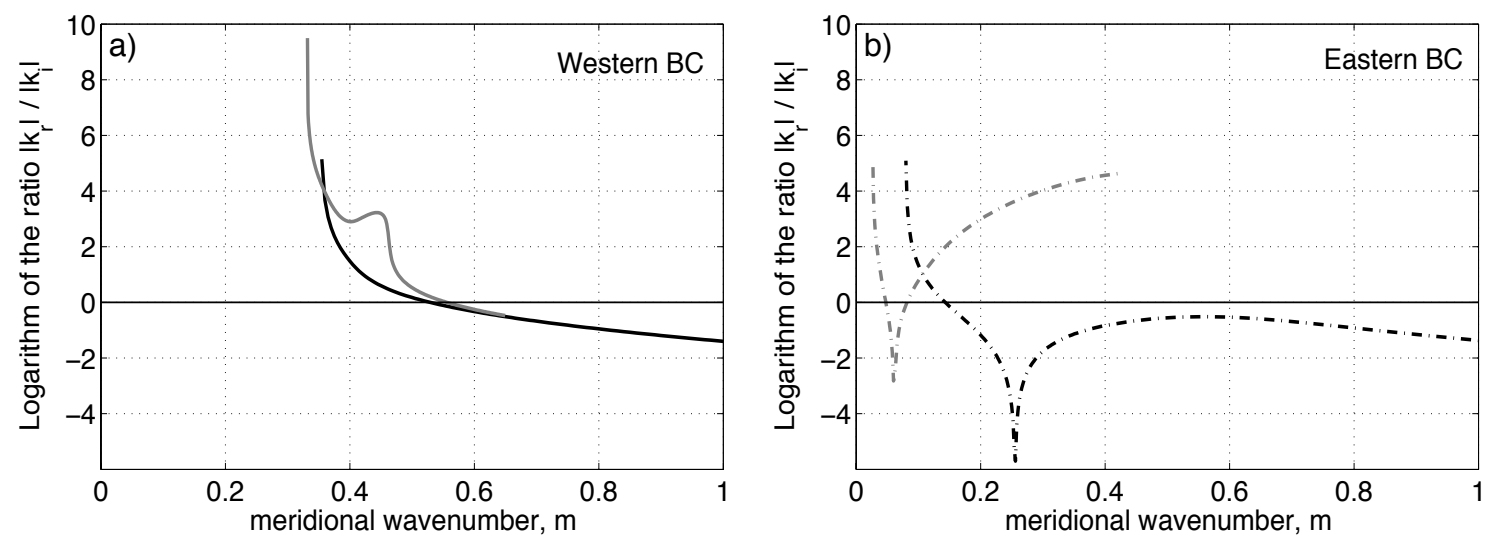

Figure 2-3: Logarithm of the ratio $\left|k_{r}\right| /\left|k_{i}\right|$ as a function of the meridional wavenumber for the western (a) and eastern (b) configurations. Here, $k_{r}$ and $k_{i}$ are the real and imaginary part of the zonal wavenumber in the far field. Same line and color code is used for the eigenvalues as in Fig. 2-2. Positive values indicate radiating wave structure.

Concerning the radiating nature of the instabilities, it is the long wave end of the explored range of meridional wavenumbers, when $c_{r}>1$, that qualifies as radiating. In Fig. 2-3a the logarithm of the ratio $\left|k_{r}\right| /\left|k_{i}\right|, k_{r}$ and $k_{i}$ being the real and imaginary part of the zonal wavenumber in the far field, is plotted as a function of $m$. For all modes, when the meridional wavenumber is decreased, the ratio $\left|k_{r}\right| /\left|k_{i}\right|$ goes to 

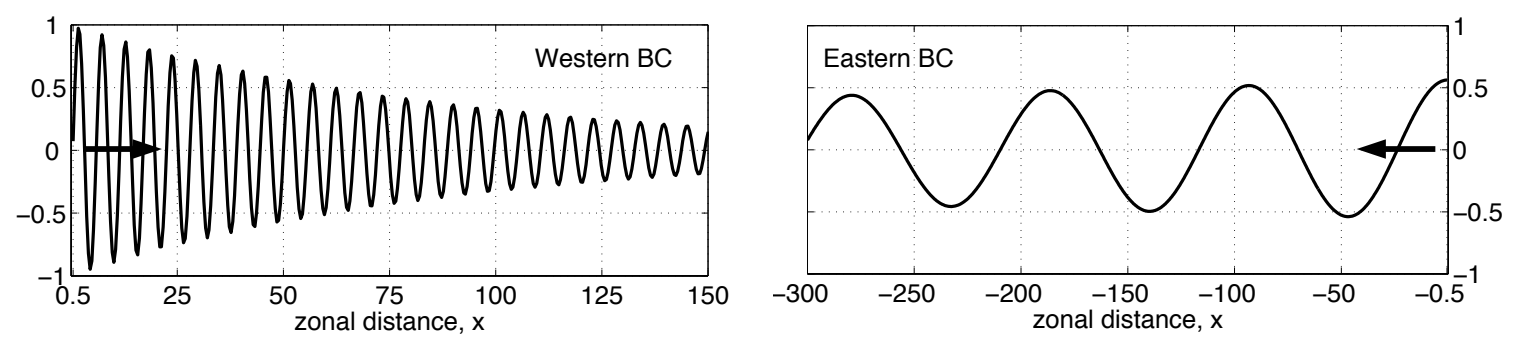

\begin{tabular}{|l||l|l|l|l|}
\hline & $m$ & $c_{r}$ & $m c_{i}$ & $k$ \\
\hline Western BC & 0.348 & 1.153 & $3.2 \times 10^{-3}$ & $-1.140+i 0.011$ \\
\hline Eastern BC & 0.348 & 0.773 & $3.2 \times 10^{-3}$ & $-0.068-i 0.001$ \\
\hline
\end{tabular}

Figure 2-4: Structure of a radiating wave for the western and eastern boundary current for the barotropic case. Only the real part of the solution in the far field $\phi(x)$ is plotted as a function of $x$.

infinity while the growth rate decreases, which indicates that in the limit of zero growth rate the solution is a pure wave $\left(k_{i}=0\right)$. The modes have a radiating wave structure, defined by $\left|k_{r}\right|>\left|k_{i}\right|$ or positive values for $\log \left(\left|k_{r}\right| /\left|k_{i}\right|\right)$, over some interval of meridional wavenumbers before they stabilize. The structure of the eigenmodes in the far field depends strongly on the meridional wavenumber and the growth rate. In general, the weaker the growth rate, the shorter in the zonal direction are the radiated waves and the larger the amplitude envelope decay scale.

In Fig. 2-4 a typical example of a far field solution is shown. The radiating wave has a meridional wavelength of $2 \pi / m \approx 18$ current widths, zonal wavelength of $2 \pi / k_{r} \approx 5$ current widths and envelope decay scale $1 / k_{i} \approx 90$ current widths. For example, if the parameter $\beta=0.5$ is representative of a $100 \mathrm{~km}$ wide current with speed $40 \mathrm{cms}^{-1}$, then the radiated wave has zonal wavelength of $500 \mathrm{~km}$, envelope decay scale of $9000 \mathrm{~km}$ and a growth rate approximatively $(2.5 y e a r s)^{-1}$.

\section{Eastern boundary current}

In order to satisfy the condition of a vanishing perturbation at infinity, a western boundary current selects solutions in the far field that have positive zonal group velocity while for an eastern boundary current, the solutions have negative zonal 
group velocity. This difference has a strong effect on the stability properties of the current.

The short wave end of the explored range of meridional wavenumbers is similar for the western and eastern configurations. There is a single unstable eigenvalue (dot-dashed black line in Fig. 2-2) that asymptotes to $c=0.5+i 0.5$ when $m \rightarrow$ $+\infty$. Looking back at equation (2.3), one can see that in this limit the term $\beta /$ im responsible for the asymmetries in the propagation properties between east and west is not important. When the meridional wavenumber is decreased however, differences appear. The meridional phase speed $c_{r}$ of the mode decreases, unlike for the western boundary current case. When the mode finally stabilizes at the critical wavenumber $m_{*}=0.080$, its meridional phase speed is equal to minus one, i.e it is opposite to the basic state current.

In addition to this mode, there are also other unstable eigenvalues (a representative is shown in Fig. 2-2 with a dot-dashed gray line). Again, due to the trend of $c_{i}$ to decrease to zero while $c_{r}$ goes to 1 , when the meridional wavenumber is increased, it is thought that these eigenvalues originate in their short wave limit from the singular point $c=1$ but because of the singularity, the limit cannot be reached numerically. The meridional phase speed for these modes decreases when $m$ gets smaller and becomes $c_{r}=-1$, i.e. opposite to the basic state current, when the modes stabilize (see Fig. 2-2a). Their growth rates are zero in both extremes and reach a maximum somewhere in between (see Fig. 2-2b). There are infinitely many eigenvalues (not only the one shown on the figures) with similar behavior that reach their maximum growth rate at smaller and smaller values of $m$. A major difference from the western boundary current is that the accumulation point for these eigenvalues is $m=0$ rather than $m$ finite. In other words, there is no long meridional wave cut-off for the linear stability of an eastern boundary current.

Concerning the radiating nature of the instabilities, the logarithm of the ratio $\left|k_{r}\right| /\left|k_{i}\right|$, where $k$ is the zonal wavenumber in the far field, is plotted in Fig. 2-3b as a function of the meridional wavenumber. Since for all eastern boundary current modes 
the meridional phase speed changes sign (it goes from being positive to -1 when $m$ is decreased, see Fig. 2-2a), so does the real part of the zonal wavenumber in the far field. This corresponds to the minima of the dashed curves in Fig. 2-3b, where the far field solution is characterized with $k_{r} \approx 0$. The solution has a radiating wave structure, as indicated by the positive values for $\log \left(\left|k_{r}\right| /\left|k_{i}\right|\right)$, to the left of the minimum (for all modes) and to the right of the minimum (for all but the leading unstable mode).

The long meridional wave end corresponds to a radiating instability, as for the western boundary current, since both $k_{i}$ and the growth rate vanish. However, in this limit $k_{r} \approx 10^{-4}$ or smaller depending on the mode, which leads to radiating waves with extremely long zonal wavelengths on the order of ten thousand current widths or more. Unlike for the western boundary current, there is an infinite number of eigenmodes with radiating wave structure toward the short meridional wave end. For all modes but the most unstable one, for values of the meridional wavenumber to the right of the minimum, the far field solution is characterized by $\left|k_{r}\right| \gg\left|k_{i}\right|$ (positive values for $\log \left(\left|k_{r}\right| /\left|k_{i}\right|\right)$ ) while the growth rate is very weak, which is an indication of an eigenmode with horizontally radiating structure. In general, the smaller the meridional wavenumber, the stronger the growth rate and the greater the zonal wavelength of the radiated wave with typical values between 20-2000 current widths.

An example of a far field solution on the short meridional wave side of the minimum is shown on Fig. 2-4. The radiated wave has a meridional wavelength of $2 \pi / m \approx 18$ current widths, zonal wavelength of $2 \pi / k_{r} \approx 90$ current widths and envelope decay scale $1 / k_{i} \approx 1000$ current widths. The solution has been chosen to have exactly the same growth rate as the solution for the western boundary discussed before. For the same growth rate, its longer envelope decay scale is due to the greater zonal group velocity: $c_{g}^{x}=-3.69$ for the eastern compared to $c_{g}^{x}=0.29$ for the western boundary current, where the group velocity $c_{g}^{x}$ is given in units of the current velocity $V_{*}$. This is consistent with the analysis in Section 2.2.2 that the radiated waves from the eastern side are characterized with longer zonal wavelengths and a 
slower amplitude envelop decay due to the greater zonal group velocities than their western boundary counterpart.

As a final remark, in this barotropic model the only energy source for the growing instabilities is associated with the jump in the basic state velocity. Thus, the radiating waves are considered the result of a Kelvin-Helmholtz type instability of the flow.

\subsection{The baroclinic case}

In this section the problem of the linear stability of a purely baroclinic meridional current adjacent to a motionless far field is examined using a 2-layer QG model. The introduction of vertical structure leads to a model able to represent more realistic processes. Specifically, the mean flow instabilities can be either of the Kelvin-Helmholtz type, as in the barotropic case presented in Section 2.2, or baroclinic instabilities because of the presence of vertical shear.

\subsubsection{Formulation}

For the 2-layer case, the basic state profile is again piecewise constant as sketched in Fig. 2-1, except that now the flow is chosen to be purely baroclinic

$$
V_{1,2}= \begin{cases} \pm \frac{V_{S}}{2} & ,|x|<x_{0} \\ 0 & ,|x|>x_{0}\end{cases}
$$

Without loss of generality, the vertical shear $V_{S}$ is chosen to be positive. The perturbation streamfunctions for each layer $\psi_{n}(x, y, t)$ are once more decomposed into normal modes, $\psi_{n}(x, y, t)=\operatorname{Re}\left\{\phi_{n}(x) e^{i m(y-c t)}\right\}$, where the amplitudes $\phi_{n}(x)$ satisfy the linearized quasi-geostrophic potential vorticity equation

$$
\left(V_{n}-c\right)\left[\phi_{n}^{\prime \prime}-m^{2} \phi_{n}+(-1)^{n} F_{n}\left(\phi_{1}-\phi_{2}\right)\right]+\frac{\bar{Q}_{n, y}}{i m} \phi_{n}^{\prime}-\bar{Q}_{n, x} \phi_{n}=0
$$


Here, $\left\{\bar{Q}_{n, x}, \bar{Q}_{n, y}\right\}$ is the potential vorticity gradient of the basic state given by

$$
\bar{Q}_{n, x}=\frac{d^{2} V_{n}}{d x^{2}}+(-1)^{n} F_{n}\left(V_{1}-V_{2}\right), \quad \bar{Q}_{n, y}=\beta .
$$

All variables above are non-dimensionalized using as scales the vertical shear $V_{S}$ and the Rossby deformation radius $L_{d}=\sqrt{2 g^{\prime} H_{1} H_{2} / f_{0}^{2}\left(H_{1}+H_{2}\right)}$. The nondimensional parameters that appear in equations (2.12) and (2.13) are the scaled planetary vorticity gradient $\beta=\beta_{0} L_{d}^{2} / V_{S}$ and the parameters $F_{n}$ which are function of the layer depths, $F_{n}=2 H_{1} H_{2} / H_{n}\left(H_{1}+H_{2}\right)$ with $F=F_{1}+F_{2}=2$. Similar to the barotropic case, the jump conditions (2.5), as well as the no-normal flow condition on the solid wall are applied to each layer. The method of finding the eigenvalues is essentially the same except for a larger problem size. More details on the method of solution are given in Appendix A.2.

The analysis from the barotropic case regarding how to identify the radiating instabilities is helpful for the 2-layer model as well, although the situation is a little more complex. In the 2-layer model, for given choice of parameters $\beta, F_{1} / F_{2}$ and meridional wavenumber $m$, the solution in the far field is a superposition of two waves with complex zonal wavenumbers $k_{b t}$ and $k_{b c}$, related to the frequency $\omega=\mathrm{cm}$ by the barotropic and baroclinic Rossby wave dispersion relations, respectively

$$
c m=-\frac{\beta k_{b t}}{k_{b t}^{2}+m^{2}}, \quad c m=-\frac{\beta k_{b c}}{k_{b c}^{2}+m^{2}+F} .
$$

For both the barotropic and baroclinic part of the far field solution, an analysis similar to that in Section 2.2 .2 can be made. In particular, for an eigenvalue $c$ satisfying the problem, there are two possible values for each of the wavenumbers $k_{b t}$ and $k_{b c}$ that have opposite signed imaginary parts and zonal group velocities. The solution for a western boundary current has positive zonal group velocity while for an eastern boundary current it has negative zonal group velocity, so that in both cases we have a vanishing perturbation at infinity. A solution qualifies as a radiating wave if in the limit of becoming neutrally stable, the imaginary part of $k_{b t}$ or of both $k_{b t}$ 
and $k_{b c}$ go to zero. The physical explanation behind this is the following. Since the phase speed range of barotropic Rossby waves $\left(\left|c_{r}\right|<\beta / 2 m^{2}\right)$ is wider than that of baroclinic Rossby waves $\left(\left|c_{r}\right|<\beta / 2 m \sqrt{m^{2}+F}\right)$, it may happen so that a solution has a radiating barotropic part but non-radiating baroclinic part. If however, the phase speed $c$ lies within the range of the free baroclinic Rossby waves, then we have a solution that is a radiating wave and could have both barotropic and baroclinic components.

\subsubsection{Energetics}

In the 2-layer QG model the energy for the growing instabilities, be they radiating or not, can come from two sources - Kelvin-Helmholtz type instability or baroclinic instability. In order to determine in what proportions these two sources contribute, one needs to consider the energy balance.

The energy equation can be derived by multiplying equation (2.12) by the complex conjugate amplitude $\phi_{n}^{*}$ weighted by the layer depth $d_{n}=H_{n} / H$ and summing over the two layers. After several manipulations and using the fact that $d V_{n} / d x$ is zero for the piecewise constant velocity profile used here, one can write the final result as

$$
2 m c_{i} E=m F_{0}\left(V_{1}-V_{2}\right) \operatorname{Im}\left\{\phi_{1} \phi_{2}^{*}\right\}+\frac{d S}{d x},
$$

where $E=\frac{F_{0}}{2}\left|\phi_{1}-\phi_{2}\right|^{2}+\sum_{n=1}^{2} \frac{d_{n}}{2}\left(\left|\phi_{n}^{\prime}\right|^{2}+m^{2}\left|\phi_{n}\right|^{2}\right)$ is the total (potential plus kinetic) wave energy of the system with $F_{0}=d_{1} F_{1}=d_{2} F_{2}$. The quantity $S$ is a flux term defined as

$$
S=\sum_{n=1}^{2} \frac{\beta}{2} d_{n}\left|\phi_{n}\right|^{2}-d_{n} \operatorname{Im}\left\{m\left(V_{n}-c\right) \phi_{n}^{*} \frac{d \phi_{n}}{d x}\right\} .
$$

The energy flux $S$ is zero at the solid wall and at infinity and undergoes a jump, proportional to the jump in the basic state velocity, at the point where the velocity profile is discontinuous. Integrating equation (2.15) over the whole domain - from the wall to infinity for a western boundary current or from minus infinity to the wall 
for an eastern boundary current - leads to the following energy balance

$$
0<\int 2 m c_{i} E d x=\underbrace{\int_{-x_{0}}^{x_{0}} m F_{0}\left(V_{1}-V_{2}\right) \operatorname{Im}\left\{\phi_{1} \phi_{2}^{*}\right\} d x}_{B C}+\underbrace{\Delta[S]}_{B T}
$$

For a linearly unstable, growing mode, the terms on the right-hand side have to sum to a positive number. Term $B C$ is the contribution from baroclinic instability where perturbations grow feeding on the potential energy of the basic state flow, proportional to the vertical shear $\left(V_{1}-V_{2}\right)$. Term $B T$ is the contribution from the flux term which for $c_{i} \neq 0$ is non-zero only because there is a jump in the basic state velocity profile at $x=+x_{0}$ for a western boundary current or at $x=-x_{0}$ for an eastern boundary current. This is interpreted as a Kelvin-Helmholtz type of instability that arises in the presence of discontinuous velocity profiles. In the barotropic model, the only source for growing perturbations is term BT, while in the 2-layer model terms $B C$ and $B T$ can combine in different ways and lead to growth.

\subsubsection{Results}

The baroclinic problem is characterized by three non-dimensional parameters which are $\beta, F_{1} / F_{2}$ or the ratio of the layer depths, and the non-dimensional width of the current $2 x_{0} / L_{d}$. In this section, results from calculations made with specific values of these parameters are shown. The layer depths are taken to be equal which translates into $F_{1}=F_{2}=1$, the width of the current is set to 10 deformation radii and $\beta=\beta_{0} L_{d}^{2} / V_{S}=0.5$. As before, when solving the stability problem, the main objectives are to find the unstable eigenvalues, follow them as a function of the meridional wavenumber $m$ and determine whether they are radiating.

Before going into more details about the results, some general observations can be made that hold for both the western and eastern boundary current configurations. An examination of the problem solution shows that the unstable eigenvalues, if there are such, have real parts situated between $-0.5<c_{r}<0.5$, the non-dimensional 
lower and upper layer basic state velocity. In other words, the semi-circle theorem seems to apply although it can not be proved for the meridional case (Walker and Pedlosky, 2002). Furthermore, with the equal layer depth assumption, the stability problem has the following symmetry property. If $c=c_{r}+i c_{i}$ is an eigenvalue of the problem, with corresponding eigenvectors $\left\{\phi_{1}(x), \phi_{2}(x)\right\}$, then $\tilde{c}=-c_{r}+i c_{i}$ is also an eigenvalue, with corresponding eigenvectors $\left\{\phi_{2}^{*}(x), \phi_{1}^{*}(x)\right\}$. Thus, there are two possibilities for the unstable eigenmodes: either they have a non-zero real phase speed, in which case they come in pairs $c= \pm c_{r}+i c_{i}$, or they have a zero real phase speed $c=0+i c_{i}$. The last ones are not of interest for radiating instabilities since $c_{r}=0$ implies $\operatorname{Re}\left\{k_{b t}, k_{b c}\right\}=0$, i.e no waves in the far field.

\section{Western boundary current}

In the short wave end of the explored range of meridional wavenumbers, there is a single pair of unstable eigenvalues that asymptotes to $c= \pm 0.25+i 0.25$ as $m \rightarrow+\infty$ (black solid line in Fig. 2-5a, b). Again, as in the barotropic case, the lack of short wave cut-off is related to the choice of piecewise constant basic state profile with infinitely thin region of horizontal shear.

In addition to the leading pair, there are other pairs of unstable eigenvalues (two representatives are shown in Fig. 2-5a, b with gray solid lines). They originate from eigenvalues with zero real part (gray dashed lines in Fig. 2-5a, b) that collide and split into two unstable eigenvalues with non-zero real parts. When the meridional wavenumber is decreased, for all unstable pairs, the meridional phase speed $c_{r}$ goes to \pm 0.5 , the upper and lower layer velocities, while the growth rate decreases. It was not possible to reach exactly the zero growth rate limit since the points $c= \pm 0.5$ are singular and it is very difficult to track eigenvalues in their vicinity. It is thought however that the modes stabilize when their meridional phase speed reaches the upper or lower layer velocity because of the decreasing trend for $c_{i}$. There is an infinite number of unstable pairs that originate from zero meridional phase speed modes at smaller and smaller meridional wavenumbers. Their accumulation point is however 

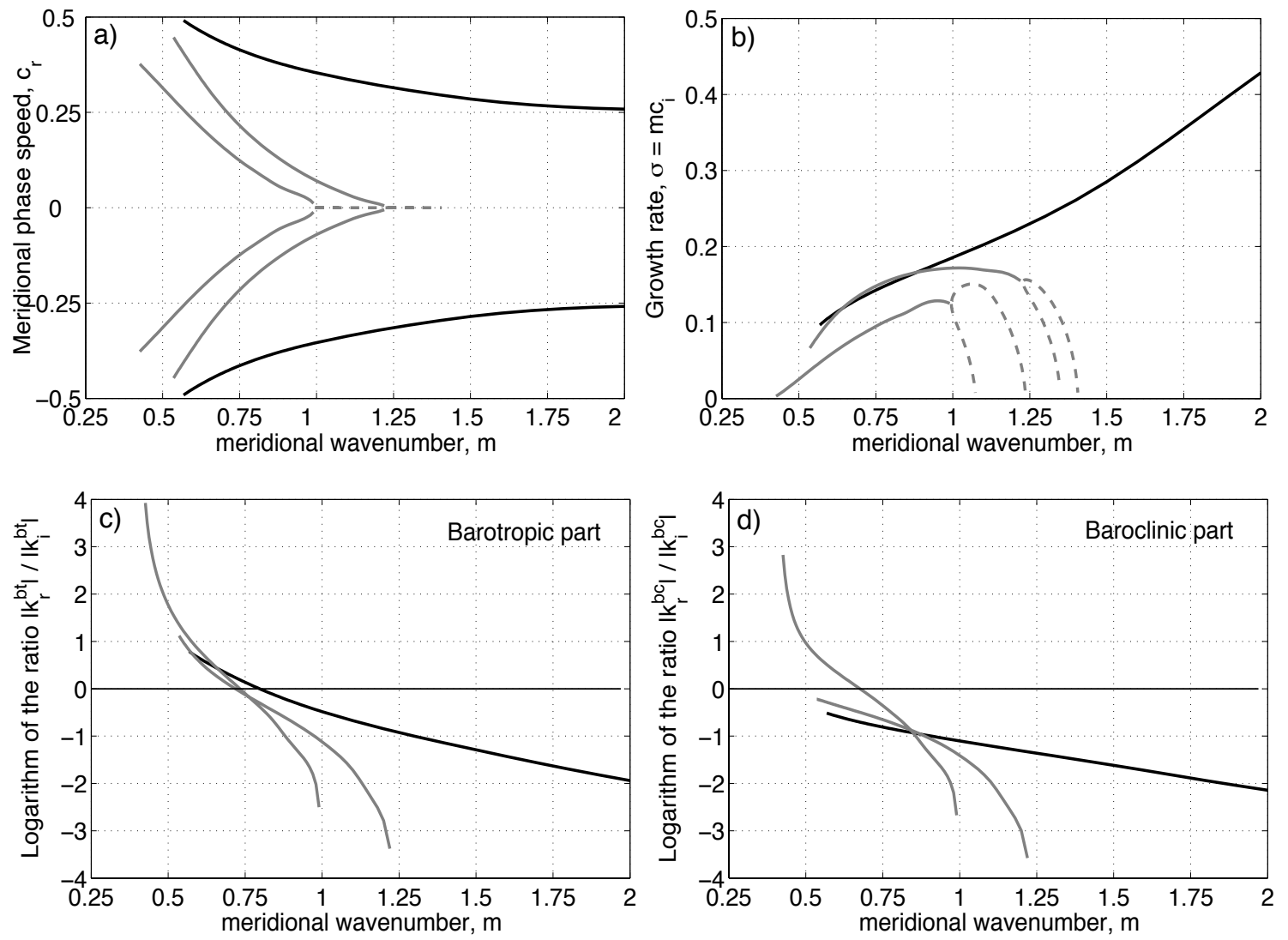

Figure 2-5: For the baroclinic western boundary current configuration with $\beta=0.5$ and $F_{1}=F_{2}=1$, meridional phase speed (a), growth rate (b) and logarithm of the ratio $\left|k_{r}\right| /\left|k_{i}\right|$ for the barotropic (c) and baroclinic (d) part of the far field solution as a function of the meridional wavenumber. In each panel the first 10 unstable eigenvalues are shown using black solid lines for the leading unstable pair, gray solid lines - next unstable pairs, gray dashed lines - eigenvalues with $c_{r}=0$ (non-radiating).

some finite critical wavenumber below which there are no more unstable modes. Thus, similarly to the barotropic western boundary current, there is a long meridional wave cut-off for the linear stability of a purely baroclinic western boundary current.

Concerning the radiating nature of the instabilities, the logarithm of the ratios $\left|k_{r}^{b t}\right| /\left|k_{i}^{b t}\right|$ and $\left|k_{r}^{b c}\right| /\left|k_{i}^{b c}\right|$, where $k^{b t}$ and $k^{b c}$ are the zonal wavenumbers for the barotropic and baroclinic part of the far field solution, are plotted as a function of the meridional wavenumber in Fig. 2-5c, d respectively. These plots show only the modes with non-zero meridional phase speed which are the only ones that can possibly have 
wave structure in the far field. Although it was not possible to reach exactly the limit $c_{i}=0$, there is an indication that for for both the barotropic and the baroclinic part of the solution the long wave end of the explored range of meridional wavenumbers is radiating since $\left|k_{r}\right| \gg\left|k_{i}\right|$ while $m c_{i} \rightarrow 0$. This is especially true for the pairs of modes that destabilize at smaller meridional wavenumbers and not so much for the leading pair of unstable modes. Note that, although the eigenmodes with radiating structure in the far field are found toward the long wave end of the explored range of meridional wavenumbers $(m<0.75)$, the corresponding meridional wavelength of the disturbances is actually not so large - it is only a couple of deformation radii.

Finally, it is worth noticing that the stability picture, where pairs of unstable modes originate from modes with zero meridional phase speed and stabilize when they reach the basic state velocities, is very similar to what is occurring in a meridional flow confined in a channel, the configuration studied in detail in Walker and Pedlosky (2002) and Pedlosky (2002). The reason for the instability in this case is identified as being the destabilization of Rossby normal modes by the vertical shear. The resemblance to the channel case suggests that despite the addition of a motionless far field on one side of the meridional flow, the same physical mechanism for the instability may be in play.

\section{Eastern boundary current}

The eigenvalue analysis of an eastern boundary current is qualitatively similar for the most part to the western counterpart. In the short wave end of the explored range of meridional wavenumbers, there is a single unstable pair that asymptotes to $c= \pm 0.25+i 0.25$ (black solid line in Fig. 2-6a,b). Additional pairs of unstable eigenvalues appear from splitting of zero meridional phase speed eigenvalues (two representatives are shown in Fig. 2-6a, b with gray solid line). When the wavenumber is decreased, the meridional phase speed for all unstable pairs goes toward $c_{r}= \pm 0.5$, the upper and lower basic state velocities, where the modes are thought to stabilize although the exact zero growth rate limit cannot be reached computationally. This 

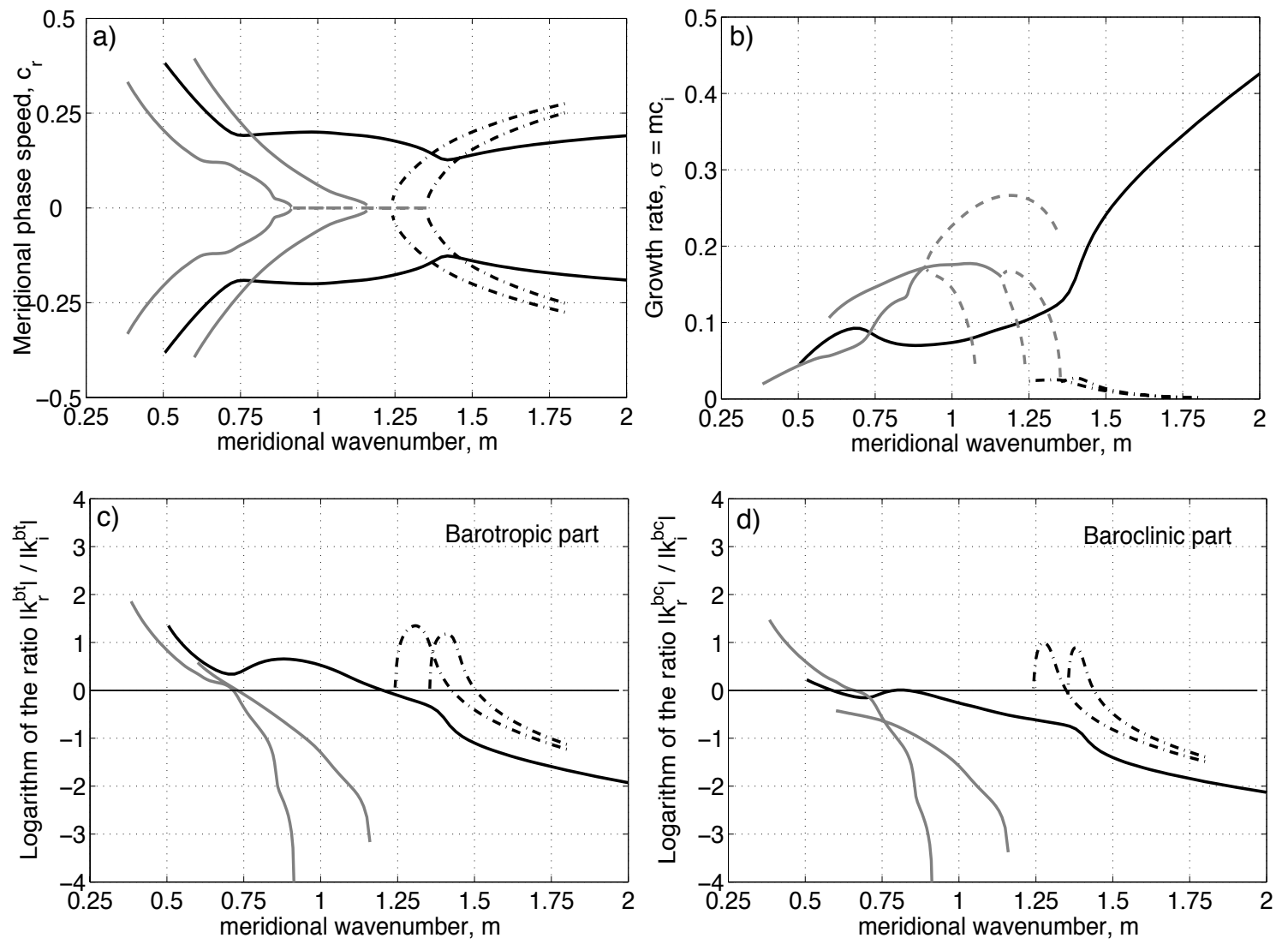

Figure 2-6: For the baroclinic eastern boundary current configuration with $\beta=0.5$ and $F_{1}=F_{2}=1$, meridional phase speed (a), growth rate (b) and logarithm of the ratio $\left|k_{r}\right| /\left|k_{i}\right|$ for the barotropic (c) and baroclinic (d) part of the far field solution as a function of the meridional wavenumber. In each panel the first 14 unstable eigenvalues are shown using black solid lines for the leading unstable pair, gray solid lines - next unstable pairs, gray dashed lines - eigenvalues with $c_{r}=0$ (non-radiating) and black dot-dashed lines - weakly unstable pairs, present in the eastern case only.

again bears similarities to the instability of a meridional channel flow studied in Walker and Pedlosky (2002).

There are also some differences from the western case. First of all, there is a range of meridional wavenumbers over which the additional pairs are the most unstable modes with growth rates almost as twice as large as the leading pair. Another difference is that there is a whole group of weakly unstable eigenmodes, not present in the western case (two such pairs, the most unstable ones, are shown in Fig. 2-6a, b with a black dot-dashed lines). These weakly unstable modes are characterized with merid- 
ional phase speeds that decrease from $c_{r}= \pm 0.5$ toward $c_{r}=0$ when the meridional wavenumber is decreased. These modes seem to be at the origin of the zero meridional phase speed modes (gray dashed line in Fig. 2-6a,b) - when a pair of weakly unstable modes reaches $c_{r}=0$, they collide and a single unstable eigenvalue with $c_{r}=0$ appears. As we will see later, the energetics for these weakly unstable modes is also different, which suggests that a different mechanism for the instability is at play. Finally, similar to the barotropic case, the accumulation point for the infinite number of unstable modes is $m=0$ so that there is no long meridional wave cut-off for the linear stability of a purely baroclinic eastern boundary current.

Concerning the presence of radiating waves, the logarithm of the ratios $\left|k_{r}^{b t}\right| /\left|k_{i}^{b t}\right|$ and $\left|k_{r}^{b c}\right| /\left|k_{i}^{b c}\right|$ are plotted for all non-zero meridional phase speed modes in Fig. 2-6c, d respectively. In a comparable way to the western case, it is the long wave end of the explored range of meridional wavenumbers that seems to be radiating since $\left|k_{r}\right| \gg\left|k_{i}\right|$ while $m c_{i} \rightarrow 0$ for both the barotropic and the baroclinic part. Exceptions are the weak growth rate eigenmodes that exist in the eastern case only. For these modes, neither their short or their long wave limit is radiating even though the eigenvectors have a radiating wave structure in the far field (positive values for $\log \left(\left|k_{r}^{b t}\right| /\left|k_{i}^{b t}\right|\right)$ and $\left.\log \left(\left|k_{r}^{b c}\right| /\left|k_{i}^{b c}\right|\right)\right)$ for some range of meridional wavenumbers in between.

\section{Radiating solutions}

Example of radiating wave solutions for the western and eastern configurations are shown in Fig. 2-7.

First of all as could be expected, waves from the western side are characterized with smaller zonal wavelengths and faster amplitude decay away from the current compared to the eastern case. For the western boundary current solution, the radiated barotropic and baroclinic waves have comparable zonal wavelengths on the order of 2-3 deformation radii and an envelope decay scale on the order of 1-2 deformation radii. For the eastern boundary current solution, the baroclinic wave is of zonal length $2 \pi / k_{r}^{b c} \approx 10$ deformation radii while the barotropic wave is much longer, 

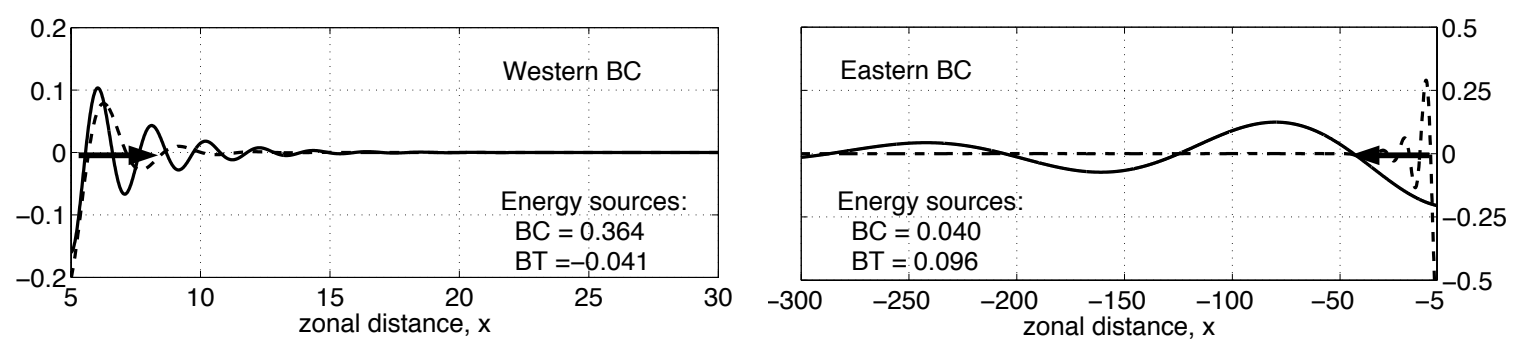

\begin{tabular}{|l||l|l|l|l|l|}
\hline & $m$ & $c_{r}$ & $m c_{i}$ & $k_{b t}$ & $k_{b c}$ \\
\hline Western BC & 0.486 & -0.326 & $2.1 \times 10^{-2}$ & $3.021+i 0.419$ & $2.168+i 0.715$ \\
\hline Eastern BC & 0.390 & -0.324 & $2.1 \times 10^{-2}$ & $0.039-i 0.007$ & $0.630-i 0.154$ \\
\hline
\end{tabular}

Figure 2-7: Structure of a radiating wave for the western and eastern boundary current for the baroclinic case. Only the real part of the solution in the far field $\phi(x)$ is plotted as a function of $x$. Solid line - barotropic part, dashed line - baroclinic part of the solution.

$2 \pi / k_{r}^{b t} \approx 160$ deformation radii. Since the envelope decay scale for the baroclinic part is much shorter compared to that for the barotropic part however (6 compared to 140 deformation radii), the solution far away from the current is predominantly barotropic.

Second, a peculiarity about the horizontal structure of the far field solution is brought to light if one looks at the meridional wavelength of the radiated waves. For both the eastern and the western case solutions, the meridional wavelength of the waves is on the order of $2 \pi / m \approx 15$ deformation radii while their zonal wavelengths are significantly different. We find that waves radiated from the eastern side tend to be asymmetric, in the sense that they are much longer in the zonal direction than in the meridional. This leads to velocity field with zonal component much larger than the meridional component which would make the radiating waves appear, as they propagate in the far field, more like zonal jets than localized wave packets or eddies.

\section{Energetics}

An inspection of the energy balance for the unstable eigenmodes can give some insight into the processes responsible for the instability. 
For the leading pair (black solid lines in Fig. 2-5 and 2-6), especially in the short wave end of the explored range of meridional wavenumbers, the most important energy source is term $B T$ or Kelvin-Helmholtz type instability related to the jump in the basic state velocity profile. This holds for both the western and eastern boundary current setups and supports the idea that the lack of short wave cut-off is due to the choice of discontinuous velocity profile.

Concerning the other pairs of unstable eigenmodes (solid gray lines in Fig. 2-5 and 2-6), there is a significant difference between the western and eastern configurations. For the western case, the dominant energy source is term $B C$ or the baroclinic instability while term $B T$, related to the jump in the basic state velocity, is negligible (see the western solution in Fig. 2-7). For the eastern case, both terms $B C$ and $B T$ are positive and contribute in comparable amounts (see the eastern solution in Fig. 2-7). The fact that in both configurations, the baroclinic conversion term $B C$ is important for the pairs of eigenmodes originating from splitting of modes with zero real part eigenvalues further supports the connection to the meridional channel flow instability due to the destabilization of Rossby normal modes by the vertical shear, as discussed in Walker and Pedlosky (2002) and Pedlosky (2002).

Finally, for the weak growth rates eigenmodes that exist only in the eastern configuration (black dot-dashed lines in Fig. 2-6), the baroclinic conversion term BT is negative, while term $B C$ related to the jump in the mean velocity is positive and slightly bigger in magnitude, so that we have a growth overall. Thus, these modes are the result of a baroclinic type of instability and are different from all the other radiating modes which are generated by a mixed barotropic-baroclinic instability of the basic state flow.

One can use also the energy balance to get some indications about the potential effect of the radiating modes on the current. The solutions shown in Fig. 2-7 have been plotted with mode amplitude chosen so that the perturbation velocities within the boundary current region be of the same order as the basic state current itself. Although one would not expect a linear stability analysis to hold at such large ampli- 
tudes, this is a reasonable assumption for regions of unstable oceanic currents where the meanders lead to perturbations of the same order as the mean, and is done in order to get realistic magnitude for the energy fluxes. Given the total energy contained in the basic state $\bar{E}=\int_{-x_{0}}^{x_{0}}\left(V_{1}^{2}+V_{2}^{2}+V_{S}^{2} x^{2}\right) / 2 d x$, one can use then the fluxes BC and BT to find the time needed to utilize all of the basic state energy toward growing perturbations. Note that in the framework of the linear stability analysis performed here, the flow is not actually evolving in time. The basic state velocity profile is constantly supplied with energy from some external forcing (wind for example) so that it is fixed in time. The depletion time scale defined above is thus only a hypothetical quantity helpful in judging the effect of the radiating modes on the current while no actual time evolution computations are performed.

The depletion time scales found using the fluxes for the specific solutions in Fig. 27 are on the order of 50-70 time units. Those are comparable to the growth time scale which is $1 / m c_{i} \approx 48$ time units. If the non-dimensional parameter $\beta=0.5$ is representative of a current with deformation radius $L_{d}=60 \mathrm{~km}$ and vertical shear $V_{S}=15 \mathrm{~cm} / \mathrm{s}$, then the depletion times are on the annual scale which implies a minor effect on the current.

\subsection{Discussion and conclusions}

In this paper we have performed a linear stability analysis of a meridional boundary current adjacent to a motionless far field. The current is idealized as a piecewise constant linear profile as in Fantini and Tung (1987), which allows the stability problem to be reduced to a non-linear algebraic equation that can be solved numerically. We are interested in a special type of instability of this system. When the phase speed and wavenumber of the disturbances within the unstable region are such that they match those of the freely propagating Rossby waves in the far field, temporally growing radiating waves with amplitude envelopes that decay slowly with distance from the source may appear. These are called radiating instabilities. The existence 
of radiating instabilities is of interest because, even if the radiating modes are not the most unstable modes, they are the only ones that reach the neutral far field. By transporting perturbation energy away, they have the ability to affect the circulation far from the locally unstable region where the perturbations are generated.

We have considered two different cases of a basic state flow: a purely barotropic and a purely baroclinic meridional velocity profile since it was determined that the stability of a more general flow, that is still piecewise constant but has both barotropic and baroclinic components, is a mix of the behavior of the purely barotropic and purely baroclinic cases.

The first major conclusion of this paper is that unlike zonal currents, for which special circumstances are needed such as baroclinic or westward component of the basic state flow (Talley, 1983a,b), unstable meridional currents are generally characterized by eigenmodes that have horizontally radiating structure. The radiating modes are not necessarily the most unstable ones but there are usually several of them for a given set of parameters. In the 2-layer case, the radiating solutions have both barotropic and baroclinic components.

A second major conclusion of this paper concerns the differences in the stability properties of western and eastern meridional boundary currents. For instance, it was found that western boundary currents are linearly stable to perturbations with meridional wavenumbers below some critical value while there is no such long meridional wave cut-off for the linear stability of eastern boundary currents. What is at the base of these differences is the requirement that the zonal group velocity of the radiated waves be away from the locally unstable region. Consequently, western boundary currents radiate short Rossby waves $\left(k_{r}>m\right)$, that have small eastward group velocity and rapidly decaying amplitude envelope away from the current. Eastern boundary currents on the other hand, radiate long Rossby waves $\left(k_{r}<m\right)$, that have large westward group velocity and slowly decaying amplitude envelope away from the current. It was determined that not only do radiating waves from the eastern side penetrate further into the far field region, but there is a greater number of them and they can 
be found over a wider range of meridional wavenumbers.

Another peculiarity of the eastern boundary current radiating waves is that they tend to have an asymmetrical horizontal structure with zonal wavelength several times larger than the meridional wavelength. This leads to a velocity field with zonal component much larger than the meridional component which would make appear the radiating waves, as they propagate in the far field, more like zonal jets than localized wave packets or eddies. Circulation in the form of multiple zonal jets has been observed in the real ocean (Maximenko et al., 2005). In particular, the eastern parts of all basins at mid-latitudes contain signatures of quasi-steady zonal striations with meridional scale on the order of $200-300 \mathrm{~km}$ and extending zonally for thousands of kilometers (Maximenko and Niiler, 2006). The origin of these jets is not yet fully understood. The present study suggests the possibility that the observed zonal jets may be related to radiating instabilities of eastern boundary currents.

We have looked also at the energy balance which gives some insight into the sources for the instabilities. In the barotropic model, the only energy source is a Kelvin-Helmholtz type of instability due to the discontinuous velocity profile. In the 2-layer case, a second possible energy source is baroclinic instability because of the presence of vertical shear. There are some differences between the energy balance for the western and eastern case. However, the fact that the baroclinic conversion term contributes significantly to the energy balance for all unstable modes except the leading one, for both eastern and western boundary currents, suggests a connection to the meridional channel flow instability studied in Walker and Pedlosky (2002).

As a final word, although the model used in this study is very idealized, it leads to some interesting conclusions concerning the differences between eastern and western meridional boundary currents and the characteristics of the radiating waves which are worth pursuing using more realistic models. 


\section{Chapter 3}

\section{Part 2: A two-layer QG model for a thermally-forced ocean}

\subsection{Introduction}

The ocean circulation is forced at the surface by both wind stress and large scale buoyancy fluxes resulting from heat and freshwater exchange with the atmosphere. The direct effects of the wind and buoyancy forcing are strongest down to a depth of roughly $1 \mathrm{~km}$ leading to a circulation that is vigorous in the upper ocean and much more sluggish in the abyssal ocean. What makes the problem of determining the ocean circulation a complex one, is its intrinsic nonlinearity. It is advective dynamics that determine to a large extent the horizontal and vertical structure of the density field which in turn, through the effects of buoyancy and rotation, shapes the motion field (Pedlosky, 1998).

Quasi-geostrophic (QG) layer models with only wind forcing have been used as a simple framework to study the mid-latitude ocean circulation. In these models, the mean vertical stratification is prescribed by specifying a certain number of isopycnal layers. Within each layer the motion is vertically uniform and quasi-geostrophic with the vertical excursions of the density interfaces constrained to be small compared to the layer depth. Commonly, the circulation in QG models is assumed adiabatic 
so that the amount of water contained between any two isopycnal surfaces remains unchanged with time. The adiabatic assumption signifies also that in absence of dissipation, the potential vorticity of the fluid parcels in the deep layers not directly exposed to the wind stress is conserved, which poses a substantial constraint on the fluid motion.

Despite their simplicity, barotropic and multi-layer QG models forced by a simple sinusoidal Ekman pumping velocity are able to reproduce several aspects of the complex temporal and spatial behavior of the subtropical/subpolar gyre system and the free mid-latitude jet. Consequently, QG models have been proven very useful in isolating and understanding several basic processes that are active in the real ocean. Some examples of such processes are eddy-driven flows (Holland, 1978; Holland and Rhines, 1980), western boundary layer and recirculation dynamics (Cessi et al., 1987; Lozier and Riser, 1989; Berloff and McWilliams, 1999b), free jet dynamics (Jayne and Hogg, 1999), the role of eddies and friction in controlling the mean circulation (Fox-Kemper, 2003), and numerous others. There is also an extensive literature on the internal variability of the wind-driven circulation based on QG models. Internal variability is defined as the variability that results under steady forcing conditions due to the nonlinearity of the dynamics. Because of their simplicity and symmetry properties, QG models have been extremely convenient for identifying some basic mechanisms that can then be traced back to more complex models, and eventually to the real ocean. Phenomena such as multiple steady states (Dijkstra and Katsman, 1997; Simonnet, 2005), transition to complex time-dependent behavior (Primeau, 1998; Simonnet and Dijkstra, 2002), and different mechanisms for low-frequency variability (McCalpin and Haidvogel, 1996; Berloff and McWilliams, 1999a; Ghil et al., 2002; Nauw et al., 2004) are among the many topics explored.

QG models of different complexity have been used in these studies - from barotropic, to reduced gravity, to multi-layer models, but as already mentioned, in all of these studies the assumption is usually made that the ocean dynamics are adiabatic, i.e. that there is no exchange of water between the different density layers. In reality 
however, the ocean is subject to surface buoyancy fluxes that modify the density of the water column affecting thus the horizontal and vertical structure of the density field which in turn alters the velocity field. Another consequence of the presence of cross-isopycnal fluxes is that it breaks down the potential vorticity conservation, a property on which wind-driven theories rely heavily. For a wind-only driven adiabatic ocean where potential vorticity is conserved the characteristics, designating the curves along which information flows, and the streamlines designating the fluid flow paths coincide, a fact exploited by Rhines and Young (1982) to derive a theory for the vertical structure of the circulation. In the presence of buoyancy flux however, the $\mathrm{PV}$ is not conserved so that characteristics and streamlines depart from each other and it is not possible anymore to associate each streamline with a single value of potential vorticity.

There have been a number of studies based on simple conceptual models of the large-scale ocean circulation forced by buoyancy fluxes. Luyten and Stommel (1986) consider a 2-layer planetary geostrophy model where the interfacial mass flux is prescribed as function of the position, analogous to the way one specifies the Ekman pumping velocity. They determine that depending on where the characteristics originate there are two regimes of flow, the direct and the indirect cells, that differ by the sign of the vertical velocity relative to that of the interfacial flux. Pedlosky (1998) notes that the problem of nonadiabatic motion is closely related to the problem of cross-gyre flow since both involve the question of information propagation in a gyre. For a double-gyre system, the presence of nonadiabatic dynamics on the intergyre boundary determines the degree to which the two gyres can communicate (Schopp, 1993).

There have been also a number of studies of the wind- and thermally-forced ocean circulation based on 2-level models (Davey, 1983; Huang, 1993). In these models the temperature within each level can vary horizontally. The thermal forcing is represented as restoring of the surface temperature to a specified profile. Davey (1983) examines the thermally-only forced circulation on a mid-latitude beta-plane. He 
presents several analytical solutions for the large-scale baroclinic response to a thermal forcing underlying the importance of Kelvin and long internal Rossby waves in establishing the circulation. In Huang (1993) a 2-level wind- and buoyancy-forced model is presented that can be viewed as a generalization of the classical Stommel and Arons abyssal flow theory, in the sense that the vertical velocity driving the deep circulation is determined from internal dynamics rather than externally specified.

The purpose of the work presented in the remaining portion of this thesis is to examine the properties of the circulation in a simple 2-layer QG ocean contained in a closed basin, where unlike most ocean QG layer models, the circulation is driven by large-scale thermal forcing, and not by wind stress. Throughout this thesis it is assumed that the density of the fluid is entirely determined by its temperature, in which case density and buoyancy are equivalent to temperature. The 2-layer model is meant to crudely represent the warm upper waters separated from the cold abyssal waters by the ocean thermocline. Alternatively, one can think of the 2-layer model as representing the upper and lower thermocline. The presence of thermal forcing signifies that nonadiabatic processes such as vertical mixing are represented that allow for fluid parcels to cross from one density layer to the other. In other words, a non-zero cross-isopycnal velocity is allowed and is assumed to be the only driving force for the circulation. A crucial part of the model is thus the definition of the cross-isopycnal flux.

For the purpose of this study, the cross-isopycnal velocity is parameterized as restoring of the interface displacement to some prescribed equilibrium height profile. The cross-isopycnal velocity is determined thus from internal dynamics and not imposed externally as in Luyten and Stommel (1986). This is the same parameterization as the one used commonly in atmospheric QG models to represent the diabatic effects due to radiative heating (Held, 2000). In the oceanic context, the chosen parameterization implies that the changes in the surface temperature, resulting from the exchange of heat with the atmosphere, are transmitted down the water column to the thermocline by vertical mixing or other processes, leading to water property 
transformation and fluid parcel exchange between the upper and abyssal ocean. In this sense, the parameterization of the cross-isopicnal velocity bears some similarity to the Haney restoring thermal condition used in numerous GCMs, where the ocean mixed layer temperature is relaxed to some prescribed apparent atmospheric temperature (Haney, 1971). However, in our QG model it is the thermocline displacement or equivalently the vertically averaged temperature, and not the mixed layer temperature, that is restored to a prescribed equilibrium state.

A QG model with similar relaxation parameterization of the cross-isopycnal velocity has been previously examined. In Pedlosky and Spall (2005), the thermally-driven circulation on a $\beta$-plane basin is analyzed, where the applied buoyancy forcing consists not only of a vertical mixing parameterized as relaxation of the interface to a prescribed height, but also of lateral diffusion of layer thickness, representative of unresolved eddy fluxes of thickness. It is found that when processes that produce lateral diffusion of buoyancy are included, the largest vertical motions occur in a very narrow boundary layer next to the western wall. This confirms previous ideas that large-scale buoyancy forcing can lead to intense narrow regions of vertical motion next to the walls. In Pedlosky (2006), the same thermally-driven circulation is examined but for a switch-on and periodic thermal forcing. It is shown that the establishment of the circulation as well as its adjustment to changing forcing occurs through the excitation of low-frequency, weakly-damped baroclinic Rossby waves. Depending on the spatial structure of the thermal forcing, one or several Rossby waves are needed in order to reach a new equilibrium. The low-frequency, weakly-damped Rossby waves are known to be an essential ingredient of the variability of wind-driven gyres as well (Spydell and Cessi, 2003). It is worth noticing that in both these studies (Pedlosky and Spall, 2005; Pedlosky, 2006), a thermally-driven model with linear dynamics is considered, where the advection of relative vorticity and interface fluxes is neglected. 


\subsection{Goal}

In the following chapters, the circulation in a two-layer QG model confined in a closed $\beta$-plane basin is analyzed, where the sole forcing driving the circulation is a cross-isopycnal flux. We have thus completely ignored the wind stress in order to concentrate on the large-scale ocean circulation driven by heating and cooling, which in our model lead to mixing at the thermocline. The new contribution of the work presented in the second part of this thesis is that we consider a model with nonlinear dynamics, where the advection of relative and stretching vorticity is included. Our goal is to study the properties of the thermally-forced circulation when the role of the nonlinear terms, as measured by the Reynolds number, is increased. We are interested in describing and understanding the time-mean large-scale ocean circulation driven by diapicnal fluxes at the thermocline as well as its modes of variability.

In the remainder of this chapter the model equations and relevant nondimensional parameters are described in detail. Subsequently, two different regimes of the thermally-forced circulation are examined. Chapter 4 investigates the steady-state thermally-forced circulation, where the forcing is weak enough so that no instabilities occur and the circulation reaches a steady state. Chapter 5 investigates the timedependent, strongly nonlinear regime of the thermally-forced circulation. Finally, Chapter 6 deals with some questions raised from the time-dependent regime analysis.

\subsection{Definition of the model}

The simplest layered model that captures the effect of stratification is a two-layer model meant to roughly represent the warm upper ocean separated from the cold abyssal ocean by the thermocline. We consider thus a two-layer model where the two layers are described by densities $\rho_{1}$ and $\rho_{2}$ and resting depths $H_{1}$ and $H_{2}$, respectively (Figure 3-1A). The ocean is contained in a square basin of size $L$ on the $\beta$-plane, where 
the background planetary vorticity is expanded about the mid-latitude as

$$
f=f_{0}+\beta_{0}\left(y-\frac{L}{2}\right), \quad y \in[0, L]
$$

We assume that the fluid motion within each layer is governed by quasi-geostrophic dynamics which signifies that the motion departs only slightly from the linear geostrophic balance, or in other words the limit of small Rossby number is considered. The smallness of the Rossby number implies also that the vertical excursions of the interface between the two layers, representative of the ocean thermocline, are constrained to be small (order Rossby number) compared to the layer thickness (Pedlosky, 1998). In particular, layer outcropping is not allowed.

We consider initially the most general case where the ocean is forced at the surface by both wind stress and thermal relaxation forcing. The wind forcing is incorporated in the traditional way as an Ekman pumping velocity $w_{e}$ acting on the upper layer, where $w_{e}=\operatorname{curl}(\vec{\tau}) / \rho_{0} f_{0}, \vec{\tau}$ being the wind stress field. The thermal forcing leads to a cross-isopycnal velocity noted $w_{*}$, whose parameterization is described in the next section.

The motivation for the choice of this idealized framework is twofold. On the one hand, the model retains just enough dynamics so that a crude representation of the large-scale thermal forcing acting on the ocean can be considered. On the other hand, the model is simple enough and computationally efficient so that integration for long times at high resolution is realizable and practicable.

\subsubsection{The thermal forcing}

We are interested in a situation where sources of heating and cooling are present so that water can be exchanged between the two density layers. This translates into a cross-isopycnal velocity noted $w_{*}$. When $w_{*}$ is locally positive, water from the lower cold layer is transformed into warm upper layer water, or in other words the water column is heated. Inversely, when $w_{*}$ is locally negative, water from the upper warm 
A)

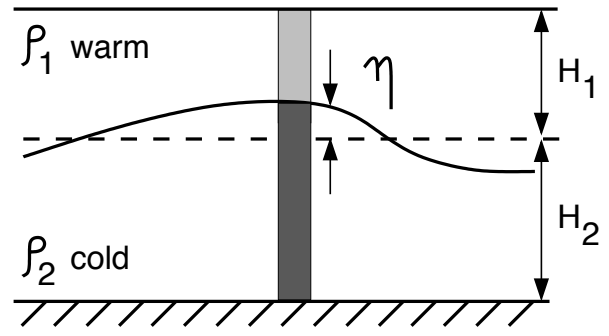

B)

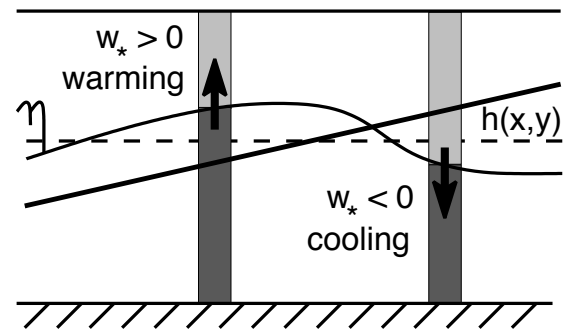

Figure 3-1: A) The upward interface displacement in a 2-layer QG model is representative of the vertically averaged thermal field - for example, $\eta$ positive means a cold anomaly. B) The cross-isopycnal velocity $w_{*}$ is parameterized as relaxation of the interface $\eta$ toward an externally specified height $h(x, y)$ on a timescale $\gamma$.

layer is transformed into cold lower layer water, or in other words the water column is cooled.

There is not an easy recipe, as with the wind forcing, that allows us to prescribe the spatial distribution of $w_{*}$ for the ocean, which is in general the result of turbulent processes depending on the local stratification and velocity field. For the purpose of this study, the cross-isopycnal velocity is parameterized as relaxation of the interface displacement $\eta$ toward some externally specified profile $h(x, y)$ on a timescale $\gamma$ (Held, 2000; Pedlosky and Spall, 2005),

$$
w_{*}=\frac{1}{\gamma}(\eta-h(x, y))
$$

Here, $\eta$ stands for the upward displacement of the interface from its rest position and can be expressed in terms of the upper and lower layer streamfunctions $\psi_{1}$ and $\psi_{2}$ as

$$
\eta=-\frac{f_{0}}{g^{\prime}}\left(\psi_{1}-\psi_{2}\right)
$$

where $f_{0}$ is the Coriolis parameter and $g^{\prime}=g \Delta \rho / \rho_{0}$, the reduced gravity. The interface displacement is proportional thus to the baroclinic part of the circulation, which can also be interpreted as the vertically averaged temperature field (Figure 3-1A). The parameterization for $w_{*}$ is therefore nothing else but a relaxation of the ver- 
tically averaged temperature toward some externally specified profile (Figure 3-1B). Hence, the chosen parameterization for the cross-isopycnal velocity $w_{*}$ can be thought of in the oceanic context as a rough representation of the vertical mixing processes that tend to restore the ocean temperature toward an equilibrium state set by the atmosphere (Pedlosky and Spall, 2005; Pedlosky, 2006). For layered atmospheric QG models, the same definition for $w_{*}$ is used in order to represent the radiative heating of the atmosphere (Held, 2000). In this case, $h$ stands instead for the radiative equilibrium temperature determined by the solar radiation.

In general, one can include in the definition (3.2) for $w_{*}$ a diffusion of the interface $\kappa \nabla^{2} \eta$ modeling the effect of the unresolved small scales, where $\kappa$ is the thermal diffusivity. Taking into account this term leads to the formation of very thin boundary layers that alter the spatial distribution of the vertical motion in a basin subject to surface cooling (Pedlosky and Spall, 2005). For all calculations presented in this thesis however, we will not include this part of the cross-isopycnal flux, i.e we will use $\kappa=0$. This choice was made since, unlike Pedlosky and Spall (2005) who consider a linear QG model, we are using a model with nonlinear dynamics, so the aim is to resolve the effect of the eddies instead of relying on a parameterization.

\subsubsection{The model equations}

The equations governing the fluid motion in a 2-layer QG model in the most general case when both wind and thermal forcing is applied are (Pedlosky, 1998)

$$
\begin{aligned}
\frac{\partial}{\partial t}\left(\nabla^{2} \psi_{1}\right)+J\left(\psi_{1}, \nabla^{2} \psi_{1}+\beta_{0} y\right) & =\frac{f_{0}}{H_{1}} w_{e}-\frac{f_{0}}{H_{1}} w_{i}+\nu \nabla^{2}\left(\nabla^{2} \psi_{1}\right), \\
\frac{\partial}{\partial t}\left(\nabla^{2} \psi_{2}\right)+J\left(\psi_{2}, \nabla^{2} \psi_{2}+\beta_{0} y\right) & =\frac{f_{0}}{H_{2}} w_{i}+\nu \nabla^{2}\left(\nabla^{2} \psi_{2}\right)-r \nabla^{2} \psi_{2}, \\
\frac{\partial \eta}{\partial t}+J\left(\psi_{n}, \eta\right) & =w_{i}-w_{*}, \\
w_{*} & =\frac{1}{\gamma}(\eta-h(x, y)), \\
\eta & =-\frac{f_{0}}{g^{\prime}}\left(\psi_{1}-\psi_{2}\right),
\end{aligned}
$$


where by definition the Jacobian $J(a, b)=(\partial a / \partial x)(\partial b / \partial y)-(\partial a / \partial y)(\partial b / \partial x)$.

We have made the choice to write the equations in a form such that the vertical velocity at the interface between the two layers $w_{i}$ appears explicitly. There are thus three unknowns: the geostrophic streamfunctions $\psi_{1}$ and $\psi_{2}$, describing the horizontal motion field by layers, and the vertical velocity $w_{i}$, describing the vertical circulation. The first three equations of the system are interpreted as the equations governing the evolution of respectively the relative vorticity for the two layers and the interface displacement $\eta$. Given that $\eta$ can also be viewed as the vertically averaged temperature field, the third equation represents as well the heat balance of the system. It states that the vertical velocity at the interface between the two layers $w_{i}$ is equal to the local rate of change of the interface $\eta$ plus the cross-isopycnal flux $w_{*}$ resulting from nonadiabatic processes.

Two dissipative mechanisms are included in the model. First of all, we have the eddy viscosity $\nu$. It represents lateral diffusion of relative vorticity and is meant to parameterize the effect of the unresolved small scales on the resolved scales. It tends to damp selectively the smallest resolved scales and plays thus the role of a numerical closure for the model. Second, we have included a Rayleigh damping coefficient $r$. It is active only in the lower layer and is meant to represent the effect of bottom stress. It can be shown that in the absence of advection the Rayleigh drag is largely scale insensitive and damps almost equally all spatial scales*. In the presence of nonlinear advection, the Rayleigh drag damps the most the largest scales. Unless otherwise specified, the dissipation coefficients are considered spatially uniform.

One can rewrite the system of equations $(3.4,3.5,3.6)$ in a form where the vertical velocity $w_{i}$ is eliminated. This leads to the following system for the evolution of the potential vorticity $Q_{n}$ by layers,

* More on the spatial scales damped selectively by the different dissipative mechanisms is given in Appendix B. 


$$
\begin{aligned}
\frac{\partial Q_{1}}{\partial t}+J\left(\psi_{1}, Q_{1}\right) & =\frac{f_{0}}{H_{1}} w_{e}-\frac{f_{0}}{H_{1}} w_{*}+\nu \nabla^{2}\left(\nabla^{2} \psi_{1}\right), \\
\frac{\partial Q_{2}}{\partial t}+J\left(\psi_{2}, Q_{2}\right) & =\frac{f_{0}}{H_{2}} w_{*}+\nu \nabla^{2}\left(\nabla^{2} \psi_{2}\right)-r \nabla^{2} \psi_{2}, \\
w_{*} & =-\frac{f_{0}}{g^{\prime} \gamma}\left(\psi_{1}-\psi_{2}+\frac{g^{\prime}}{f_{0}} h(x, y)\right), \\
Q_{n} & =\beta_{0} y+\nabla^{2} \psi_{n}+(-1)^{n} \frac{f_{0}^{2}}{g^{\prime} H_{n}}\left(\psi_{1}-\psi_{2}\right) .
\end{aligned}
$$

Here, $Q_{n}$ is the quasi-geostrophic potential vorticity for the layers equal to the combination of planetary vorticity, relative vorticity and vortex stretching of the water column. It can be seen that because of the presence of cross-isopycnal flux, the PV of the lower layer is not conserved in the inviscid limit (Pedlosky, 1998).

\subsubsection{Boundary conditions and mass conservation}

The model is assumed to be contained in a closed, flat-bottom, square basin of size $L$. No-normal flow and no-slip conditions are applied at all walls which translates into

$$
\psi_{n}=c_{n}(t), \quad \nabla \psi_{n} \cdot \hat{\mathbf{n}}=0 \quad \text { on the walls. }
$$

The no-normal flow condition requires that the streamfunction of each layer is constant along the boundary of the basin, however the constants could depend on time. The no-slip condition constrains the tangent component of the velocity to vanish at the walls. It also implies that the normal derivative of the interface displacement at the wall $\nabla \eta \cdot \hat{\mathbf{n}}$ is zero, i.e that there is no heat flux through the boundaries (when $\kappa=0)$.

The values of the streamfunctions at the walls are determined from the additional

condition of mass conservation. In order for mass to be conserved, the vertical velocity $w_{i}$ at the interface between the two layers has to integrate to zero over the area of the basin. By definition, the vertical velocity is equal to the sum of the local rate of 
change of the interface displacement $\eta$ and the cross-isopycnal velocity $w_{*}$

$$
w_{i}=\frac{\partial \eta}{\partial t}+J\left(\psi_{n}, \eta\right)+w_{*}
$$

where $\psi_{n}$ is the streamfunction of either layer since $J\left(\psi_{1}, \eta\right)=J\left(\psi_{2}, \eta\right)$. Mass conservation translates thus into the following constraint on the interface displacement

$$
\iint w_{i}(x, y, t) d x d y=0 \Longleftrightarrow \iint \frac{\partial \eta}{\partial t}+\frac{1}{\gamma}(\eta-h) d x d y=0
$$

which can be rewritten in terms of the streamfunctions as

$$
\frac{\partial}{\partial t} \iint\left(\psi_{1}-\psi_{2}\right) d x d y=-\frac{1}{\gamma} \iint\left(\psi_{1}-\psi_{2}+\frac{g^{\prime}}{f_{0}} h(x, y)\right) d x d y
$$

Suppose that at a given time $t=t_{0}$, the distribution of cold and warm layer in the model ocean is such that $\iint\left(\psi_{1}-\psi_{2}\right) d x d y=M_{0}$ and that the specified target interface displacement $h$ is time invariant. Then, the mass conservation implies that at any later time

$$
\iint\left(\psi_{1}-\psi_{2}\right) d x d y=M_{0} e^{-\frac{t-t_{0}}{\gamma}}-\left[1-e^{-\frac{t-t_{0}}{\gamma}}\right] \iint \frac{g^{\prime}}{f_{0}} h(x, y) d x d y
$$

What this equation expresses is the fact that for times much longer than the restoring timescale $\gamma$, the basin average of the temperature departure from the rest state converges toward the basin average of the externally specified temperature profile toward which the temperature is relaxed. For example, if the specified profile $h(x, y)$ is such that it integrates to a negative number over the area of the basin, this implies that net heating is applied to the system, since overall the interface between the two layers is pushed down. As a result, the mean vertical temperature integrated over the basin or equivalently the volume of the upper warm layer would increase.

In the special case when the ocean is initially at rest, i.e $\eta(x, y, t=0)=0$, and there is no net heating or cooling, i.e $\iint h(x, y) d x d y=0$, the mass conservation 
condition reduces to what is usually used in wind-only driven models

$$
\iint\left(\psi_{1}-\psi_{2}\right) d x d y=0 \text { at all times. }
$$

\subsection{Additional comments on the model}

\subsubsection{Equations by vertical modes}

Some insights about the dynamics of the 2-layer QG model with thermal forcing can be gained if the equations are rewritten by vertical modes instead of by layers.

By definition, the barotropic and baroclinic streamfunctions $\phi$ and $\tau$ are given in terms of the layer streamfunctions $\psi_{1}$ and $\psi_{2}$ by

$$
\phi=\frac{H_{1}}{H} \psi_{1}+\frac{H_{2}}{H} \psi_{2}, \quad \tau=\psi_{1}-\psi_{2}
$$

where $H=H_{1}+H_{2}$ is the full depth of the ocean. Using this definition, the potential vorticity equations by layers (3.9) and (3.10) can be transformed into potential vorticity equations by vertical modes (Salmon, 1998)

$$
\begin{aligned}
\frac{\partial Q_{\phi}}{\partial t}+J\left(\phi, Q_{\phi}\right) & +\frac{H_{1} H_{2}}{H^{2}} J\left(\tau, Q_{\tau}\right) \\
& =\frac{f_{0}}{H} w_{e}+\nu \nabla^{4} \phi-r \frac{H_{2}}{H} \nabla^{2}\left(\phi-\frac{H_{1}}{H} \tau\right), \\
\frac{\partial Q_{\tau}}{\partial t}+J\left(\phi, Q_{\tau}\right) & +J\left(\tau, Q_{\phi}+\frac{H_{2}-H_{1}}{H} Q_{\tau}\right) \\
& =\frac{f_{0}}{H_{1}} w_{e}+\frac{1}{\gamma R_{d}^{2}}\left(\tau+\frac{g^{\prime}}{f_{0}} h(x, y)\right)+\nu \nabla^{4} \tau+r \nabla^{2}\left(\phi-\frac{H_{1}}{H} \tau\right),
\end{aligned}
$$

where the potential vorticity $(\mathrm{PV})$ by vertical modes $Q_{\phi}$ and $Q_{\tau}$ is given by

$$
Q_{\phi}=\beta_{0} y+\nabla^{2} \phi, \quad Q_{\tau}=\nabla^{2} \tau-\frac{\tau}{R_{d}^{2}}
$$

and $R_{d}^{2}=g^{\prime} H_{1} H_{2} /\left(f_{0}^{2} H\right)$ is the internal Rossby deformation radius. 
The major observation that can be made from the examination of the PV equations by vertical modes is that the thermal forcing drives directly only the vertically varying part of the circulation, unlike the wind stress that generates both a barotropic and a baroclinic response. Hence, for a thermally-only driven circulation a barotropic response can be generated only through the coupling to the baroclinic part of the circulation. The coupling between $\tau$ and $\phi$ occurs either through the nonlinear term $J\left(\tau, Q_{\tau}\right)$, where advection of baroclinic PV by the baroclinic flow changes the barotropic PV, or through the bottom friction term. Note that the majority of the calculations performed in this thesis are done in a parameter regime where the bottom friction plays only a minor role in the local PV balance, its main purpose being to damp the barotropic basin modes that are sometimes excited. Thus, the barotropic circulation generated by the bottom friction coupling to the baroclinic flow is negligible, and it is mainly the nonlinear advective coupling that matters.

When analyzing a wind-only driven multi-layer model, it is useful to think about it as having a top layer directly driven by the wind stress, and deep layers that are set in motion only under certain conditions by way of parameterized or resolved eddies (Rhines and Young, 1982) or through ventilation (Pedlosky, 1998). We have seen on the other hand that for a thermally-only driven ocean, it is the baroclinic, vertically varying part of the circulation that is directly forced. We expect thus a purely baroclinic circulation in the linear regime with barotropic circulation being generated only when nonlinearities come into play. In order to underline this feature of the thermally-driven circulation, we have carried several of the analyses in the next chapters by vertical modes, and not by layers which is the traditional way.

\subsubsection{Nondimensional equations}

In order to determine a group of nondimensional parameters controlling the behavior of the wind- and thermally-forced system, it is necessary to write the equations in a nondimensional form. If $L$ is the basin length scale and $U$ a typical horizontal velocity scale, then the following nondimensional variables can be introduced, where we have 
made the choice to use a time scale based on the barotropic Rossby wave frequency

$$
(x, y)^{d i m}=L(x, y), \quad\left(\psi_{1}, \psi_{2}\right)^{\operatorname{dim}}=U L\left(\psi_{1}, \psi_{2}\right), \quad t^{d i m}=\frac{1}{\beta_{0} L} t .
$$

Note that the variables $t, x, y$ and $\psi_{n}$ stand now for the nondimensionalized quantities. Let also rewrite the forcing terms as

$$
\operatorname{curl}(\vec{\tau})=\frac{\tau_{0}}{L} F_{w}(x, y), \quad h(x, y)=h_{0} F_{T}(x, y),
$$

where $\tau_{0}$ and $h_{0}$ are typical forcing amplitudes (wind stress and interface height), while the nondimensional functions $F_{w}(x, y)$ and $F_{T}(x, y)$ define their spatial variation.

The PV equations by vertical modes become then in nondimensional form

$$
\begin{aligned}
\frac{\partial q_{\phi}}{\partial t}+\delta_{I}^{2}\left[J\left(\phi, q_{\phi}\right)\right. & \left.+\delta(1-\delta) J\left(\tau, q_{\tau}\right)\right]+\frac{\partial \phi}{\partial x}=\delta U_{w} F_{w} \\
& +\delta_{M}^{3} \nabla^{4} \phi-\delta_{S}(1-\delta) \nabla^{2}(\phi-\delta \tau) \\
\frac{\partial q_{\tau}}{\partial t}+\delta_{I}^{2}\left[J\left(\phi, q_{\tau}\right)+J\left(\tau, q_{\phi}\right)+(1-2 \delta) J\left(\tau, q_{\tau}\right)\right]+\frac{\partial \tau}{\partial x} & =U_{w} F_{w} \\
+\frac{1}{\delta_{T}}\left(\tau+U_{T} F_{T}\right)+\delta_{M}^{3} \nabla^{4} \tau & +\delta_{S} \nabla^{2}(\phi-\delta \tau) \\
q_{\phi} & =\nabla^{2} \phi \\
q_{\tau} & =\nabla^{2} \tau-F \tau
\end{aligned}
$$

The equations are supplemented with the no normal flow and no-slip boundary conditions $\phi=0, \tau=c(t)$ and $\nabla \phi \cdot \hat{\mathbf{n}}=\nabla \tau \cdot \hat{\mathbf{n}}=0$ on the walls, and the nondimensionalized mass conservation which is, in its most general form,

$$
\frac{\partial}{\partial t} \iint \tau d x d y=-\frac{1}{F \delta_{T}} \iint\left(\tau+U_{T} F_{T}\right) d x d y
$$

A set of eight nondimensional parameters characterize the system. First, we have 
a number of parameters describing the geometry and forcing:

$$
F=\left(\frac{L}{R_{d}}\right)^{2}, \quad \delta=\frac{H_{1}}{H}, \quad U_{w}=\frac{1}{U}\left(\frac{\tau_{0}}{\rho_{0} \beta_{0} H_{1} L}\right), \quad U_{T}=\frac{1}{U}\left(\frac{g^{\prime} h_{0}}{f_{0} L}\right)
$$

The rotational Froude number $F$ measures the ratio of the basin size to the internal Rossby deformation radius $R_{d}$, or equivalently the importance of vortex stretching to inertia. The parameter $\delta$ is a geometrical parameter equal to the ratio of the upper layer rest depth to the full ocean depth. The parameters $U_{w}$ and $U_{T}$ represent the nondimensionalized horizontal velocity scales associated with the wind and thermal forcing, respectively. Depending on whether the circulation is wind or thermally driven, one of these parameters is used to define the typical velocity scale $U$ of the flow: $U=\tau_{0} / \rho_{0} \beta_{0} H_{1} L$ for wind-driven ocean, or $U=g^{\prime} h_{0} / f_{0} L$ for thermally-driven ocean. If both wind and thermal forcing are applied, then one can use the windderived velocity scale $U$ with the nondimensional parameter $U_{T}$ representing in this case the ratio of the thermally to the wind driven part of the circulation.

There are also several parameters associated with characteristic length scales of the system:

$$
\delta_{I}=\frac{1}{L}\left(\frac{U}{\beta_{0}}\right)^{\frac{1}{2}}, \quad \delta_{M}=\frac{1}{L}\left(\frac{\nu}{\beta_{0}}\right)^{\frac{1}{3}}, \quad \delta_{S}=\frac{1}{L}\left(\frac{r}{\beta_{0}}\right), \quad \delta_{T}=\frac{1}{L}\left(\gamma \beta_{0} R_{d}^{2}\right)
$$

Here, $\delta_{I}$ is the inertial boundary layer width, $\delta_{M}$ the Munk boundary layer width, and $\delta_{S}$ the Stommel boundary layer width, measuring respectively the relative importance of the nonlinear advection, lateral dissipation and bottom damping of relative vorticity to the advection of planetary vorticity*. Finally, $\delta_{T}$ is the thermal lengthscale indicative of the strength of the thermal relaxation. What this lengthscale actually represents is the ratio of the distance travelled by the long internal Rossby waves with speed $c_{R}=\beta_{0} R_{d}^{2}$ during the relaxation timescale $\gamma$, to the basin scale $L$. More on the choice of the thermal forcing parameters, including the restoring time scale, are

${ }^{*}$ It is sometimes useful to refer to the dimensional boundary layer widths which will be denoted by a tilde with $\tilde{\delta}_{I}, \tilde{\delta}_{M}, \tilde{\delta}_{S}$ and $\tilde{\delta}_{T}$, and are simply equal to the respective non-dimensional version multiplied by the length scale $L$. 
given in the next section.

The behavior of the wind- and thermally-forced system is controlled therefore by a set of 7 nondimensional parameters. For the scope of this thesis, the dependence of the circulation on only a small number of these parameters is examined. The central focus is put on the dependence of the resulting circulation on the inertial boundary layer width, or in other words the changes that occur in the time-mean circulation and its variability when the role played by the nonlinearities increases. The importance of the bottom drag is also examined, while some other effects are completely ignored. For example, the majority of the results in this thesis concern a thermally-only forced ocean, thus $U_{w}=0$. More details on the specific parameter values are given when the different calculations are presented.

\subsubsection{Choice of the thermal relaxation forcing}

\section{The thermal relaxation profile}

The function $F_{T}(x, y)$, that specifies the spatial variation of the target height toward which the interface is relaxed, can be thought as the equilibrium temperature distribution toward which the vertically integrated ocean temperature is restored by vertical mixing processes.

In all the cases that are discussed in this thesis, the thermal forcing is chosen so that it integrates to zero over the basin. That means that if the upper water column is squeezed at some location, then it is expanded at some other, so that overall the amount of warm and cold water in the system remains the same as initially specified by the rest depths $H_{1}$ and $H_{2}$. In other words, no net heating/cooling is applied to the system. This guarantees, among other things, that an equilibrium can be reached.

If on the other hand the thermal forcing is such that $\iint F_{T} d x d y \lessgtr 0$, then net heating/cooling is exerted. The system will respond to this forcing by adjusting the amount of warm and cold water specified initially. This will modify the rest depths $H_{1}$ and $H_{2}$, until $\iint F_{T} d x d y=0$ respective to the "new" rest depths. Thus, in general 

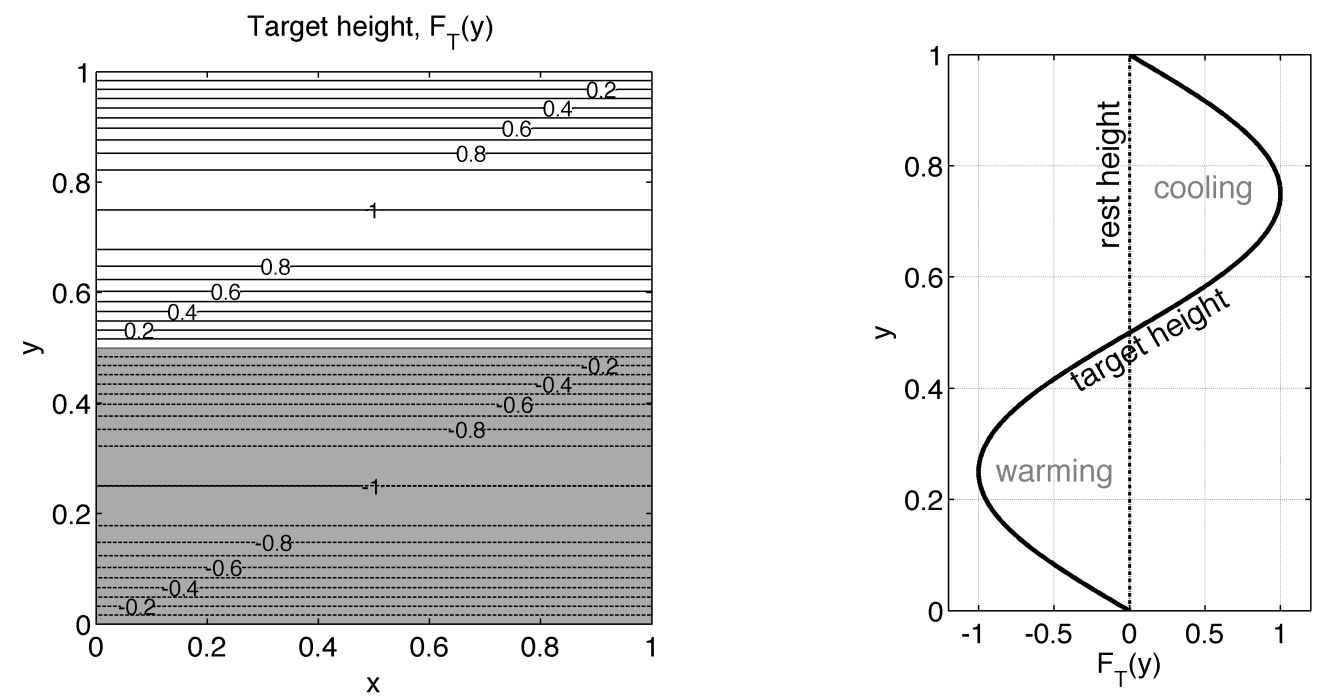

Figure 3-2: The spatial structure of the profile toward which the interface is relaxed. $F_{T}(x, y)$ is chosen uniform in the zonal direction and with a sine dependence on latitude, corresponding to warming in the southern half and cooling in the northern half of the basin. There is no net heat flux applied to the system.

applying a thermal forcing with net heating/cooling is equivalent to applying a no net heat flux thermal forcing, but for some different rest depths $H_{1}$ and $H_{2}$, given of course that the specified amount of heating/cooling is physically meaningful, i.e. it does not deplete any of the layers for example.

Finally, there is also the special case where $F_{T}(x, y)=0$, which means that the interface is relaxed toward the rest depth. In this case, a circulation will result only if wind forcing is also applied. The "thermal forcing" acts then as an interfacial friction - it tends to flatten the isopycnals and damps the circulation.

All calculations performed in this thesis are done with a target interface displacement $F_{T}(y)$ that is taken uniform in the zonal direction and antisymmetric with respect to the mid-latitude, as shown in Figure 3-2. The variation with latitude is given by a sine function and is such that the southern half of the basin is warmed while the northern half is cooled. This is meant to roughly represent the large-scale thermal forcing acting on the subtropical and subpolar gyres in the real ocean.

The particular choice of a sine profile was made for the following reason. While the 
circulation in the interior of the basin is qualitatively very similar no matter what the exact form of the target profile is, as long as it corresponds to the same general pattern of heating in the south and cooling in the north, having a non-zero thermal forcing at the northern and southern boundaries forces strong zonal boundary layers where the meridional velocity is brought to zero. We have chosen thus to bring the thermal forcing to zero at the northern and southern boundaries by using a sine function for $F_{T}(y)$ in order to emphasize on the interior dynamics of a thermally-forced ocean. The same sine target profile for the thermal forcing is used for all calculations analyzed in this thesis, with only its amplitude varied between the different calculations.

\section{The thermal relaxation timescale}

One way to justify the choice of the relaxation timescale $\gamma$ is using the thermal lengthscale $\delta_{T}$, defined as the ratio of the relaxation timescale $\gamma$ to the time $T_{R}=$ $L / \beta_{0} R_{d}^{2}$ needed for a long internal Rossby wave to cross the basin. Long internal Rossby waves play a central role in the establishment of the vertical structure of the circulation. They are messengers carrying the signal of the blocking action of the eastern boundary (Pedlosky, 1998). A strong enough eastward barotropic flow can arrest their westward propagation creating regions of closed geostrophic contours and homogenized potential vorticity isolated from the eastern wall (Rhines and Young, 1982). The transit time $T_{R}$ is thus an important internal timescale of the problem.

Adding a large-scale thermal forcing parameterized as relaxation of the interface displacement tames the propagation of internal Rossby waves on the interface between the two layers. Two limits can be envisioned:

i) For timescales $\gamma$ short compared to $T_{R}$, the Rossby waves are strongly affected by the interface relaxation and cannot reach too far away from the eastern boundary, failing thus to propagate information all the way across the basin. This is the limit of strong relaxation and corresponds to $\delta_{T} \ll 1$.

ii) For timescales $\gamma$ long compared to $T_{R}$, the Rossby waves are able to cross 
the basin without being damped by the interface relaxation. Information is propagated in this case from the eastern boundary all the way to the western wall in the absence of advection. This is the limit of weak relaxation and corresponds to $\delta_{T} \gtrsim 1$.

Another way to interpret the relaxation timescale $\gamma$ is by making a connection to a corresponding heat flux. The presence of cross-isopycnal flux is directly related to the presence of internal sources of heating and cooling in the fluid. In particular, it can be shown that for a continuously stratified fluid with no diffusivity and a linear equation of state, where $z$ indicates the height of a surface of constant density and $w$ the vertical velocity, the cross-isopycnal flux $w_{*}$ is equal to (Pedlosky, 1998)

$$
w_{*}=w-\left[\frac{\partial z}{\partial t}+\vec{u} \cdot \nabla z\right]=\frac{\alpha Q}{H c_{p}(-\partial \rho / \partial z)} .
$$

Here, $\alpha$ is the thermal expansion coefficient, $c_{p}$ the specific heat at constant pressure, and $Q$ the heat added locally to the fluid. Given the definition (3.2) of the parameterized cross-isopycnal velocity used in our 2-layer QG model, a relationship between the heat flux $Q$ and the relaxation time scale $\gamma$ can be deduced

$$
\gamma=\frac{c_{p} \Delta \rho h_{0}}{\alpha Q} .
$$

Note however, than the heat flux $Q$ here is an internal heat flux applied at the thermocline separating the upper warm ocean and the cold abyssal ocean. It is not clear what represents a typical or reasonable value for this heat flux or neither how it can be related to the surface heat flux, which a measurable quantity.

Therefore, within this thesis we have chosen the values for the relaxation time scale $\gamma$ by comparing it to the Rossby wave transit time $T_{R}$. In particular, only the case of weak relaxation when the Rossby waves are weakly damped and are able to carry information all the way across the basin is examined. This signifies that the thermal lengthscale is on the order of the basin or larger, i.e $\delta_{T}=\gamma / T_{R} \gtrsim 1$. The case with $\delta_{T}<1$ is also interesting but because of the large number of nondimensional 

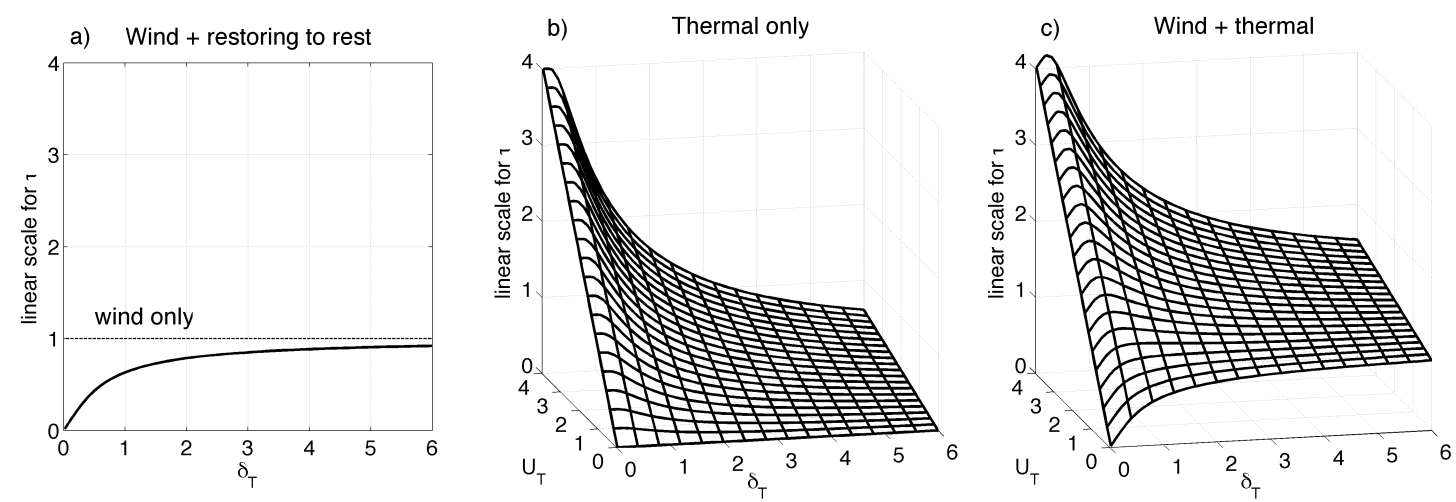

Figure 3-3: Scaling for the vertically-varying part of the circulation determined from a linear baroclinic potential vorticity balance for the cases of a) wind-forced circulation with interface restoring to rest, b) thermally-only forced circulation, and c) combined wind- and thermally-forced circulation. In all three cases $U_{w}=1$.

parameters affecting the system behavior, this case was left for a future study.

\section{Circulation scaling in the linear limit}

In order to further illustrate how the different parameters defining the relaxation thermal forcing affect the resulting circulation, a simple scaling argument for the horizontal velocity scale is presented.

Consider the steady linear inviscid equation for the baroclinic circulation where the vorticity input by wind and thermal forcing is balanced by advection of planetary vorticity

$$
\frac{\partial \tau}{\partial x}=U_{w} F_{w}(y)+\frac{U_{T}}{\delta_{T}} F_{T}(y)+\frac{\tau}{\delta_{T}}
$$

Its solution is

$$
\tau(x, y)=\left(\delta_{T} U_{w} F_{w}(y)+U_{T} F_{T}(y)\right)\left(e^{\frac{x-1}{\delta_{T}}}-1\right)
$$

which leads to the following scaling for the baroclinic streamfucntion

$$
\tau \sim\left(\delta_{T} U_{w}+U_{T}\right)\left(1-e^{-\frac{1}{\delta_{T}}}\right)
$$


Three different cases can be considered. If $U_{T}=0$, the circulation is forced by wind stress while the interface between the layers is restored to its rest position. In this case, strong restoring ( small $\delta_{T}$ ) damps the circulation, while in the limit of weak restoring (large $\delta_{T}$ ), the purely wind-driven case is recovered. If the velocity scale for the problem $U$ is chosen to be the one determined from the wind stress, i.e. $U_{w}=1$, then in the limit $\delta_{T} \rightarrow \infty, \tau$ approaches 1 as shown in Figure 3-3a.

If instead, $U_{w}=0$ and $U_{T}$ is different than zero, then the circulation is thermallyonly forced. In this case, increasing $U_{T}$, while keeping the same relaxation $\delta_{T}$, leads to a stronger circulation (Figure 3-3b). On the other hand, keeping the same $U_{T}$ but considering a weaker relaxation by increasing $\delta_{T}$, leads to a weaker circulation.

Finally, the scaling for the combined wind- and thermally-forced circulation is a superposition of the wind-only and thermally-only forced cases (Figure 3-3c). For the same restoring timescale $\delta_{T}$, increasing $U_{T}$ leads to a more thermally dominated circulation. For the same $U_{T}$, increasing $\delta_{T}$, i.e. imposing weaker relaxation, leads to a wind dominated circulation.

The calculations presented in this thesis are done with $\delta_{T}$ chosen between 1 and 1.5. This was previously described as weak relaxation in the sense that the restoring timescale $\gamma$ is long enough, so that a long internal Rossby wave can cross the basin without being damped by the relaxation. The scaling argument presented here shows that $\delta_{T}=1.5$ is small enough so that if wind forcing was included, both the wind and the thermal forcing will contribute in significant amounts to the circulation.

\subsubsection{Numerics}

The 2-layer QG model used for the time-stepping simulations performed in this thesis is a modification of a model written by P. Berloff and used in numerous studies, e.g. (Berloff and McWilliams, 1999a; Berloff and Meacham, 1998). The major changes made to the code include the implementation of the thermal forcing and the subsequent modification of the mass conservation constraint to its more general form (3.29) in the presence of heating/cooling. 
The method of solving the equations is essentially the same as outlined in Holland (1978). The equations are discretized using 2 nd order finite differences with an Arakawa Jacobian for the advective terms Arakawa (1997). A 2nd order leap-frog scheme is used for the time stepping of the vorticity with averaging between the time steps for numerical stability. A direct fast sine transform algorithm is used to solve the elliptic problems that provide the streamfunction from the relative vorticity. After each time step the mass conservation condition is applied to the baroclinic streamfunction. The no-slip boundary conditions are implemented using a 2nd order scheme (Verron and Blayo, 1996; Jensen, 1959). An uniform grid is used with sufficient resolution so that the frictional boundary layers are resolved by at least one cell. 


\section{Chapter 4}

\section{Thermally-forced ocean in the steady regime}

In this chapter the focus is on the thermally-forced circulation when the final state reached by the system is a steady equilibrium. It is demonstrated that although in the linear limit the thermal forcing drives a purely baroclinic circulation, when the nonlinear advection of relative vorticity becomes important a vertically integrated circulation is generated through the nonlinear coupling to the baroclinic flow. It is discussed how the circulation, vorticity and heat balance change with the Reynolds number. The results from the steady regime are used later as a reference for analyzing the time-dependent, strongly nonlinear, thermally-forced circulation.

\subsection{Model setup}

The values of the model parameters used in the steady regime calculations are given in Table 4.1.

All the simulations in this chapter are done in a relatively small square basin with width $L=1024 \mathrm{~km}$ but the circulation behavior is similar in larger size basins. The model is forced with a thermal forcing that has a target height $h(x, y)$ with a sine dependence on latitude, corresponding to warming over the southern half-basin 


\begin{tabular}{|c|c|c|c|}
\hline \multicolumn{2}{|c|}{ Dimensional parameters } & \multicolumn{2}{|c|}{ Nondimensional parameters } \\
\hline$L_{x}, L_{y}$ & $1024 \mathrm{~km}$ & $\delta$ & 0.17 \\
\hline$H_{1}$ & $500 \mathrm{~m}$ & $F$ & 1200 \\
\hline$H_{2}$ & $2500 \mathrm{~m}$ & $\tilde{\delta}_{M}\left(\delta_{M}\right)$ & $21.33 \mathrm{~km}(0.021)$ \\
\hline$f_{0}$ & $1 \times 10^{-4} s^{-1}$ & $\tilde{\delta}_{S}\left(\delta_{S}\right)$ & $0.05 \mathrm{~km}\left(5 \times 10^{-5}\right)$ \\
\hline$\beta_{0}$ & $2 \times 10^{-11} m^{-1} s^{-1}$ & $\tilde{\delta}_{T}\left(\delta_{T}\right)$ & $1440 \mathrm{~km}(1.406)$ \\
\hline$\rho_{0}$ & $1000 \mathrm{kgm}^{-3}$ & & \\
\hline$R_{d}$ & $30 \mathrm{~km}$ & & \\
\hline$g^{\prime}$ & $0.02 \mathrm{~ms}^{-2}$ & & \\
\hline$\nu$ & $194 m^{2} s^{-1}$ & Grid an & solution \\
\hline$r$ & $1 \times 10^{-9} s^{-1}$ & grid & $257 \times 257$ \\
\hline$\gamma$ & 2.5 years & $\Delta x$ & $4 \mathrm{~km}$ \\
\hline
\end{tabular}

Table 4.1: Values of the parameters used in the steady regime calculations. The left column defines the dimensional parameters. The right column gives the values of the nondimensional parameters and the grid resolution.

and cooling over the northern half-basin with no net heat flux into the system. The relaxation timescale $\gamma=2.5$ years is slightly longer than the time needed for a long internal Rossby wave to cross the basin $T_{R}=L / \beta_{0} R_{d}^{2} \approx 1.8$ years, which leads to a thermal lengthscale $\delta_{T}>1$ or, as discussed in the previous chapter, a case of weak thermal relaxation.

An eddy viscosity $\nu=194 \mathrm{~m}^{2} \mathrm{~s}^{-1}$ is applied that corresponds to a Munk boundary layer width $\tilde{\delta}_{M}=21 \mathrm{~km}$. Given that a grid resolution $\Delta x=4 \mathrm{~km}$ is used, the boundary layer is well resolved. The Stommel boundary layer on the other hand is three orders of magnitude smaller, corresponding to a bottom friction decay rate $r=10^{-9} s^{-1}$, or equivalently to a frictional spin-down timescale $1 / r \approx 32$ years. Thus, we are considering the regime where $\delta_{S} \ll \delta_{M}$, implying a circulation closed through a Munk western boundary layer, either linear or nonlinear depending on how important the inertial terms are.

It is the amplitude of the thermal forcing that determines the size of the inertial terms. A series of three different values for the amplitude of the target interface displacement are used. Those values, as well as the corresponding inertial boundary 


\begin{tabular}{clll}
\hline \hline$h_{0}$ & $2 \mathrm{~m}$ & $50 \mathrm{~m}$ & $120 \mathrm{~m}$ \\
\hline$U$ & $0.04 \mathrm{cms}^{-1}$ & $0.98 \mathrm{cms}^{-1}$ & $2.34 \mathrm{cms}^{-1}$ \\
$\tilde{\delta}_{I}\left(\delta_{I}\right)$ & $4.5 \mathrm{~km}(0.004)$ & $22.1 \mathrm{~km}^{(0.022)}$ & $34.2 \mathrm{~km}(0.033)$ \\
$R e=\delta_{I}^{2} / \delta_{M}^{3}$ & 2.1 & 51.7 & 123.5 \\
\hline \hline
\end{tabular}

Table 4.2: Values for the amplitude of the interface displacement used in the steady regime calculations. Given are also the corresponding values of the velocity scale $U$, the inertial boundary layer width $\delta_{I}$, and the Reynolds number $R e$. The velocity scale is chosen so that $U_{T}=1$, i.e. $U=g^{\prime} h_{0} / f_{0} L$.

layer widths, are given in Table 4.2. The inertial boundary layer $\tilde{\delta}_{I}$ is varied from $4.5 \mathrm{~km}$, which is several times smaller than the Munk layer, to $34.2 \mathrm{~km}$, which is larger than the Munk layer. The goal is to span a range of regimes where the inertial terms become more and more important, as reflected by the increasing values for the Reynolds number, $R e=\delta_{I}^{2} / \delta_{M}^{3}=U L / \nu$.

For all simulations we are using a time-stepping model where the final state to be analyzed is obtained after 60 years of integration starting from a rest state. As a reminder, in all cases examined in this chapter the final state reached by the system is a steady equilibrium. Thus, although the nonlinear terms become more important as the forcing amplitude is increased, they do not lead to instability and time-dependence of the circulation for the range of Reynolds numbers considered here. For reference, the circulation for a forcing amplitude $h_{0}=132 \mathrm{~m}$ or $R e=136.2$ is time-dependent. The question of the destabilization of a thermally-forced circulation will be addressed in Chapter 6.

\subsection{Overview of the circulation}

Before looking at the circulation patterns as the Reynolds number is varied, it is useful to explore the linear limit of the vorticity equations governing the system dynamics.

In the limit of weak forcing, the inertial boundary layer width is small and the nonlinear advection terms in the vorticity equations (3.25) and (3.26) can be neglected. 
Given also that we are dealing with a steady, thermally-only forced ocean with no vorticity input from wind stress, the potential vorticity balance in the interior of the basin reduces to

$$
\begin{aligned}
\frac{\partial \phi}{\partial x}=0 & \Rightarrow & \phi(x, y)=0, \\
\frac{\partial \tau}{\partial x}=\frac{\tau+U_{T} F_{T}(y)}{\delta_{T}} \equiv-F w_{*} & \Rightarrow & \tau(x, y)=U_{T} F_{T}(y)\left(e^{\frac{x-1}{\delta_{T}}}-1\right) .
\end{aligned}
$$

The linear PV balance for the vertically integrated circulation given by Eq.(4.1) is simple. Since there is no barotropic vorticity input from external forcing, there is no resulting barotropic circulation. Indeed, for the most weakly forced case where $h_{0}=$ $2 m$ and $\tilde{\delta}_{I}=4.6 \mathrm{~km}$ shown in Figure 4-1(A), it can be seen that there is practically no barotropic circulation except for a very weak flow in the western boundary layer ( $\max \phi=0.0031$ compared to $\max \tau=1.0834$ ). The reason for this flow is the weak, but not negligible contribution from the nonlinear advective terms in the boundary layer.

The linear PV balance for the baroclinic circulation in the interior of the basin given by Eq.(4.2) is also simple. It represents a balance between the input of baroclinic vorticity by the relaxation thermal forcing and the baroclinic advection of background planetary vorticity (Pedlosky and Spall, 2005). For example, in the northern half of the basin where $F_{T}(y)$ is positive, the water column is cooled which raises the interface between the two layers. This leads to vortex squeezing or a positive vorticity input for the upper layer, and a vortex stretching or negative vorticity input for the lower layer. To compensate for that, the fluid moves north in the upper layer, and south in the lower layer, toward higher/lower planetary vorticity, respectively. The opposite happens in the southern gyre, where warming is applied. When no cross-boundary flow is allowed, a double gyre baroclinic circulation develops, as shown in Figure 41(D), where the southern gyre rotates clockwise and the northern gyre, anti-clockwise for the upper layer. The linear thermally-forced circulation is thus similar to the linear circulation resulting from a typical mid-latitude wind stress with the one difference 


$$
\begin{gathered}
\tilde{\delta}_{I}=4.5 \mathrm{~km} \\
\left(\tilde{\tau}^{(l i n)}=0.02 \times 10^{4} \frac{\mathrm{m}^{2}}{\mathrm{~s}}\right)
\end{gathered}
$$

A) Barotropic streamfunction
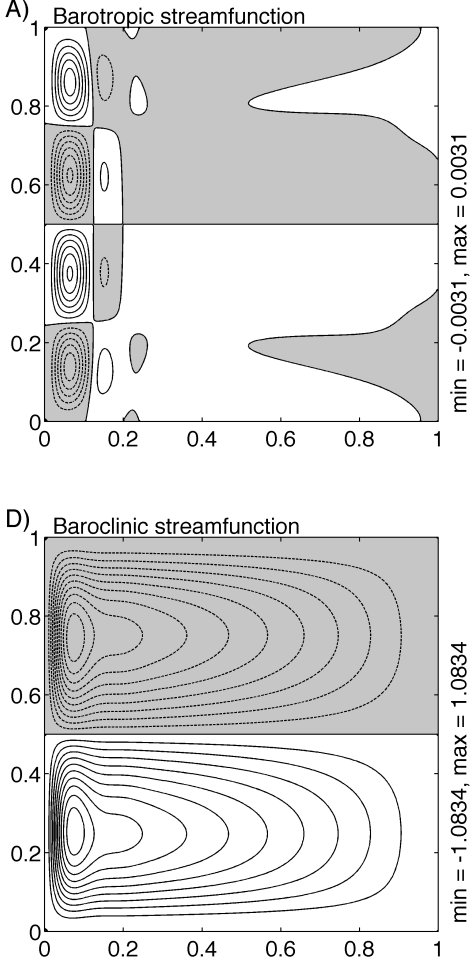

$\tilde{\delta}_{I}=22.1 \mathrm{~km}$

$\left(\tilde{\tau}^{(l i n)}=0.55 \times 10^{4} \frac{m^{2}}{s}\right)$
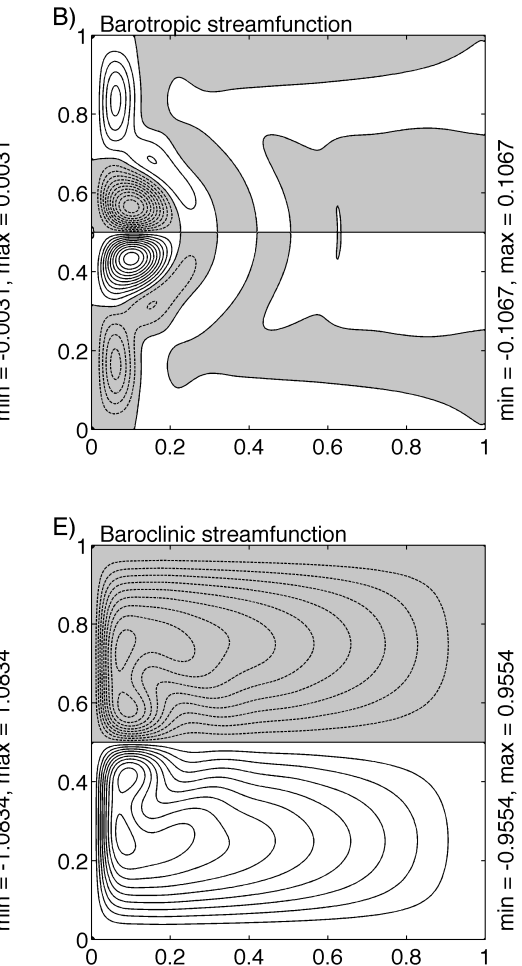

$$
\begin{gathered}
\tilde{\delta}_{I}=34.2 \mathrm{~km} \\
\left(\tilde{\tau}^{(l i n)}=1.32 \times 10^{4} \frac{\mathrm{m}^{2}}{\mathrm{~s}}\right)
\end{gathered}
$$
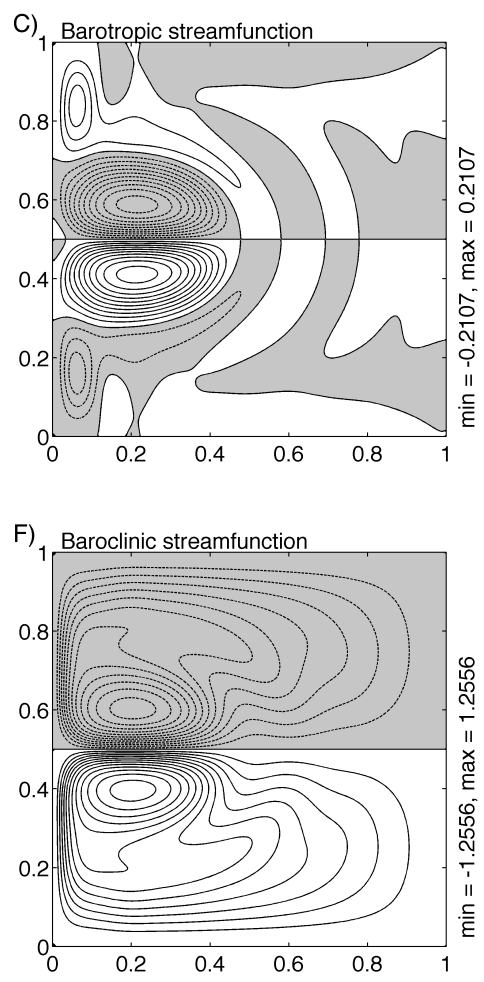

Figure 4-1: Circulation in the steady regime as a function of the inertial boundary layer thickness. Panels A-B-C, barotropic streamfunction, and panels D-E-F baroclinic streamfunction, for the case respectively of $\tilde{\delta}_{I}=4.5,22.1$ and $34.2 \mathrm{~km}$. In each case, the solutions are scaled with the maximum linear baroclinic streamfunction $\tilde{\tau}^{(l i n)}=\frac{g^{\prime} h_{0}}{f_{0}}\left(1-e^{-\frac{1}{\delta_{T}}}\right)$. The contour interval for the barotropic streamfunction is $0.0005,0.01$ and 0.02 , respectively. The contour interval for the baroclinic streamfunction is 0.1 . Regions of negative values are shaded.

that there is no barotropic component.

The maximum baroclinic streamfunction in the linear limit, $\tau^{(l i n)}=U_{T}\left(1-e^{-\frac{1}{\delta_{T}}}\right)$, depends on both the amplitude of the thermal forcing through $U_{T}$, and the restoring timescale through $\delta_{T}$, with the later especially important in the weak restoring limit when $\delta_{T} \gtrsim 1$. The dimensional version of the maximum linear transport $\tilde{\tau}^{(\text {lin })}=$ $\tau^{(l i n)} U L$ is used throughout the thesis as a scale when plotting both the barotropic 
and baroclinic components of the circulation. Values in excess of one for the baroclinic circulation in the interior of the basin indicate nonlinearly driven flow.

When the amplitude of the forcing is increased, the inertial boundary layer grows. For an amplitude of the target interface $h_{0}=50 \mathrm{~m}$, the inertial boundary layer is $\tilde{\delta}_{I}=22.1 \mathrm{~km}$, which is comparable to the Munk boundary layer, $\tilde{\delta}_{M}=21.3 \mathrm{~km}$. For an amplitude of the target interface $h_{0}=120 \mathrm{~m}$, the inertial boundary layer is $\tilde{\delta}_{I}=34.2 \mathrm{~km}$ and exceeds the Munk boundary layer. The increased importance of the nonlinear advection leads to changes in the flow pattern. It can be seen in Figure 4-1(B-C, E-F) that in the western part of the basin inertial recirculation gyres form. They are on the sub-basin scale, increase in size as $\delta_{I}$ gets larger, and have both barotropic and baroclinic component. The magnitude of the baroclinic circulation for the most strongly forced case surpasses one, i.e. the maximum linear value, indicating substantial role played by the nonlinearities. The magnitude of the barotropic flow increases with increasing Reynolds number but remains at least an order weaker than that of the baroclinic flow. The appearance of recirculation gyres as the circulation becomes inertially dominated is common in wind-driven gyres as well.

Next, the potential vorticity balances for the thermally-forced circulation are analyzed. Specific questions of interest are:

1. What drives barotropic circulation in the nonlinear steady regime?

2. What is the baroclinic vorticity balance and what drives the recirculations?

3. What is the heat budget for the system?

4. Are there any substantial differences from the wind-driven case?

\subsection{Dynamics of the barotropic circulation}

Because of the lack of barotropic vorticity input by the thermal relaxation forcing, there is no interior vertically integrated circulation in the linear limit for a thermallyonly forced ocean. However, it was determined that when the nonlinear advection of 
relative vorticity becomes important, a vertically integrated circulation is generated. Examining the potential vorticity balance for the barotropic vertical mode can help identify what drives this circulation.

When the inertial boundary layer thickness becomes comparable to that of the Munk layer, the vertically integrated circulation is governed by the steady, non-linear vorticity equation, obtained from Eq.(3.25) by eliminating the time-dependence

$$
\delta_{I}^{2}\left[J\left(\phi, \nabla^{2} \phi\right)+\delta(1-\delta) J\left(\tau, \nabla^{2} \tau\right)\right]+\frac{\partial \phi}{\partial x}=\delta_{M}^{3} \nabla^{4} \phi-\delta_{S}(1-\delta) \nabla^{2}(\phi-\delta \tau)
$$

A convenient way to interpret this equation is to note that every single term in it represents a divergence of a vorticity flux (Fox-Kemper, 2003). More specifically, the equation can be rewritten as,

$$
0=\nabla \cdot[-\underbrace{\phi \hat{\mathbf{x}}}_{\begin{array}{c}
\text { plane- } \\
\text { tary }
\end{array}}-\underbrace{\delta_{I}^{2} \mathbf{u}_{\phi} \nabla^{2} \phi}_{\begin{array}{c}
\text { barotropic- } \\
\text { barotropic }
\end{array}}-\underbrace{\delta_{I}^{2} \delta(1-\delta) \mathbf{u}_{\tau} \nabla^{2} \tau}_{\begin{array}{c}
\text { baroclinic- } \\
\text { baroclinic }
\end{array}}+\underbrace{\delta_{M}^{3} \nabla\left(\nabla^{2} \phi\right)-\delta_{S}(1-\delta) \nabla(\phi-\delta \tau)}_{\text {frictional }}],
$$

where $\mathbf{u}_{\phi}$ and $\mathbf{u}_{\tau}$ are the barotropic and baroclinic velocity fields, defined in terms of the streamfunction as $\mathbf{u}_{\psi}=(-\partial \psi / \partial y, \partial \psi / \partial x)$ with $\psi=\phi, \tau$, respectively.

Furthermore, it is helpful to integrate the vorticity equation over a region $C_{\phi}$ enclosed by a barotropic streamline. Using the divergence theorem allows us then to transform the vorticity flux divergences into vorticity fluxes across the bounding streamline. Because the barotropic velocity is by definition aligned with the barotropic streamlines, there is no contribution from the planetary vorticity and the barotropic-barotropic advective terms to the cross-streamline flux. The following budget is thus obtained

$$
0=\oint_{\partial C_{\phi}}\left[-\delta_{I}^{2} \delta(1-\delta) \mathbf{u}_{\tau} \nabla^{2} \tau+\delta_{M}^{3} \nabla\left(\nabla^{2} \phi\right)-\delta_{S}(1-\delta) \nabla(\phi-\delta \tau)\right] \cdot \hat{\mathbf{n}} d l
$$

In the absence of wind stress, there is no input of barotropic vorticity from external forcing and all the different fluxes accomplish is to redistribute vorticity within the 
region $C_{\phi}$. In particular, the cross-streamline fluxes due to the baroclinic-baroclinic advection and the friction have to balance out.

In Figure 4-2, the barotropic vorticity budget has been diagnosed for a series of regions with progressively larger area lying in the northern recirculation gyre for the most strongly forced case with $h_{0}=120 \mathrm{~m}$ or $\tilde{\delta}_{I}=34.2 \mathrm{~km}$. This is the steady circulation distinguished with the largest recirculations and strongest barotropic flow. As shown in the Figure, the northern recirculation is characterized with positive barotropic relative vorticity. The vorticity budget is essentially the same no matter which bounding streamline is chosen. It shows that barotropic vorticity is carried into the region by the baroclinic-baroclinic advective term, and carried out mostly by lateral friction, and to much smaller almost negligible extent by bottom friction. Hence, the barotropic recirculations are driven by the coupling to the baroclinic, directly thermally-forced part of the circulation.

The strongest barotropic circulation is expected in the regions where the strongest cross-streamline baroclinic-baroclinic advective flux occurs. From the integrated vorticity budget given by Eq.(4.5) it is clear that two conditions need to be met in order to have a large integrated baroclinic-baroclinic flux. First, there should be a large amount of baroclinic vorticity to be advected. Second, the barotropic and baroclinic streamlines should not be aligned, i.e $\mathbf{u}_{\tau} \cdot \mathbf{u}_{\phi} \neq 0$ so that there is a cross-streamline flux.

In Figure 4-3 the baroclinic vorticity, $\nabla^{2} \tau$, and the divergence of the baroclinicbaroclinic vorticity flux, $-\nabla \cdot\left(\mathbf{u}_{\tau} \nabla^{2} \tau\right)$ are plotted for the three cases $\tilde{\delta}_{I}=4.5,22.1$ and $34.2 \mathrm{~km}$ corresponding to $h_{0}=2,50$ and $120 \mathrm{~m}$, respectively. Only the western side of the northern half-basin is shown in order to emphasize the western boundary layer and the recirculation gyre, if present. Similar dynamics occur in the southernhalf basin, except for some sign reversals.

As expected, the western boundary layer is characterized with large amount of baroclinic relative vorticity. It is negative right next to the wall because of the no-slip boundary condition, while it is positive in the outer edge of the western boundary 

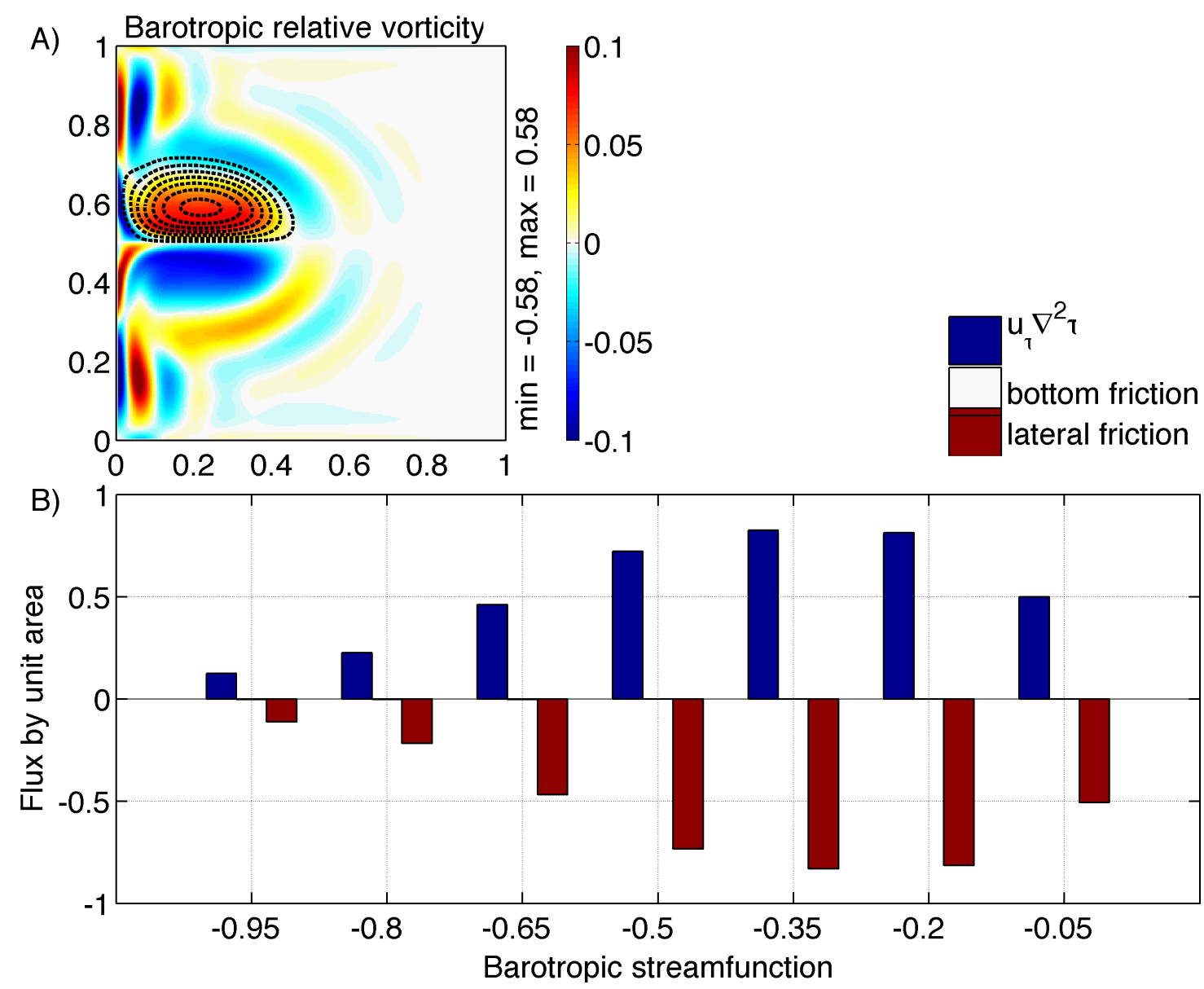

Figure 4-2: Barotropic potential vorticity budget for the case with $\tilde{\delta}_{I}=34.2 \mathrm{~km}$ $\left(h_{0}=120 \mathrm{~m}\right)$ integrated over a series of regions enclosed by barotropic streamlines. Panel A), barotropic relative vorticity field scaled with the maximum planetary vorticity. Overlaid are contours of the barotropic streamfunction delimiting the regions of integration, $\frac{\phi}{|\min \phi|} \in[-0.95,-0.05]$. Panel B), integral of the vorticity flux divergence by unit area for the different regions. All terms for the integrated budget are as indicated in Eq.(4.5). The bottom friction contribution is very small in all integrals.

layer. In the linear case corresponding to the weakest forcing, the maximum baroclinic relative vorticity and the maximum baroclinic streamfunction are both situated at the central latitude of the northern gyre. The baroclinic-baroclinic advective flux is then such that it drives a weak, order $\delta_{I}^{2}$, barotropic circulation in the boundary layer. The circulation is antisymmetric with respect to the central latitude of the northern gyre and consists of a clockwise cell in the northwest corner and an anti-clockwise cell toward the mid-latitude. 

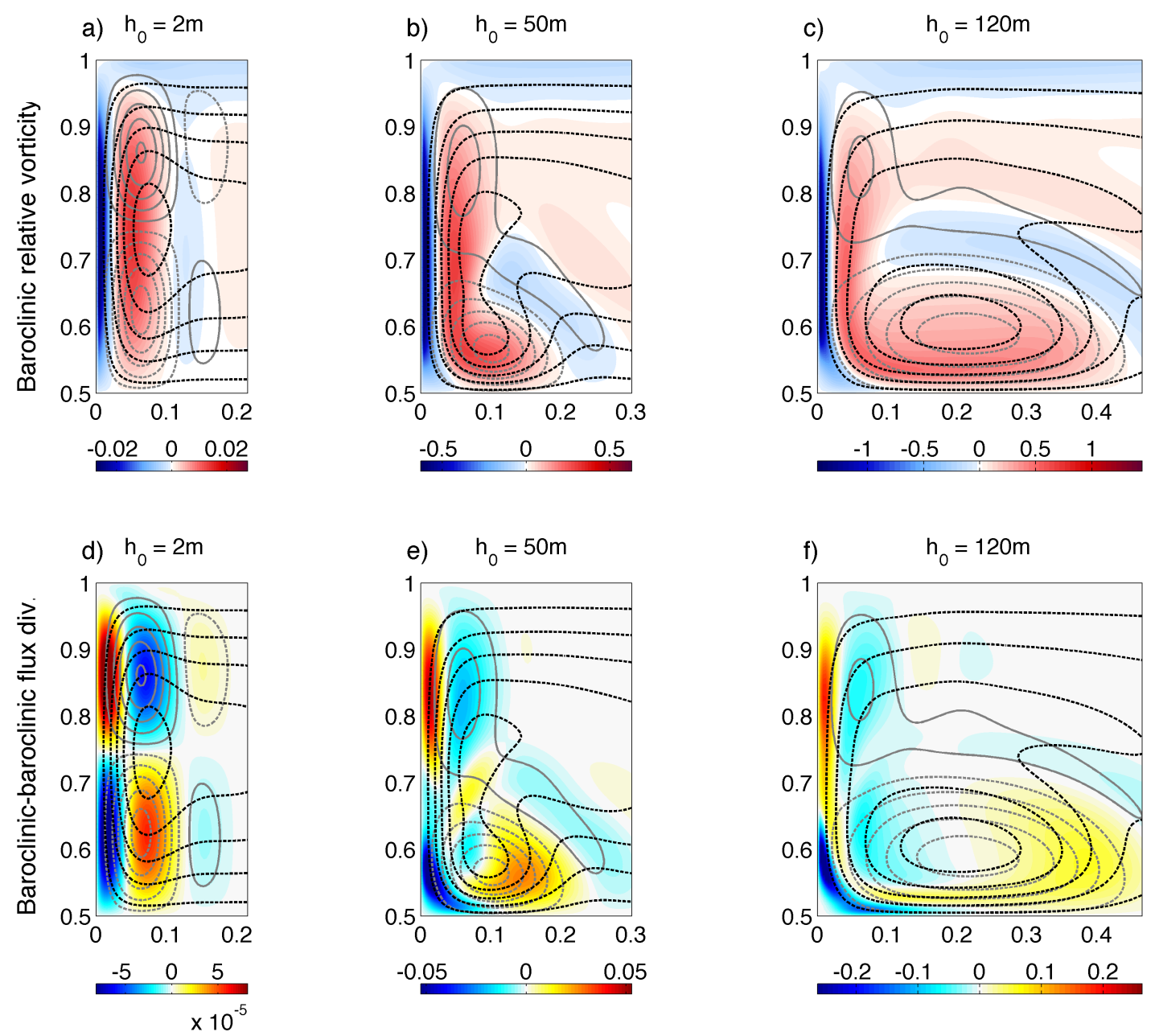

Figure 4-3: Barotropic vorticity budget in the western boundary layer and recirculation region in the northern half-basin for the case with $h_{0}=2,50$ and $120 \mathrm{~m}$. Panels a-b-c, baroclinic relative vorticity scaled with the maximum planetary vorticity. Panels d-e-f, baroclinic-baroclinic vorticity flux divergence (sign as in Eq.(4.4)). Overlaid are select streamlines for the baroclinic flow in black, and the barotropic flow in gray.

When the ocean is forced stronger and $\delta_{I}$ increases, the circulation looses its symmetry properties. Because the advection of relative vorticity in the boundary layer becomes significant, the region of large positive relative vorticity and the maximum of the streamfunction both shift toward the mid-latitude. A baroclinic recirculation gyre filled with positive baroclinic relative vorticity forms. Consequently, the spatial distribution of the divergence of the baroclinic-baroclinic advective flux, which is the driving force for the barotropic flow, changes. Nevertheless, the same two cell 
barotropic circulation as in the linear limit is generated with the one big difference that for larger $\delta_{I}$ the anti-clockwise cell situated toward the mid-latitude is much larger and stronger than the clockwise cell in the northwest corner. Thus, the existence of the barotropic recirculation gyres is directly related to the formation of baroclinic recirculation gyres.

\subsection{Dynamics of the baroclinic circulation}

Similarly to the barotropic vorticity balance, the steady, non-linear vorticity equation for the baroclinic vertical mode can be written in a flux divergence form as,

$$
\begin{aligned}
& \nabla \cdot[\underbrace{\tau \hat{\mathbf{x}}}_{\text {planetary }}+\underbrace{\delta_{I}^{2} \mathbf{u}_{\phi}\left(\nabla^{2} \tau-F \tau\right)+\delta_{I}^{2} \mathbf{u}_{\tau} \nabla^{2} \phi}_{\text {barotropic-baroclinic }}+\underbrace{\delta_{I}^{2}(1-2 \delta) \mathbf{u}_{\tau} \nabla^{2} \tau}_{\text {baroclinic-baroclinic }} \\
& -\underbrace{\delta_{M}^{3} \nabla\left(\nabla^{2} \tau\right)-\delta_{S} \nabla(\phi-\delta \tau)}_{\text {frictional }}]=\underbrace{\frac{\tau+U_{T} F_{T}(y)}{\delta_{T}}}_{\text {thermal forcing } \equiv-F w_{*}} .
\end{aligned}
$$

The main difference from the barotropic equation is the presence of the thermal relaxation forcing that acts as an external source of baroclinic vorticity. Another difference is the form of the advective terms. In the baroclinic balance, "mixed" terms are present that describe the advection of the baroclinic potential vorticity $q_{\tau}=\nabla^{2} \tau-F \tau$ by the barotropic velocity field $\mathbf{u}_{\phi}$, and the advection of the barotropic potential vorticity $q_{\phi}=\nabla^{2} \phi$ by the baroclinic velocity field $\mathbf{u}_{\tau}$. Both these terms are called barotropic-baroclinic vorticity flux. There is also a contribution from the baroclinic-baroclinic vorticity flux, $\mathbf{u}_{\tau} q_{\tau}$. This term is non-zero only if $\delta \neq 1 / 2$, i.e. when the two density layers are of different depths.

When integrated over a region $C_{\tau}$ enclosed by a baroclinic streamline, the vorticity equation (4.6) leads to to the following budget for the baroclinic vorticity

$$
0=-\oint_{\partial C_{\tau}}\left[\delta_{I}^{2} \mathbf{u}_{\phi} \nabla^{2} \tau-\delta_{M}^{3} \nabla\left(\nabla^{2} \tau\right)-\delta_{S}(1-\delta) \nabla(\phi-\delta \tau)\right] \cdot \hat{\mathbf{n}} d l+\iint_{C_{\tau}} \frac{\tau+U_{T} F_{T}(y)}{\delta_{T}} d A
$$


Use was made of the fact that all advective fluxes by the baroclinic velocity do not contribute to the the cross-streamline flux. What the integrated budget shows is that the barotropic advection of baroclinic relative vorticity and the frictional fluxes in and out of the region have to balance the input of vorticity by the thermal relaxation forcing. Because the cross-isopycnal velocity that drives the circulation is not externally specified by geographical location but depends on the solution itself, the amount and even sign of vorticity input depend on the solution as well. This is different from the case of a wind-driven circulation, where the Ekman pumping velocity is prescribed.

The integrated baroclinic vorticity budget for the case with strongest thermal forcing featuring the largest recirculations, is shown in Figure 4-4. The integration is done over a series of regions bounded by baroclinic streamlines encompassing progressively larger portions of the northern gyre. The vorticity fluxes resulting from advection, lateral diffusion and thermal forcing as described in Eq.(4.7), but divided in addition by the area of the region of integration, are plotted. The bottom frictional flux has been omitted since it was found that it is several orders of magnitude smaller than any of the other fluxes. The same analysis, except for some sign reversals, holds for the southern gyre.

The interior of the northern gyre is characterized with positive baroclinic potential vorticity $q_{\tau}=\nabla^{2} \tau-F \tau$, with a thin band of negative relative vorticity present next to the walls because of the no-slip boundary condition (Figure 4-4(A)). The positive interior baroclinic vorticity is dominated by the positive vortex stretching component due to the cooling of the water column. From the vorticity budget integrated over the largest region containing nearly the entire northern half-basin, it can be seen that the thermal relaxation forcing is indeed the source of positive vorticity for the northern gyre. However, as progressively smaller in area regions are considered, the flux by unit area due to the thermal forcing decreases, and even changes sign in the center of the recirculation. Thus, although in general the northern half-basin is cooled with the thermal forcing acting as a source of positive vorticity, the center of the recirculation is actually warmed and negative vorticity is put into the system. 

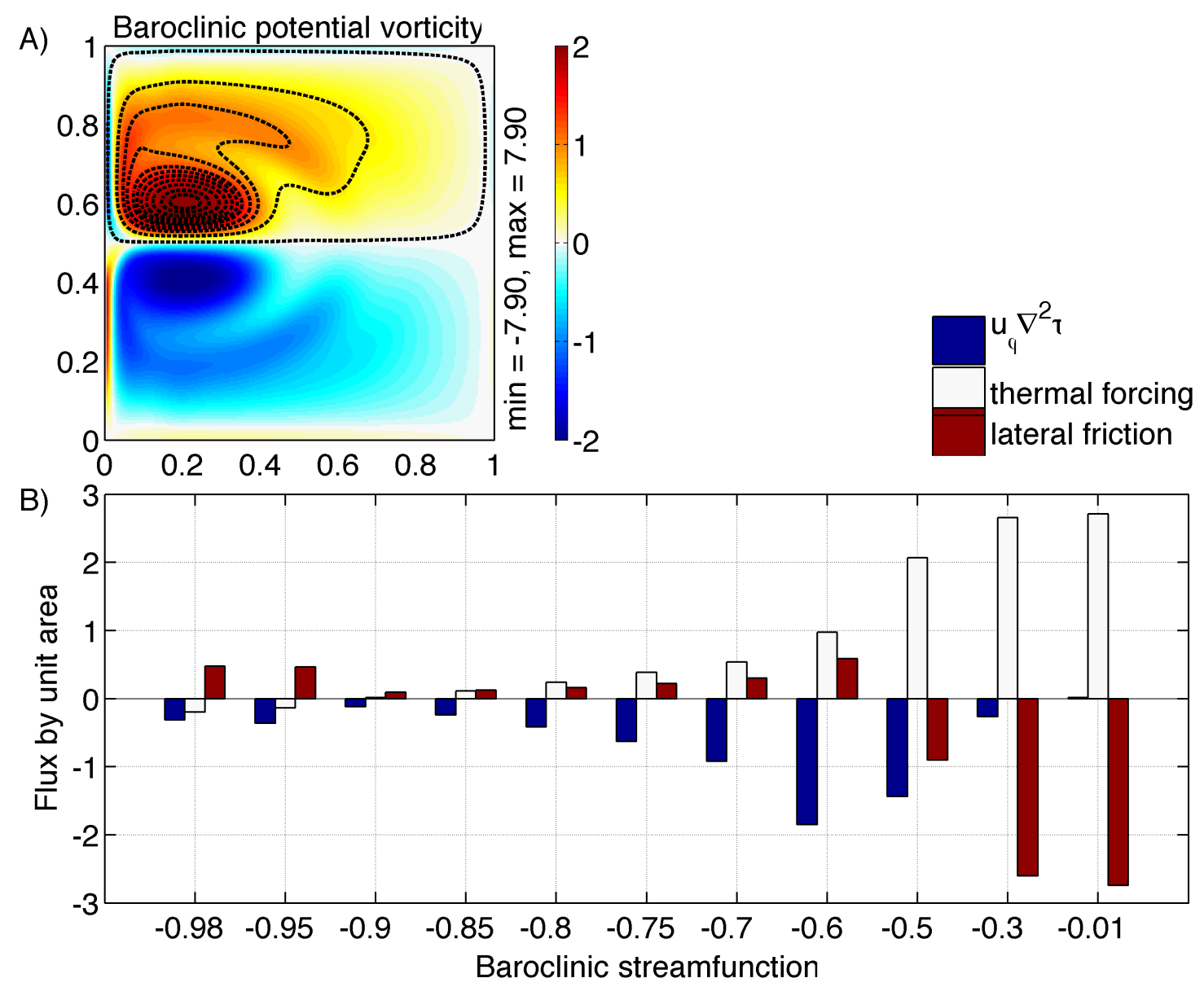

Figure 4-4: Baroclinic potential vorticity budget for the case with $\tilde{\delta}_{I}=34.2 \mathrm{~km}\left(h_{0}=\right.$ $120 \mathrm{~m}$ ) integrated over a series of regions enclosed by baroclinic streamlines. Panel A), baroclinic potential vorticity field (relative plus vortex stretching) scaled with the maximum planetary vorticity. Overlaid are contours of the baroclinic streamfunction delimiting the regions of integration, $\frac{\tau}{|\min \tau|} \in[-0.98,-0.01]$. Panel B), integral of the vorticity flux divergence by unit area for the different regions. All terms for the integrated budget are as indicated in Eq.(4.7). The bottom friction contribution has been omitted since it is negligible.

The barotropic-baroclinic advective flux $\mathbf{u}_{\phi} \nabla^{2} \tau$ across all interior streamlines is negative. This means that positive baroclinic vorticity is carried out of the regions by the barotropic flow and is eventually deposited next to walls where it is dissipated by lateral friction. For the outer most streamline containing nearly the entire northern half-basin, the barotropic-baroclinic flux is close to zero. This is partially due to the no-slip and no-normal flow conditions at the walls, but it indicates also that there is 

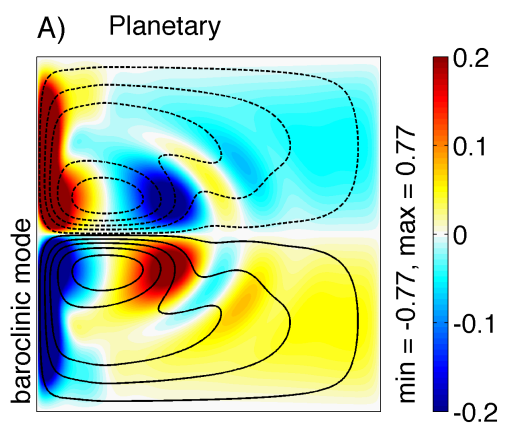

B) Barotropic-baroclinic

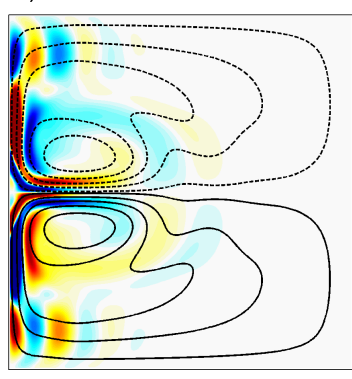

E) Lateral friction
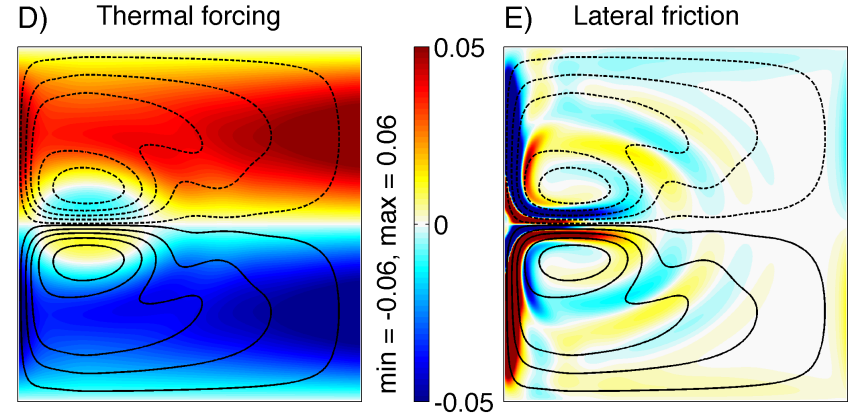

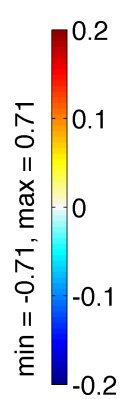

C) Baroclinic-baroclinic
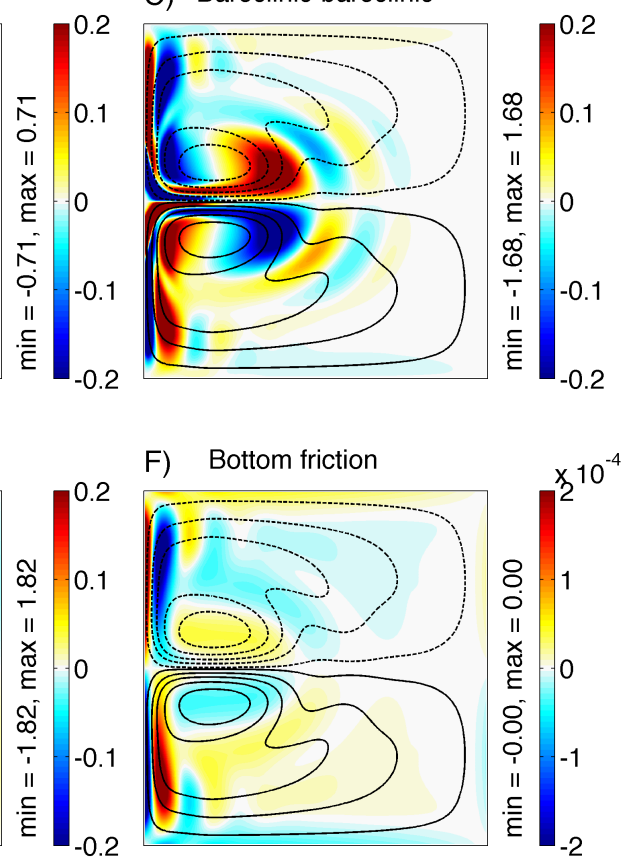

Figure 4-5: Baroclinic vorticity budget for the case with $\tilde{\delta}_{I}=35.6 \mathrm{~km}\left(h_{0}=120 \mathrm{~m}\right)$. All vorticity flux divergences are plotted with a sign convention as in Eq.(4.6). Selected baroclinic streamlines are overlaid on top for reference.

no advective inter-gyre flux of baroclinic vorticity.

Finally, concerning the lateral friction, its role for the half-basin as a whole is to flux out the vorticity put into the system by the thermal forcing. Indeed, the vorticity budget integrated over the largest region shows a balance between the input of positive baroclinic vorticity by the thermal forcing and its dissipation by lateral friction. For most of the interior regions however, the lateral frictional flux is actually positive, meaning that baroclinic vorticity is carried into the regions in amounts similar to those deposited by the thermal forcing. In the center of the recirculation, the lateral diffusion is the only positive term.

In Figure 4-5 the spatial distribution of the different baroclinic vorticity flux divergences as described in Eq.(4.6) is shown. The imbalance, not plotted, is on the order of $10^{-7}$ (small compared to 1 , the order of the largest flux divergence). In the eastern part of the basin the linear balance between input of vorticity by the thermal forcing 
and advection of planetary vorticity by the baroclinic flow holds. In the middle part of the basin and in the western boundary layer it is rather a balance between the advection of planetary vorticity, the baroclinic-baroclinic flux and the lateral diffusion of vorticity, with the barotropic-baroclinic advective flux important in the western boundary layer as well. In these regions the thermal forcing plays only a secondary role, while the bottom friction contribution is in general weak over the whole basin. Note that although the planetary advection and the baroclinic-baroclinic terms are very significant locally, they do not participate in the cross-streamline vorticity flux in the integrated vorticity budget. Finally, it can be seen that indeed the thermal relaxation forcing changes sign around the region of the recirculation gyres, leading to weak warming within the northern recirculation and weak cooling within the southern recirculation.

\subsection{Heat budget of the circulation}

The interface displacement $\eta$, which in nondimensional units is just equal to the opposite of the baroclinic streamfunction $-\tau$, represents also the vertically averaged temperature field. Hence, the equation for the evolution of the interface displacement can be viewed as the heat budget of the system. In nondimensional form this equation is*

$$
\begin{aligned}
\frac{\partial \eta}{\partial t}+\delta_{I}^{2} J(\phi, \eta) & =w_{i}-w_{*}+\frac{1}{\operatorname{Pr}} \delta_{M}^{3} \nabla^{2} \eta \\
& =w_{i}-\frac{1}{F \delta_{T}}\left(\eta-U_{T} F_{T}(y)\right)+\frac{1}{P r} \delta_{M}^{3} \nabla^{2} \eta
\end{aligned}
$$

What the heat budget expresses is that the rate of change of temperature following a fluid parcel advected by the barotropic flow is due to the vertical advection and mixing, the last one being represented through the cross-isopycnal flux resulting in water exchange between the density layers. In general, there is also a contribution

${ }^{*}$ For reference, the interface displacement $\eta$ is scaled with the vertical scale $D=U L f_{0} / g^{\prime}$, while the vertical and cross-isopycnal velocities $w_{i}$ and $w_{*}$ with $D /\left(\beta_{0} L\right)^{-1}$. 
from the lateral diffusion of heat by the unresolved eddy processes. However, for all calculations here we have set the Prandtl number $\operatorname{Pr}=\nu / \kappa$ to infinity. Equation (4.8) was previously used to define the vertical velocity $w_{i}$ at the interface between the two layers when the mass conservation condition (3.29) was discussed. Describing the heat budget of the system is therefore equivalent to describing the spatial structure of the resulting vertical velocity $w_{i}$. For a steady circulation, the vertical velocity is simply equal to the cross-isopycnal flux $w_{*}$ plus a contribution from the lateral advection of interface $\delta_{I}^{2} J(\phi, \eta)$.

\subsubsection{The cross-isopycnal flux}

One of the advantages of using a large-scale thermal forcing parameterized as relaxation of the interface is that it leads to a cross-isopycnal flux determined from internal dynamics, and not externally prescribed. The applied thermal forcing was chosen such that at least initially, when the ocean is at rest, the northern half-basin is cooled while the southern half-basin is warmed. However, because the cross-isopycnal flux depends on the solutions itself, this does not signify that in the final steady equilibrium reached by the system the sign of the cross-isopycnal flux remains the same as specified initially.

In Figure 4-6 the cross-isopycnal flux $w_{*}=\left(\eta-U_{T} F_{T}\right) / F \delta_{T}$ is plotted for the series of three steady calculations with progressively larger inertial boundary layer thickness. In the linear limit, the entire northern/southern half-basin is cooled/warmed. For the stronger forced cases however regions with opposite signed cross-isopycnal velocity form in each gyre. They are situated approximatively within the baroclinic recirculations, although not perfectly aligned with them (see Figure 4-5(D) for $\tilde{\delta}_{I}=34.2 \mathrm{~km}$ ). Next, we will demonstrate that the location where the thermal forcing changes sign can be identified if the baroclinic vorticity equation is examined using the method of the characteristics.

Suppose that the barotropic circulation $\phi$ is known. Then, neglecting the advection of relative vorticity, which is a reasonable simplification for the interior large-scale 
ocean circulation, the baroclinic vorticity equation (3.26) reduces to

$$
J(\tau, \underbrace{y+\delta_{I}^{2} F \phi}_{\hat{\phi}(x, y)})=U_{w} F_{w}(y)+\frac{1}{\delta_{T}}\left(\tau+U_{T} F_{T}(y)\right)+\text { diss. }
$$

The contours of the function $\hat{\phi}$ are the characteristics of the partial differential equation (4.9), also called geostrophic contours (Rhines and Young, 1982). They represent the curves along which information about the blocking action of the eastern wall is propagated westward in the basin by the long internal Rossby waves. The expression for the geostrophic contours $\hat{\phi}$, can be rewritten also as

$$
\hat{\phi}=y+\delta_{I}^{2} F \phi=y+\Omega \phi
$$

where $\Omega=U / \beta_{0} R_{d}^{2}$ is the inverse of the nondimensionalized long internal Rossby wave speed. The form of the geostrophic contours is set thus by the barotropic circulation. Strong enough eastward barotropic flow can arrest the westward propagating Rossby waves and create regions of geostrophic contours isolated from the eastern wall.

In the case of a wind-only driven ocean, the barotropic circulation, and hence the geostrophic contours, are determined from the wind stress and can be easily computed using Sverdrup balance. Because the potential vorticity of the lower, not-directly forced layer is conserved, the baroclinic streamfunction determined from Eq.(4.9) is such that it cancels the barotropic transport and adds up to no motion in the lower layer on blocked geostrophic contours (Rhines and Young, 1982; Spydell and Cessi, 2003). If on the other hand a cross-isopycnal flux is included, then the potential vorticity conservation constraint is removed and motion in the lower layer on blocked geostrophic contours is allowed. Luyten and Stommel (1986) describe this situation as circulation "steered by wind and driven by buoyancy".

For a thermally-only forced ocean, when the advection of relative vorticity is neglected all together, there is no barotropic circulation. Consequently, the geostrophic contours coincide with latitude lines. However, we have seen that because of the non- 
linear coupling between the baroclinic and barotropic flow a weak, but not insignificant, barotropic circulation can be generated. We can thus define the geostrophic contours taking into account the barotropic flow driven by the advection of baroclinic relative vorticity by the baroclinic flow, while neglecting again all nonlinear terms involving the relative vorticity in the baroclinic, directly forced component of the circulation.

In Figure 4-6 the geostrophic contours for the steady, thermally-forced circulation determined in such a way are overlaid on top of the corresponding cross-isopycnal velocity $w_{*}$. For the most weakly forced case, the geostrophic contours are basically latitude lines. There are no recirculation gyres and no sign reversal of the crossisopycnal flux. For the most strongly forced case however, the barotropic circulation driven by the nonlinear coupling is strong enough, so that it is able to arrest the westward propagation of Rossby waves. Regions with closed geostrophic contours isolated from the eastern wall form. The limits of these regions match very closely the location of the opposite signed cross-isopycnal velocity in each half-basin.

Therefore, although for a thermally-only forced ocean, the barotropic circulation is determined from nonlinear dynamics and needs to be computed numerically, the geostrophic contours, depending on their geometry (blocked or isolated), separate flow with different behavior, similarly to what is occurring in the wind-driven case.

\subsubsection{The vertical velocity}

The vertical velocity $w_{i}$ at the interface between the two density layers and its decomposition into local vertical motion of the interface and cross-isopycnal flux are shown in Figure 4-7 for the series of three steady calculations with progressively larger inertial boundary layer thickness.

The cross-isopycnal flux $w_{*}$ represents the part of the vertical velocity that is associated with density transformation as the fluid crosses between the two isopycnal layers due to the presence of sources of heating and cooling. In the previous section we discussed how the geometry of the geostrophic contours can affect the spatial 
A) Cross-isopycnal $w_{*}, h_{0}=2 m$

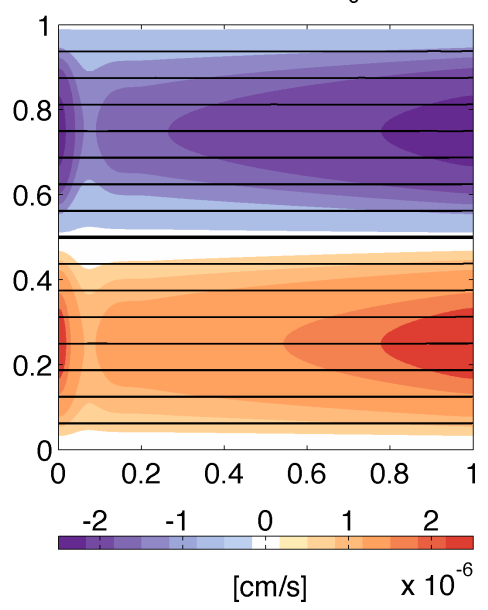

B) Cross-isopycnal $w_{*}, h_{0}=50 m$

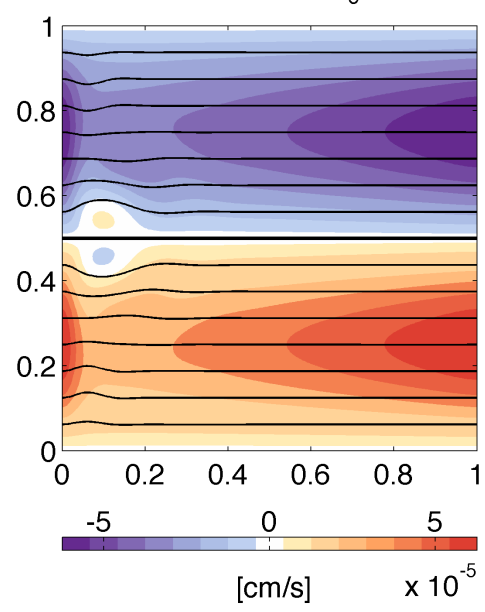

C) Cross-isopycnal $w_{*}, h_{0}=120 \mathrm{~m}$

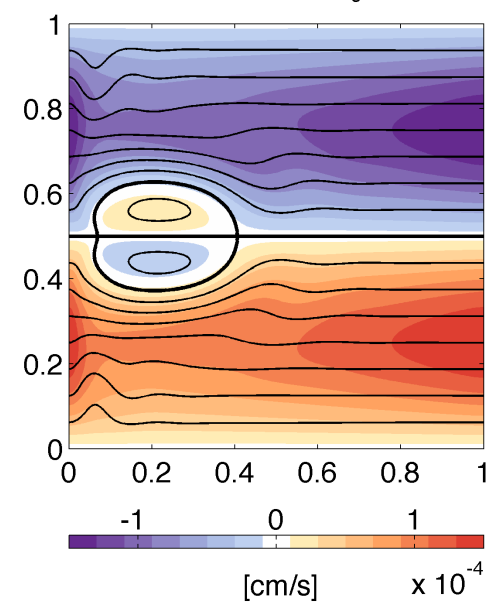

Figure 4-6: Cross-isopycnal flux $w_{*}=\left(\eta-U_{T} F_{T}(y)\right) / F \delta_{T}$ (in color) and geostrophic contours $\hat{\phi}=y+\Omega \phi$ with $\Omega=U / \beta_{0} R_{d}^{2}$ (in black lines) for the steady regime thermallyforced circulation with $\tilde{\delta}_{I}=4.5,22.1$ and $34.2 \mathrm{~km}\left(h_{0}=2,50\right.$ and $\left.120 \mathrm{~m}\right)$.

distribution of heating and cooling over the basin. In addition, we can note that the stronger is the thermal forcing, the larger is the magnitude of the cross-isopycnal velocity. At a given latitude, the largest cooling/warming occurs at the walls. Because the interface displacement is constant along the basin boundary, and since we choose a zonally uniform target height $F_{T}(y)$, the departure between the two at the wall depends only on latitude. Consequently, the cross-isopycnal velocity $w_{*}$ takes the same value on the eastern and western side of the basin, at a given latitude.

The vertical velocity $w_{i}$ has also an adiabatic component due to the local vertical motion of the interface, where no density transformation is involved. In the steady regime, the rate of change of the interface is given by the horizontal advection of interface by the barotropic flow. This term is largest in the regions where the interface slopes are the strongest, i.e the western boundary layer and the recirculations. When the inertial boundary layer thickness is increased, so does the magnitude of the local vertical motion of the interface.

In the linear limit when the nonlinear advective terms are negligible, the vertical velocity $w_{i}$ is dominated by the cross-isopycnal flux $w_{*}$. However, as the circulation becomes more nonlinear the vertical velocity $w_{i}$ becomes strongly affected by the 
A) $\quad \tilde{\delta}_{I}=4.5 \mathrm{~km}$
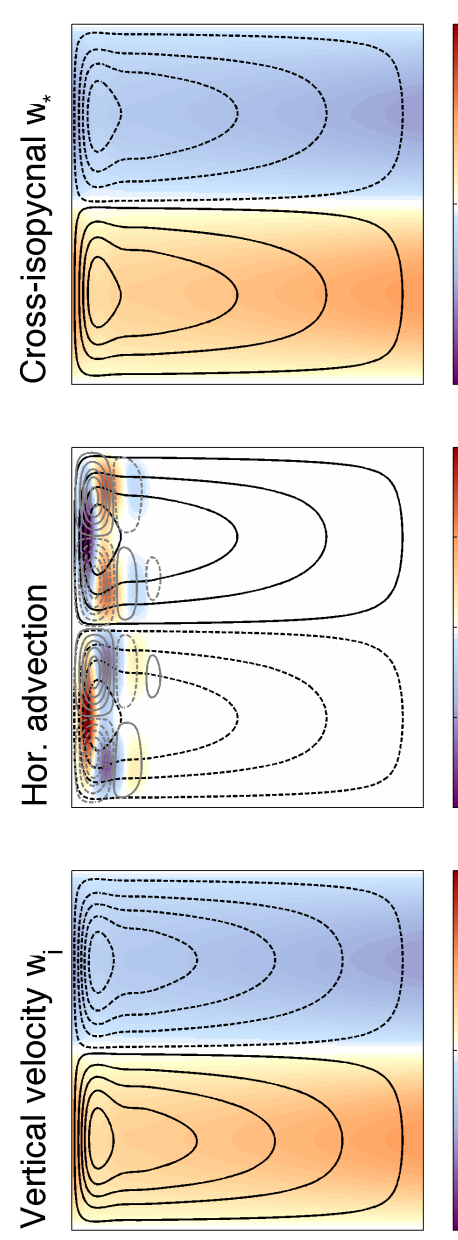

B) $\quad \tilde{\delta}_{I}=22.1 \mathrm{~km}$

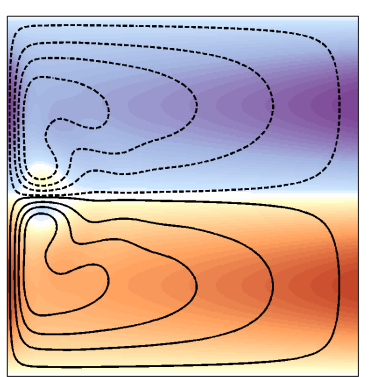

-0.05
$\times 10^{-4}$
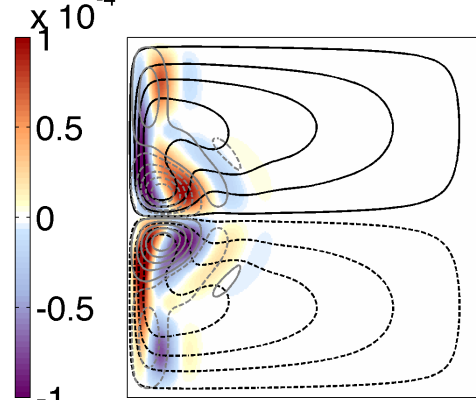

0.05

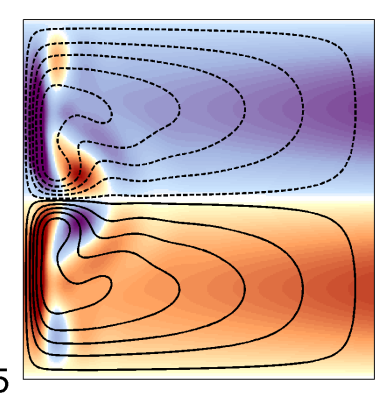

C) $\quad \tilde{\delta}_{I}=34.2 \mathrm{~km}$
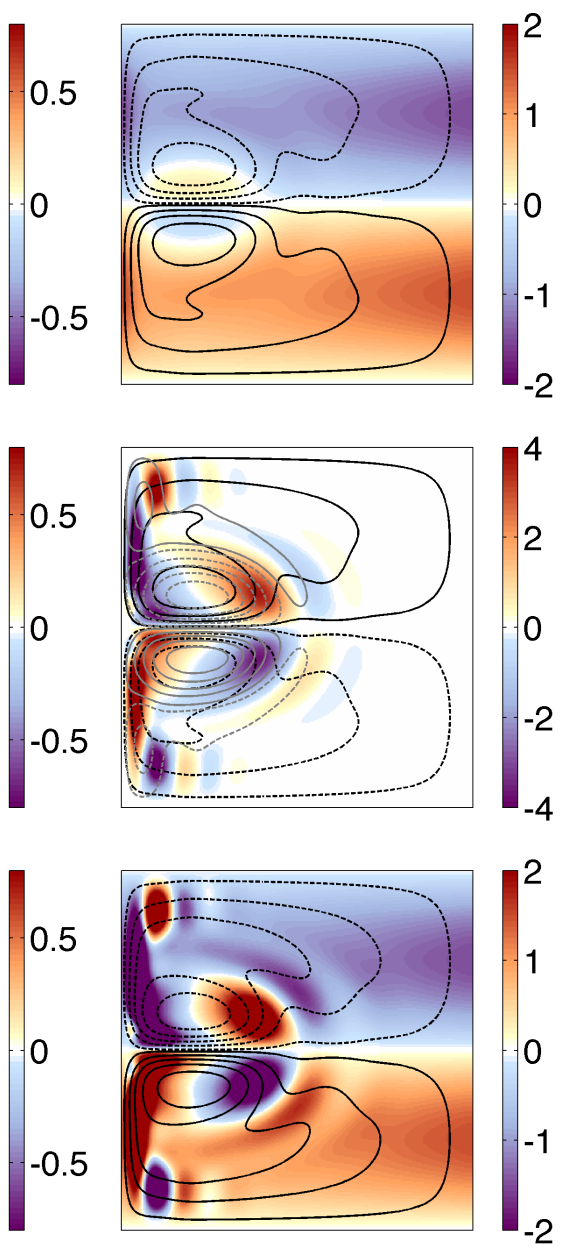

Figure 4-7: Vertical velocity for the steady regime thermally-forced circulation for the case A), $\left.\tilde{\delta}_{I}=4.5 \mathrm{~km}, \mathrm{~B}\right), \tilde{\delta}_{I}=22.1 \mathrm{~km}$, and C), $\tilde{\delta}_{I}=34.2 \mathrm{~km}$. First row: the crossisopycnal velocity $w_{*}=\left(\eta-U_{T} F_{T}\right) / F \delta_{T}$. Selected baroclinic streamlines are overlaid in black. Second row: the horizontal advection contribution $\delta_{I}^{2} \mathbf{u}_{\phi} \cdot \nabla \eta$. Overlaid are selected barotropic streamlines in gray, and contours of $\eta$ in black. Third row: the net vertical velocity at the interface $w_{i}=w_{*}+\delta_{I}^{2} \mathbf{u}_{\phi} \cdot \nabla \eta$. Selected baroclinic streamlines are overlaid in black. All velocities are in $10^{-4} \mathrm{cms}^{-1}$.

adiabatic component due to the horizontal advection of interface. In general, the northern half-basin is characterized with cooling and downward motion, while the southern half-basin with warming and upward motion. Patches of opposite signed vertical velocities are present in the recirculations and in the southwest and northwest basin corners where steep interface slopes are present. The strongest vertical velocities 
occur next to the western wall and are mostly due to the local vertical motion of the interface. Because of the mass conservation constraint, the spatial pattern for $w_{i}$ in all cases is such that it integrates to zero over the basin area.

\subsection{Summary}

The goal of this chapter was to examine the steady regime circulation when a thermal only forcing is applied to a 2-layer QG ocean. For all calculations analyzed in this chapter the Reynolds number was kept low, so that no instabilities occur and the circulation remains steady. The following major conclusions were drawn:

(1) Because the thermal relaxation forcing is applied at the interface between the two isopycnal layers, it projects only on the baroclinic vertical mode of the circulation. Consequently, in the linear limit a typical half-basin warming / half-basin cooling thermal forcing drives a purely baroclinic double-gyre flow (Pedlosky and Spall, 2005). In this sense the thermal forcing is different from the wind forcing, that induces both a baroclinic and a barotropic response.

(2) A vertically integrated circulation, that is not directly thermally-forced, can be generated in a thermally-only forced ocean through the nonlinear coupling between the barotropic and baroclinic flow. More specifically, the advection of baroclinic relative vorticity by the baroclinic flow acts as a source of barotropic vorticity driving thus a vertically integrated circulation. The barotropic circulation in the steady regime is generally weak, since its source is proportional to the inertial boundary layer thickness $\delta_{I}^{2}$. It is in the form of recirculation gyres that expand and strengthen when $\delta_{I}$ is increased.

(3) In the presence of thermal forcing, the vertical velocity at the interface between the two layers is composed of the local vertical motion of the interface and the cross-isopycnal flux. The cross-isopycnal flux, being parameterized as relaxation of the interface, is determined from internal dynamics. Its spatial distribution 
is affected by the geometry of the geostrophic contours, determined by the nonlinearly driven barotropic flow. In the linear limit the vertical velocity is dominated by the cross-isopycnal flux, but when $\delta_{I}$ is increased it is rather the lateral advection of interface that sets the regions of strongest vertical motions. 


\section{Chapter 5}

\section{Thermally-forced ocean in the time-dependent regime}

In this chapter the focus is on the time-mean circulation and variability of a strongly nonlinear thermally-forced ocean. It is found that the circulation is characterized by a large range of temporal and spatial scales of variability, including large-scale, fast, barotropic basin modes. It is demonstrated that the presence of strong basin modes plays a major role in shaping the circulation. They drive a time-mean basin-scale barotropic flow, not present in the steady regime. They are directly responsible for some of the baroclinic flow variability and interfere with the inertial recirculations. Although the particular case of a thermally-forced ocean is examined, all conclusions about the effect of the basin modes are relevant to any other systems that exhibit variability in the form of barotropic normal modes, no matter how they are excited. In other words, we are expecting that the same type of behavior can be found in a wind-driven ocean, if barotropic basin modes are excited. Examples of such systems are marginal seas and semi-enclosed by bathymetry basins such as the Mascarene basin (Warren et al., 2002) and the Argentine basin (Fu et al., 2001; Weijer et al., 2007a), where barotropic basin modes have been observed.

One question that will not be addressed here but in Chapter 6, is the origin of the basin mode variability in a thermally-forced ocean. 


\subsection{Model setup}

The configuration considered for the time-dependent calculations is slightly different from the one used for the steady regime calculations. The major similarities and differences between the two model setups are summarized next, with the full set of parameters used in the time-dependent calculations listed in Table 5.1.

As for the steady calculations, the ocean is driven by a thermal forcing that corresponds to heating in the southern half-basin and cooling in the northern halfbasin. The amplitude of the target height however is larger than in Chapter 4, which leads to a highly nonlinear regime of circulation, characterized with inertial boundary layer $\tilde{\delta}_{I}=70 \mathrm{~km}$ several times larger than the Munk boundary layer $\tilde{\delta}_{M}=13 \mathrm{~km}$.

The circulation is computed in larger basin with $L=3840 \mathrm{~km}$, so that it is closer in dimensions to a realistic ocean basin like the North Atlantic, for example. Similarly, a larger internal deformation radius $R_{d}=55 \mathrm{~km}$ is used. This leads to faster propagating internal Rossby waves with a transit time across the basin on the order of 2 years. Consequently, the relaxation timescale $\gamma$ has been adjusted, so that again we have a thermal boundary layer $\delta_{T}$ on the order of one, signifying that the internal Rossby waves are only weakly damped by the interface relaxation.

A small value for the eddy viscosity is applied in order to obtain a high Reynolds number regime, where at least part of the eddy processes are explicitly resolved by the model and need not to be parameterized. The Munk boundary layer width for the choice of eddy viscosity $\nu=40 \mathrm{~m}^{2} \mathrm{~s}^{-1}$ is on the order of the grid resolution $\left(\tilde{\delta}_{M}=13 \mathrm{~km}\right.$, while $\left.\Delta x=15 \mathrm{~km}\right)$. Thus, the frictional boundary layer is at best marginally resolved. This may have the consequence that the effective Reynolds number for the calculations is actually smaller than the one specified in Table 5.1, due to discretization errors leading to additional numerical diffusion.

A set of two different values for the bottom drag are used in order to illustrate some interesting features of the circulation. Note that although the two values differ by a factor of 100 , in both cases the bottom friction is too weak for the Stommel 


\begin{tabular}{|c|c|c|c|}
\hline \multicolumn{2}{|c|}{ Dimensional parameters } & \multicolumn{2}{|c|}{ "Nondimensional parameters } \\
\hline$L_{x}, L_{y}$ & $3840 \mathrm{~km}$ & $\delta$ & 0.17 \\
\hline$H_{1}$ & $500 \mathrm{~m}$ & $F$ & 4900 \\
\hline$H_{2}$ & $2500 \mathrm{~m}$ & $\tilde{\delta}_{M}\left(\delta_{M}\right)$ & $12.6 \mathrm{~km}(0.003)$ \\
\hline$f_{0}$ & $0.83 \times 10^{-4} s^{-1}$ & $\tilde{\delta}_{S}\left(\delta_{S}\right)$ & $0.05-5 \mathrm{~km}\left(10^{-5}-10^{-3}\right)$ \\
\hline$\beta_{0}$ & $2 \times 10^{-11} m^{-1} s^{-1}$ & $\tilde{\delta}_{T}\left(\delta_{T}\right)$ & $3805 \mathrm{~km}(0.99)$ \\
\hline$\rho_{0}$ & $1000 \mathrm{kgm}^{-3}$ & $\tilde{\delta}_{I}\left(\delta_{I}\right)$ & $70.9 \mathrm{~km}(0.02)$ \\
\hline$R_{d}$ & $55 \mathrm{~km}$ & $\operatorname{Re}$ & 9640 \\
\hline$g^{\prime}$ & $0.05 \mathrm{~ms}^{-2}$ & & \\
\hline$\nu$ & $40 m^{2} s^{-1}$ & & \\
\hline$r$ & $10^{-9}-10^{-7} s^{-1}$ & Grid anc & olution \\
\hline$\gamma$ & 1.99 years & grid & $257 \times 257$ \\
\hline$h_{0}$ & $640 \mathrm{~m}$ & $\Delta x$ & $15 \mathrm{~km}$ \\
\hline
\end{tabular}

Table 5.1: Values of the parameters used in the time-dependent calculations. The left column defines the dimensional parameters. The right column gives the values of the nondimensional parameters and the grid resolution.

boundary layer to be resolved $\left(\tilde{\delta}_{S}=0.05-5 \mathrm{~km}\right.$, while $\left.\Delta x=15 \mathrm{~km}\right)$.

All simulations are started from an ocean at rest and integrated initially for 40-50 years - a time interval large compared to the restoring timescale, so that the system reaches statistical equilibrium. After the circulation is established, the system is integrated for an additional number of years and the output saved, so that different analyses can be carried out.

\subsection{Overview of the circulation}

In this section an initial overview diagnosing different features of the time-mean circulation and its variability is presented.

\subsubsection{Time-mean circulation}

The barotropic and baroclinic components of circulation averaged in time over a period of 120 years are shown in Figure 5-1. As already mentioned, two values for the 
Weak bottom drag
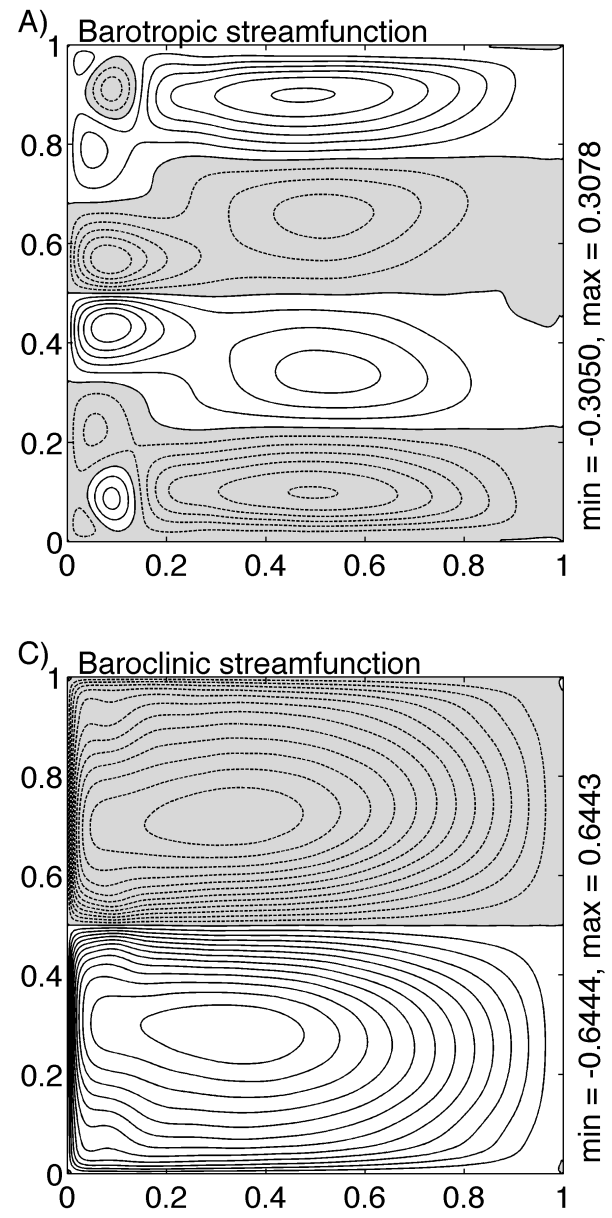

Strong bottom drag
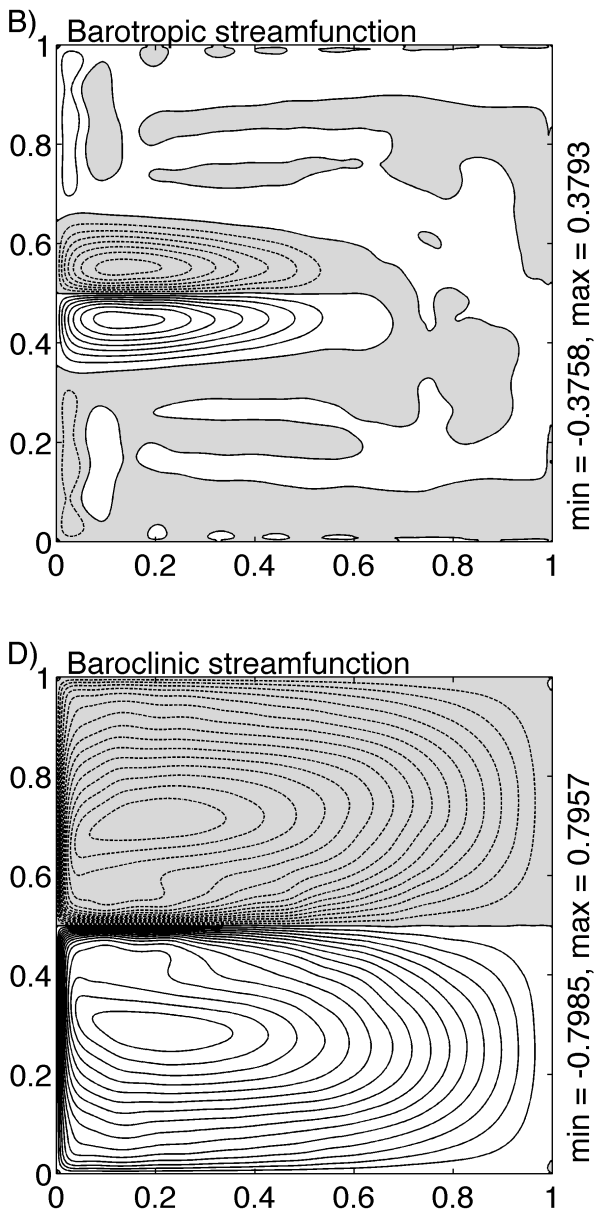

Figure 5-1: Time-mean circulation for: A) and C), the weak bottom friction case with $r=10^{-9} s^{-1}$; B) and D), the strong bottom friction case with $r=10^{-7} s^{-1}$. The top row is the time-mean barotropic streamfunction, the bottom row, the timemean baroclinic streamfunction. The streamfunction is scaled by the maximum linear baroclinic streamfunction $\tau^{(\text {lin })}=\frac{g^{\prime} h_{0}}{f_{0}}\left(1-e^{-\frac{1}{\delta_{T}}}\right)=0.25 \times 10^{6} m^{2} s^{-1}$. The contour interval on all plots is 0.05 . Negative values are shaded.

bottom drag differing by a factor of 100 are used. For the weak bottom friction case, $r$ is equal to $10^{-9} s^{-1}$, which corresponds to a frictional spin-down time $1 / r \approx 32$ years or a Stommel boundary layer width $\tilde{\delta}_{S}=0.05 \mathrm{~km}$. For the strong bottom friction case, $r$ is equal to $10^{-7} s^{-1}$, with a frictional spin-down time $1 / r \approx 120$ days or $\tilde{\delta}_{S}=5 \mathrm{~km}$. Although in both cases the Stommel frictional layer is not resolved by 
the grid resolution, there are some significant differences in the resulting circulation.

The most striking difference is that for the weak bottom friction case there is a substantial time-mean barotropic circulation. It consists of a 4-gyre basin-scale flow, plus in addition recirculations next to the western boundary. There is no such 4-gyre flow in the steady regime nor when stronger bottom friction is applied. This indicates that 1) the mechanism responsible for driving the time-mean barotropic circulation relies on the time-dependence of the flow, and that 2) it is very sensitive to the level of bottom friction in the system.

The size and strength of the inertial recirculations is also sensitive to the applied bottom drag. It can be seen that the recirculations extend much further east into the domain and are slightly stronger in the large bottom friction case. The fact that the circulation is more vigorous when the bottom drag is larger is counterintuitive, given that bottom friction is supposed to damp the barotropic flow.

The time-mean baroclinic circulation is sensitive to the magnitude of the bottom drag as well. The amplitude of the baroclinic streamfunction is greater for the larger bottom friction case. This could be rationalized given that by definition the bottom friction damps selectively the lower layer circulation only. Thus, applying a stronger bottom drag should make the flow more baroclinic.

The weak and strong bottom friction baroclinic circulations differ not only by their maximum streamfunction, but also by the east-west structure of the flow. The strong bottom friction circulation is western intensified and has a well defined free jet at mid-latitude, that penetrates zonally almost half-way into the basin. The weak bottom friction circulation on the other hand seems more inertially dominated - it is less western intensified, with the minimum/maximum of the streamfunction occurring almost one third east into the basin, and is characterized with a lack of well defined free jet in the time-mean. There are no recirculation in the time-mean baroclinic flow in either case. 


\section{Weak bottom drag}

Strong bottom drag
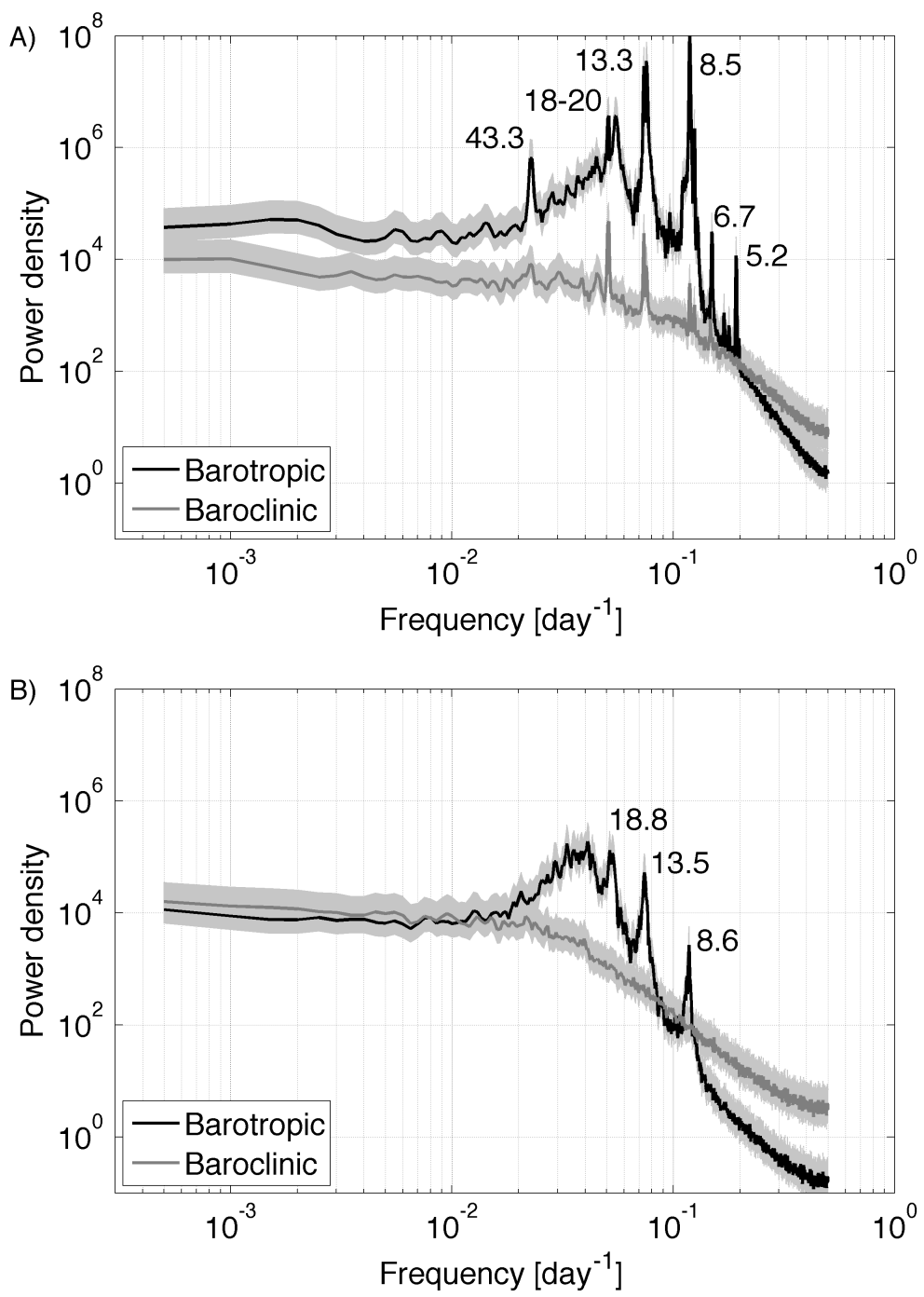

Figure 5-2: Power density spectrum with a 95\% confidence interval of the instantaneous barotropic and baroclinic streamfunctions for: A), the weak bottom friction case with $r=10^{-9} s^{-1}$; B), the strong bottom friction case with $r=10^{-7} s^{-1}$. The period, in days, for the largest spectral peaks is indicated.

\subsubsection{Temporal variability}

The magnitude of bottom friction applied to the system affects not only the timemean circulation, but also the variability of the flow.

A spatial average over the basin area is a convenient way to define a measure of the instantaneous circulation. However, because of the constraint of mass conservation and because of the high degree of anti-symmetry of the flow with respect to the 
midlatitude, such basin average is subject to a high degree of cancellation. Instead, as a measure of the instantaneous circulation the instantaneous streamfunction averaged over 100 randomly chosen grid points within the domain is taken (for all power density plots the same 100 points are considered).

Time series of the instantaneous barotropic and baroclinic streamfunctions are collected for a time interval of 27 years, subsampled every day. The power density spectra of the barotropic and baroclinic streamfunction are plotted in Figure 5-2 as function of the frequency expressed in days, for both the weak and strong bottom friction cases. By definition, the area under the power spectrum is representative for the variance of the corresponding time series. Comparing the weak and strong bottom friction cases shows that, as it could be anticipated, both the barotropic and baroclinic variability of the system, are damped by the bottom friction.

In addition to making the flow more laminar, the bottom friction also affects the nature of its variability. In the weak bottom friction case, the barotropic flow spectrum is dominated by distinct high-frequencies peaks, with periods ranging from several days to over a month. The baroclinic flow variability is weaker than that of the barotropic flow, as indicated by the lower laying power spectrum. Distinct peaks at periods closely matching those of the barotropic flow are present, implying a direct relationship between the barotropic and baroclinic flow variability. In the strong bottom friction case on the other hand, the high-frequency peaks in the barotropic spectrum are still present but with much smaller amplitude, while they are completely absent from the baroclinic spectrum.

In order to help identify the process leading to the observed high-frequency spectral peaks for the barotropic streamfunction, a statistical tool, the Hilbert empirical orthogonal functions (HEOF), is used. The HEOF analysis is a variation of the empirical orthogonal function (EOF) analysis, which is used to describe the spatial and temporal variability of a gridded data series in terms of orthogonal functions or statistical modes (von Storch and Zwiers, 1999). The technique is normally considered successful when most of the variance of the data field can be explained by the first 
few orthogonal functions, which spatial patterns need then to be related to possible dynamical mechanisms. The EOF analysis strength is in identifying standing oscillations, while the HEOF analysis is a modification that makes it appropriate for detecting propagating oscillations. A brief overview of the basic principles of the HEOF analysis technique is given in Appendix C.

The HEOF analysis is done using a 20-year calculation, where the instantaneous barotropic streamfunction is subsampled every 2 days on a coarse $65 \times 65$ grid, giving a resolution of $\Delta x=60 \mathrm{~km}$. The subsampling is done in order to limit the size of the covariance matrix used in the analysis. The first 10 eigenvectors, corresponding to the 10 largest eigenvalues in decreasing order, are then computed, using the Matlab singular value decomposition function. It has been verified a posteriori that sufficient time and spatial resolution has been kept in order to resolve the processes of interest.

The eigenvalues of the covariance matrix, expressed in terms of percentage of explained total variance are shown in Figure 5-3, for the weak and strong bottom friction cases. For the weak bottom friction case, the first mode explains close to $70 \%$

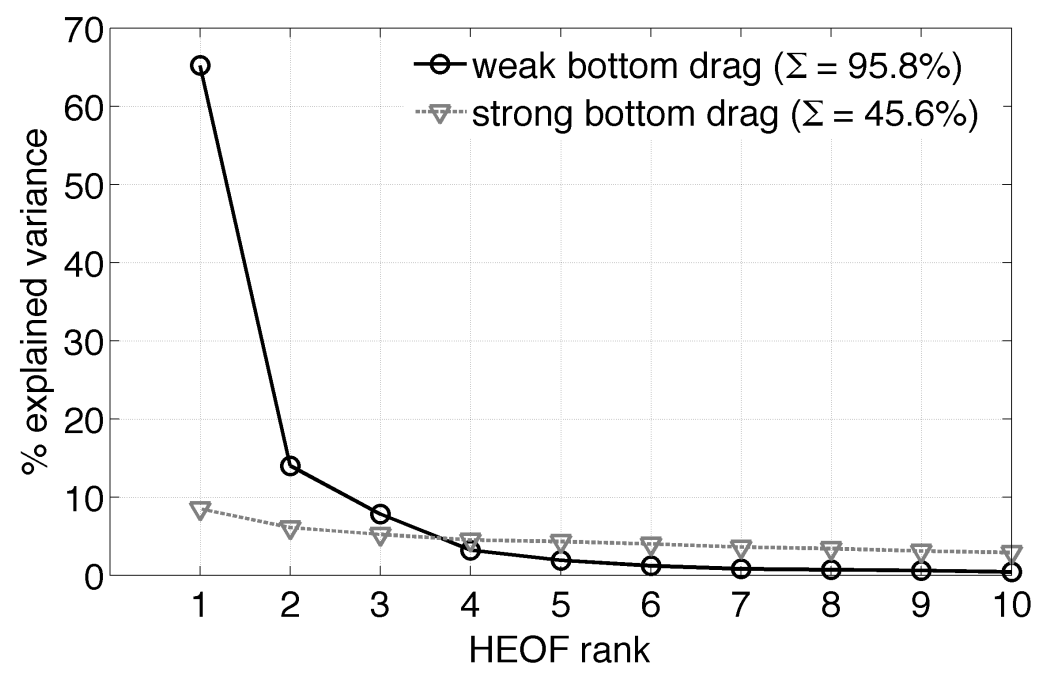

Figure 5-3: Eigenvalues expressed in terms of percentage of explained total variance for the first 10 HEOF modes of the barotropic circulation for the weak $\left(r=10^{-9} s^{-1}\right)$ and strong $\left(r=10^{-7} s^{-1}\right)$ bottom friction cases. The sum $\Sigma$ of the total variance explained by the first 10 HEOFs is given in parentheses. 
of the total variance. It is also very well separated from the next modes, the second one explaining less than $15 \%$ of the variance. Overall, the first 10 modes together account for a little over $95 \%$ of the total variance. All this indicates that 1) the variability of the system projects well on only a couple of statistical modes, and that 2) there is one very dominant, well separated mode that accounts for the majority of the barotropic variability. For the strong bottom friction case on the other hand, the leading mode describes only $9 \%$ of the variance, with the first 10 modes together, less than half of the total variance. Therefore, in this case the HEOF analysis is not successful in identifying a small number of statistical modes on which the system variability projects.

The statistical modes identified by the HEOF analysis represent a set of empirically obtained orthogonal functions on which a given, one hopes large, amount of the system variability projects. However, there are no requirements that these modes correspond directly to any physical process or have any dynamical meaning. It is a necessary next step in the analysis to try to explain the statistical modes and, ideally, relate them to dynamical mechanisms for the variability.

The leading HEOF mode, explaining $65.5 \%$ of the weak bottom friction barotropic variability, is shown in Figure 5-4. For reference, the real and imaginary part of the mode's spatial pattern, representing the oscillation at different phases, are plotted in panels d) and e) of the figure. Alternatively, the same spatial pattern can be expressed in terms of a spatial amplitude $S(x, y)$ and a spatial phase $\theta(x, y)^{*}$, shown in panels a) and b). The spatial amplitude function for the leading mode is spatially inhomogeneous - it possesses two maxima of variability, one in each gyre. The spatial phase function indicates that, at any given time, there is a phase difference of $\pi$ between the oscillation in the northern and southern gyre, or in other words, the oscillation is in opposite phases in the two gyres. Also, the spatial phase increases linearly westward which is indicative of a westward phase propagation at a constant speed. Finally, the power spectrum of the principal component (either its real or

\footnotetext{
*The exact definitions and interpretation of $S(x, y)$ and $\theta(x, y)$ are described in Appendix C.
} 
Spatial amplitude $\mathrm{S}(\mathrm{x}, \mathrm{y})$

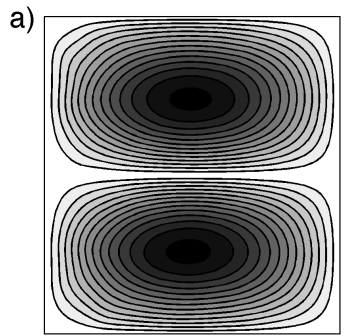

Spatial phase $\theta(x, y)$

b)

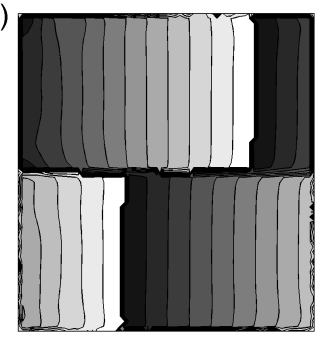

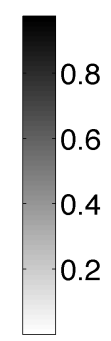

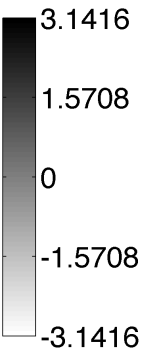

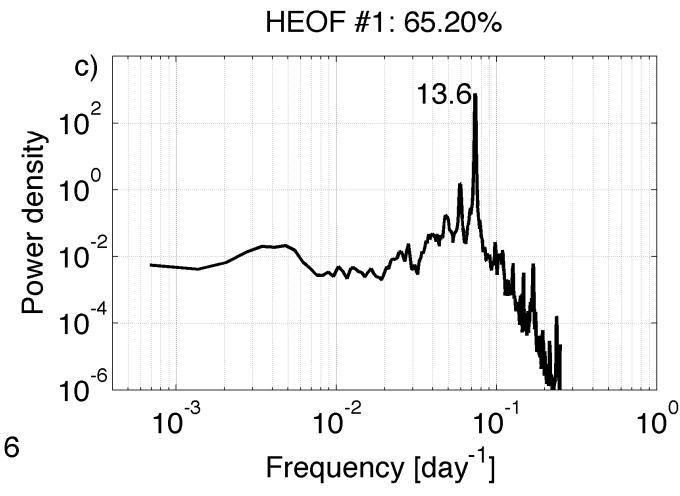

d)

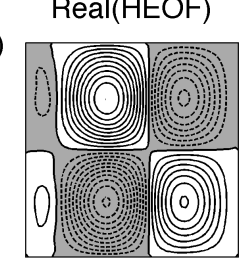

e)

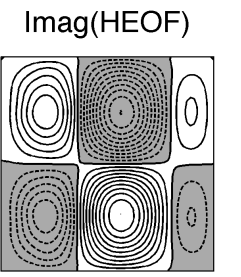

Figure 5-4: First HEOF mode of the barotropic streamfunction in the weak bottom friction case $\left(r=10^{-9} s^{-1}\right)$ explaining $65.2 \%$ of the total variability: a-b, spatial amplitude and phase; c, power spectrum of the real (or imaginary) part of the principal component with period in days indicated for the major peaks; d-e, real and imaginary part of the HEOF showing the oscillation at different phases.

imaginary part) shows only one distinct peak situated at 13.5 days, implying a single frequency oscillation. All these findings - a high-frequency, westward propagating oscillation, that at any given time is in opposite phases in the southern and northern half-basin, are suggestive of a barotropic Rossby basin mode. This will be confirmed in the next section.

For the strong bottom friction case, the leading HEOF explaining 8.5\% of the total variance is plotted in Figure 5-5. The spatial pattern of the mode is significantly different from the one in the weak bottom friction case. Although again the spatial amplitude contains some basin-scale structure, the majority of the variability is concentrated along the midlatitude jet. The pattern is reminiscent of a standing wave with alternating highs and lows along the jet, describing variability associated with meandering of the jet. The spectrum of the principal component reveals several frequencies with periods ranging from 8 days to up to a month, suggesting a variabil- 
Spatial amplitude $S(x, y)$

a)

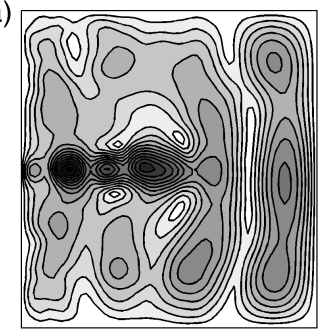

Spatial phase $\theta(x, y)$

b)

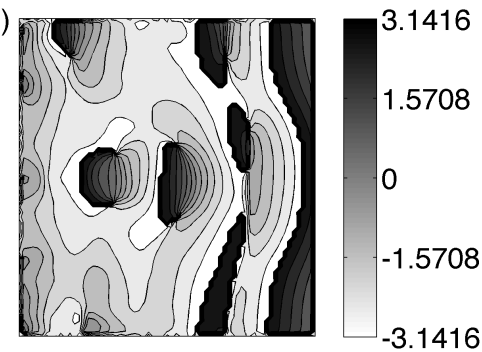

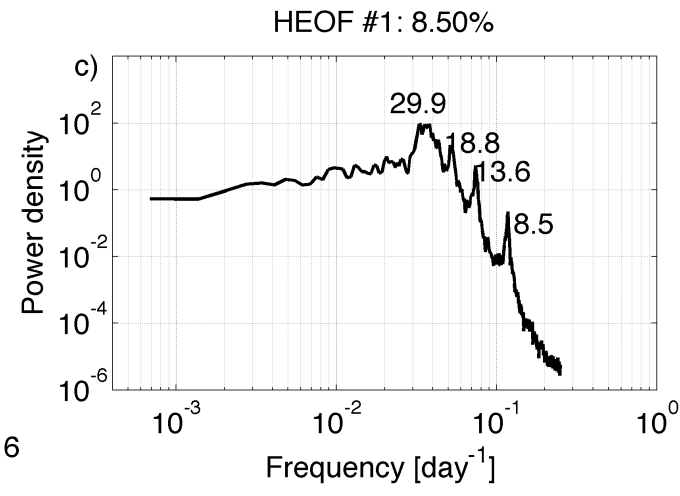

d)

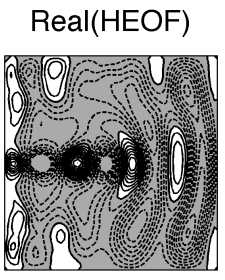

e)
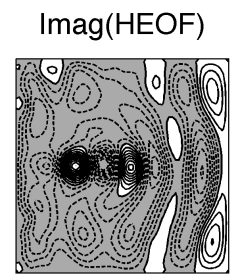

Figure 5-5: First HEOF mode of the barotropic streamfunction in the strong bottom friction case $\left(r=10^{-7} \mathrm{~s}^{-1}\right)$ explaining $8.5 \%$ of the total variance.

ity that is more complex than a single propagating oscillation, as in the weak bottom friction case.

\subsubsection{Barotropic Rossby basin modes}

The barotropic Rossby basin modes are free oscillatory modes of the circulation in a closed basin (Pedlosky, 1987). They are obtained as solutions of the inviscid and unforced linear barotropic potential vorticity equation

$$
\frac{\partial}{\partial t} \nabla^{2} \phi+\frac{\partial \phi}{\partial x}=0
$$

with the boundary condition $\phi=0$ on the walls. The solutions of this equation for a square basin of width one take the form

$$
\phi_{n m}(x, y, t)=\cos \left(\sigma_{n m} t+\frac{x}{2 \sigma_{n m}}\right) \sin (n \pi x) \sin (m \pi y), \quad n, m=1,2, \ldots
$$




\begin{tabular}{|r||r|r|r|r|}
\hline Period [days] & $n=1$ & 2 & 3 & 4 \\
\hline \hline$m=1$ & 8.41 & 13.30 & 18.81 & 24.53 \\
\hline 2 & & 16.83 & 21.45 & 26.61 \\
\hline 3 & & & 25.24 & 29.75 \\
\hline 4 & & & & 33.66 \\
\hline
\end{tabular}

Table 5.2: Period in days for the lowest order barotropic Rossby basin modes in a square basin with size $L=3840 \mathrm{~km}$ and planetary vorticity gradient $\beta_{0}=2 \times$ $10^{-11} m^{-1} s^{-1}$. By definition, $T^{d i m}=4 \pi^{2}\left(n^{2}+m^{2}\right)^{\frac{1}{2}} / L \beta_{0}$.

Here, $n$ and $m$ are respectively the zonal and meridional wavenumbers, and $\sigma_{n m}$, the frequency of the mode satisfying the dispersion relation (non-dimensional form)

$$
\sigma_{n m}=-\frac{1}{2 \pi\left(n^{2}+m^{2}\right)^{\frac{1}{2}}} .
$$

Depending on the wavenumbers, each mode has a certain number of fixed nodes, associated with the sine envelope functions, and a certain number of moving nodes, associated with the cosine carrier wave. The nodes are the locations where the streamfunction is zero and separate cells of opposite direction circulation. What the spatial amplitude function from the HEOF analysis detects, is the number of fixed cells of motion, equal exactly to the wavenumbers $n$ and $m$. For example, the rank 1 HEOF in Figure 5-4 has the structure of a $n=1, m=2$ or $1 \times 2$ basin mode.

The carrier wave is characterized with phase speed $c=-2 \sigma_{n m}^{2}$, i.e. always westward. Thus, the direction of phase propagation found from the spatial phase function for the rank $1 \mathrm{HEOF}$ in Figure 5-4 is also consistent with that of a basin mode.

Finally, from the frequency a period for each mode can be defined, which in dimensional units is

$$
T^{d i m}=\frac{1}{\beta_{0} L} \frac{2 \pi}{\left|\sigma_{n m}\right|}=\frac{4 \pi^{2}}{L \beta_{0}}\left(n^{2}+m^{2}\right)^{\frac{1}{2}} .
$$

The periods of the lowest order barotropic Rossby basin modes in a square basin with width $L=3840 \mathrm{~km}$, are given for reference in Table 5.2. In particular, the theoretical 

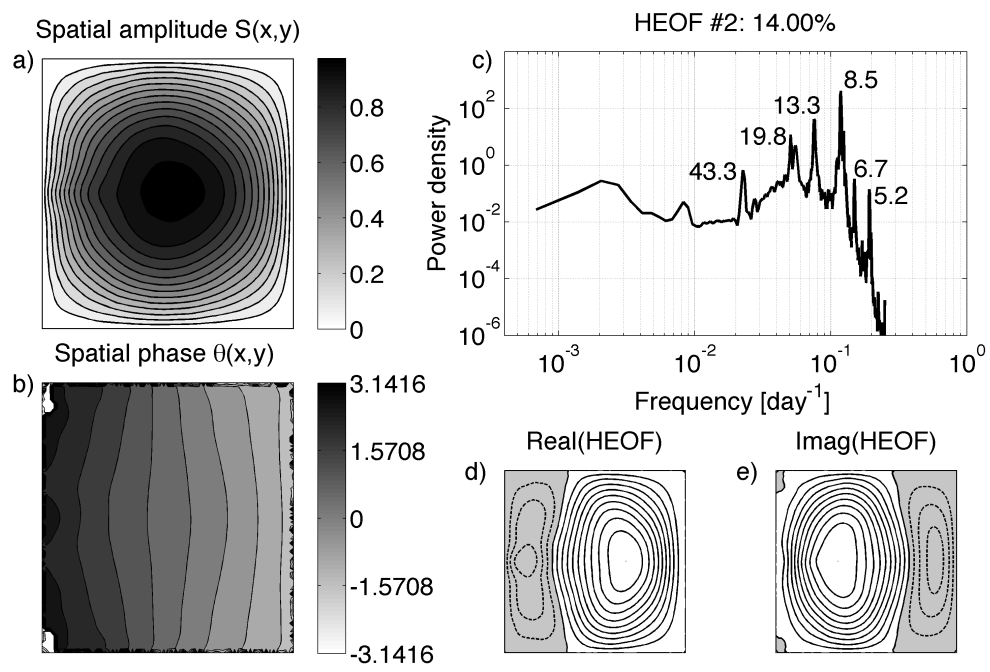

Figure 5-6: Second HEOF of the barotropic streamfunction in the weak bottom friction case $\left(r=10^{-9} s^{-1}\right)$ explaining $14.0 \%$ of the total variance.
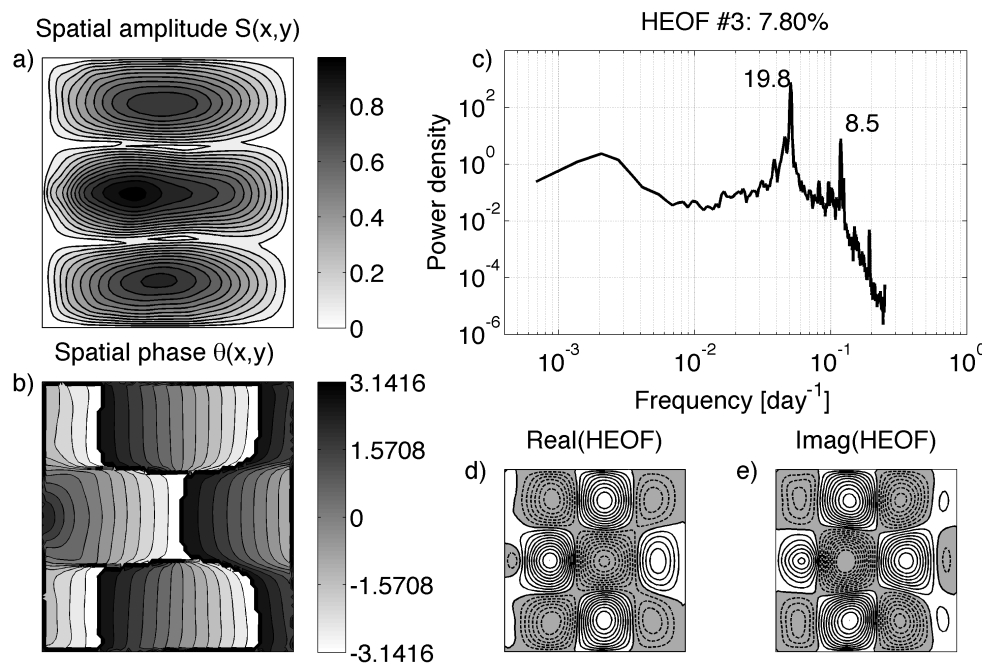

Figure 5-7: Third HEOF mode of the barotropic streamfunction in the weak bottom friction case $\left(r=10^{-9} s^{-1}\right)$ explaining $7.8 \%$ of the total variance.

period for a $1 \times 2$ basin mode is $T=13.3$ days, which closely matches the one found from the power spectrum of the principal component for the rank $1 \mathrm{HEOF}$ in Figure 5-4. Therefore, we can conclude with a large degree of certainty that the majority $(65.2 \%)$ of the barotropic variability in the weak bottom friction case is explained by a resonance of $1 \times 2$ barotropic Rossby basin mode. 
The next two HEOF modes for the weak bottom friction case are shown in Figures 5-6 and 5-7, respectively. They both have the spatial pattern of Rossby basin modes - more specifically, a $1 \times 1$ and a $1 \times 3$ basin mode. Both oscillations are characterized with westward phase propagation, again consistent with the direction of phase propagation for a basin mode. However, unlike the leading HEOF, none of these statistical modes manages to isolate a single basin mode. As evident from the multiple spectral peaks of the corresponding principal components spectra, both HEOFs represent rather a mix of different basin modes, with one in particular being dominant.

Concerning the strong bottom friction case, although the HEOF analysis did not extract any variability in the form of basin modes, high-frequency spectral peaks consistent with the period of Rossby basin modes were identified in the barotropic streamfunction power density in Figure 5-2. However, if the barotropic streamfunction is filtered around each of these frequencies prior to performing the HEOF analysis, then the corresponding Rossby basin mode is identified as the leading pattern explaining the variability of the filtered data series. Therefore, even when stronger bottom drag is applied the basin modes are present, but their amplitude is strongly attenuated and they do not dominate the system variability.

The question of whether barotropic basin mode variability is relevant for the real ocean circulation does not have a definitive answer. One reason for that is that the basin modes are very challenging to observe. Because of their fast timescales, small amplitudes and large spatial scales they are difficult to detect in global altimetry data sets so that even their existence is under question. There have been nevertheless some recent direct observations of oscillations identified as barotropic basin modes. Usually, these observations come from closed or semi-enclosed by bathymetry basins characterized with strong overall level of variability.

Probably the region in the World Ocean where barotropic basin modes have been identified with the greatest degree of certainty is the Argentine basin in the South Atlantic ocean. This is a region characterized with very high degree of sea surface 
height variability. Part of it is due to the intense eddy and frontal activity resulting from confluent currents, part of it has been identified in several measurements coming from altimetry (Fu et al., 2001), current meters (Weatherly, 1993) and pressure gauges (Hughes et al., 2007), as a barotropic oscillation with a 20-30 days period. Most recently, different statistical techniques applied to a decade of weekly SSH maps suggests that actually multiple basin modes are present in the Argentine basin (Weijer et al., 2007a). Matching the real ocean basin oscillations with their theoretical counterpart is challenging since they are highly distorted by the bottom bathymetry. However, using numerical simulations it can be shown that at least some of the modes of variability identified be the statistical analysis are consistent with low-order barotropic basin modes (Weijer et al., 2007b).

Oscillations identified as barotropic Rossby basin modes have also been found in the Mascarene basin in the Indian Ocean. A signal with westward propagation and a period around 60 days has been isolated from an array of current meters (Warren et al., 2002). In a related work (Weijer, 2008), the normal modes of a motionless barotropic shallow water model with realistic bathymetry are computed. Several modes with monthly to bimonthly timescales are identified, which supports the current meter measurement interpretation as resonantly excited Rossby basin modes.

Barotropic Rossby basin modes are considered to be an important part as well of the circulation in semi-enclosed seas. For example, the Black Sea, being a nearly closed basin, features favorable conditions for the excitations of resonant oscillations. Although specific direct observations of basin modes are not available, indications for basin oscillations are present in the Black Sea literature. Several studies using numerical model address this topic. Stanev and Rachev (1999) and Stanev and Staneva (2000) investigate the existence of basin modes and examine their impact on the Black Sea circulation using an eddy-resolving model with realistic bathymetry and forcing. It is found that basin modes are an important part of the system variability, especially in the Black Sea interior, and are responsible for enhanced baroclinic 
variability, modified eddy field and tracer dispersion, and could lead to transitions between different quasi-steady states of the circulation.

\subsubsection{Main questions}

The analysis carried so far on the strongly nonlinear thermally-forced circulation allowed us to conclude that variability in the form of barotropic Rossby basin modes is produced. Several characteristics of the circulation have been identified that seem to be directly related to the extent to which the system variability is dominated by the basin modes:

1. For weak bottom friction or strong basin modes, the time-mean barotropic circulation consists of a basin-scale 4-gyre flow, plus in addition small and relatively weak recirculations near the western wall.

2. For strong bottom friction or weak basin modes, the time-mean barotropic circulation consists of strong and large in zonal extent inertial recirculations. There is no basin-scale time-mean barotropic circulation.

3. The time-mean baroclinic circulation consists of a double-gyre flow with no inertial recirculations. Its variability in the weak bottom friction limit shows spectral peaks at the frequencies of the Rossby basin modes that dominate the barotropic variability.

In the remainder of this chapter, more detailed analysis will be carried out in order to explain the findings listed above. In particular, emphasis will be put on the following aspects:

(a) Mean flow driven by the basin modes (Section 5.3).

(b) Baroclinic variability driven by the basin modes (Section 5.4).

(c) Basin mode effect on the inertial recirculations (Section 5.5).

(d) Heat budget of the system in the time-dependent regime (Section 5.6). 


\subsection{Rectification of mean flow by the basin modes}

The hypothesis that we want to verify in this section is that the multiple gyre timemean barotropic circulation diagnosed in the weak bottom friction case is driven by the basin modes.

\subsubsection{Mean flow driven by the basin modes}

Let us assume a circulation that is barotropic and governed by inviscid dynamics. Let the circulation be described at leading order by a $n \times m$ basin mode plus some small amplitude perturbation flow,

$$
\phi(x, y, t)=\phi^{(0)}(x, y, t)+\epsilon \phi^{(1)}(x, y, t), \quad \epsilon \ll 1,
$$

where

$$
\phi^{(0)}(x, y, t)=A_{n m} \cos \left(\sigma_{n m} t+\frac{x}{2 \sigma_{n m}}\right) \sin (n \pi x) \sin (m \pi y),
$$

with $\sigma_{n m}=-1 /\left(2 \pi \sqrt{n^{2}+m^{2}}\right)$ and $A_{n m}$ designating the amplitude of the basin mode.

The circulation at leading order satisfies the unforced and inviscid linear vorticity equation

$$
\frac{\partial}{\partial t} \nabla^{2} \phi^{(0)}+\frac{\partial \phi^{(0)}}{\partial x}=0
$$

In writing this equation we have assumed that $\delta_{I}^{2} \approx \epsilon$, so that the nonlinear advection of relative vorticity $\delta_{I}^{2} J\left(\phi^{(0)}, \nabla^{2} \phi^{(0)}\right)$ is omitted and does not affect the dynamics at this order. Instead, it serves as forcing for the next order circulation $\phi^{(1)}(x, y, t)$

$$
\frac{\partial}{\partial t} \nabla^{2} \phi^{(1)}+\frac{\partial \phi^{(1)}}{\partial x}=-\frac{\delta_{I}^{2}}{\epsilon} J\left(\phi^{(0)}, \nabla^{2} \phi^{(0)}\right)
$$

Furthermore, if this equation is averaged over the period of the basin mode, the following relationship for the rectified flow is obtained

$$
\frac{\partial}{\partial x} \overline{\phi^{(1)}}=-\frac{\delta_{I}^{2}}{\epsilon} \overline{J\left(\phi^{(0)}, \nabla^{2} \phi^{(0)}\right)} .
$$




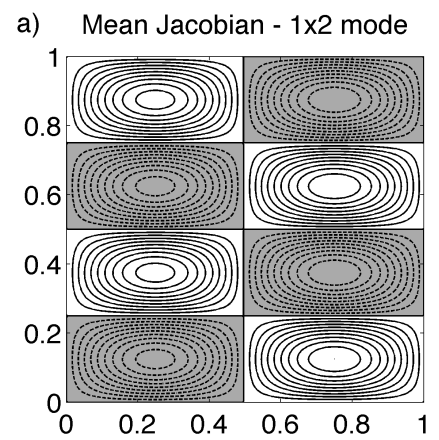

d) Rectified mean flow - 1×2 mode

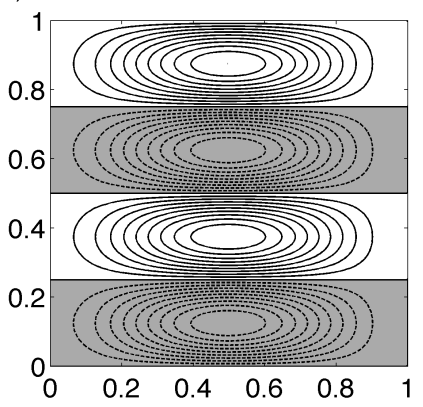

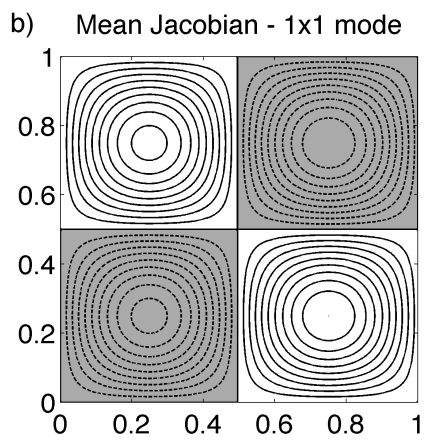

e) Rectified mean flow - 1x1 mode

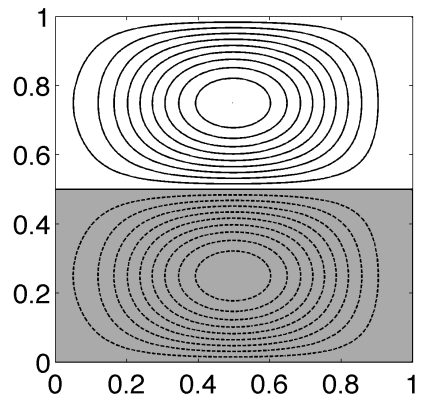

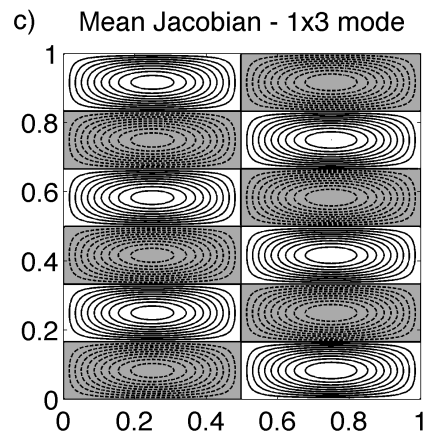

f) Rectified mean flow - 1×3 mode

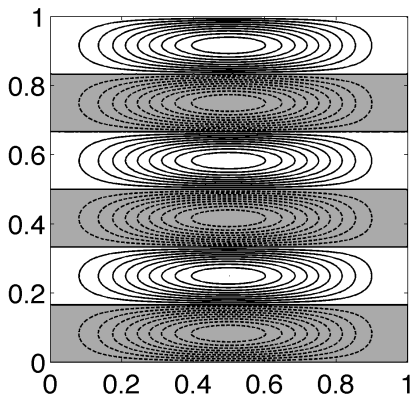

Figure 5-8: Illustration of mean flow driven by a $1 \times 2,1 \times 1$ and $1 \times 3$ Rossby basin mode: a-c, the time-mean Jacobian $-\overline{J\left(\phi^{(0)}, \nabla^{2} \phi^{(0)}\right)}$ driving the circulation; d-e, the rectified time-mean circulation $\overline{\phi^{(1)}}$. Each plotted field is scaled by its maximum value. The contour interval is 0.1 .

Thus, although the basin mode flow itself averages to zero over one period, because of the nonlinear dynamics its momentum flux is non-zero, and is consequently able to drive a time-mean flow. This topic has been previously discussed in the context of wind-driven circulation resulting from time-dependent wind stress (Pedlosky, 1965).

In Figure 5-8(a-d) the time-averaged Jacobian and corresponding rectified timemean circulation as described by Eq.(5.8) are shown for the case of a $1 \times 2$ Rossby basin mode. This mode has been identified as explaining $65 \%$ of the barotropic circulation variability in the weak bottom friction case. The $1 \times 2$ mode drives a mean circulation consisting of 4 gyres with alternating flow direction. This circulation bears similarity to the time-mean barotropic circulation diagnosed in the case of weak bottom friction (Figure 5-1(A)). One difference is that the basin mode rectified flow does not feature the western boundary current flow, nor the inertial recirculations. Also, the $1 \times 2$ basin mode drives gyres that are identical in size and strength, while for the time- 
mean barotropic circulation in Figure 5-1(A) the two outer gyres are stronger and smaller in meridional extent compared to the two inner gyres.

One reason for that asymmetry could be that other basin modes contribute to the barotropic variability of the system. In Figure $5-8$ the flow rectified by the $1 \times 1$ and $1 \times 3$ basin modes is shown as well. These two modes have been identified as explaining respectively an additional $14 \%$ and $8 \%$ of the barotropic circulation variability in the weak bottom friction case. It can be seen that the $1 \times 1$ mode drives a 2-gyre flow, while $1 \times 3$ mode, a 6 -gyre flow. If added to the 4-gyre flow driven the most dominant $1 \times 2$ mode, the effect of the $1 \times 1$ and the $1 \times 3$ mode rectified flow will be to distort the otherwise symmetrical 4-gyre pattern. It can be inferred from Figure 5-8, that the momentum flux from the $1 \times 1$ basin mode will tend to intensify and expand meridionally the outer gyres, while the momentum flux from the $1 \times 3$ basin mode will tend to intensify and contract meridionally the outer gyres. Thus, the resulting net time-mean circulation when several basin modes act together will be less symmetrical.

\subsubsection{Mean flow driven by an oscillating patch of wind stress}

In the analysis in the previous section, it was assumed that there is a leading order flow in the form of a Rossby basin mode without specifying how it was excited. Also, all frictional forces were neglected. Another approach to the same problem would be to apply a forcing that selectively excites and sustains against dissipation a specific basin mode. For completeness, this approach is considered in this section.

The forcing of choice is an oscillating wind stress. The wind stress is limited to a square patch with area equal to a quarter of the total basin area, centered in the middle of the basin (Figure 5-9). Forcing on a global scale is not necessary in order to excite basin resonances. However, in order to excite selectively a specific Rossby basin mode, the period of the oscillating Ekman velocity should be close to the one of the basin mode. The following form for the Ekman pumping velocity within the 


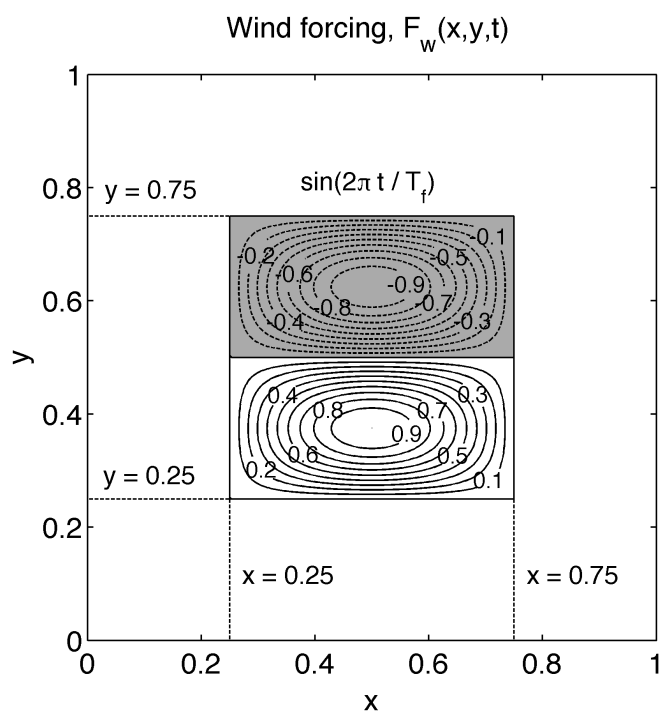

Figure 5-9: Spatial structure of the Ekman pumping velocity for the oscillating wind forcing. The spatial distribution of the forcing remains unchanged, while its amplitude oscillates sinusoidally in time with period $T_{f}$.

wind patch was chosen,

$$
F_{w}(x, y, t)=\sin \left(\frac{2 \pi t}{T_{f}}\right) \sin \left[2 \pi\left(x-\frac{1}{4}\right)\right] \sin \left[4 \pi\left(y-\frac{1}{4}\right)\right], \quad x, y \in\left[\frac{1}{4}, \frac{3}{4}\right]
$$

Outside the patch, the Ekman pumping amplitude is set to zero, while within the patch $U_{w}=1$, i.e. the velocity scale $U$ is set by the wind stress. We are using $U=$ $\tau_{0} / \rho_{0} \beta_{0} H_{1}=0.05 \mathrm{~ms}^{-1}$, which corresponds to a wind stress magnitude of $0.15 \mathrm{Nm}^{-2}$. The period of the applied forcing is either $T_{f}=13.00$ days or $T_{f}=13.25$ days, both close to the theoretical period of the $1 \times 2$ basin mode, $T_{1 \times 2}=13.30$ days .

With this choice for the Ekman pumping velocity, there is no net vorticity input into the basin from the wind forcing. At any given time, the Ekman pumping velocity over the wind patch averages to zero. Also, at any given location, the Ekman pumping velocity over one forcing period averages to zero as well. However, because of the excitation of basin resonances a time-mean circulation is generated.

The power density spectra of the instantaneous barotropic and baroclinic streamfunctions sampled every day over a 27-year period after the initial spin-up are shown 

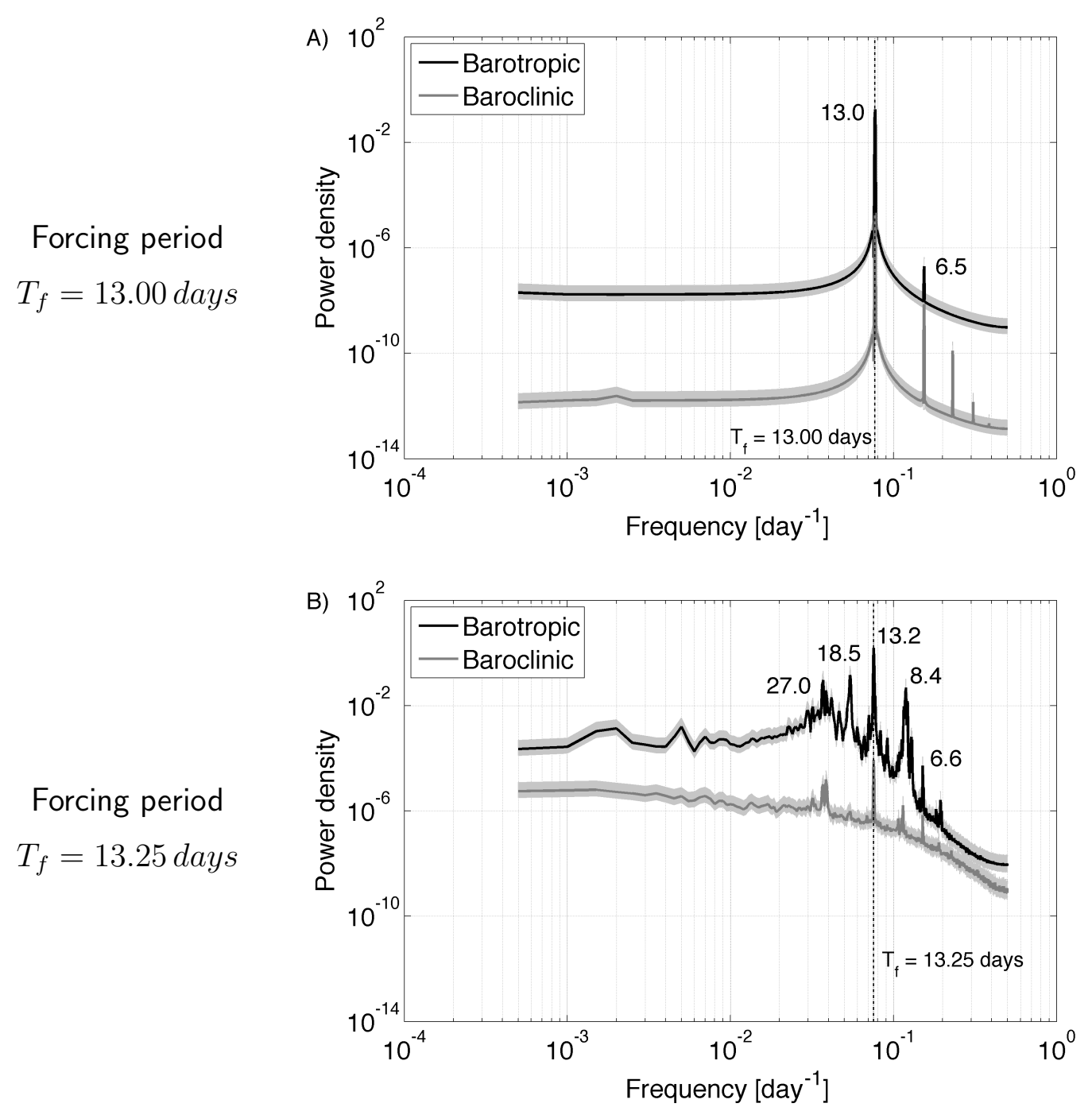

Figure 5-10: Power density spectrum with a 95\% confidence interval of the instantaneous barotropic and baroclinic streamfunctions for the oscillating wind forcing with period: A), $T_{f}=13.00$ days; B), $T_{f}=13.25$ days. The period, in days, for the largest spectral peaks is indicated. The dotted line indicates the frequency of the forcing.

in Figure 5-10. For the oscillating forcing with period $T_{f}=13.00$ days, the major spectral peak for both the barotropic and baroclinic streamfunctions is at the forcing frequency, with some harmonics present as well. From the leading HEOF for the barotropic streamfunction plotted in Figure 5-11 it can be seen that $100 \%$ of the barotropic variability is explained by a $1 \times 2$ Rossby basin mode. Thus indeed, the oscillating wind forcing excites selectively the $1 \times 2$ basin mode. Since the entire 

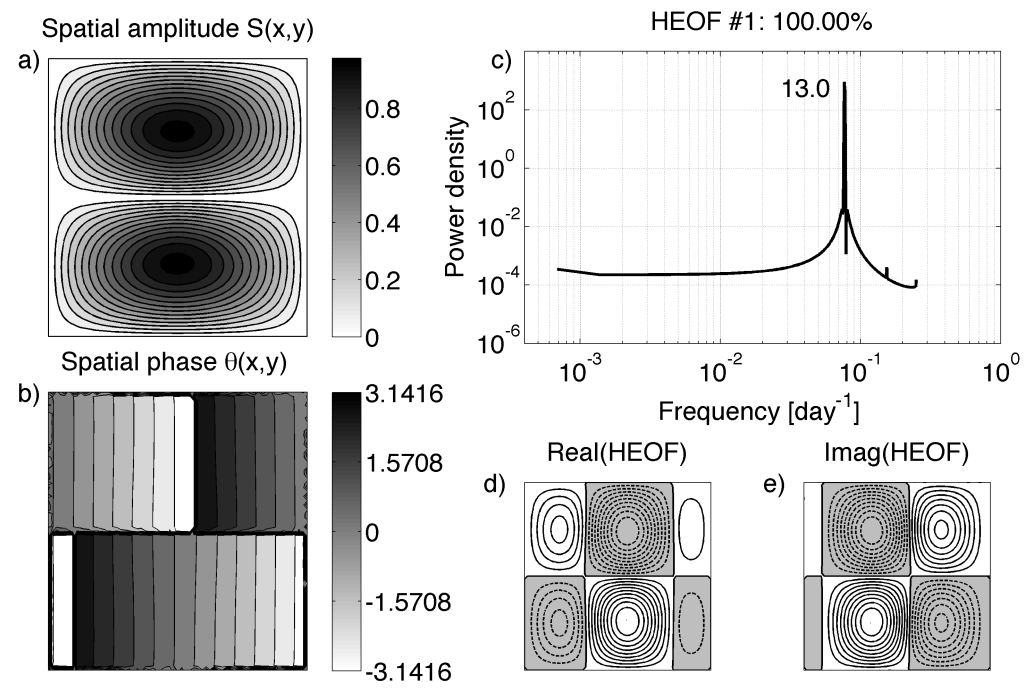

Figure 5-11: Leading HEOF mode of the barotropic streamfunction for the oscillating wind forcing with period $T_{f}=13.00$ days.
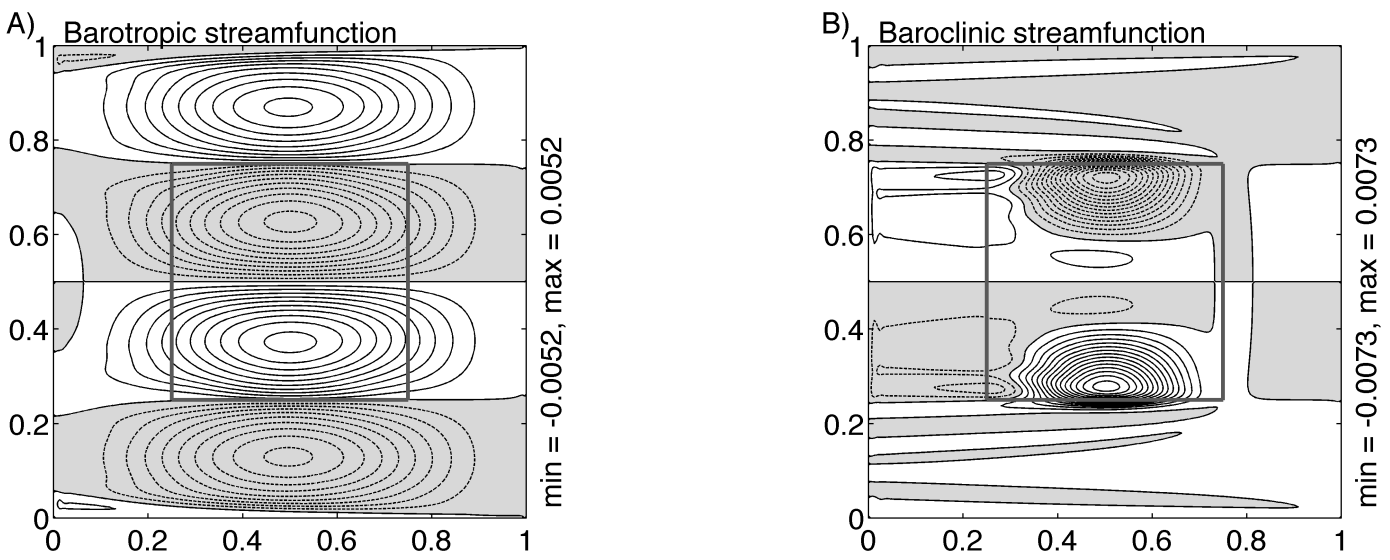

Figure 5-12: Time-mean circulation for the oscillating wind forcing with period $T_{f}=13.00$ days: A) barotropic streamfunction (contour interval 0.005); B) baroclinic streamfunction (contour interval 0.0025). The streamfunction is scaled with the maximum linear baroclinic streamfunction $\tau^{(\text {lin })}=\delta_{T} U_{E}\left(1-e^{-\frac{1}{\delta_{T}}}\right) U L=0.12 \times 10^{6} m^{2} s^{-1}$.

variability is accounted for by a single basin mode, it can be concluded that the circulation, although time-dependent because of the oscillating forcing, is otherwise laminar - there are no instabilities or nonlinear interactions between different modes.

The resulting time-mean circulation by vertical modes is shown in Figure 5-12. The circulation is predominantly barotropic and consists of the familiar 4-gyre flow. 

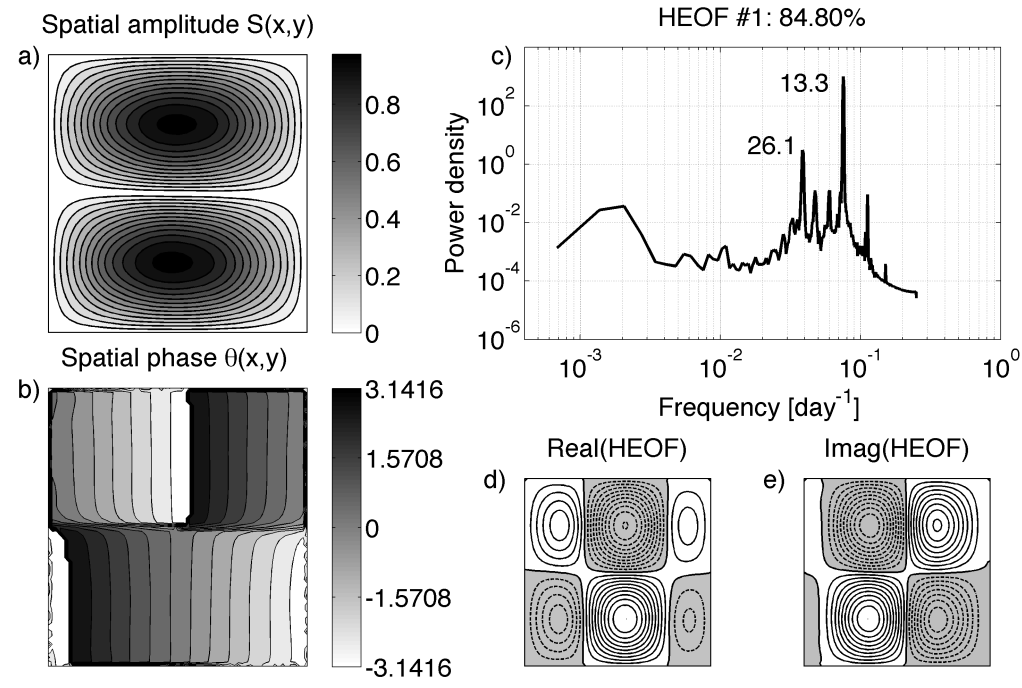

Figure 5-13: Leading HEOF mode of the barotropic streamfunction for the oscillating wind forcing with period $T_{f}=13.25$ days.
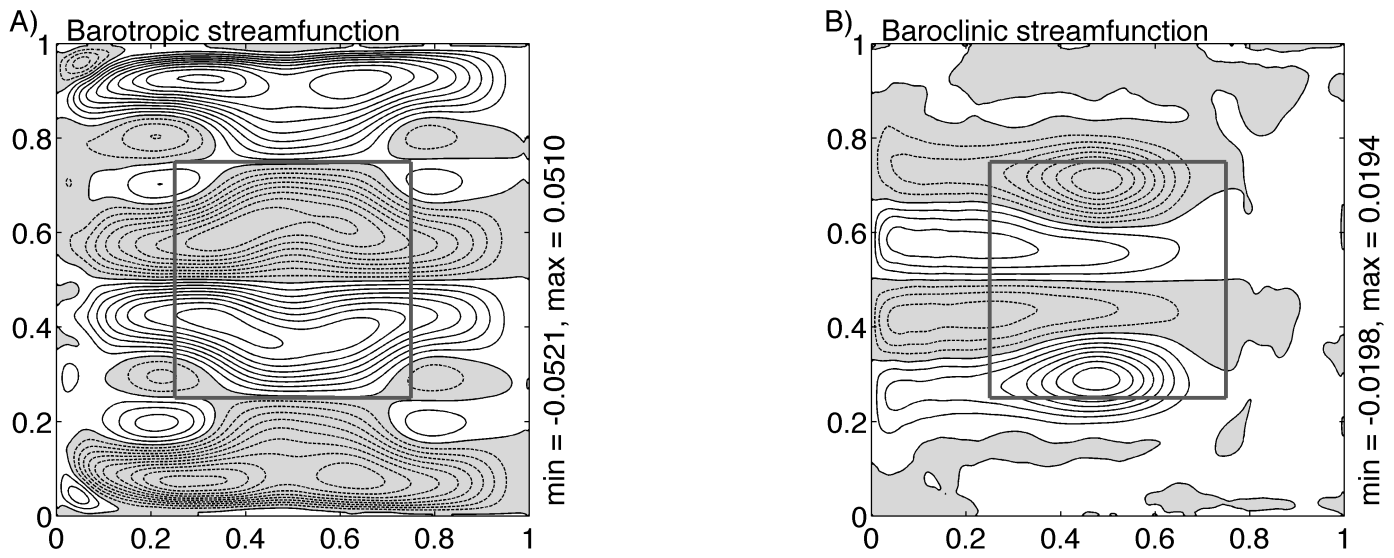

Figure 5-14: Time-mean circulation for the oscillating wind forcing with period $T_{f}=13.25$ days: A) barotropic streamfunction (contour interval 0.005); B) baroclinic streamfunction (contour interval 0.0025). The streamfunction is scaled with the maximum linear baroclinic streamfunction $\tau^{(\text {lin })}=\delta_{T} U_{w}\left(1-e^{-\frac{1}{\delta_{T}}}\right) U L=0.12 \times 10^{6} m^{2} s^{-1}$.

Since the circulation is driven by the resonating $1 \times 2$ basin mode, it extends outside of the directly wind-forced region. There is no western boundary layer and no inertial recirculations. The gyres driven by the oscillating wind patch appear identical in strength and size. As already mentioned, this can be due to the fact that a single basin mode, and not a combination of several basin modes is excited as in the strongly 
nonlinear thermally-forced ocean.

For comparison, the circulation driven by an oscillating wind forcing with the same amplitude, but with a slightly longer period $T_{f}=13.25$ days, is examined as well. Since the period of the forcing is closer to the theoretical basin mode period $T_{1 \times 2}=$ 13.30 days, the system is closer to resonance leading to larger velocities fluctuations. The resulting flow is not laminar anymore. This is evident from the overall greater level of variance of the flow, as seen in the power density spectrum in Figure 510(B). Also, although the major spectral peak for both the barotropic and baroclinic flow is again at the forcing frequency, there are several other, non-harmonic peaks. Their periods are suggestive of basin modes, other than the $1 \times 2$, being excited as well. Indeed, the HEOF analysis reveals that only $84 \%$ of the barotropic variability is explained by the $1 \times 2$ basin mode (Figure $5-13$ ). The second HEOF mode (not shown) accounts for $7 \%$ of the variance and has the spatial structure and propagating properties of a $2 \times 4$ Rossby basin mode. Nevertheless, the resulting time-mean barotropic circulation still bears the familiar form of a 4-gyre flow, although it is strongly distorted (Figure 5-14). Possible causes for that could be the non-linear interactions between the different basin modes or also eddy-driven flow.

\subsection{Baroclinic variability driven by the basin modes}

It was determined that in the weak bottom friction case, when the system variability is dominated by barotropic Rossby basin modes, there are distinct peaks in the spectrum of the baroclinic streamfunction at frequencies close to those of the basin modes. In this section, we want to verify the hypothesis that these peaks correspond to variability of the baroclinic flow directly driven by the basin modes.

The most important statistical modes of baroclinic variability in the weak bottom friction case are diagnosed from a 20-year calculation. The instantaneous baroclinic streamfunction is subsampled, as for the barotropic HEOF analysis, every 2 days on a coarse $65 \times 65$ grid, giving a resolution of $\Delta x=60 \mathrm{~km}$. The two most important 


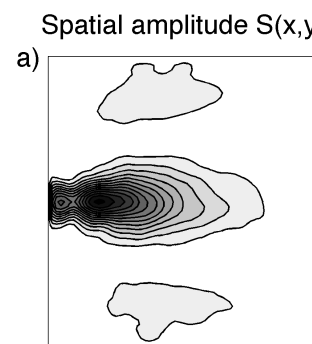

Spatial phase $\theta(x, y)$

b)

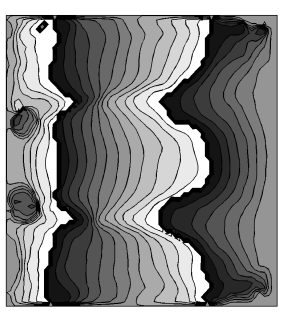

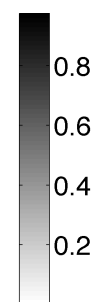

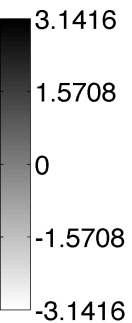

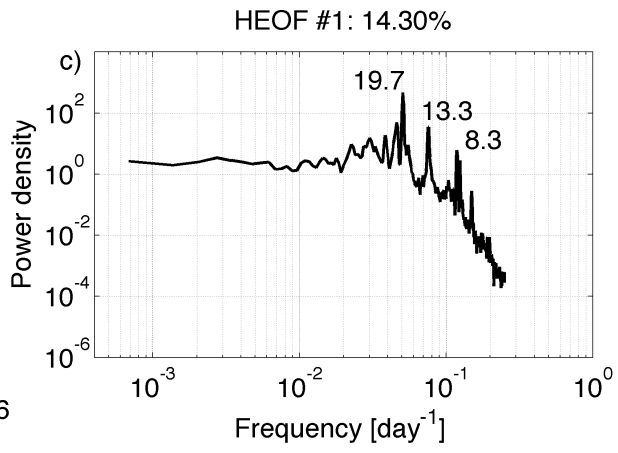

d)

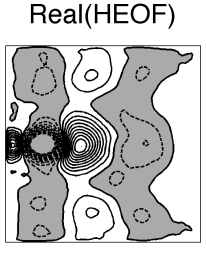

e)
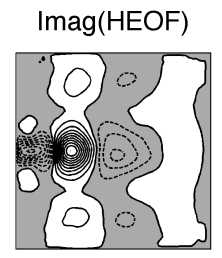

Figure 5-15: First HEOF mode of the baroclinic streamfunction in the weak bottom friction case $\left(r=10^{-9} \mathrm{~s}^{-1}\right)$ explaining $14.3 \%$ of the total variance.

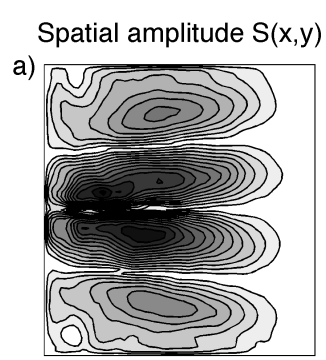

Spatial phase $\theta(x, y)$

b)

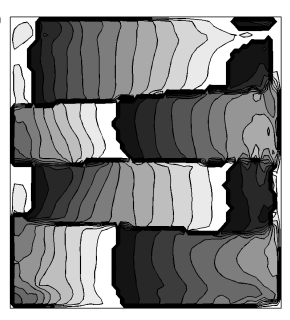

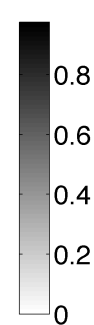
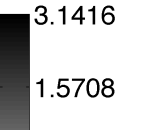

0

$-1.5708$

$-3.1416$
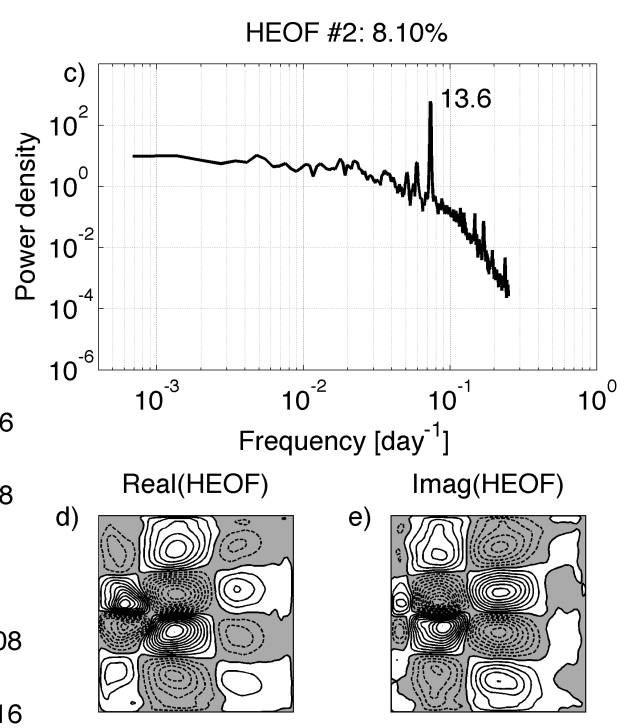

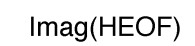

e)

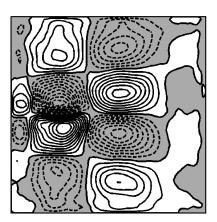

Figure 5-16: Second HEOF mode of the baroclinic streamfunction in the weak bottom friction case $\left(r=10^{-9} s^{-1}\right)$ explaining $8.1 \%$ of the total variance.

statistical modes explaining a little over $22 \%$ of the system variability are shown in Figures 5-15 and 5-16.

The majority of the variability, especially for the leading mode, is concentrated along the midlatitude jet with the real and imaginary part of the HEOF showing 
alternating lows and highs, indicative of meandering of the jet. The timescales associated with the variability are rather short - the spectra of the principal components (either the real or imaginary part) contain several peaks, all corresponding to periods less than 20 days, consistent with the timescales for low-order basin modes. Further confirming a possible link to the basin modes is the fact that the baroclinic variability displays basin-scale structure, especially the part explained by the 2nd HEOF. Also, the spatial phase for both HEOFs shows a general westward direction of phase propagation, similar to the one for the Rossby basin modes.

In order to separate the possible contributions from the basin modes, the instantaneous baroclinic streamfunction is filtered around the frequencies of the two major peaks evident in the power density spectrum of the baroclinic streamfunction (Figure 5-2(A)). The periods corresponding to these two peaks, 13 and 18 days, closely match the periods of the two most dominant basin modes, the $1 \times 2$ and the $1 \times 3$ mode, respectively. A HEOF analysis of the filtered baroclinic streamfunction is then performed with the results shown in Figures 5-17(A) and 5-19(A), respectively. In each case, a single mode explains over $60 \%$ of the variance of the filtered data, with the next one (not shown), less than 15\%. The fact that the variability of the filtered baroclinic flow is accounted for in its majority by a single statistical mode is encouraging for finding a simple dynamical explanation for its origin. It is worth pointing out that the HEOF explaining the variability of the baroclinic flow filtered around the period of the most important $1 \times 2$ basin mode is very similar to the 2 nd HEOF mode of the full, unfiltered data. The HEOF explaining the variability of the baroclinic flow filtered around the period of the second most important $1 \times 3$ basin mode, is basically the same as the 1st HEOF mode of the unfiltered data. This is consistent with the idea that the high-frequency part of the baroclinic variability is directly forced by the dominant basin modes.

In order to confirm that idea, it is helpful to examine an expansion of the governing equations. Suppose that at leading order the circulation is composed of a time-mean baroclinic flow $\bar{\tau}(x, y)$ and a barotropic flow in the form of a basin mode $\phi_{n m}(x, y, t)$, 
so that

$$
\begin{aligned}
\phi(x, y, t) & =\phi_{n m}(x, y, t), \\
\tau(x, y, t) & =\bar{\tau}(x, y)+\epsilon \tau^{\prime}(x, y, t), \quad \epsilon \ll 1 .
\end{aligned}
$$

What we want to establish is the spatial and temporal structure of the baroclinic perturbation flow $\tau^{\prime}(x, y, t)$ driven by the presence of a basin mode.

When considering the interior large-scale ocean circulation, the relative vorticity contribution to the potential vorticity can be neglected. This means that the barotropic potential vorticity is simply equal to the background planetary vorticity, while the baroclinic potential vorticity is represented by the vortex stretching, and is thus proportional to the interface displacement $\eta=-\tau$. This leads to the following simplification of equation (3.26), governing the baroclinic potential vorticity evolution

$$
\frac{\partial}{\partial t}(-F \tau)+\delta_{I}^{2} J(\phi,-F \tau)+\frac{\partial \tau}{\partial x}=\frac{\tau+U_{T} F_{T}(y)}{\delta_{T}}+\delta_{M}^{3} \nabla^{4} \tau+\delta_{S} \nabla^{2}(\phi-\delta \tau)
$$

If we assume that the nonlinear terms are small so that $\delta_{I}^{2} \approx \epsilon$, the leading order baroclinic circulation $\bar{\tau}(x, y)$ satisfies a linear vorticity balance between advection of planetary vorticity, thermal forcing input and dissipation of relative vorticity

$$
\frac{\partial \bar{\tau}}{\partial x}=\underbrace{\frac{1}{\delta_{T}}\left(\bar{\tau}+U_{T} F_{T}(y)\right)}_{\text {Thermal forcing }}+\underbrace{\delta_{M}^{3} \nabla^{4} \bar{\tau}+\delta_{S} \nabla^{2}(-\delta \bar{\tau})}_{\text {Dissipation }} .
$$

The inertial advective terms involving the leading order circulation missing in this equation, appear instead as forcing for the perturbation flow $\tau^{\prime}$, so that

$$
\frac{\partial}{\partial t}\left(-F \tau^{\prime}\right)+\frac{\partial \tau^{\prime}}{\partial x}=\underbrace{-\frac{\delta_{I}^{2}}{\epsilon} J\left(\phi_{n m},-F \bar{\tau}\right)}_{\text {Basin mode forcing }}+\underbrace{\frac{\tau^{\prime}}{\delta_{T}}+\delta_{M}^{3} \nabla^{4} \tau^{\prime}+\delta_{S} \nabla^{2}\left(\frac{1}{\epsilon} \phi_{n m}-\delta \tau^{\prime}\right)}_{\text {Interfacial friction, Dissipation }} .
$$

The perturbation flow is driven thus by the momentum flux coming from the advection of the mean baroclinic vorticity by the basin mode. Note that the thermal forcing 
appears in the perturbation equation as restoring toward the no motion state $(h=0)$, which has the effect of an additional dissipation in the form of interfacial friction.

From all the terms involved in the perturbation equation above there are two that dominate the dynamics, which leads to the following simplified balance

$$
\frac{\partial}{\partial t}\left(-F \tau^{\prime}\right)=-\frac{\delta_{I}^{2}}{\epsilon} J\left(\phi_{n m},-F \bar{\tau}\right)
$$

Therefore, it is the basin modes that drive the perturbation baroclinic flow by advecting the background baroclinic potential vorticity, set by the mean interface displacement. They force thus directly baroclinic variability on the same short timescales as their periods

This statement can be tested using the time-mean interface displacement obtained in the weak bottom friction case. Although in this case the time-mean circulation $\bar{\tau}(x, y)$ does not satisfy the linear balance given by Eq.(5.14), it can be thought as satisfying a modified balance, where an additional residual forcing $\mathcal{F}$ is included accounting for all the neglected effects of eddy and mean fluxes. With this additional adjustment in mind, all the derivations for the basin mode driven perturbation baroclinic flow still hold.

The spatial amplitude and phase from the HEOF analysis of the perturbation baroclinic circulation $\tau^{\prime}(x, y, t)$, resulting from advecting the mean interface displacement shown in Figure 5 -1(C) by either the $1 \times 2$ or $1 \times 3$ basin mode, are shown respectively in Figures 5-17(B) and 5-19(B). Both are basically the same as the spatial amplitude and phase from the HEOF analysis of the filtered baroclinic streamfunction around the respective mode frequency (Figures 5-17(A) and 5-19(A)). Therefore, the high-frequency baroclinic variability in the weak bottom friction case is indeed directly forced by the basin modes dominating the system variability.

Concerning the spatial structure of the basin-mode driven variability, it is characterized with large spatial scales. Figures 5-18 and 5-20 illustrate the tendency term $-J\left(\phi_{n m}, \bar{\tau}\right)$ for the baroclinic variability forced respectively by the $1 \times 2$ and 
I) Filtered baroclinic HEOF

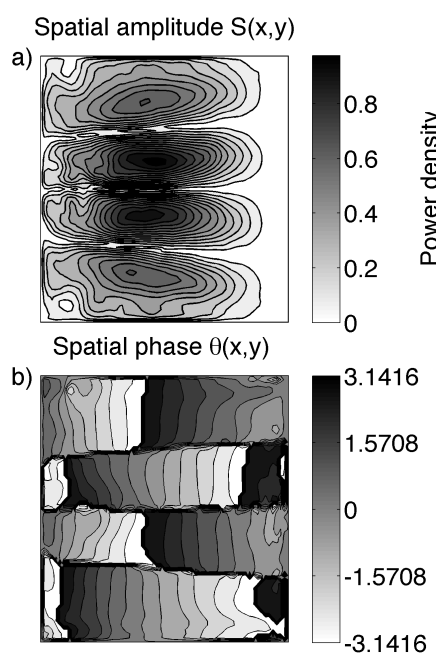

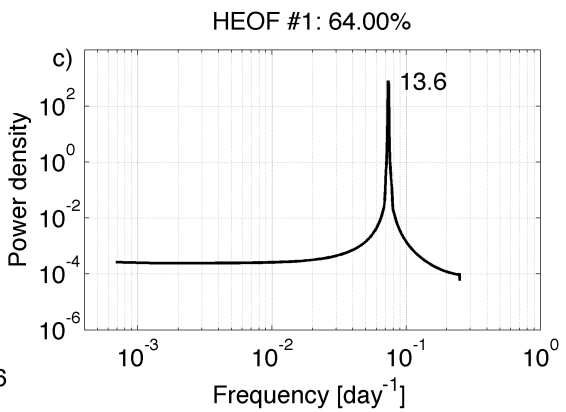

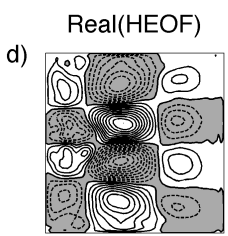

e)

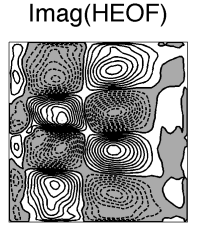

II) Perturbation flow

Spatial amplitude $\mathrm{S}(\mathrm{x}, \mathrm{y})$
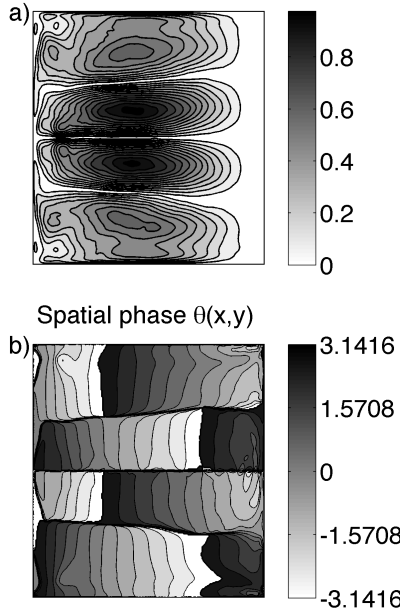

Figure 5-17: I) First HEOF mode of the baroclinic streamfunction in the weak bottom friction case $\left(r=10^{-9} \mathrm{~s}^{-2}\right)$ filtered around the period of the most dominant $1 \times 2$ barotropic basin mode, $T_{1 \times 2}=13.30$ days. II) Spatial amplitude and phase function for the baroclinic perturbation circulation driven by the basin mode.

Mean baroclinic $\Psi_{\mathrm{BC}}$
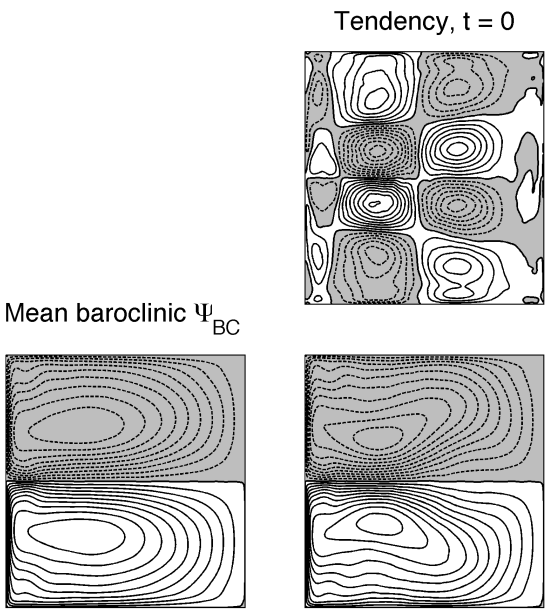
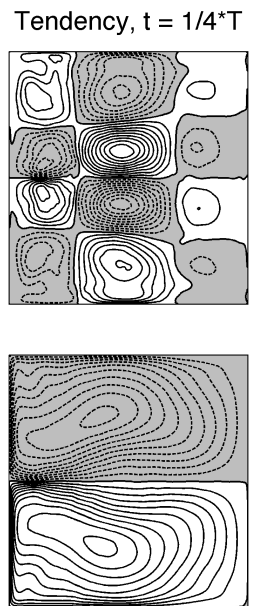
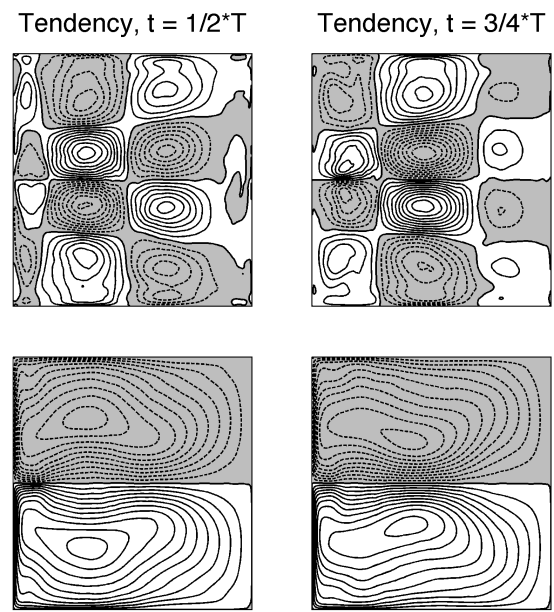

Figure 5-18: Tendency terms and corresponding effect on the mean baroclinic circulation induced by the $1 \times 2$ barotropic basin mode. The amplitude of the perturbation is set to match that of the perturbation baroclinic streamfunction filtered around the frequency of the $1 \times 2$ basin mode.

$1 \times 3$ basin mode, plotted at 4 different times, $t=0, \frac{T}{4}, \frac{T}{2}, \frac{3 T}{4}$ during the corresponding basin mode period $T$. The tendency terms illustrate how the mean interface displacement $\bar{\tau}(x, y)$ is advected by the respective basin mode. In addition, the re- 
I) Filtered baroclinic HEOF

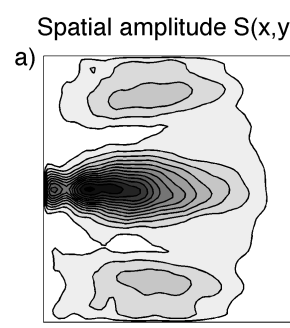

b)
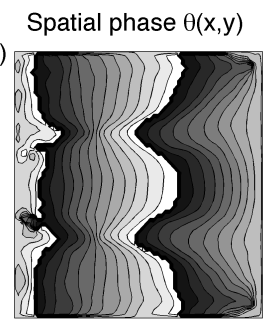
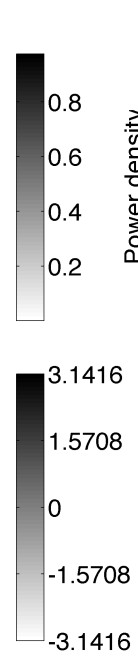
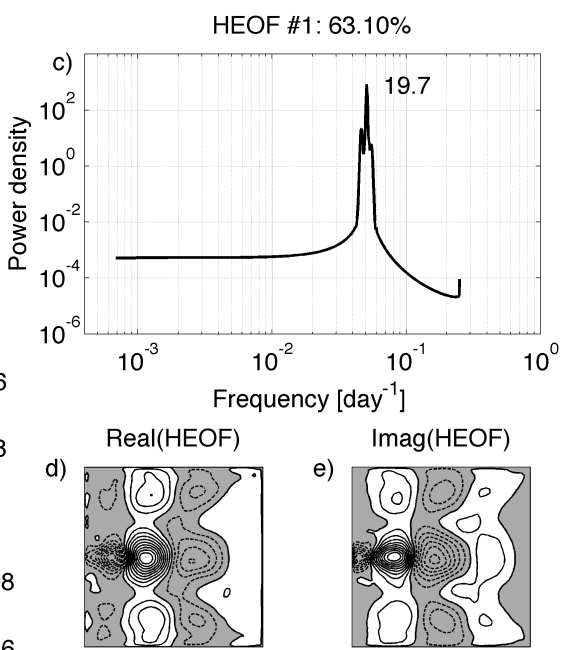

II) Perturbation flow

Spatial amplitude $S(x, y)$
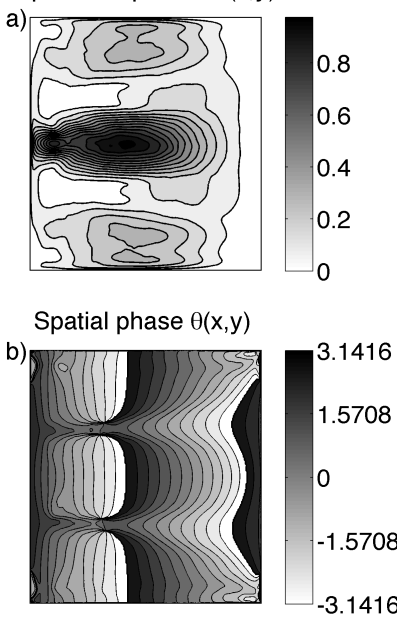

Figure 5-19: A) First HEOF mode of the baroclinic streamfunction in the weak bottom friction case $\left(r=10^{-9} \mathrm{~s}^{-2}\right)$ filtered around the period of the 2nd most dominant $1 \times 3$ barotropic basin mode, $T_{1 \times 3}=18.60$ days. B) Spatial amplitude and phase function for the baroclinic perturbation circulation driven by the basin mode.

Mean baroclinic $\Psi_{\mathrm{BC}}$
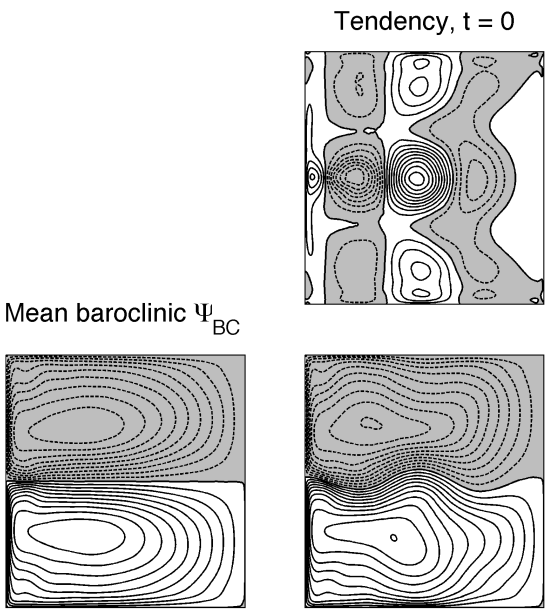
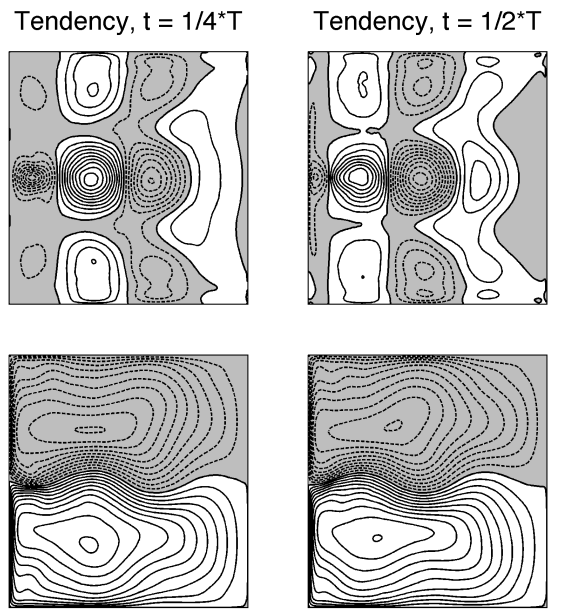
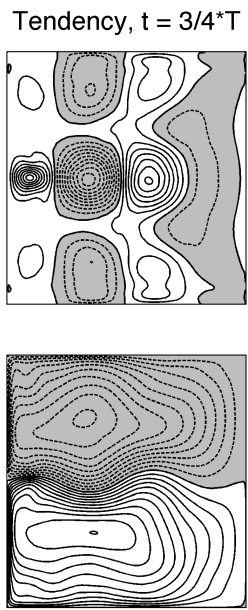

Figure 5-20: Tendency terms and corresponding effect on the mean baroclinic circulation induced by the $1 \times 3$ barotropic basin mode. The amplitude of the perturbation is set to match that of the perturbation baroclinic streamfunction filtered around the frequency of the $1 \times 3$ basin mode.

sulting perturbation flow $\tau^{\prime}(x, y, t)$ is added to the time-mean flow $\bar{\tau}(x, y)$, with an amplitude set to match approximatively that of the filtered baroclinic streamfunction around the corresponding basin mode period. It can be seen that the $1 \times 2$ basin 
mode drives variability consisting of large-scale gyre undulations, where the centers of the gyres undergo north-south and east-west excursions, while the mid-latitude jet changes its zonal extension and intensity. The $1 \times 3$ basin mode drives on the other hand variability consisting also of large-scale gyre undulations, but associated mainly with meandering of the midlatitude jet.

\subsection{Basin modes and the recirculations}

In the calculations analyzed so far, two different values for the bottom friction differing by a factor of 100 were used. It was determined that when the weak bottom friction is applied and the basin modes are less damped out, the recirculations are smaller and slightly weaker than when the large bottom drag is applied. One possible explanation for this behavior is that the basin modes have an adverse effect on the inertial recirculations.

The goal of this section is to verify this hypothesis. On the one hand we will establish the relationship between the strength of the recirculations and the applied bottom drag, which on its turn controls the degree to which the system variability is dominated by basin modes. We will also examine the vorticity balance for the recirculation gyres in order to assess the effect of the basin modes.

\subsubsection{Dependence of the inertial recirculations on bottom friction - spatially uniform case}

In order to obtain a better picture of how the recirculations depend on the intensity of the basin modes, it could be helpful to explore a larger range of bottom friction coefficients, in addition to the two values considered so far.

A series of eleven 30-year long calculations (after spin-up) were carried out where a spatially uniform bottom friction coefficient, varied from $r=5 \times 10^{-6} s^{-1}$ to $r=$ $1 \times 10^{-11} s^{-1}$, was applied. All other parameters, including the amplitude of the thermal forcing, the interface relaxation timescale and the eddy viscosity, were held 


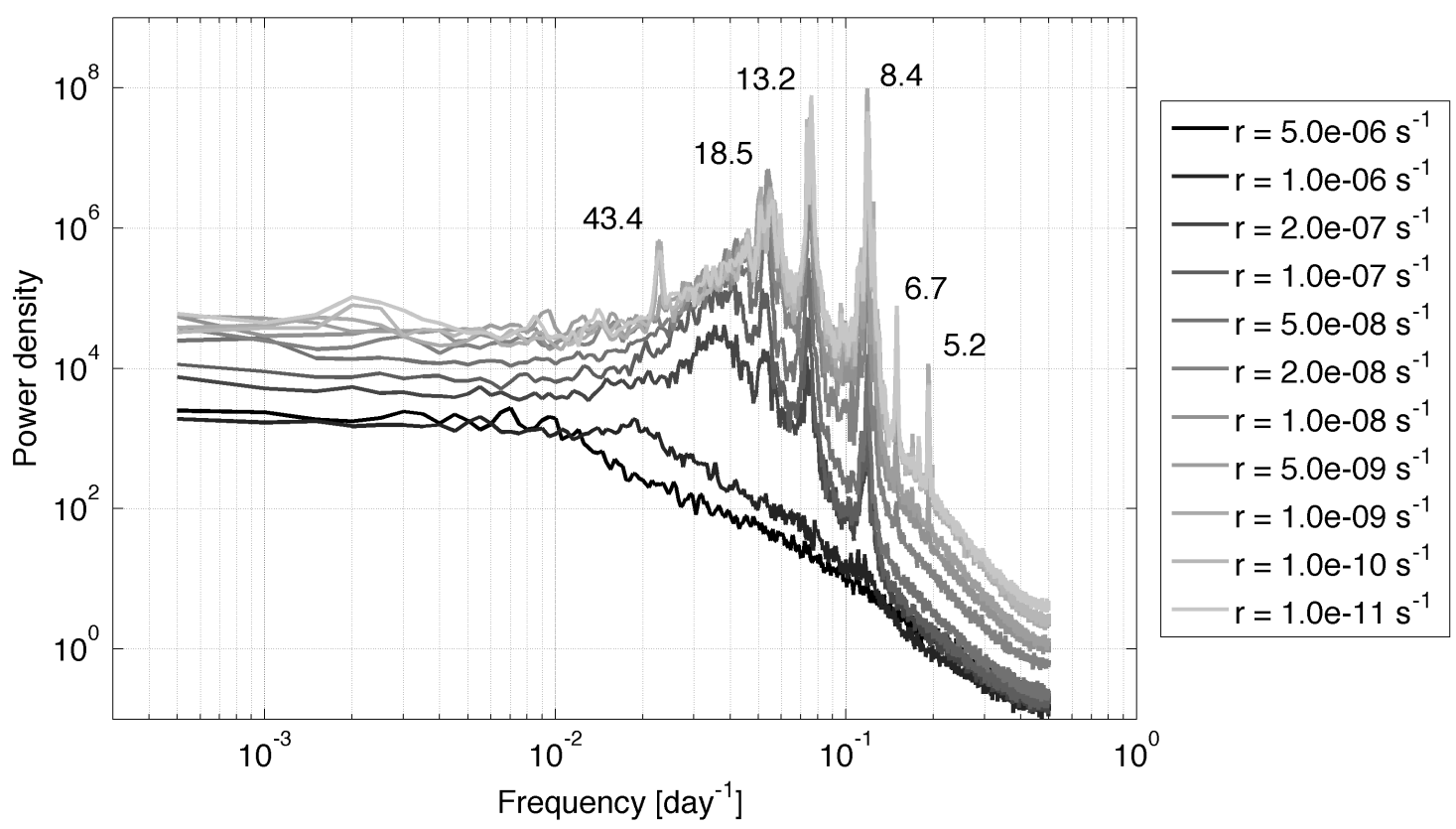

Figure 5-21: Power density spectra of the instantaneous barotropic streamfunction for a series of calculations, where a spatially uniform bottom friction with magnitude varying from $r=5 \times 10^{-6} s^{-1}$ to $r=1 \times 10^{-11} s^{-1}$ is applied. The period, in days, for the major spectral peaks is indicated.

the same. It is only for the two cases with largest bottom friction $r=1-5 \times 10^{-6} s^{-1}$ that the corresponding Stommel boundary layer is larger than the Munk boundary layer and is thus expected to play an active role in the boundary dynamics. For all other cases, the Stommel layer is much smaller than the Munk layer and is essentially not resolved by the grid. In terms of frictional spin-down timescales, the range covered is from approximatively 2 days for the largest bottom friction, to over 3000 years for the smallest bottom friction.

The power density spectra of the instantaneous barotropic streamfunction for the series of eleven calculations are plotted in Figure 5-21. Increasing the bottom friction has the effect of decreasing the overall barotropic variability in the system, as indicated by the progressively lower laying spectra. In addition, the stronger the applied bottom drag, the smaller the amount of variance contained in the highfrequency peaks. From all the spectral peaks present, three have been identified by 
HEOF analysis as being due to the resonance of barotropic Rossby basin modes. The 8.4 days peak corresponds to the gravest and fastest $1 \times 1$ basin mode. The 13.2 and 18.5 days peaks result mainly from the presence of a $1 \times 2$ and $1 \times 3$ basin mode respectively, although other basin modes with different spatial structure but the same or nearly the same periods might contribute as well. The basin mode peaks are present in all calculations, but the two using the largest bottom friction coefficients. There is an indication that, as the bottom friction is increased it is the gravest and fastest mode that is affected the most, while the higher order modes experience less damping. This is consistent with the fact the bottom drag in the presence of nonlinear advection tends to damp most the largest scales.

In addition to the basin mode spectral peaks, there are other high-frequency peaks present as well, in particular for the calculations with the four weakest bottom friction coefficients. There is some barotropic variability at periods 5-6 days that are shorter than that of the gravest basin mode $T_{1 \times 1}=8.3$ days. There is also some barotropic variability at periods of 43.4 days that is not accounted for by a high order basin mode. A HEOF analysis of the pass-filtered barotropic streamfunction around this period, shows that the variability at this timescale is confined to the western boundary region.

The time-mean barotropic circulation for the series of calculations with varying bottom drag is shown in Figure 5-22. As the bottom friction coefficient is decreased, a basin-scale time-mean circulation emerges. For bottom friction smaller than $r<$ $10^{-9} s^{-1}$, or equivalently frictional spin-down times larger that 30 years, the timemean circulation consists of a 4-gyre flow. This flow is driven, as already discussed, by the $1 \times 2$ Rossby basin mode that dominates the system variability.

Exploring the wider range of frictional coefficients reveals that for some moderate values for the frictional coefficient, in particular $r=5-10 \times 10^{-9} s^{-1}$, or equivalently frictional spin-down timescale on the order of 3-6 years, the time-mean circulation consists rather of a 6-gyre flow. Based on the analysis carried earlier on rectification of mean flow by the basin modes, we can deduce that the 6-gyre time-mean circulation 


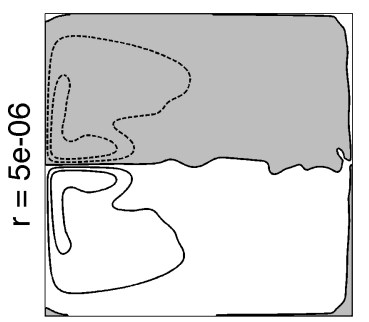

$\min =-0.1397, \max =0.1392$

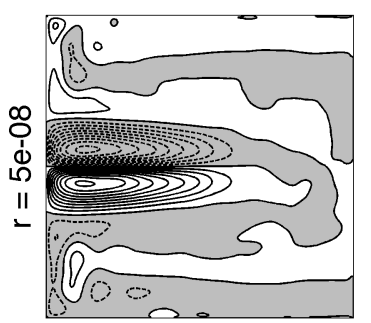

$\min =-0.4640, \max =0.4595$

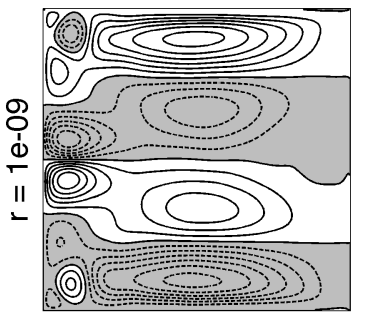

$\min =-0.3201, \max =0.3233$

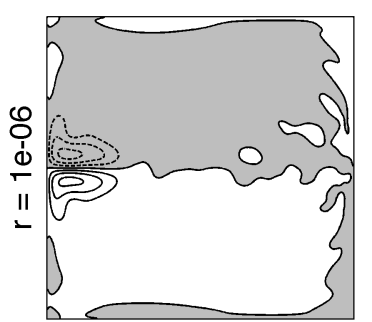

$\min =-0.1675, \max =0.1647$

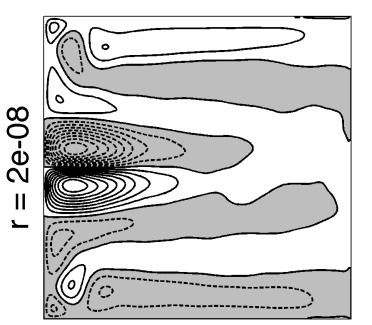

$\min =-0.4370, \max =0.4437$

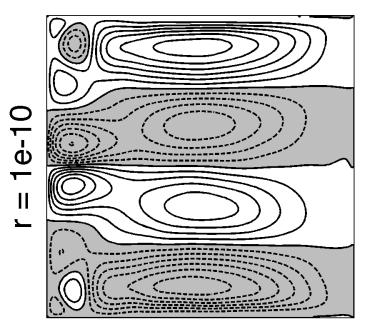

$\min =-0.3308, \max =0.3292$

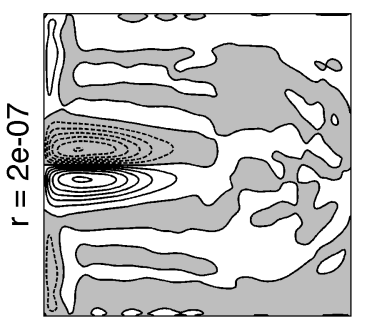

$\min =-0.3023, \max =0.3076$
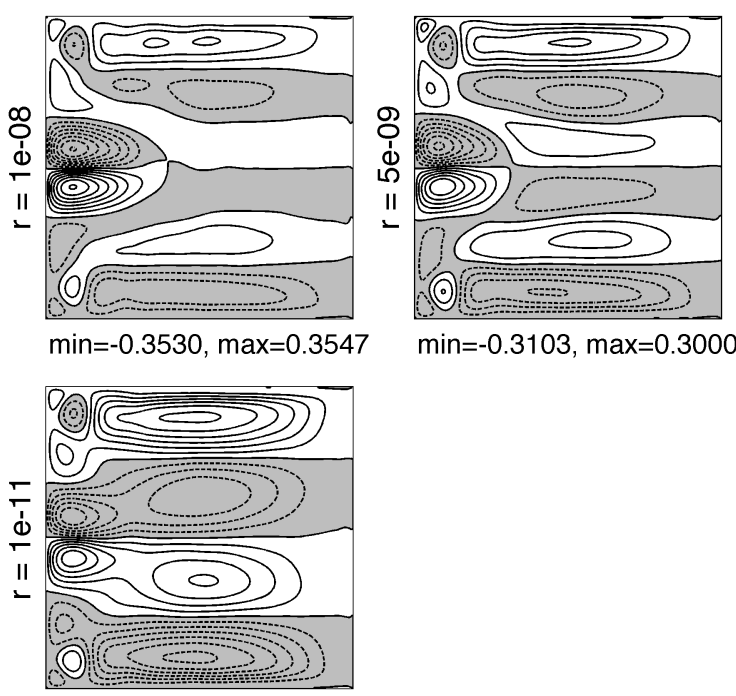

$\min =-0.3119, \max =0.3113$

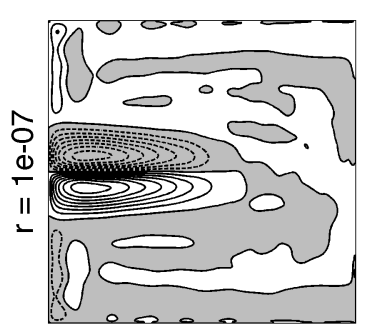

$\min =-0.3103, \max =0.3000$

Figure 5-22: Time-mean barotropic circulation for a series of calculations, where a spatially uniform bottom friction with magnitude varying from $r=5 \times 10^{-6} \mathrm{~s}^{-1}$ to $r=1 \times 10^{-11} s^{-1}$ is applied. The streamfunction is scaled by the maximum linear baroclinic streamfunction $\tau^{(l i n)}=\frac{g^{\prime} h_{0}}{f_{0}}\left(1-e^{-\frac{1}{\delta_{T}}}\right)=0.25 \times 10^{6} \mathrm{~m}^{2} \mathrm{~s}^{-1}$. The contour interval on all plots is 0.05 .

is driven by the $1 \times 3$ Rossby basin mode (see Figure $5-8$ ). That suggests that at these moderate values for the bottom friction it is the higher order $1 \times 3$ basin mode that dominates the system variability, instead of the $1 \times 2$ basin mode. This conclusion is consistent with our earlier findings from the power spectra that the higher order basin modes are less damped by the bottom friction.

Finally, it can be seen that the circulation saturates in the limit of weak bottom friction. Although the frictional coefficient is changed by two orders of magnitude from $r=10^{-9} s^{-1}$ to $r=10^{-11} s^{-1}$, or equivalently for the frictional spin-down timescale from 30 to 3000 years, the time-mean circulation pattern and maximum 
transports barely change.

Varying the value of the bottom friction coefficient has a major effect not only on the basin-scale time-mean barotropic circulation, but also on the inertial recirculations. As a guideline for the size of the recirculations, the streamline where the value of the time-mean barotropic streamfunction decreases to half of its maximum value is chosen. This is a rather tight estimate, but has the advantage that it can be used for all calculations considered here, including the weak bottom friction cases, where the recirculations and the interior flow become on the same order and are difficult to separate. When applying this definition, recirculation gyres can be isolated in all calculations, but the one using the largest bottom friction coefficient.

In Figure 5-23 some characteristics of the recirculations such as strength, measured by the maximum/minimum of the streamfunction, zonal extent and position of the center are plotted as a function of the bottom friction coefficient. For reference, the corresponding frictional spin-down timescale $1 / r$ is indicated as well. As the bottom friction is decreased from the largest value used here, the strength of the recirculations and their zonal extent increase. This is an anticipated behavior given that the bottom drag acts to damp the barotropic component of the circulation. Thus, the smaller the amount of bottom friction in the system, the stronger is expected to be the resulting time-mean barotropic circulation.

However, both the strength and zonal extent of the recirculation gyres reach a maximum around $r=5 \times 10^{-8} s^{-1}$, or equivalently for frictional spin-down timescale of approximatively 230 days. After that, despite the fact that the bottom friction is further decreased, the recirculation gyres decrease in strength and size, and eventually saturate. As seen earlier in this section from examining the power spectra of the instantaneous barotropic streamfunction (Figure 5-21) and the time-mean barotropic circulation patterns (Figure 5-22), it is exactly for values of the bottom drag of $r=5 \times 10^{-8} s^{-1}$ and smaller that the basin modes start to dominate the system variability. This supports the idea of a connection between the recirculation gyres intensity and the basin modes - the more the system variability is dominated by the 
a)
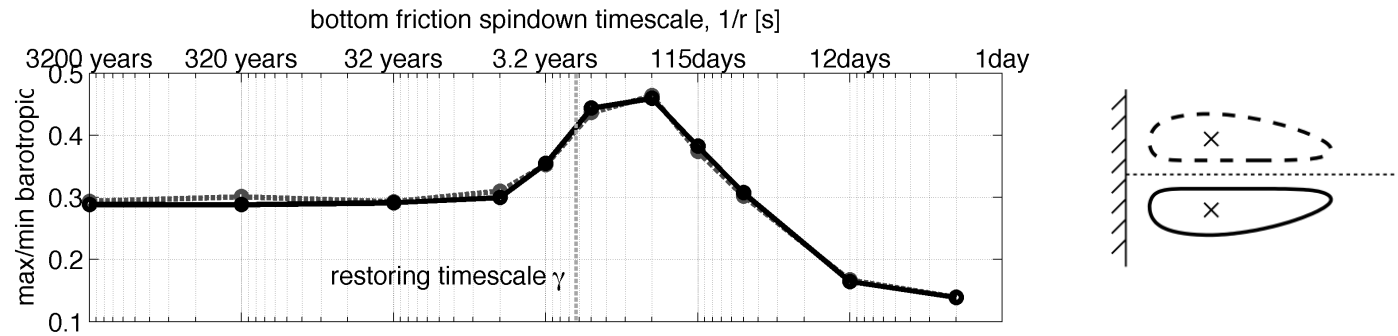

b)
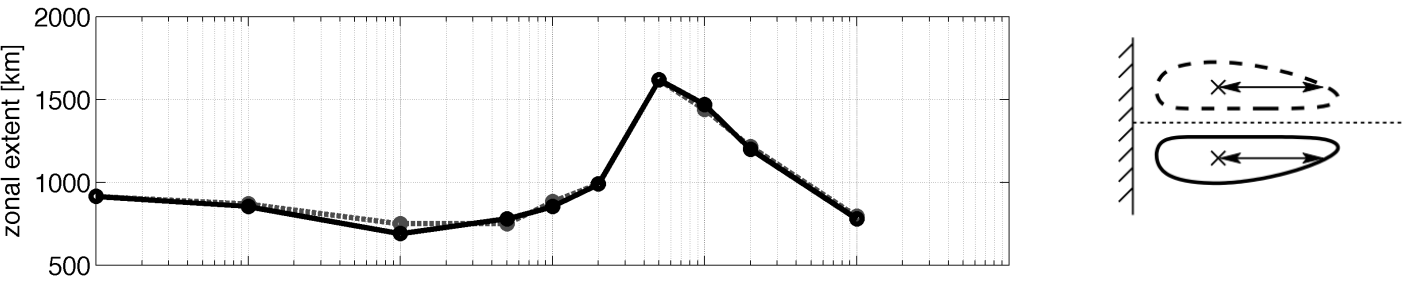

c)
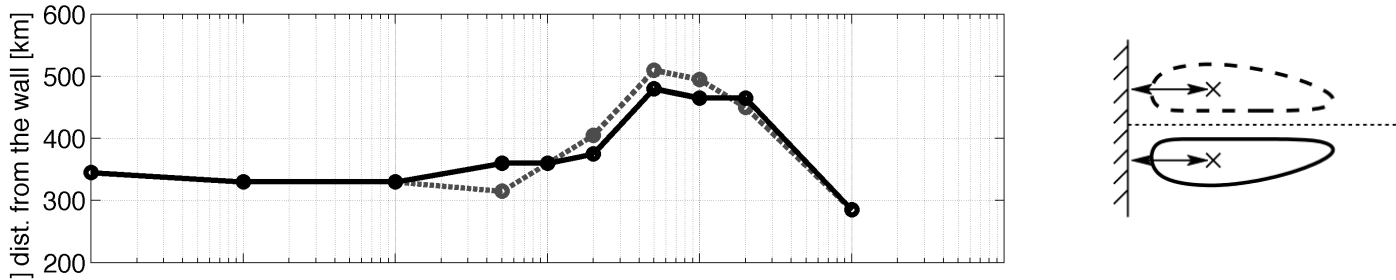

d)
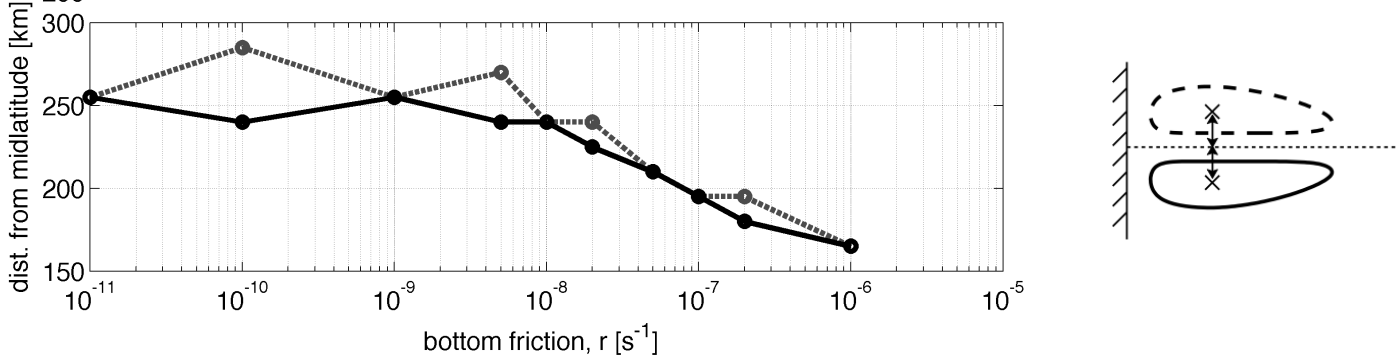

Figure 5-23: Characteristics of the barotropic inertial recirculations for a series of calculations, where a spatially uniform bottom friction with magnitude varying from $r=5 \times 10^{-6} s^{-1}$ to $r=1 \times 10^{-11} s^{-1}$ is applied. The bottom friction is also given in terms of the frictional spin-down timescale $1 / r$ on the top x-axis. The panels show: a) absolute value of the maximum/minimum time-mean barotropic streamfunction; b) zonal extent of the recirculation; c) and d) position of the recirculation center from the western wall and the mid-latitude, respectively. Solid/dashed line is for the southern/northern recirculation.

basin modes, the less intense and the more spatially confined are the recirculation gyres.

Finally, it can be seen that the magnitude of the bottom friction affects not only the strength and size of the recirculation gyres, but also their spatial structure. Plotted in Figure 5-23(c-d) is the position of the center of each recirculation, with the 
center being defined as the location where the time-mean barotropic streamfunction reaches its maximum/minimum value. As the bottom friction is decreased, the centers of the recirculations retract toward the western wall and shift away from the mid-latitude. The result is zonally elongated recirculations, when they are at their maximum intensity, and much more round-shaped and confined to west recirculations, when the basin modes start intervening.

\subsubsection{Dependence of the inertial recirculations on bottom friction - spatially variable case}

Another way to verify the link between the intensity of the inertial recirculations and the basin modes is to apply a spatially variable bottom drag. The idea behind employing a spatially drag coefficient is that it introduces a spatial inhomogeneity in the model that interferes with the basin modes.

A series of experiments was performed, where the bottom friction coefficient is uniform in the meridional direction, but varies zonally, switching from $r=10^{-9} s^{-1}$ to $r=10^{-7} s^{-1}$ from west to east. These are exactly the same values used in the uniformly weak and uniformly strong bottom friction cases examined in details initially. The transition between the two values for the bottom drag happens through a linear increase of the friction coefficient over a distance of $600 \mathrm{~km}$, with the transition zone being pushed further and further east (Figure 5-24). By subjecting a gradually smaller portion of the basin to the strong bottom drag, the basin modes are allowed to become more dominant, while locally the recirculation gyres are exposed to the same weak bottom friction. These experiments are designed therefore to further test the hypothesis that it is the basin modes that affect adversely the recirculations, and not some other local effect.

In Figure 5-24 the resulting time-mean barotropic circulation averaged over 30 years of time integration (after spin-up) is shown. For reference, the frictional coefficient profiles in the zonal direction applied in each case are plotted as well. The 

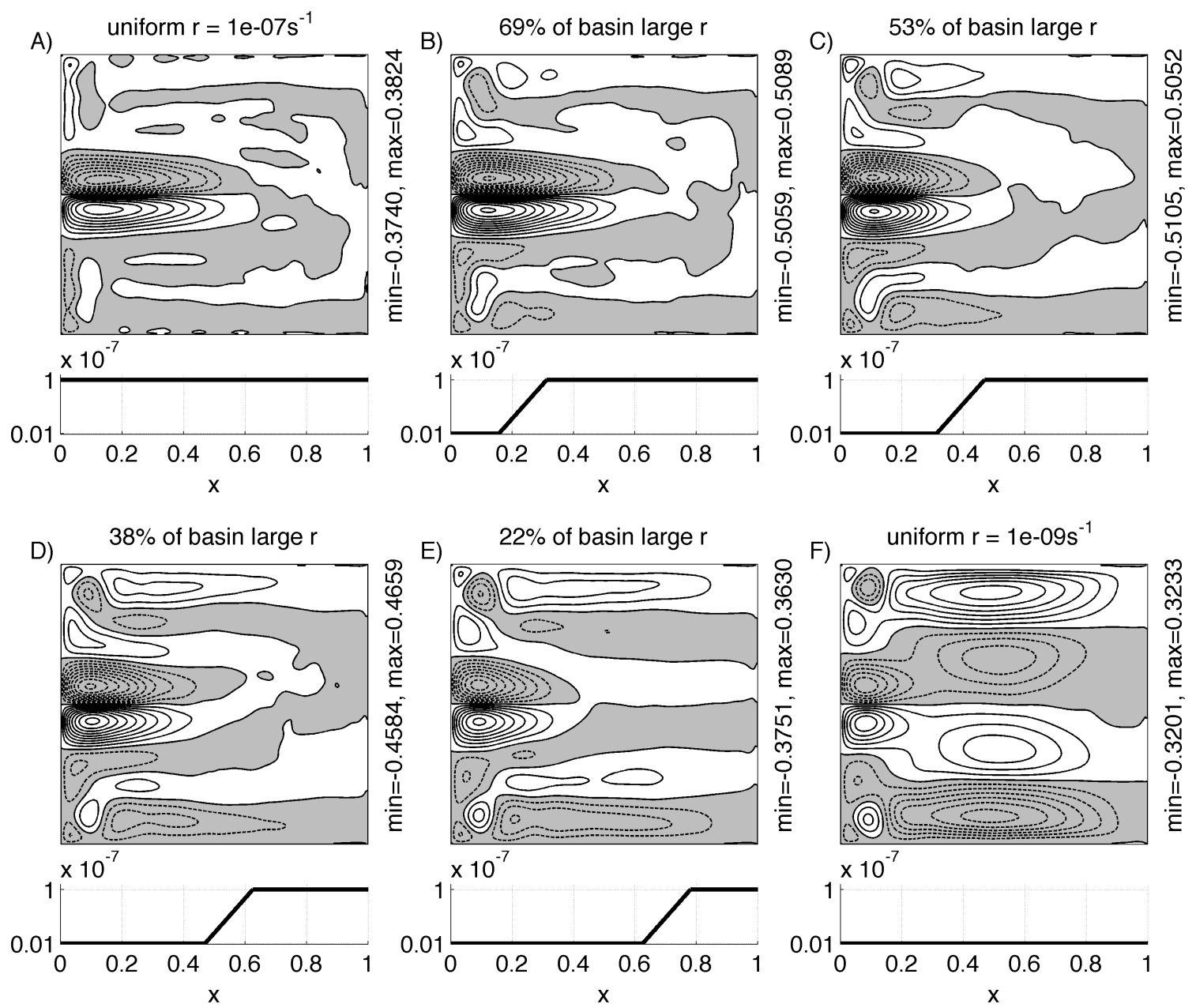

Figure 5-24: Time-mean barotropic circulation for a series of calculations where spatially varying bottom friction is applied. The bottom friction changes zonally from $r=10^{-9} s^{-1}$ to $r=10^{-7} s^{-1}$, as shown on the lower plot of each panel. The percentage of basin area subject to the strong bottom friction is indicated in the title. The streamfunction is scaled by the maximum linear regime baroclinic transport $\tau^{(l i n)}=\frac{g^{\prime} h_{0}}{f_{0}}\left(1-e^{-\frac{1}{\delta_{T}}}\right)=0.25 \times 10^{6} m^{2} s^{-1}$. The contour interval on all plots is 0.05 .

transition zone between the weak and strong bottom friction regions is pushed gradually east - it starts for the different cases at 600,1200, 1800 and $2400 \mathrm{~km}$ respectively from the western wall (the size of the square basin is $L=3840 \mathrm{~km}$ ). On the plots, it is the percentage of the basin area subject to the uniformly strong bottom drag that is indicated in order to differentiate between the cases. Also for completeness, the time-mean circulation for the uniformly weak and uniformly strong bottom cases 
are added as well as limit cases. The power density spectra of the instantaneous barotropic streamfunction for all cases is given in Figure 5-25, while a summary of some characteristics of the recirculations, such as strength and zonal extent and, are plotted in Figure 5-26.

As smaller portion of the domain is subject to the strong bottom friction, the basin modes become more dominant, as illustrated by the larger magnitude high-frequency spectral peaks (Figure 5-25). Simultaneously with that, the size of the recirculation gyres in the time-mean circulation decreases (Figure 5-26(b)). The zonal extent of the recirculations seems unrelated to the spatial structure of the applied bottom friction, in the sense that it does not seem to be determined by the position of the transition zone (Figure 5-24). This confirms the idea that it is the presence of basin modes that affects adversely the recirculation gyres, and not the local damping by bottom drag.

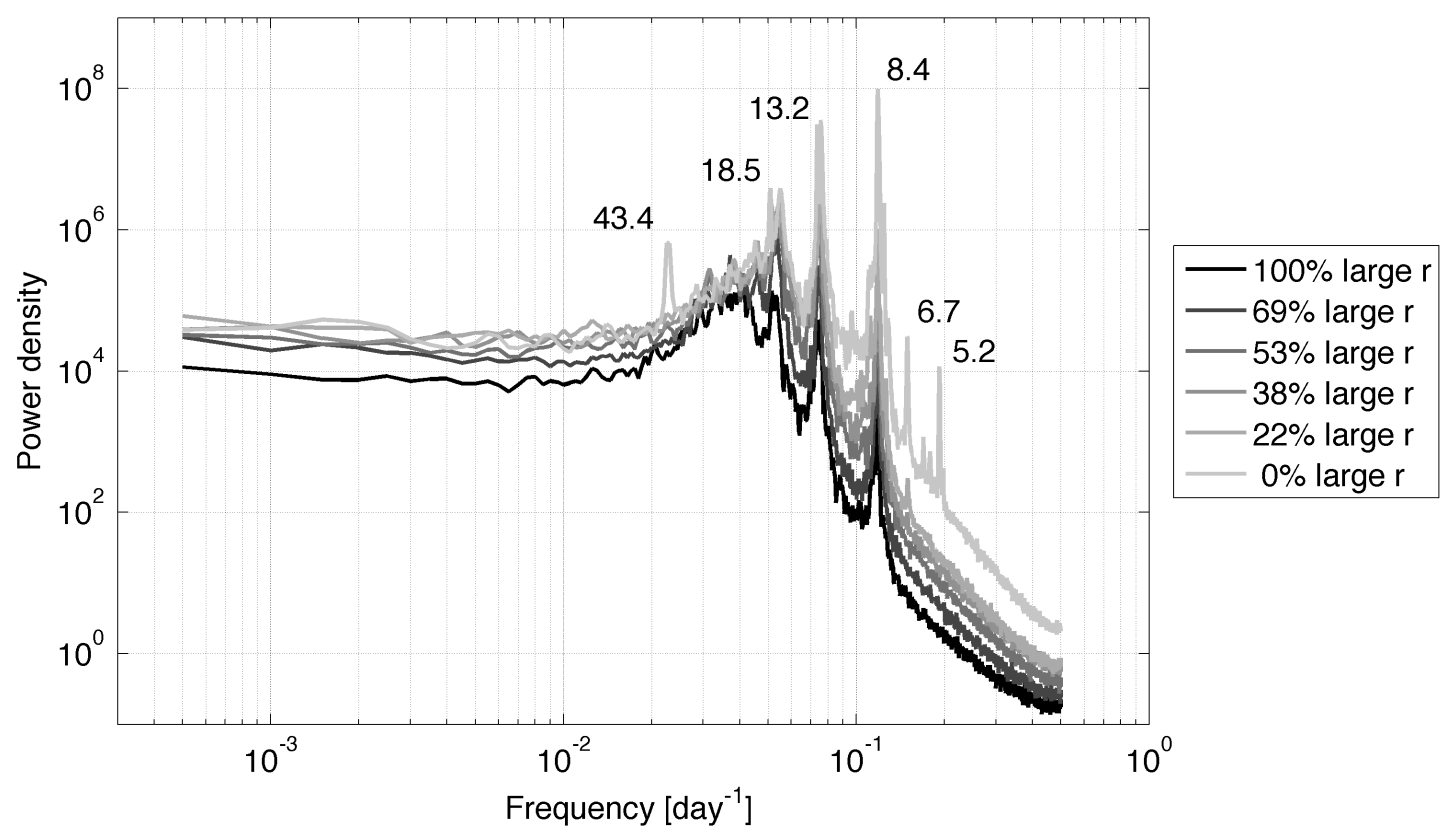

Figure 5-25: Power density spectra of the instantaneous barotropic streamfunction for a series of calculations, where a spatially varying bottom friction is applied. The bottom friction changes zonally from $r=10^{-9} s^{-1}$ to $r=10^{-7} s^{-1}$, with the percentage of basin area subject to the strong bottom friction used in the legend. The period, in days, for the major spectral peaks is indicated. 
Concerning the strength of the recirculation gyres as measured by the minimum/maximum of the the time-mean barotropic streamfunction, the picture is a little more complex. In general, as a larger part of the basin becomes subject to the strong bottom drag that damps the basin modes, the strength of the recirculation gyres increases, in accord with the hypothesis that the basin modes have a negative effect on them (Figure 5-26(a)). However, it can be seen also that the recirculations are actually slightly stronger when a thin band of weak bottom friction is present next to the western wall, compared to the case when uniformly strong bottom friction is applied everywhere. Although, the basin modes are more damped in the later case, this does not translate into more intense recirculations. This implies that, at least in what concerns the strength of the inertial recirculations, local processes taking place next to the western wall matter as well, and it is not simply a consequence of the importance of the basin modes.

a)

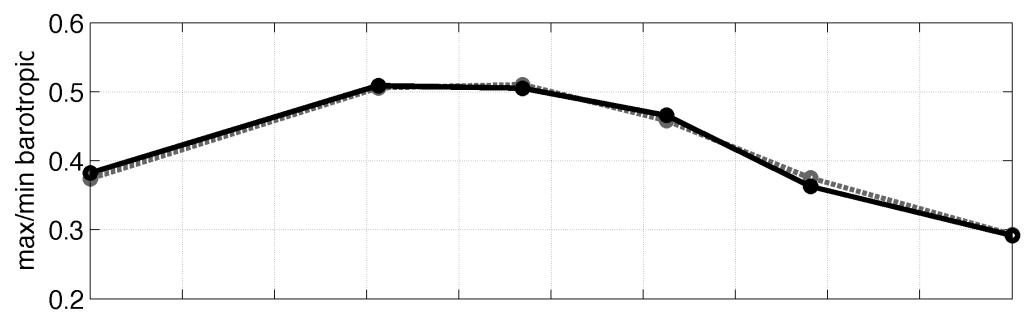

b)

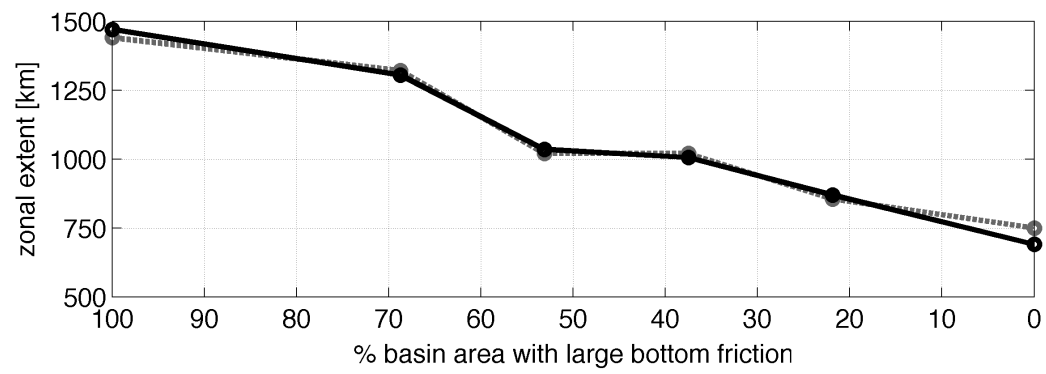

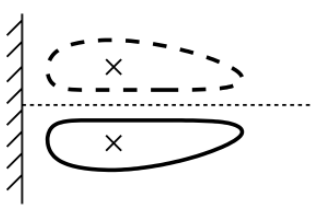

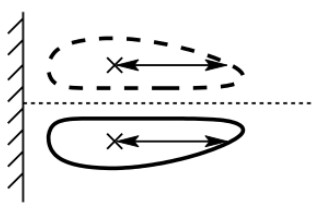

Figure 5-26: Characteristics of the barotropic inertial recirculations for a series of calculations, where a spatially varying bottom friction is applied. The bottom friction changes zonally from $r=10^{-9} s^{-1}$ to $r=10^{-7} s^{-1}$, with the percentage of basin area subject to the strong bottom friction used as the x-axis. The panels show: a) absolute value of the maximum/minimum time-mean barotropic streamfunction; b) zonal extent of the recirculation. Solid/dashed line is for the southern/northern recirculation. 
Finally, there are also some changes in the basin-scale time-mean circulation. As gradually smaller portion of the basin is subject to the strong bottom friction and the basin modes become more intense, a large-scale time-mean barotropic circulation emerges (Figure 5-24). The circulation is confined to the part of the basin exposed to the weak bottom friction and is of the form of a 6- and 4-gyre flow, with the outer gyres situated right next to southern and northern walls being the strongest. The time-mean flow is driven by the basin modes, as shown in the analysis on rectification carried previously.

\subsubsection{Driving mechanism for the inertial recirculation gyres}

In order to determine what is the driving mechanism for the inertial recirculations, similar tools as in the steady regime, such as local and integrated vorticity budgets of the barotropic vorticity, are used. However, when considering the vorticity budget of a time-dependent circulation, the additional contributions from the eddy fluxes need to be included.

\section{Vorticity budget in the time-dependent regime}

Let introduce the following decomposition into mean and eddy part for a given generic variable $\psi(x, y, t)$ in the time-dependent regime,

$$
\bar{\psi}=\frac{1}{\Delta T} \int_{t}^{t+\Delta T} \psi(x, y, t) d t, \quad \psi^{\prime}=\psi-\bar{\psi}
$$

The mean part $\bar{\psi}$ represents the long time-mean equilibrium reached by the system, where all variability on timescales shorter than $\Delta T$ has been averaged out, and only variability on timescales longer than $\Delta T$ remains. The eddy part $\psi^{\prime}$ represents the perturbations from the long time-mean equilibrium. For the calculations considered here, this can include both mesoscale eddy variability and basin mode variability.

Applying the time-averaging operation to the barotropic potential vorticity equa- 
tion (3.25), leads to the following balance for the time-mean barotropic vorticity

$$
\begin{aligned}
\underbrace{\frac{\partial}{\partial t} \nabla^{2} \bar{\phi}}_{\text {time residue }}= & -\underbrace{\left[\frac{\partial \bar{\phi}}{\partial x}+\delta_{I}^{2}\left(\overline{J\left(\phi, q_{\phi}\right)}+\delta(1-\delta) \overline{J\left(\tau, q_{\tau}\right)}\right)\right]}_{\text {total Jacobian, } J_{\phi}} \\
& +\underbrace{\underbrace{\delta_{M}^{3} \nabla^{4} \bar{\phi}}_{\text {bottom friction }}}_{\text {lateral dissipation }}-\underbrace{\delta_{S}(1-\delta) \nabla^{2}(\bar{\phi}-\delta \bar{\tau})} .
\end{aligned}
$$

On the left-hand side of the equation is the residual time rate of change of the vorticity, occurring on timescales long compared to the averaging interval. This term is expected to be approximatively zero, if the averaging is taken over a sufficiently long time interval, so that the system is close to a statistically steady equilibrium. In this case, at each point in the basin a local balance between bottom friction, lateral dissipation and advection, marked as $J_{\phi}$, is expected. The total Jacobian $J_{\phi}$, the only term containing nonlinearity, can be further decomposed into several parts - advection of planetary vorticity, mean Jacobian and eddy Jacobian, the last two being defined using respectively the mean and eddy streamfunction

$$
\begin{aligned}
& J_{\phi}=\underbrace{\frac{\partial \bar{\phi}}{\partial x}}_{\text {mean Jacobian }}+\underbrace{\delta_{I}^{2}\left[J\left(\bar{\phi}, \nabla^{2} \bar{\phi}\right)+\delta(1-\delta) J\left(\bar{\tau}, \nabla^{2} \bar{\tau}\right)\right]} \\
& \text { planetary } \\
& \text { (mean fluxes divergence) } \\
& +\underbrace{\delta_{I}^{2}\left[\overline{J\left(\phi^{\prime}, \nabla^{2} \phi^{\prime}\right)}+\delta(1-\delta) \overline{J\left(\tau^{\prime}, \nabla^{2} \tau^{\prime}\right)}\right]}_{\text {eddy Jacobian }} .
\end{aligned}
$$

(eddy flux divergence)

Each of the Jacobian terms represents a divergence of a vorticity flux. For example, $J\left(\bar{\phi}, \nabla^{2} \bar{\phi}\right)$ and $\overline{J\left(\phi^{\prime}, \nabla^{2} \phi^{\prime}\right)}$ stand for the divergence of respectively the mean flux of mean barotropic vorticity and the eddy flux of eddy barotropic vorticity, i.e.

$$
J\left(\bar{\phi}, \nabla^{2} \bar{\phi}\right)=\nabla \cdot\left(\overline{\mathbf{u}}_{\phi} \nabla^{2} \bar{\phi}\right) \quad \text { and } \quad \overline{J\left(\phi^{\prime}, \nabla^{2} \phi^{\prime}\right)}=\nabla \cdot \overline{\left(\mathbf{u}_{\phi}^{\prime} \nabla^{2} \phi^{\prime}\right)}
$$

Similarly to what was done in the steady regime, the entire time-averaged barotropic 
equation (5.18) can be written in a flux divergence form (Fox-Kemper, 2003) and integrated over a region $C_{\phi}$ enclosed by a time-mean barotropic streamline. Assuming statistically steady equilibrium and excluding all contributions from fluxes proportional to the mean barotropic velocity, the integrated barotropic vorticity budget becomes

$$
\begin{aligned}
\oint_{C_{\phi}}\left[-\delta_{I}^{2} \delta(1-\delta) \overline{\mathbf{u}}_{\tau} \nabla^{2} \bar{\tau}\right. & -\delta_{I}^{2} \overline{\mathbf{u}_{\phi}^{\prime} \nabla^{2} \phi^{\prime}}-\delta_{I}^{2} \delta(1-\delta) \overline{\mathbf{u}_{\tau}^{\prime} \nabla^{2} \tau^{\prime}} \\
& \left.+\delta_{M}^{3} \nabla\left(\nabla^{2} \bar{\phi}\right)-\delta_{S}(1-\delta) \nabla(\bar{\phi}-\delta \bar{\tau})\right] \cdot \hat{\mathbf{n}} d l=0
\end{aligned}
$$

Because the circulation is generated from a thermal-only forcing, barotropic potential vorticity is neither added or removed from the system, but merely rearranged. This is accomplished by 1) the mean advection by the baroclinic flow, 2) the eddy fluxes (barotropic or baroclinic), and 3) the frictional fluxes due to lateral or bottom friction. Therefore, for each area enclosed by a mean barotropic streamline the cross-streamline fluxes resulting from these terms have to balance out.

If the special case of the streamline coinciding with the basin boundary is considered, then the integral condition (5.21) reduces to

$$
\oint_{C} \delta_{M}^{3} \nabla\left(\nabla^{2} \bar{\phi}\right) \cdot \hat{\mathbf{n}} d l=0
$$

Because of the no-normal flow and the no-slip conditions, both the mean and eddy vorticity fluxes vanish at the walls. Thus, the role of all advective fluxes, mean or eddy, when no-slip conditions are applied is limited to redistribution of the vorticity within the basin. In addition, due to the lack of barotropic vorticity input from external forcing for a thermally-forced ocean, there is no net frictional vorticity flux through the basin boundaries either. This is very different from a single gyre wind-driven circulation, where there is a net barotropic vorticity input from external forcing. Then, vorticity needs to be transported by different means from the interior, where it is added by the wind, to the boundaries, where it can be removed by the lateral 
friction (Fox-Kemper, 2003).

Next, the barotropic vorticity budget is examined for the cases of weak and strong bottom friction with the goal in mind to assess the effect of the basin modes on the inertial recirculations. For the purpose of these calculations, a time-average over 120 years is considered and a statistically steady equilibrium budget is expected.

\section{Strong bottom friction case - barotropic vorticity budget}

In Figure 5-27 the local barotropic vorticity budget for the strong bottom friction case is plotted. All terms are shown with exactly the same sign convention as in equation (5.18). It can be seen that the main vorticity balance in the basin is between convergences and divergences of vorticity flux due to advection $\left(-J_{\phi}\right)$ and bottom friction. The lateral diffusion contributes as well, mainly within the western boundary layer and along the mid-latitude jet, but is otherwise weak in the remaining of the basin. The time residue is orders of magnitude smaller than all the other terms, consistent with a system close to a statistically steady state.

In Figure 5-28 the total Jacobian is decomposed into planetary vorticity advection and mean and eddy flux convergences*. The convergence of the planetary vorticity flux $-\bar{\phi} \hat{\mathbf{x}}$ is aligned with the mean meridional barotropic velocity $\bar{v}_{\phi}$. Because there is no large-scale time-mean barotropic circulation, this term is confined to the recirculations and the western boundary layer. The mean and eddy fluxes both include contributions from the baroclinic and barotropic components of the circulation. Although not shown here, both these terms are dominated by the advection by the barotropic velocity of the barotropic relative vorticity $\nabla \cdot\left(\mathbf{u}_{\phi} \nabla^{2} \phi\right)$ (either mean or eddy). For the mean vorticity flux only, the baroclinic advection of baroclinic vorticity, $\nabla \cdot\left(\overline{\mathbf{u}}_{\tau} \nabla^{2} \bar{\tau}\right)$ matters as well, but mostly in the western boundary layer. All vorticity flux divergences due to the advective terms, either mean or eddy, are concentrated mostly in the western boundary layer and the recirculations.

${ }^{*}$ Note that all these terms are plotted with a sign opposite to that in equation (5.19), since it is $-J_{\phi}$ that appears in the barotropic vorticity budget (5.18). 

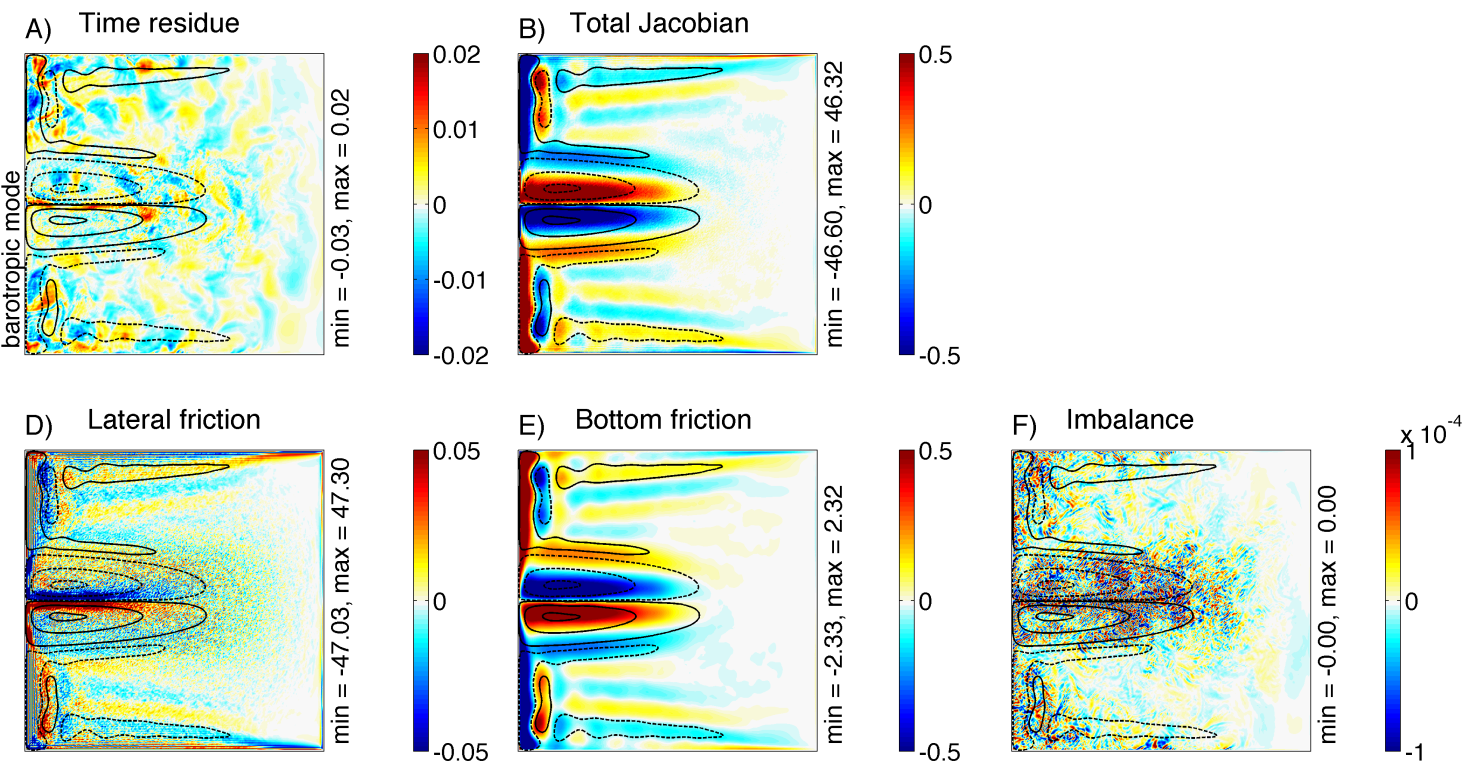

Figure 5-27: Barotropic potential vorticity budget for the case with strong bottom friction $\left(r=10^{-7} s^{-1}\right)$. All terms are as indicated in Eq. (5.18). The missing panel C is the thermal forcing. Selected contours of the time-mean barotropic streamfunction are overlaid in black.
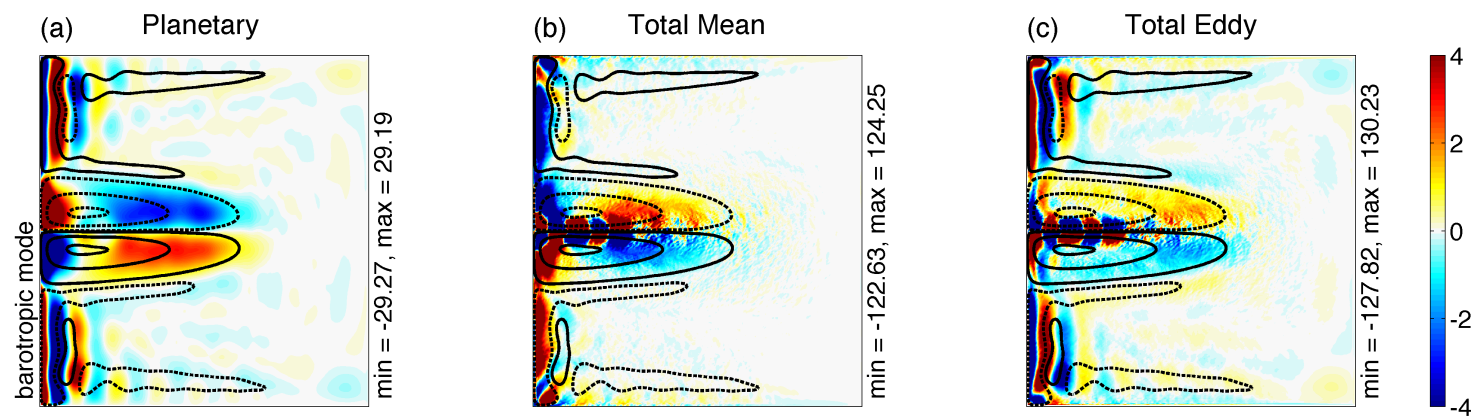

Figure 5-28: Decomposition of the total barotropic Jacobian $-J_{\phi}$, given in panel B) above, into: a), planetary vorticity advection, $-\nabla \cdot(\bar{\phi} \hat{\mathbf{x}}) ;$ b) mean Jacobian or mean vorticity flux convergence, $-\nabla \cdot\left(\overline{\mathbf{u}}_{\phi} \nabla^{2} \bar{\phi}+\delta(1-\delta) \overline{\mathbf{u}}_{\tau} \nabla^{2} \bar{\tau}\right)$; c) eddy Jacobian or eddy vorticity flux convergence, $-\nabla \cdot \overline{\left(\mathbf{u}_{\phi}^{\prime} \nabla^{2} \phi^{\prime}+\delta(1-\delta) \mathbf{u}_{\tau}^{\prime} \nabla^{2} \tau^{\prime}\right)}$.

In order to determine the cross-streamline vorticity fluxes, the barotropic vorticity equation is integrated over a series of regions bounded by mean barotropic streamlines all laying in the northern recirculation gyre. Each next streamline encloses a larger area region, starting from the recirculation center and expanding toward the outer 


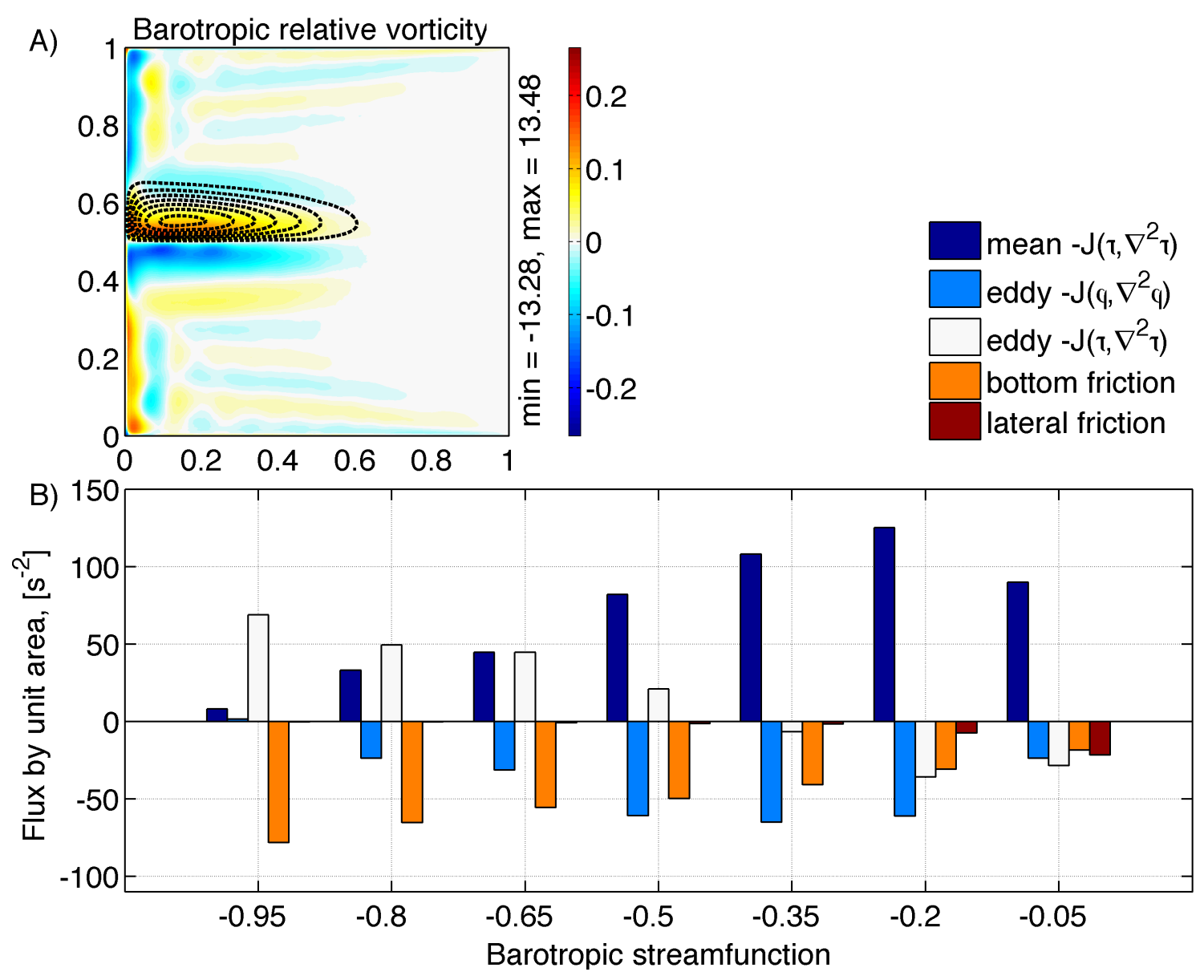

Figure 5-29: Barotropic potential vorticity budget for the case with strong bottom friction $\left(r=10^{-7} s^{-1}\right)$, integrated over a series of regions enclosed by mean barotropic streamlines. Panel A), mean barotropic relative vorticity field, scaled with the maximum planetary vorticity. Overlaid are contours of the mean barotropic streamfunction, delimiting the regions of integration, $\frac{\bar{\phi}}{|\min (\bar{\phi})|} \in[-0.05,-0.95]$. Panel B), integral of the vorticity flux divergence by unit area. The different terms are as indicated in Eq. (5.21).

edge, as shown in Figure 5-29. In order to be able to compare the fluxes out of these regions, the integrals of the vorticity flux divergences from equation (5.21) have been divided by the area of the region, i.e the flux by unit area is plotted. In addition, in Table 5.3 the details of the integrated vorticity balance over the region enclosed by the streamline, where $\bar{\phi}$ is equal to half of its minimum value, are given. Since the northern anticlockwise recirculation is characterized with positive mean 


\begin{tabular}{crrr}
\hline \hline Term & $\begin{array}{r}\text { Integral S } \\
\text { (recirc.) }\end{array}$ & $\begin{array}{r}\text { Integral W } \\
\text { (recirc.) }\end{array}$ & $\begin{array}{r}\text { Integral W } \\
\text { (interior) }\end{array}$ \\
\hline \hline Mean flux, $-J\left(\bar{\tau}, \nabla^{2} \bar{\tau}\right)$ & 100.00 & 100.00 & 100.00 \\
Eddy flux, $-\overline{J\left(\phi^{\prime}, \nabla^{2} \phi^{\prime}\right)}$ & -74.56 & -130.96 & 202.62 \\
Eddy flux, $-\overline{J\left(\tau^{\prime}, \nabla^{2} \tau^{\prime}\right)}$ & 25.46 & 16.46 & -300.21 \\
Thermal forcing & 0.00 & 0.00 & 0.00 \\
Lateral friction & -1.87 & -0.82 & -13.41 \\
Bottom friction & -60.84 & -0.47 & -18.48 \\
\hline Imbalance & -11.80 & -15.79 & -29.48 \\
(due to planetary) & -8.82 & -16.69 & 6.51 \\
(due to time residue) & -0.17 & -0.06 & -32.68 \\
\hline \hline Dimensional $-J\left(\bar{\tau}, \nabla^{2} \bar{\tau}\right)$ & 1554.90 & 815.24 & 3.87 \\
in units $\mathrm{cm}^{2} s^{-2}$ & \multirow{2}{*}{} & & \\
\hline \hline
\end{tabular}

Table 5.3: Barotropic potential vorticity balance integrated over the region enclosed by the mean barotropic streamline, where $\bar{\phi}$ is equal to half of its minimum value. The region of integration lays either in the northern recirculation gyre (recirc.) or in the interior gyre (interior), if present. S, stands for the case with strong bottom friction $\left(r=10^{-7} s^{-1}\right)$, W, for the case with weak bottom friction $\left(r=10^{-9} s^{-1}\right)$. All terms are as indicated in Eq.(5.21). The integral of the mean flux divergence is scaled to 100 , with the actual value given in the last row.

relative vorticity (Figure 5-29(A)), positive values of the integrated flux divergence are interpreted as flux into the region, while negative values as flux out of the region.

From the results listed in Table 5.3 it can be seen that the largest cross-streamline flux into the recirculation is due to the mean baroclinic advection, $\overline{\mathbf{u}}_{\tau} \nabla^{2} \bar{\tau}$. The magnitude of this term has been scaled to 100 for easier comparison later with the weak bottom friction case, with the actual value of the flux given as well for reference. Therefore, the recirculations are driven by the mean and eddy baroclinic fluxes, while the barotropic eddy flux, lateral diffusion and bottom drag all export vorticity out of the recirculations and act thus to damp them. It can be seen that the imbalance for the integrated vorticity budget is not insignificant. It is mostly due to the planetary vorticity advection integral. In general, this term should integrate to zero, but since a discretized version of the region enclosed by the streamline is used, an error is introduced. 
When the integrated vorticity budget is computed for a series of regions, covering progressively larger portion of the northern recirculation, it can be seen that the relative contributions of the different cross-streamline vorticity fluxes change (Figure 5-29). For the recirculation as a whole, it is the mean baroclinic flux $\overline{\mathbf{u}}_{\tau} \nabla^{2} \bar{\tau}$ that acts to strengthen the circulation, while all the other terms, including the eddy baroclinic flux $\overline{\mathbf{u}_{\tau}^{\prime} \nabla^{2} \tau^{\prime}}$, act to damp it. Toward the center of the recirculation however, it is rather the eddy baroclinic flux $\overline{\mathbf{u}_{\tau}^{\prime} \nabla^{2} \tau^{\prime}}$ that is the largest positive term, while the contribution from the mean baroclinic flux $\overline{\mathbf{u}}_{\tau} \nabla^{2} \bar{\tau}$ becomes almost negligible. Also, toward the center of the recirculation it is the bottom drag that takes over the role of main damping mechanism, while the contribution from the barotropic eddy flux $\overline{\mathbf{u}_{\phi}^{\prime} \nabla^{2} \phi^{\prime}}$ decreases significantly.

\section{Weak bottom friction case - barotropic vorticity budget}

The same kind of analysis of the time-mean barotropic vorticity balance is carried for the case with weak bottom friction, for which it was determined that the system variability is dominated by barotropic basin modes.

In Figure 5-30, the different contributions to the time-averaged barotropic vorticity budget given by Eq.(5.18), are plotted. Because the bottom friction decay rate is a factor of 100 weaker, the bottom friction contribution to the local balance is extremely small. Also, unlike the strong bottom friction case, the time residue term seems to be larger overall. The main balance in the basin is between convergences and divergences of vorticity flux due to advection $\left(-J_{\phi}\right)$ and lateral dissipation. However, both these terms are extremely small as well. A decomposition of the total Jacobian $-J_{\phi}$, indicates that the actual vorticity balance in the weak bottom friction case is between planetary vorticity advection and eddy flux divergence, where the eddy flux divergence is dominated by the barotropic part, $-\nabla \cdot \overline{\left(\mathbf{u}_{\phi}^{\prime} \nabla^{2} \phi^{\prime}\right)}$ (not shown here). This is essentially the same vorticity balance that was used to illustrate how the basin modes drive a time-mean circulation. It can just be speculated that in this case the barotropic eddy fluxes responsible for driving the circulation are due to the 

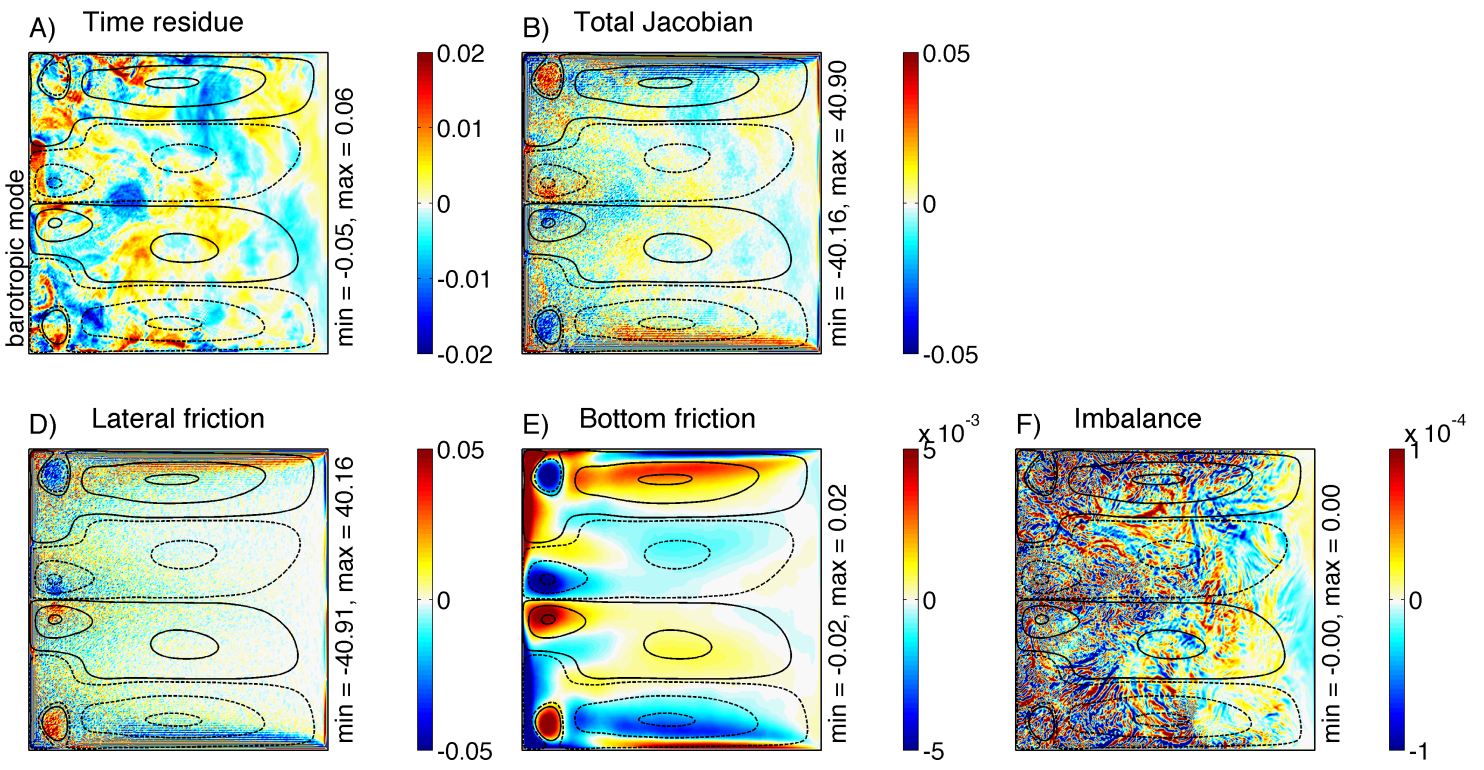

Figure 5-30: Barotropic potential vorticity budget for the case with weak bottom friction $\left(r=10^{-9} s^{-1}\right)$. All terms are as indicated in Eq. (5.18). The missing panel C is the thermal forcing. Selected contours of the time-mean barotropic streamfunction are overlaid in black.
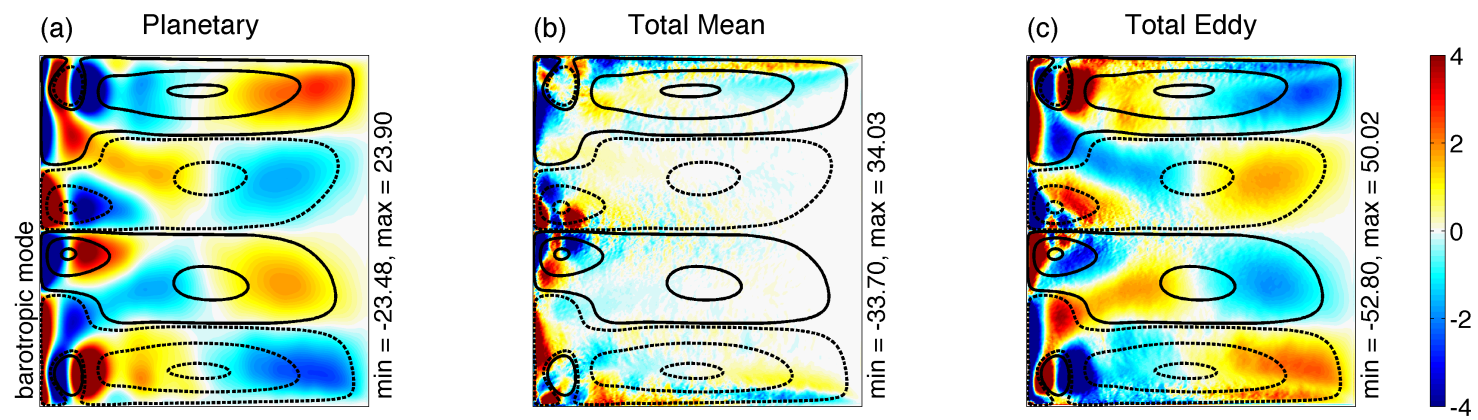

Figure 5-31: Decomposition of the total barotropic Jacobian $-J_{\phi}$, given in panel B) above, into: a), planetary vorticity advection, $-\nabla \cdot(\bar{\phi} \hat{\mathbf{x}})$; b) mean Jacobian or mean vorticity flux convergence, $-\nabla \cdot\left(\overline{\mathbf{u}}_{\phi} \nabla^{2} \bar{\phi}+\delta(1-\delta) \overline{\mathbf{u}}_{\tau} \nabla^{2} \bar{\tau}\right)$; c) eddy Jacobian or eddy vorticity flux convergence, $-\nabla \cdot \overline{\left(\mathbf{u}_{\phi}^{\prime} \nabla^{2} \phi^{\prime}+\delta(1-\delta) \mathbf{u}_{\tau}^{\prime} \nabla^{2} \tau^{\prime}\right)}$.

basin mode variability, and not to smaller scale barotropic variability. Therefore, in the basin interior the dynamics are dominated by the rectification of mean flow by the basin modes.

Again, the integral of the divergence of the different vorticity fluxes is computed for 


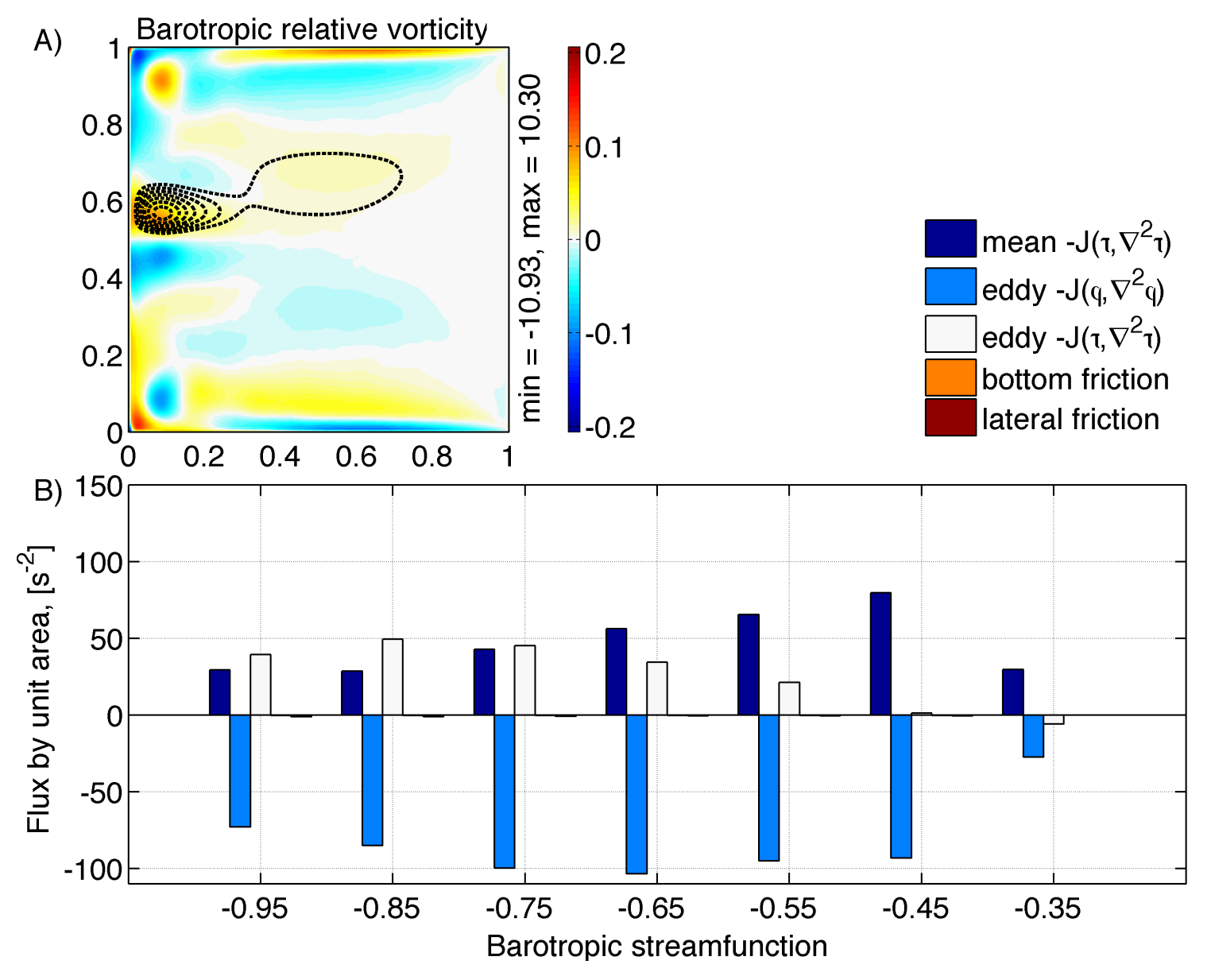

Figure 5-32: Barotropic potential vorticity budget for the case with weak bottom friction $\left(r=10^{-9} s^{-1}\right)$, integrated over a series of regions enclosed by mean barotropic streamlines. Panel A), mean barotropic relative vorticity field scaled with the maximum planetary vorticity. Overlaid are contours of the mean barotropic streamfunction, delimiting the regions of integration, $\frac{\bar{\phi}}{|\min (\bar{\phi})|} \in[-0.35,-0.95]$. Panel B), integral of the vorticity flux divergence by unit area. The different terms are as indicated in Eq. (5.21).

a series of regions enclosed by mean barotropic streamlines, all lying in the northern half-basin. Because in the weak bottom friction case, an interior circulation is driven as well, there are two groups of regions of integration - one laying in the northern recirculation (Figure 5-32), and one situated in the the northern interior gyre (Figure 5-33). Similarly to the strong bottom friction case, the vorticity flux by unit area is plotted in the figures. In Table 5.3 the details of the integrated vorticity balance over 

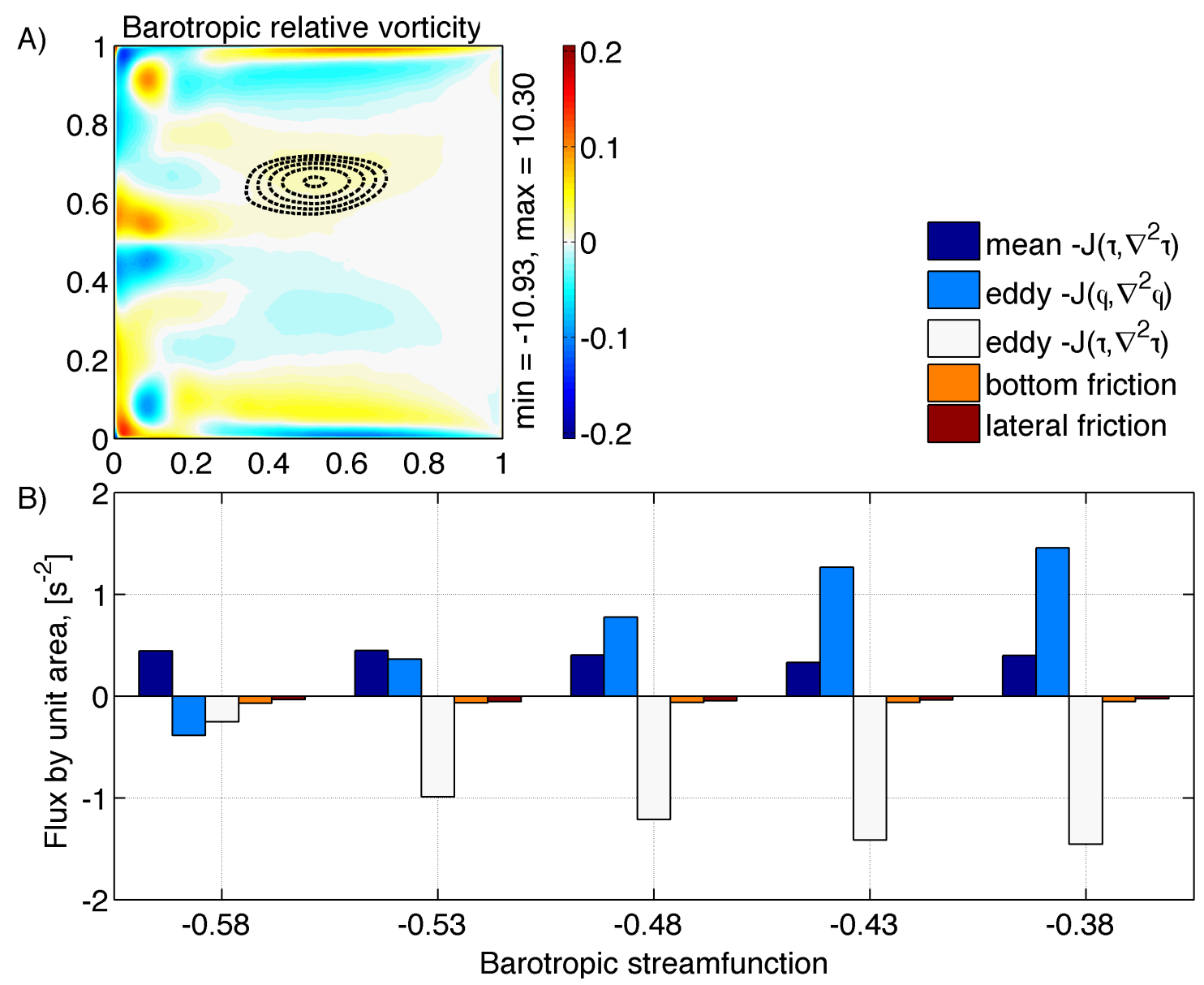

Figure 5-33: Barotropic potential vorticity budget for the case with weak bottom friction $\left(r=10^{-9} s^{-1}\right)$, integrated over a series of regions enclosed by mean barotropic streamlines. Panel A), mean barotropic relative vorticity field scaled with the maximum planetary vorticity. Overlaid are contours of the mean barotropic streamfunction, delimiting the regions of integration, $\frac{\bar{\phi}}{|\min (\bar{\phi})|} \in[-0.38,-0.58]$. Panel B), integral of the vorticity flux divergence by unit area. The different terms are as indicated in Eq. (5.21).

the regions (recirculation and interior), bounded by the streamline where $\bar{\phi}$ is equal to half of its minimum value, are given.

In the recirculation, the cross-streamline vorticity fluxes are similar to what was occurring in the strong bottom friction case. Vorticity is fluxed into the recirculation by the mean baroclinic advection term, $\overline{\mathbf{u}}_{\tau} \nabla^{2} \bar{\tau}$, and the eddy baroclinic advection term, $\overline{\mathbf{u}_{\tau}^{\prime} \nabla^{2} \tau^{\prime}}$ (Figure 5-32). The one big difference is that in the case of weak bottom 
friction, both the lateral diffusion and bottom drag fluxes are very small, close to negligible. Instead, it is the barotropic eddy flux, $\overline{\mathbf{u}_{\phi}^{\prime} \nabla^{2} \phi^{\prime}}$, that takes care of removing vorticity out of the recirculation. Also, unlike for the strong bottom friction case, it is the vorticity flux due to residual time-dependence, that accounts for most of the imbalance (Table 5.3). This suggests that the system is not close to a statistically steady state, even when a time-average over 120 years is used. The likely reason for that is the fact that the system variability is dominated by a number of periodic basin modes. Therefore, a long time-averaging does not necessarily guarantee a statistically steady equilibrium.

The integrated barotropic vorticity budget explains the relationship between bottom drag and recirculation size that we have observed. A priori, one would expect that weak bottom friction favors the recirculations, since less vorticity is exported across the mean streamlines. Thus, the weaker the applied bottom drag, the stronger and larger should be the recirculations. However, we have seen that under a certain threshold decreasing the bottom drag actually leads to smaller and slightly weaker recirculations. The reason for this behavior is that when the bottom friction is decreased, the system variability become dominated by the basin modes. Thus, although the frictional damping becomes very small, the barotropic eddy flux across the mean streamlines increases and over-compensates for it. Overall, the barotropic eddies are so efficient in removing vorticity out of the recirculations, that they actually shrink in size.

What this analysis leaves unanswered is how exactly the barotropic eddy fluxes accomplish that. As already mentioned, the barotropic eddy part of the circulation includes different spatial scales - the Rossby basin mode variability, as well as smaller scale variability. In the basin interior, in all likelihood the barotropic eddy flux is due to the basin mode variability, since it has been demonstrated that the 4-gyre interior flow is driven by the flux resulting for the dominating basin mode. In the region next to the western wall however, the barotropic eddy flux could be due to either the basin modes or smaller scale variability. It is not clear which part acts to increase 
the export of vorticity and consequently to shrink the recirculations.

Concerning the integrated vorticity balance for the interior circulation generated in the weak bottom friction case, it is again the mean baroclinic flux $\overline{\mathbf{u}}_{\tau} \nabla^{2} \bar{\tau}$, that has been scaled to 100 for easier comparison with the other cases in Table 5.3. However,

it can be seen that the barotropic eddy flux $\overline{\mathbf{u}_{\phi}^{\prime} \nabla^{2} \phi^{\prime}}$ contributes almost twice as much in driving the interior gyre. This is expected, given that the interior 4-gyre circulation is rectified by the basin modes. In general, the integrated vorticity budget over the series of interior regions shows that the circulation is driven mainly by the barotropic eddy flux and damped the baroclinic eddy fluxes. Note that the values for the fluxes in the interior gyres are an order of magnitude smaller than in the recirculations.

\subsubsection{Summary of basin modes and recirculations}

To summarize, the goal of the analysis carried in this section was to understand the dynamics of the inertial recirculations, with the more specific question in mind to determine the role played by the basin modes. The following major conclusions were reached:

- By varying the decay rate due to bottom drag, it was established that the basin modes have a strong effect on the inertial recirculations. The weaker the applied bottom friction, the more the system variability is dominated by the basin modes and the smaller and weaker are the recirculations.

- It was shown that in the case of a thermally-only forced ocean, where there is no barotropic vorticity input from external forcing, the recirculations are driven by the mean baroclinic vorticity flux $\overline{\mathbf{u}}_{\tau} \nabla^{2} \bar{\tau}$, and damped out by the barotropic eddy flux $\overline{\mathbf{u}_{\phi}^{\prime} \nabla^{2} \phi^{\prime}}$ and the bottom friction. This is similar to the steady regime, except that in the latter case, since there is no eddy circulation, the bottom drag solely is responsible for damping the recirculations.

- Decreasing the bottom friction in the system increases the variability at all spatial scales, and leads to an increase of the barotropic eddy fluxes $\overline{\mathbf{u}_{\phi}^{\prime} \nabla^{2} \phi^{\prime}}$. 
This is due to a large degree to the fact that the system variability becomes dominated by barotropic basin modes. However, especially near the western wall, it is possible that the basin modes play only an indirect role by leading to an increased barotropic variability on smaller spatial scales.

- In the weak bottom friction limit, the barotropic eddy fluxes are responsible for driving a time-mean 4-gyre circulation in the basin interior. For the recirculations, although the frictional vorticity fluxes become inefficient in transporting vorticity across the mean streamlines, the barotropic eddy fluxes take over that role and are responsible for shrinking the recirculations.

\subsection{Heat budget in the time-dependent regime}

In this section the time-averaged heat budget of the system is examined in order to determine the effect, if any, of the presence of significant basin mode variability.

Because the circulation is time-dependent, there is an additional contribution to the time-averaged heat budget coming from eddy fluxes. More specifically, the timemean vertical velocity $\bar{w}_{i}$ at the interface between the two layers is given by

$$
\begin{aligned}
\bar{w}_{i} & =\overline{\delta_{I}^{2} J(\phi, \eta)}+\bar{w}_{*} \\
& =\delta_{I}^{2} \overline{\mathbf{u}}_{\phi} \cdot \nabla \bar{\eta}+\delta_{I}^{2} \overline{\mathbf{u}_{\phi}^{\prime} \cdot \nabla \eta^{\prime}}+\frac{1}{F \delta_{T}}\left(\bar{\eta}-U_{T} F_{T}(y)\right) .
\end{aligned}
$$

Because the time-averaging is assumed to be over a sufficiently long time period so that a statistically steady equilibrium is reached, the local time rate of change of the interface $\partial \bar{\eta} / \partial t$ is omitted. The time-mean vertical velocity $\bar{w}_{i}$ consists thus of contributions from the mean and eddy advection of interface and the time-mean cross-isopycnal velocity $\bar{w}_{*}$, representing non-adiabatic processes allowing for water properties transformation.

In Figures 5-34 and 5-35, the time-mean vertical velocity and its decomposition into different contributions are plotted for the cases of strong $\left(r=10^{-7} s^{-1}\right)$ and 

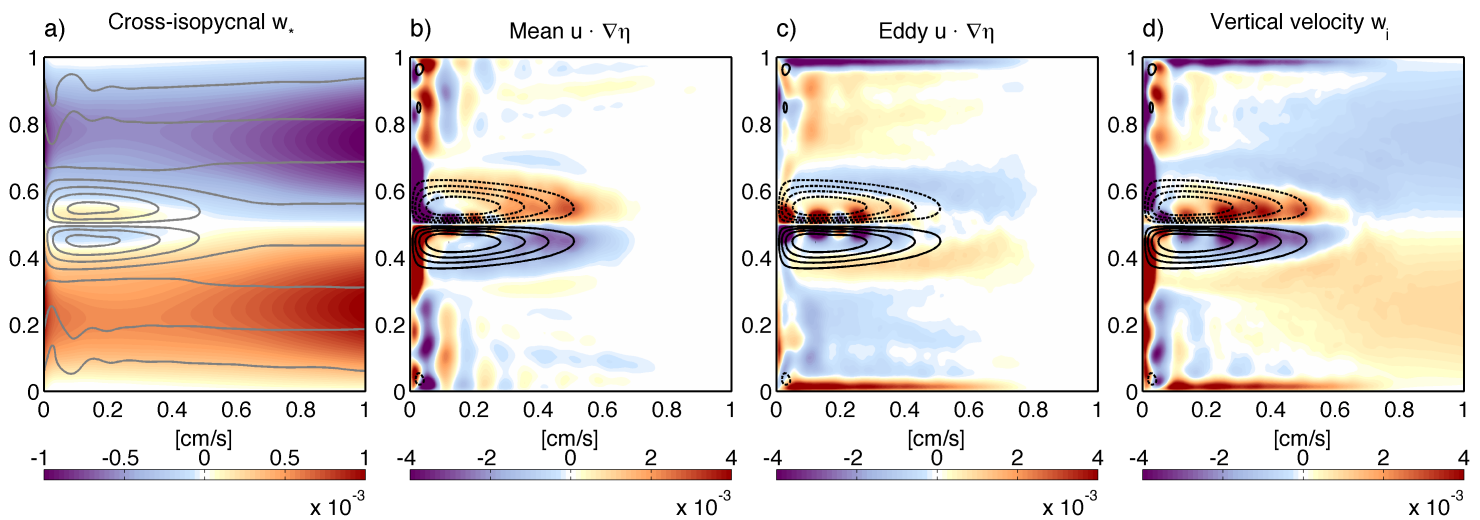

Figure 5-34: Time-mean vertical velocity in the case of a thermally-only forced ocean with strong bottom friction $\left(r=10^{-7} s^{-1}\right)$. Shown are: a) the cross-isopycnal velocity $\left.\bar{w}_{*}=\left(\bar{\eta}-U_{T} F_{T}\right) / F \delta_{T} ; \mathrm{b}\right)$ the mean horizontal advection contribution $\left.\delta_{I}^{2} \overline{\mathbf{u}}_{\phi} \cdot \nabla \bar{\eta} ; \mathrm{c}\right)$ the eddy horizontal advection contribution $\delta_{I}^{2} \overline{\mathbf{u}_{\phi}^{\prime} \cdot \nabla \eta^{\prime}} ;$ d) the net time-mean vertical velocity at the interface $\bar{w}_{i}$. Overlaid in gray on panel a) are select geostrophic contours $\hat{\phi}=y+\Omega \bar{\phi}$, and in black on panels b-d) select mean barotropic streamlines.
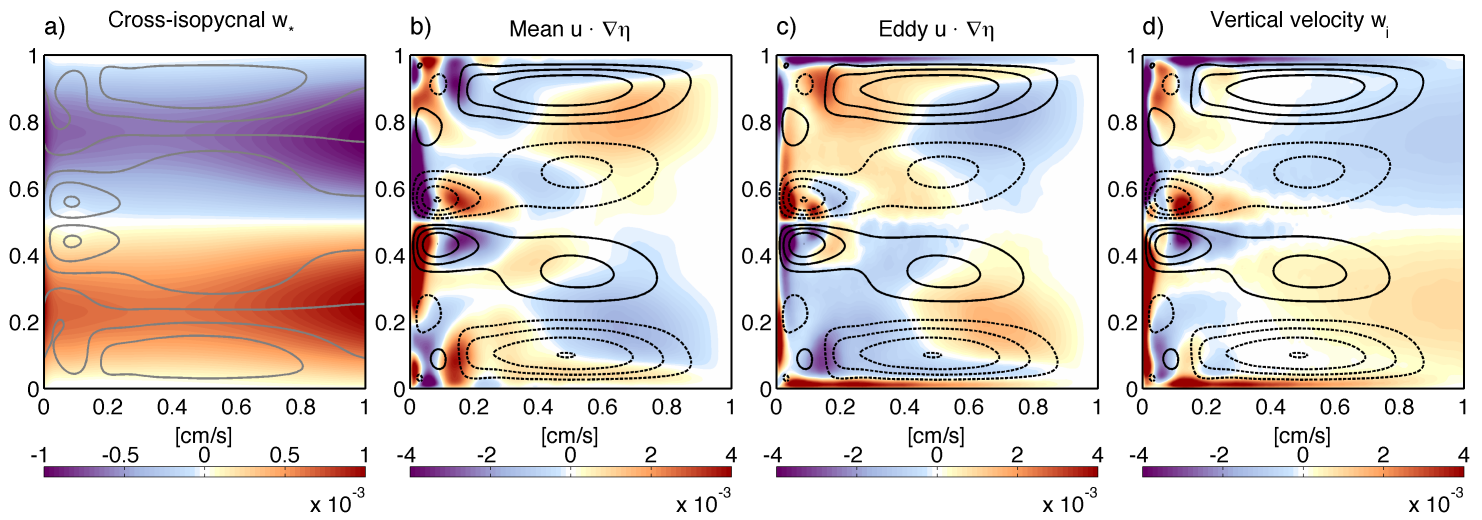

Figure 5-35: Time-mean vertical velocity in the case of a thermally-only forced ocean with weak bottom friction $\left(r=10^{-9} s^{-1}\right)$. Shown are: a) the cross-isopycnal velocity $\left.\bar{w}_{*}=\left(\bar{\eta}-U_{T} F_{T}\right) / F \delta_{T} ; \mathrm{b}\right)$ the mean horizontal advection contribution $\left.\delta_{I}^{2} \overline{\mathbf{u}}_{\phi} \cdot \nabla \bar{\eta} ; \mathrm{c}\right)$ the eddy horizontal advection contribution $\left.\delta_{I}^{2} \overline{\mathbf{u}_{\phi}^{\prime} \cdot \nabla \eta^{\prime}} ; \mathrm{d}\right)$ the net time-mean vertical velocity at the interface $\bar{w}_{i}$. Overlaid in gray on panel a) are select geostrophic contours $\hat{\phi}=y+\Omega \bar{\phi}$, and in black on panels b-d) select mean barotropic streamlines.

weak $\left(r=10^{-9} s^{-1}\right)$ bottom friction. Although in both cases the resulting circulation is time-dependent, only for the weak bottom drag scenario the system variability is dominated by resonating Rossby basin modes.

The time-mean cross-isopycnal velocity for both values of the bottom drag has the 
general pattern of cooling in the northern half-basin and warming in the southern halfbasin. At each latitude, the strongest cooling/heating happens next to the eastern and western walls. In the strong bottom friction case, there is a reversal of the sign of the cross-isopycnal velocity at mid-latitude in the the western side of the basin. There is a slight warming within the northern recirculation gyre, and slight cooling within the southern recirculation gyre, while no such feature is evident in the weak bottom drag scenario. This can be explained by the connection that has been established to the geometry of the geostrophic contours. In the strong bottom drag case, the time-mean barotropic circulation is characterized with intense recirculations with horizontal velocities large enough to arrest the westward propagation of the internal Rossby waves. So, similarly to the steady regime, regions of closed geostrophic contours isolated from the eastern wall form, where the cross-isopycnal velocity is of opposite sign. In the weak bottom drag case, the presence of intense basin modes leads to weaker and smaller recirculations. Although again closed geostrophic contours form, this happens very close to the walls in the western boundary layer, or next to the zonal walls. This may be the reason why, although some closed geostrophic contours are present, there is no sign reversal for the cross-isopycnal velocity in this case.

The part of the vertical velocity associated with the local vertical motion of the interface has both a mean and eddy component. Both are several times larger than the cross-isopycnal velocity and are confined to the regions of strong interface slopes and large flow variability. The mean advection is important mostly in the western boundary layer and at the eastern edge of the recirculations, similarly to the nonlinear steady regime calculations. In the weak bottom friction case, where a 4-gyre vertically integrated circulation is driven by the basin modes, there is also some basin scale structure. The eddy advective contribution is on the same order as the mean, and is also significant mostly in the western boundary layer and in the recirculations. There is a strong eddy advective vertical velocity in the zonal boundary layers next to northern and southern walls. 
Overall, the spatial pattern of the time-mean vertical velocity is dominated by the advective adiabatic contributions from the mean and the eddy advection of interface, with the strongest vertical motion occurring in the western boundary layer. This bears similarity to the vertical velocity in the nonlinear steady calculations, where again the advective terms were taking over the cross-isopycnal velocity.

\subsection{Summary}

The goal of this chapter was to examine the strongly nonlinear time-dependent circulation, when a thermal-only forcing is applied to a 2-layer QG ocean. It was determined that in the weak bottom friction limit the system variability is dominated by barotropic basin modes. Thus, a large part of the analysis carried in this chapter was targeted at determining the effect of the basin modes on the time-mean circulation and its variability, with the results applying to any other systems where resonance of basin modes is excited. The following major conclusions were reached:

(1) In the time-dependent strongly nonlinear regime, when typical half-basin cooling / half-basin warming thermal forcing is applied, a significant time-mean barotropic circulation results, that is on the same order as the directly thermallyforced baroclinic part of the circulation. The spatial structure of the vertically integrated circulation is in the form of recirculations in the western part of the basin, plus in some cases a basin-scale multiple gyre flow. The barotropic recirculations are driven by the mean and eddy flux of baroclinic relative vorticity, while the barotropic eddy flux and bottom drag damp the recirculations.

(3) The time variability of the flow is characterized with the resonance of highfrequency barotropic Rossby basin modes with periods, for the basin size considered here, ranging from several days to up to a month. The basin modes become especially dominant if low bottom drag is used. The most important basin mode for the particular case studied here was identified as a low-order basin mode with a $1 \times 2$ horizontal structure and a period of 13.3 days. 
(4) The degree to which the system variability is dominated by the basin modes has a strong effect on the time-mean circulation and its variability. In particular, the following properties of the circulation were directly related to the presence of intense basin modes:

(a) The basin modes drive a time-mean vertically integrated circulation in the form of multiple gyres. Although a basin mode itself has a zero timemean, because of the nonlinearity of the governing dynamics a non-zero time-mean momentum flux results, and a time-mean circulation is driven.

(b) The basin modes force baroclinic flow variability on short timescales similar to their periods. The variability is due to the advection of the mean interface displacement by the basin modes and is in the form of high-frequency gyre undulations and jet meandering.

(c) The presence of basin modes interferes with the size and strength of the inertial recirculations - the more intense are the basin modes, the weaker and smaller are the recirculations. This is due to the fact that stronger basin modes are associated with larger barotropic eddy fluxes out of the recirculation, and thus more effective damping.

(5) The spatial distribution of the time-mean vertical velocity at the interface between the two density layers is dominated in the time-dependent regime by the mean and eddy components due to the advection of the interface by the barotropic flow. The strongest vertical motions happen thus in the regions of steepest interface slopes and largest flow variability, i.e the boundary layers and the recirculations. 


\section{Chapter 6}

\section{Onset of time-dependence in a thermally-forced ocean}

So far in this thesis we have examined the circulation in a 2-layer thermally-forced QG ocean in both the weakly nonlinear steady regime (Chapter 4), and the strongly nonlinear time-dependent regime (Chapter 5). The results presented in this chapter can be seen as a continuation of the study of the low Reynolds number circulation, with the focus put on examining how the thermally-forced double-gyre flow becomes linearly unstable and time-dependence arises.

It is shown that for some choice of parameters the thermally-forced circulation becomes unstable to perturbations that have a basin-scale spatial structure with timescales and phase propagation characteristics similar to those of barotropic Rossby basin modes. For other choice of parameters, the flow becomes unstable instead to perturbations that are spatially localized and characterized by longer inter-monthly timescales. It is argued that the difference in the destabilizing perturbations is due to the difference in the geometry of the geostrophic contours. Comparisons are drawn to the wind-driven double-gyre flow, where similar dependence can be uncovered. 


\subsection{Introduction}

There is a very large literature on the problem of the internal variability of the winddriven circulation. By internal variability we mean the variability that arises under steady forcing conditions because of the nonlinear dynamics. Understanding the internal modes of variability of the ocean circulation is important because of the role they may play in setting the low-frequency ocean variability and its effect on climate.

One little piece of this problem is to determine how temporal variability arises in a model of the ocean circulation when the Reynolds number is increased. Of particular interest for us is the study by Dijkstra and Katsman (1997), where the initial bifurcations of the wind-driven double-gyre flow in a 2-layer QG model are examined. They show that when the lateral viscosity of the model is decreased below a critical threshold, the circulation transitions from steady to oscillatory behavior due to baroclinic instabilities. The temporal variability consists of meandering and local and temporal weakening and strengthening of the midlatitude jet at inter-monthly timescales. We will use the results by Dijkstra and Katsman (1997) as a base for comparison when examining the stability of the thermally-forced double-gyre circulation.

Determining the stationary solutions and first few perturbations to which a circulation becomes unstable is an interesting problem in itself, but it can also provide clues for the system behavior in the strongly nonlinear regime. Although the stable equilibria found at low Reynolds numbers may become unstable at larger Reynolds numbers, they may still be able to influence the system time-dependent behavior (Primeau, 1998).

Our goal in this chapter is to compute the linear stability of a thermally-forced 2-layer QG ocean, a problem not studied until now, in order to determine how timedependence appears. We are interested in finding the temporal and spatial scales of the variability, as well as the instability processes responsible for it. It is possible that some of the results may shed light on the reason why the variability of the thermallyforced circulation in the strongly nonlinear regime, that we studied in Chapter 5, was 
dominated by barotropic Rossby basin modes.

The presentation of this chapter is organized as follows. In Section 6.2 the stability problem is formulated and some tools for analyzing and understanding the solutions are presented. In Section 6.3 we examine the stability properties and onset of time-dependence in a thermally-forced ocean, while in Section 6.4 the stability of an equivalent wind-forced configuration is considered. Some final conclusions and remarks are given in Section 6.5.

\subsection{Problem formulation and approach}

\subsubsection{Nondimensional parameters and governing equations}

When describing the wind- and thermally-forced QG model in Chapter 3, we determined that the behavior of the flow is controlled by a set of 7 nondimensional parameters, once a velocity scale $U$ set by either the wind stress or the thermal forcing is chosen. For all stability calculations performed in this chapter we have reduced the number of nondimensional parameters to 6 , by eliminating the bottom friction, since this parameter has been judged nonessential when determining the stability of a circulation closed through a Munk boundary layer. If the circulation was closed instead through a Stommel boundary layer or if values for $\delta_{S}$ were considered such that the equilibrium state of the system is affected (Primeau, 1998; Simonnet, 2005), then one can not exclude the bottom friction from the stability analysis. However, all calculations presented in this thesis were limited to the regime $\delta_{S} \ll \delta_{M}$, with the bottom friction used exclusively as a way to damp the barotropic basin modes and control the degree to which they dominate the system variability. Its role in setting the steady regime solutions was shown to be nearly negligible, and thus we have de-

cided to all together eliminate the bottom friction from the model when performing stability analyses.

For all stability calculations in this chapter we have used a slightly different nondimensionalization of the QG equations. This does not introduce any new physics to 
the problem, but has the advantage of making the comparisons with previous results about the stability of wind-driven flows more straightforward. The nondimensional QG potential vorticity equations by density layers when an advective timescale $T=L / U$ is used for the nondimensionalization, instead of the barotropic frequency $T=1 / \beta_{0} L$, are written below

$$
\begin{aligned}
\frac{\partial}{\partial t}\left(\nabla^{2} \psi_{1}-F_{1}\left(\psi_{1}-\psi_{2}\right)\right) & +J\left(\psi_{1}, \nabla^{2} \psi_{1}-F_{1}\left(\psi_{1}-\psi_{2}\right)\right)+\beta \frac{\partial \psi_{1}}{\partial x} \\
& =U_{w} F_{w}(y)-F_{1} w_{*}+\frac{1}{R e} \nabla^{4} \psi_{1} \\
\frac{\partial}{\partial t}\left(\nabla^{2} \psi_{2}+F_{2}\left(\psi_{1}-\psi_{2}\right)\right) & +J\left(\psi_{2}, \nabla^{2} \psi_{2}+F_{2}\left(\psi_{1}-\psi_{2}\right)\right)+\beta \frac{\partial \psi_{2}}{\partial x} \\
& =F_{2} w_{*}+\frac{1}{R e} \nabla^{4} \psi_{2} \\
w_{*} & =-\frac{1}{\Omega \delta_{T}}\left(\psi_{1}-\psi_{2}+U_{T} F_{T}(y)\right)
\end{aligned}
$$

The following set of nondimensional parameters appear in the equations

$$
\begin{gathered}
\delta_{12}=\frac{H_{1}}{H_{2}}, \quad \Omega \equiv \frac{F}{\beta}=\frac{U}{\beta_{0} R_{d}^{2}}, \quad \beta=\frac{\beta_{0} L^{2}}{U}, \quad R e=\frac{U L}{\nu}, \quad \delta_{T}=\frac{\gamma \beta_{0} R_{d}^{2}}{L}, \\
U_{T}=\frac{g^{\prime} h_{0}}{U f_{0} L}, \quad U_{w}=\frac{\tau_{0} L}{U^{2} \rho_{0} H_{1}} .
\end{gathered}
$$

where the notations $F_{1}=\left(H_{2} / H\right) F=\beta \Omega /\left(1+\delta_{12}\right)$ and $F_{2}=\left(H_{1} / H\right) F=\delta_{12} F_{1}$ have been used for shorter writing.

This is an equivalent group of nondimensional parameters to the ones described in Chapter 3 that can be obtained by simple rearrangement*. The ratio $\delta_{12}$ of the upper to the lower layer depth is used instead of the ratio $\delta=H_{1} / H$, and the Reynolds number instead of the Munk boundary layer $\delta_{M}$ as a measure of the importance of lateral friction. Also, two new nondimensional parameters are introduced. The

* For reference, the nondimensional parameters as derived in Chapter 3 when the barotropic frequency $1 / \beta_{0} L$ is used for a timescale are reproduced below

$$
\delta=\frac{H_{1}}{H}, F=\frac{L^{2}}{R_{d}^{2}}, \delta_{I}=\frac{U}{\beta_{0} L^{2}}, \delta_{M}=\frac{\nu}{\beta_{0} L^{3}}, \delta_{T}=\frac{\gamma \beta_{0} R_{d}^{2}}{L}, U_{T}=\frac{g^{\prime} h_{0}}{U f_{0} L}, U_{w}=\frac{\tau_{0}}{U \rho_{0} \beta_{0} H_{1} L} .
$$


nondimensional $\beta$ parameter is simply equal to the inverse of the square inertial boundary layer, and thus similarly to $\delta_{I}$ measures the relative importance of the nonlinear advection of relative vorticity to the planetary vorticity advection. The parameter $\Omega$ is equal to the ratio of the flow speed to that of a long internal Rossby wave and has been brought in instead of the Froude number $F=L^{2} / R_{d}^{2}$. We have seen previously this parameter in the definition of the geostrophic contours $\hat{\phi}=y+\Omega \phi$.

There is an extra 7th parameter present in the list of nondimensional parameters because of the undetermined velocity scale $U$. For the case of a wind-driven ocean, the velocity scale is usually chosen assuming a linear Sverdrup vorticity balance, which implies $U_{w}=\beta$ and a velocity scale set by the wind stress, $U=\tau_{0} / \beta_{0} \rho_{0} H_{1} L$. In the wind-only forced case, the circulation is controlled thus by a set of 4 parameters: $\delta_{12}, \Omega, \beta$ and Re. In the more general case of a wind- and thermally-forced ocean, there are two additional parameters, $\delta_{T}$ and $U_{T}$, that describe the thermal relaxation forcing. If the same wind-derived velocity scale $U$ is kept, then the parameter $U_{T}$ represents the ratio of the thermally-driven to the wind-driven baroclinic horizontal velocity, as determined from a linear vorticity balance (see discussion in Chapter 3). Finally, in the special case of a thermally-only forced ocean, $U_{w}=0$ and the velocity scale is set rather by the thermal forcing. In this case the circulation is controlled by a set of 5 parameters: $\delta_{12}, \Omega, \beta, \delta_{T}$ and $R e$. However, even in this case it is useful to define an "equivalent" wind stress, in the sense that it generates a circulation with the same speed $U$ as the one driven by the thermal forcing alone. This provide us with a way to judge the strength of the applied thermal forcing, as well as to compare wind-driven and thermally-driven flows.

For all stability calculations presented in this chapter we will use the Reynolds number as a control parameter. This signifies that for all other parameters held fixed $\left(\delta_{12}, \Omega, \beta, \delta_{T}\right.$ and $U_{T}$ specified) we will vary the Reynolds number until the threshold for linear stability of the circulation is determined. In addition, we will explore the dependence on the $\Omega$ parameter of the properties of the destabilizing perturbations in terms of their spatial structure, time periods and energy budgets. 


\subsubsection{Stability analysis of an equilibrium solution}

Suppose that $\Psi_{1}(x, y)$ and $\Psi_{2}(x, y)$ are the streamfunctions by density layers for an equilibrium solution (or stationary solution) of the governing equations (6.1)-(6.2), and let $Q_{1}$ and $Q_{2}$ designate the potential vorticities associated with this solution

$$
Q_{1}=\beta y+\nabla^{2} \Psi_{1}-F_{1}\left(\Psi_{1}-\Psi_{2}\right), \quad Q_{2}=\beta y+\nabla^{2} \Psi_{2}+F_{2}\left(\Psi_{1}-\Psi_{2}\right) .
$$

The stability of the equilibrium state $\left\{\Psi_{1}, \Psi_{2}\right\}$ can be determined if a perturbation $\left\{\psi_{1}^{\prime}, \psi_{2}^{\prime}\right\}$ is added to the solution and its time-evolution examined, where $\left\{\psi_{1}^{\prime}, \psi_{2}^{\prime}\right\}$ is of the form

$$
\psi_{n}^{\prime}(x, y, t)=\operatorname{Real}\left(\hat{\psi}_{n}(x, y) e^{\sigma t}\right)=\operatorname{Real}\left(\hat{\psi}_{n}(x, y) e^{\left(\sigma_{r}+i \sigma_{i}\right) t}\right), \quad n=1,2 .
$$

The complex amplitudes $\hat{\psi}_{n}(x, y)$ describe the spatial structure of the perturbation flow, while the growth rate $\sigma$, its temporal structure. The real part of the growth rate $\sigma$ expresses whether the perturbation grows or decays in time and is indicative therefore for whether the equilibrium solution $\left\{\Psi_{1}, \Psi_{2}\right\}$ is stable or unstable to the perturbation $\left\{\psi_{1}^{\prime}, \psi_{2}^{\prime}\right\}$. The imaginary part of the growth rate $\sigma$ distinguishes between oscillating perturbations with $\sigma_{i} \neq 0$ and stationary perturbations with $\sigma_{i}=0$. If a flow becomes unstable to an oscillating perturbation, then temporal variability with period $T=2 \pi / \sigma_{i}$ arises. Instability to a stationary perturbation on the other hand leads to multiple equilibrium solutions but no temporal variability.

For as long as the amplitude of the perturbation remains small, i.e. for times $t \ll 1 /\left|\sigma_{r}\right|$, its evolution is described by the perturbation QG potential vorticity equations, linearized around the equilibrium state $\left\{\Psi_{1}, \Psi_{2}\right\}$, 


$$
\begin{aligned}
\left(\sigma+\frac{\partial \Psi_{1}}{\partial x} \frac{\partial}{\partial y}-\frac{\partial \Psi_{1}}{\partial y} \frac{\partial}{\partial x}\right)\left(\nabla^{2} \hat{\psi}_{1}-F_{1}\left(\hat{\psi}_{1}-\hat{\psi}_{2}\right)\right) & +J\left(\hat{\psi}_{1}, Q_{1}\right) \\
& =d_{2} \frac{\beta}{\delta_{T}}\left(\hat{\psi}_{1}-\hat{\psi}_{2}\right)+\frac{1}{R e} \nabla^{4} \hat{\psi}_{1} \\
\left(\sigma+\frac{\partial \Psi_{2}}{\partial x} \frac{\partial}{\partial y}-\frac{\partial \Psi_{2}}{\partial y} \frac{\partial}{\partial x}\right)\left(\nabla^{2} \hat{\psi}_{2}+F_{2}\left(\hat{\psi}_{1}-\hat{\psi}_{2}\right)\right) & +J\left(\hat{\psi}_{2}, Q_{2}\right) \\
& =-d_{1} \frac{\beta}{\delta_{T}}\left(\hat{\psi}_{1}-\hat{\psi}_{2}\right)+\frac{1}{R e} \nabla^{4} \hat{\psi}_{2}
\end{aligned}
$$

with the boundary conditions $\hat{\psi}_{n}=\nabla \hat{\psi}_{n} \cdot \hat{\mathbf{n}}=0$ applied on all walls. In the equations above, the notation $d_{n}=H_{n} / H$ is used.

The perturbation equations reveal a slight difference between the wind and the thermal forcing - while part of the thermal relaxation forcing consisting of restoring of the perturbation interface to zero is present, the wind stress is absent all together. In general, a characteristic of the perturbation equations is that the external forces that drive the equilibrium solution do not appear in them. They influence the perturbation evolution only indirectly, by setting the background potential vorticity gradients and providing the energy on which the perturbations could eventually grow. The thermal relaxation forcing is special in the sense that it is determined from internal dynamics, and not prescribed externally like the wind stress. It influences thus the perturbation dynamics. Nonetheless, it acts only as an additional damping and does not drive the perturbation flow.

From a mathematical point of view, equations (6.7) and (6.8) when discretized form a generalized eigenvalue problem of the form $A \hat{\psi}=\sigma B \hat{\psi}$. The possible different perturbation amplitudes $\hat{\psi}=\left\{\hat{\psi}_{1}, \hat{\psi}_{2}\right\}$ are given by the set of eigenvectors of this problem, while their growth rates $\sigma$, by the corresponding eigenvalues. When solving the eigenvalue problem we are interested in finding only the most unstable eigenmodes, i.e the ones with the largest real growth rates. The threshold of linear stability of the circulation to a given perturbation is given by the value of the control parameter (the Reynolds number) for which its eigenvalue crosses the real axis. In 
the calculations presented next, our goal is to determine the first few perturbations to which the flow becomes unstable and to examine their spatial and temporal structure.

Note that because of the symmetry properties of the QG equations, the solutions of the perturbations equations (6.7) and (6.8) come in pairs. If $\left\{\hat{\psi}_{1}, \hat{\psi}_{2}\right\}$ is a solution for a growth rate $\sigma=\sigma_{r}+i \sigma_{i}$, then $\left\{\hat{\psi}_{2}^{*}, \hat{\psi}_{1}^{*}\right\}$ is a solution as well, but for a growth rate $\sigma^{*}=\sigma_{r}-i \sigma_{i}$, where the star denotes complex conjugation. Thus, the oscillatory perturbation solutions come in pairs leading to an eigenvalue spectrum that is symmetric with respect to the imaginary axis. When examining the oscillatory perturbations it is enough therefore to find only one of them.

\subsubsection{Perturbation energy equations}

A useful tool for analyzing the destabilizing perturbations that can give us an insight into their origin is the perturbation energy budget. The total perturbation energy integrated over the whole basin is given in terms of the complex amplitudes $\hat{\psi}_{n}$ by (Pedlosky, 1987)

$$
E=\frac{1}{2} \iint d_{1}\left|\nabla \hat{\psi}_{1}\right|^{2}+d_{2}\left|\nabla \hat{\psi}_{2}\right|^{2}+d_{1} d_{2} F\left|\hat{\psi}_{1}-\hat{\psi}_{2}\right|^{2}
$$

The perturbation energy is equal to the sum of the kinetic energy by density layers and the potential energy associated with the interface displacement for the perturbation flow.

An equation for the evolution of the perturbation energy averaged over one oscillation period can be obtained if the perturbation equations (6.7) and (6.8) are multiplied by respectively $d_{1} \hat{\psi}_{1}^{*}$ and $d_{2} \hat{\psi}_{2}^{*}$, added together and integrated over the area of the basin. The final result after performing these operations is given below, with a more detailed derivation provided in Appendix D, 


$$
\begin{aligned}
\sigma_{r} E & =\underbrace{\iint d_{1} \hat{\psi}_{1}^{*} J\left(\Psi_{1}, \nabla^{2} \hat{\psi}_{1}\right)}_{B T_{1}}+\underbrace{\iint d_{2} \hat{\psi}_{2}^{*} J\left(\Psi_{2}, \nabla^{2} \hat{\psi}_{2}\right)}_{B T_{2}} \\
& +\underbrace{\iint d_{1} d_{2} F\left[\hat{\psi}_{1}^{*} J\left(\Psi_{1}, \hat{\psi}_{2}-\hat{\psi}_{1}\right)+\hat{\psi}_{2}^{*} J\left(\Psi_{2}, \hat{\psi}_{1}-\hat{\psi}_{2}\right)\right]}_{\text {BC }} \\
& +\iint d_{1} \hat{\psi}_{1}^{*} J\left(\hat{\psi}_{1}, Q_{1}\right)+d_{2} \hat{\psi}_{2}^{*} J\left(\hat{\psi}_{2}, Q_{2}\right) \\
& -\underbrace{\iint d_{1} d_{2} \frac{\beta}{\delta_{T}}\left|\hat{\psi}_{1}-\hat{\psi}_{2}\right|^{2}+\frac{d_{1}}{R e} \hat{\psi}_{1}^{*} \nabla^{4} \hat{\psi}_{1}+\frac{d_{2}}{R e} \hat{\psi}_{2}^{*} \nabla^{4} \hat{\psi}_{2}}_{\text {dissipation }} .
\end{aligned}
$$

When writing this equation, it is understood that the real part of the right-hand side is taken.

For an unstable perturbation, the growth rate $\sigma_{r}$ and thus the rate of change of the perturbation energy $\sigma_{r} E$ over an oscillation period are positive, with the different sources and sinks sustaining the growth listed in the right-hand side of the equation. The first three integrals, denoted by $B T_{n}$ and $B C$, are associated with the advection by the equilibrium flow $\Psi_{n}$ of the perturbation relative and stretching vorticities for the two density layers. They represent the possible sources of perturbation energy and correspond respectively to barotropic and baroclinic type of instability of the flow. The next integral involving the advection by the perturbation flow of the equilibrium potential vorticity $Q_{n}$ vanishes because of the no-normal flow boundary conditions and thus does not contribute to the perturbation energy budget. Finally, there is also a sink of perturbation energy due to the lateral diffusion of relative vorticity and eventually, in the case of a thermally-forced ocean, due to the relaxation of the interface.

By applying the divergence theorem and using the boundary conditions it can be shown that the source terms $B T_{n}$ and $B C$ can be rewritten equivalently as 


$$
\begin{aligned}
B T_{n} & =\iint d_{n} \hat{\psi}_{n}^{*} J\left(\Psi_{n}, \nabla^{2} \hat{\psi}_{n}\right) \\
& =\iint d_{n} \frac{\partial \hat{\psi}_{n}^{*}}{\partial x_{i}} \frac{\partial \hat{\psi}_{n}}{\partial x_{j}} \frac{\partial U_{n, j}}{\partial x_{i}} \\
& =\iint d_{n}\left[\left|\frac{\partial \hat{\psi}_{n}}{\partial x}\right|^{2} \frac{\partial U_{n}}{\partial x}+\frac{\partial \hat{\psi}_{n}^{*}}{\partial x} \frac{\partial \hat{\psi}_{n}}{\partial y}\left(\frac{\partial V_{n}}{\partial x}+\frac{\partial U_{n}}{\partial y}\right)+\left|\frac{\partial \hat{\psi}_{n}}{\partial y}\right|^{2} \frac{\partial V_{n}}{\partial y}\right] \\
B C & =\iint d_{1} d_{2} F\left[\hat{\psi}_{1}^{*} J\left(\Psi_{1}, \hat{\psi}_{2}-\hat{\psi}_{1}\right)+\hat{\psi}_{2}^{*} J\left(\Psi_{2}, \hat{\psi}_{1}-\hat{\psi}_{2}\right)\right] \\
& =\iint d_{1} d_{2} F \hat{\psi}_{1}^{*} J\left(\Psi_{1}-\Psi_{2}, \hat{\psi}_{2}\right) \\
& =\iint d_{1} d_{2} F\left[\hat{\psi}_{1}^{*} \frac{\partial \hat{\psi}_{2}}{\partial x}\left(U_{1}-U_{2}\right)+\hat{\psi}_{1}^{*} \frac{\partial \hat{\psi}_{2}}{\partial y}\left(V_{1}-V_{2}\right)\right]
\end{aligned}
$$

where $U_{n}=-\partial \Psi_{n} / \partial y$ and $V_{n}=\partial \Psi_{n} / \partial x$ represent the equilibrium state velocities.

The barotropic energy conversion term is proportional thus to the horizontal shear of the equilibrium velocity while the baroclinic conversion term, to the vertical shear. Determining which one of these conversion terms is positive for a specific perturbation, allows us to qualify the instability mechanism responsible for the growth as either barotropic, baroclinic or mixed barotropic-baroclinic.

\subsubsection{Numerical methods}

For all calculations presented in this chapter the QG equations are solved using a continuation code as in Dijkstra and Katsman (1997). This means that the equilibrium solutions are determined not through time-stepping but directly using a Newton type solver and an arc-length continuation algorithm that allows stationary solutions to be followed when a control parameter is varied. The stability of the equilibrium solution is then computed by solving the generalized eigenvalue problem defined by equations (6.7) and (6.8). The details of the numerical methods used in the continuation code can be found in Dijkstra et al. (1995).

The equations are solved on a non-equidistant grid with size $65 \times 65$, as shown in 

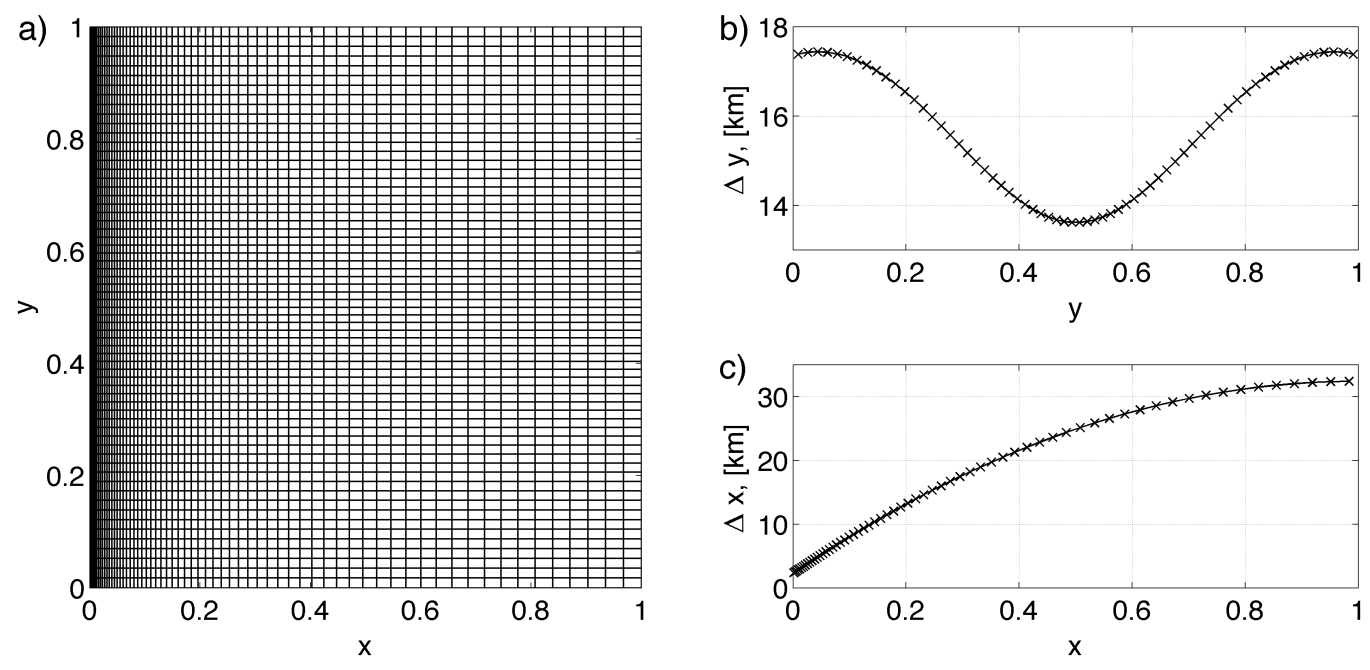

Figure 6-1: a): Stretched grid used in the continuation code. b) and c): Grid resolution in the meridional and the zonal directions for a square basin of size $L=1000 \mathrm{~km}$.

Figure 6-1. The stretched grid is advantageous since it provides finer resolution in the parts of the basin where strong solution gradients are expected, while simultaneously it limits the size of the discretized problem. For a square basin of width $L=1000 \mathrm{~km}$ the zonal resolution varies from $2.4 \mathrm{~km}$ in the western boundary layer to $32.4 \mathrm{~km}$ in the eastern part of the basin. The meridional resolution is $13.6 \mathrm{~km}$ at midlatitude and increases to $17.4 \mathrm{~km}$ next to the southern and northern walls.

For all calculations, the variation of the forcing term with latitude, be it thermal $F_{T}(y)$ or wind $F_{w}(y)$, is described by a sine function, $F_{T}(y)=F_{w}(y)=-\sin (2 \pi y)$.

\subsection{Stability of a thermally-forced double-gyre flow}

One of the main goals of this chapter is to examine the onset of time-dependence in a thermally-forced ocean. In order to do that we have chosen a model configuration very similar to the one used in Chapter 4, where the steady thermally-forced circulation was analyzed. The values of all dimensional and nondimensional parameters used in the stability calculations are listed in Table 6.1. The one difference from the Chapter 4 configuration is that although an ocean of the same total depth $H=3000 \mathrm{~m}$ is 
considered, the upper layer is taken slightly deeper, $H_{1}=860 \mathrm{~m}$ instead of $H_{1}=$ $500 \mathrm{~m}$. This is done in order to have a value for the ratio $\delta_{12}=H_{1} / H_{2}$ that matches the one used in Dijkstra and Katsman (1997) for comparison with their results.

We have performed calculations for two different values of the $\Omega$ parameter or equivalently the Froude number $F$. Varying the $\Omega$ parameter can be thought as varying the stratification of the ocean while keeping all other nondimensional parameters the same. Decreasing the stratification leads to smaller internal deformation radius and slower propagating long Rossby waves. This translates into a larger value for the $\Omega$ parameter, which is equal to the ratio of the flow speed to that of the long internal Rossby waves, $\Omega=U / \beta_{0} R_{d}^{2}$.

In Figure 6-2 the barotropic circulation overlaid with the geostrophic contours $\hat{\phi}=y+\Omega \phi$ is plotted for the two values $\Omega=1.2$ and $\Omega=0.3$. In both cases, the same low Reynolds number $R e=12$ is used resulting into a viscous, steady circulation. As expected for a thermally-forced ocean, the magnitude of the barotropic flow is very weak with its spatial pattern nearly identical for both values of the $\Omega$ parameter. What changes between the two calculations more significantly is the geometry of the geostrophic contours. For $\Omega=1.2$ the Rossby waves are slow enough, so that even the weak barotropic flow generated in a thermally-forced ocean manages to disturb their propagation, as evident from the distorted geostrophic contours in the western side of the basin. For $\Omega=0.3$ the Rossby waves are too fast and the geostrophic contours are nearly latitude lines.

Although the circulation in the low Reynolds limit looks similar for both values of $\Omega$, its stability properties are quite different. In order to determine the threshold for instability we have followed the equilibrium solution for each value of $\Omega$ as the Reynolds number is increased. In Figure 6-3 the maximum of the baroclinic streamfunction, which is used as a norm of the solution, is plotted as a function of the Reynolds number. Because in a thermally-forced ocean both layers are in motion, the advection of stretching vorticity and thus the value of the $\Omega$ parameter matters for determining the equilibrium solution. It can be seen that for a given Reynolds 


\begin{tabular}{c|ccc|cc}
\hline \hline & $L$ & $1000 \mathrm{~km}$ & $f_{0}$ & $1 \times 10^{-4} \mathrm{~s}^{-1}$ \\
Dimensional & $H_{1}$ & $860 \mathrm{~m}$ & $\beta_{0}$ & $2 \times 10^{-11} \mathrm{~m}^{-1} \mathrm{~s}^{-1}$ \\
parameters & $H_{2}$ & $2140 \mathrm{~m}$ & $U$ & $0.02 \mathrm{~ms}^{-1}$ \\
& $\rho_{0}$ & $1000 \mathrm{kgm}^{-3}$ & & & \\
\hline Nondimensional & $\delta_{12}=$ & 0.4 & $U_{T}=$ & 2 \\
parameters & $\beta$ & 1000 & $\delta_{T}=$ & \\
& $\left(U_{w}=\right.$ & $0)$ & & \\
\hline \hline
\end{tabular}

Table 6.1: Dimensional and nondimensional parameters used for the thermally-forced stability calculations. Two different values of the internal deformation radius are applied, leading to two different values for the $\Omega$ parameter, or alternatively the Froude number $F$. The Reynolds number is not specified, since it is used as a control parameter.

A)

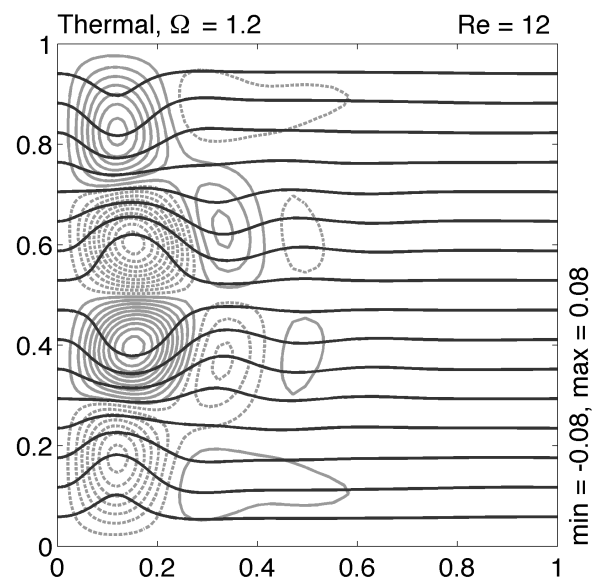

B)

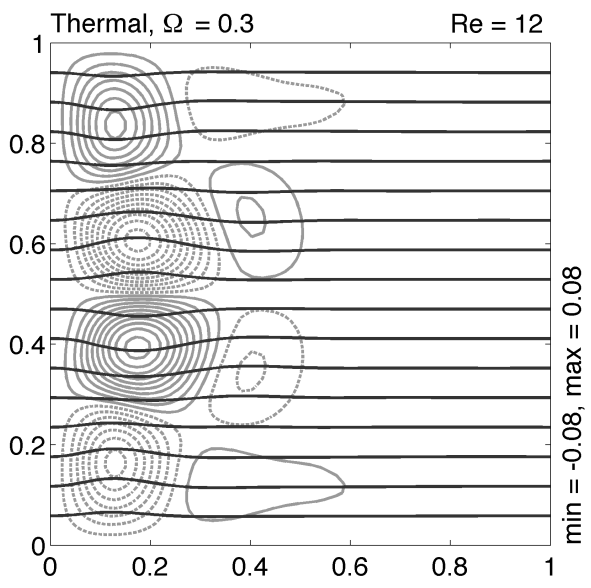

Figure 6-2: Barotropic circulation in gray and geostrophic contours $\hat{\phi}=y+\Omega \phi$ in black for a thermally-forced ocean with A) $\Omega=1.2\left(R_{d}=28.8 \mathrm{~km}\right)$, and B) $\Omega=0.3$ $\left(R_{d}=57.7 \mathrm{~km}\right)$. In both cases, $R e=12$ is used and the resulting circulation is steady.

number, the larger the value of $\Omega$, the smaller is the maximum of the baroclinic streamfunction. Only in the very low Reynolds number limit, when the nonlinear advective terms are negligible, is the circulation independent of $\Omega$.

The threshold for instability of the circulation is determined by the point $H_{1}$ where the first pair of eigenvalues crosses the real axis (Figure 6-3). This happens 


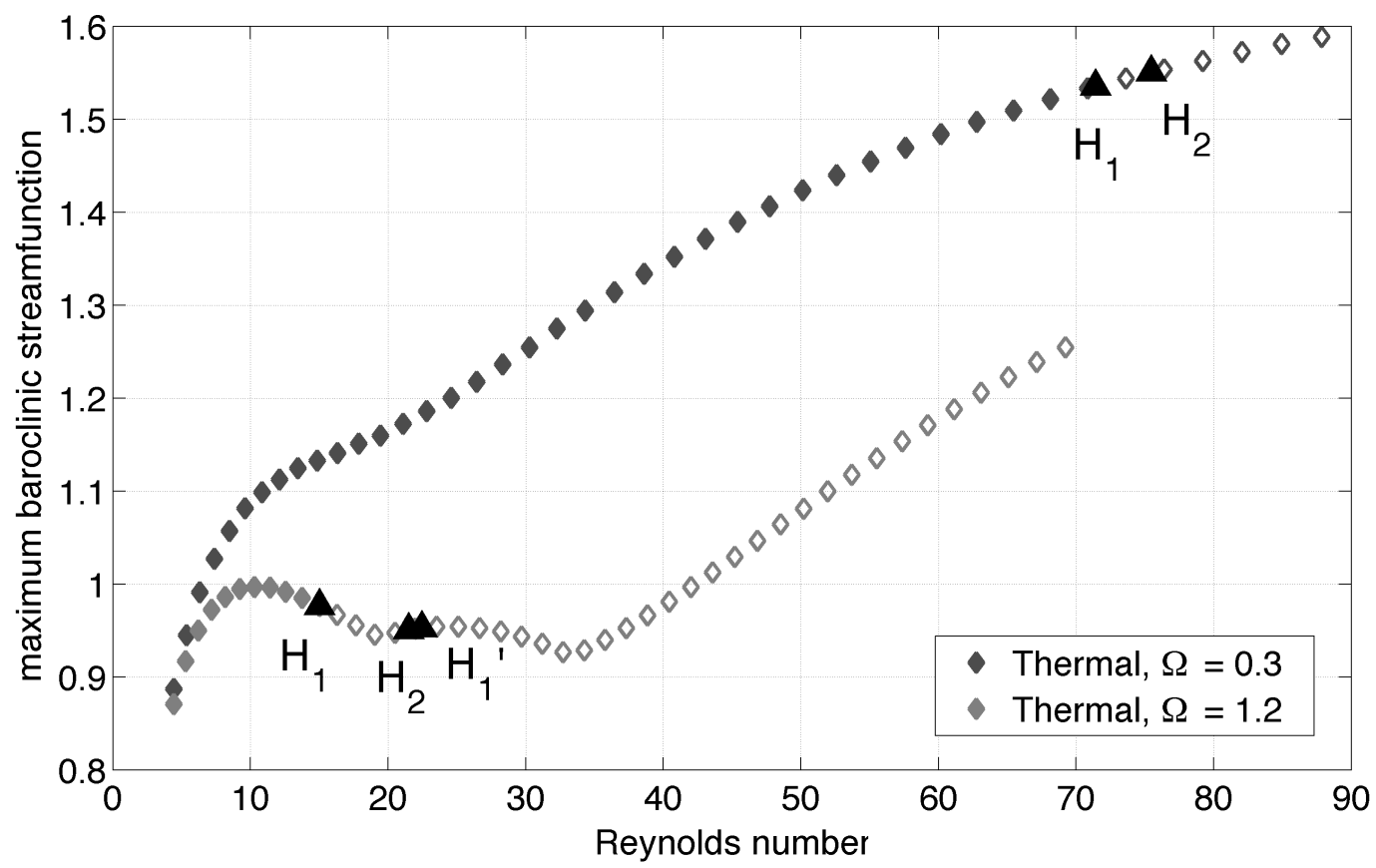

Figure 6-3: Maximum of the baroclinic streamfunction as a function of the Reynolds number for $\Omega=1.2$ and $\Omega=0.3$ in the case of a thermally-forced ocean. Filled symbols indicate stable equilibrium states, for which all eigenvalues have negative real parts. Empty symbols indicate unstable equilibrium states, for which there is at least one eigenvalue with positive real part. The approximative Reynolds numbers at which an eigenvalue crosses the real axis are shown in triangles and denoted by $H_{n}$, which stands for an instability to an oscillating perturbation (Hopf bifurcation).

at approximatively $R e_{H_{1}}=15$ for the case with $\Omega=1.2\left(\right.$ or $R_{d}=28.8 \mathrm{~km}$ ) and at $R e_{H_{1}}=73$ for the case with $\Omega=0.3$ (or $R_{d}=57.7 \mathrm{~km}$ ). Thus, not surprisingly, the strongly stratified ocean is more stable. In Figure 6-4 the equilibrium solution at the critical Reynolds number $R e_{H_{1}}$, where the flow becomes unstable, is plotted by vertical modes. The magnitude of both the barotropic and baroclinic streamfunction are larger for $\Omega=0.3$ than for $\Omega=1.2$, given that in the former case the circulation becomes unstable at a much larger Reynolds number. For the case with $\Omega=1.2$ the solution features small barotropic recirculations, which however are strong enough so that to distort the geostrophic contours and create a small region of closed geostrophic contours in the western part of the basin. For the case with $\Omega=0.3$, again a region 
A)

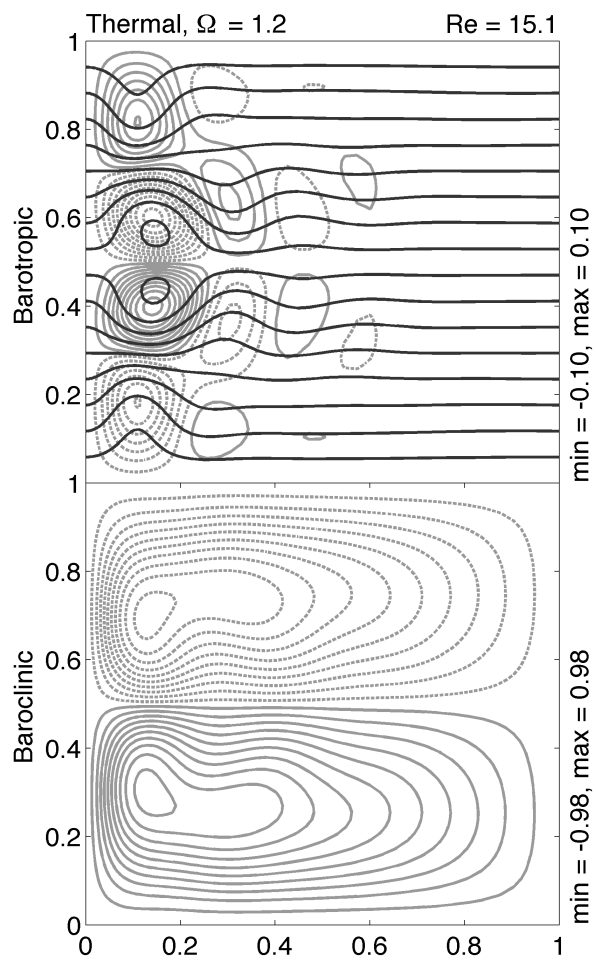

B)

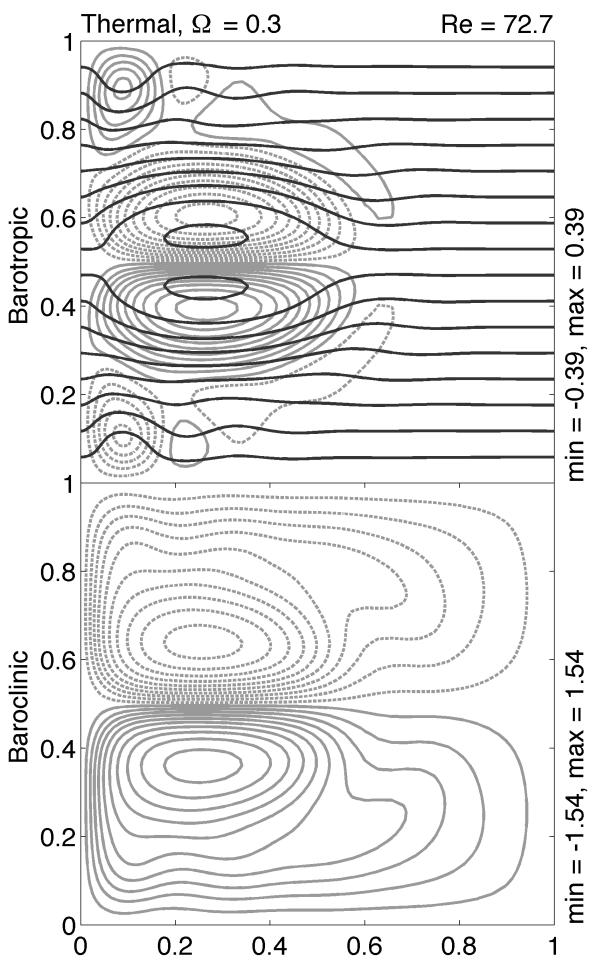

Figure 6-4: Barotropic and baroclinic streamfunction for a thermally-forced ocean at the critical Reynolds number, where the flow becomes unstable for the case with A) $\Omega=1.2\left(R_{d}=28.8 \mathrm{~km}\right)$, and B) $\Omega=0.3\left(R_{d}=57.7 \mathrm{~km}\right)$. Overlaid in gray on top of the barotropic streamfunction are the geostrophic contours $\hat{\phi}=y+\Omega \phi$. The value of the critical Reynolds number is indicated in each plot.

of closed geostrophic contours is present, due to the fact that the solution is more nonlinear $\left(R e_{H_{1}}=73\right)$ and the barotropic recirculations are stronger in magnitude.

For both values of the $\Omega$ parameter the first two perturbations to which the circulation becomes unstable are oscillating perturbations and are marked with the points $H_{1}$ and $H_{2}$ in Figure 6-3. The properties of the temporal variability that arises because of these oscillating perturbations are quite different for the two values of $\Omega$ and are analyzed next. 
A

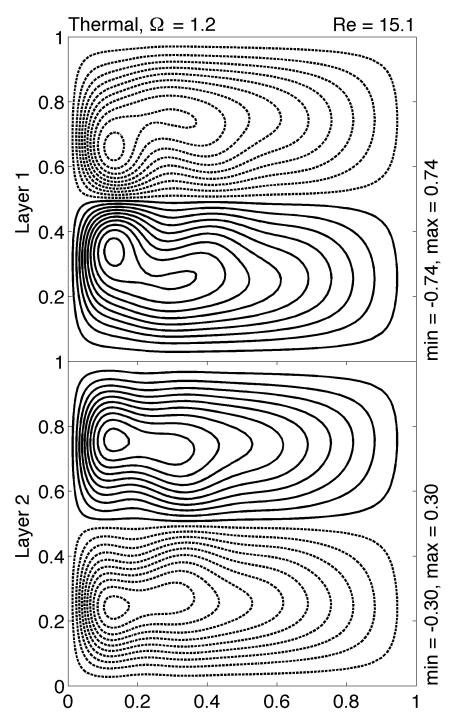

B

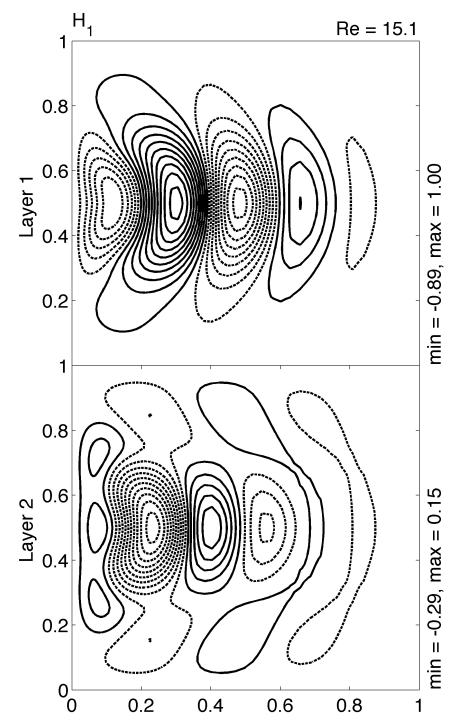

C

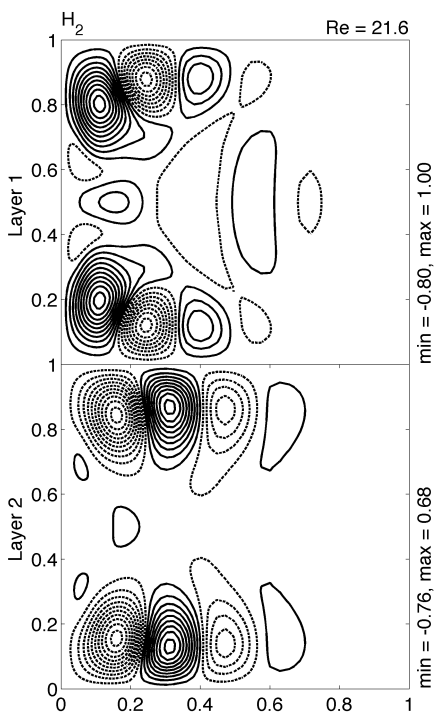

Figure 6-5: Equilibrium solution (A) and first two destabilizing perturbations (B and C) for the case of a thermally-forced ocean with $\Omega=1.2$. The perturbations are plotted at time $t=0$. Their periods are $T_{H_{1}} \approx 9$ months and $T_{H_{2}} \approx 5$ months.

\subsubsection{Onset of time-dependence for $\Omega=1.2$}

For $\Omega=1.2$ or the weakly stratified ocean with $R_{d}=28.8 \mathrm{~km}$, the oscillating perturbations $H_{1}$ and $H_{2}$ have a localized spatial structure and inter-monthly periods of respectively $T_{H_{1}} \approx 9$ months and $T_{H_{2}} \approx 5$ months.

In Figure 6-5 the perturbation streamfunctions at time $t=0$ are plotted by density layers, together with the equilibrium solution at the critical Reynolds number $R e_{H_{1}}$, where the flow becomes unstable. The equilibrium solution at the critical Reynolds number for the second perturbation $R e_{H_{2}}$ is not plotted, since the flow changes little between $R e_{H_{1}}$ and $R e_{H_{2}}$. Because the perturbations $H_{1}$ and $H_{2}$ are oscillating, more details about their temporal variability are shown in Figures 6-6 and 6-7, where the perturbation flow is plotted at four different phases during the oscillation. Note that the amplitude of the perturbation flow is arbitrary. When plotting, we have made the choice to scale the complex eigenvector so that the maximum of the layer 1 streamfunction at time $t=0$ be one, i.e $\max \left(\operatorname{Real}\left(\hat{\psi}_{1}(x, y),\right)\right)=1$.

The first destabilizing perturbation occurring at $R e_{H_{1}}=15.1$ is symmetric in the 

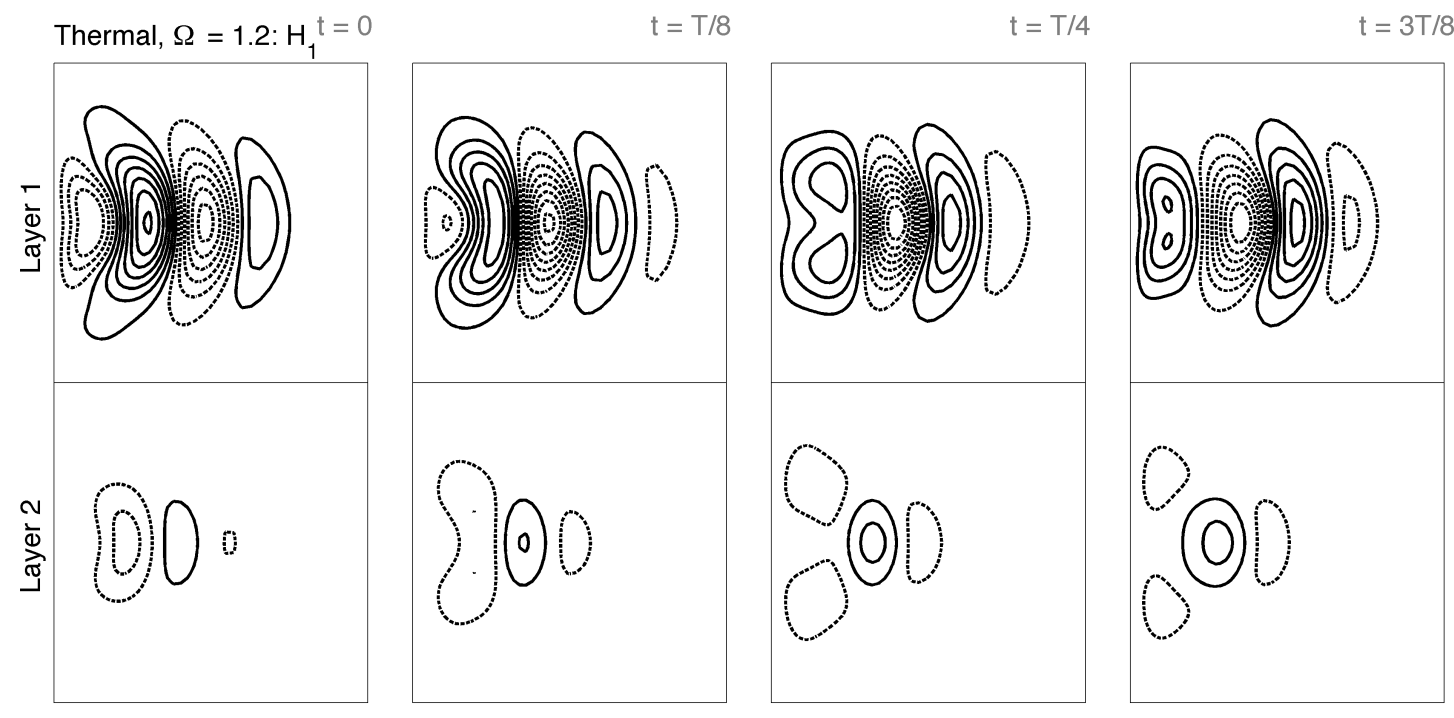

Figure 6-6: Perturbation $H_{1}$ ( $T_{H_{1}} \approx 9$ months) for the thermally-forced ocean with $\Omega=1.2$ plotted at 4 phases during the oscillation. The contour interval is 0.15 for both layers.
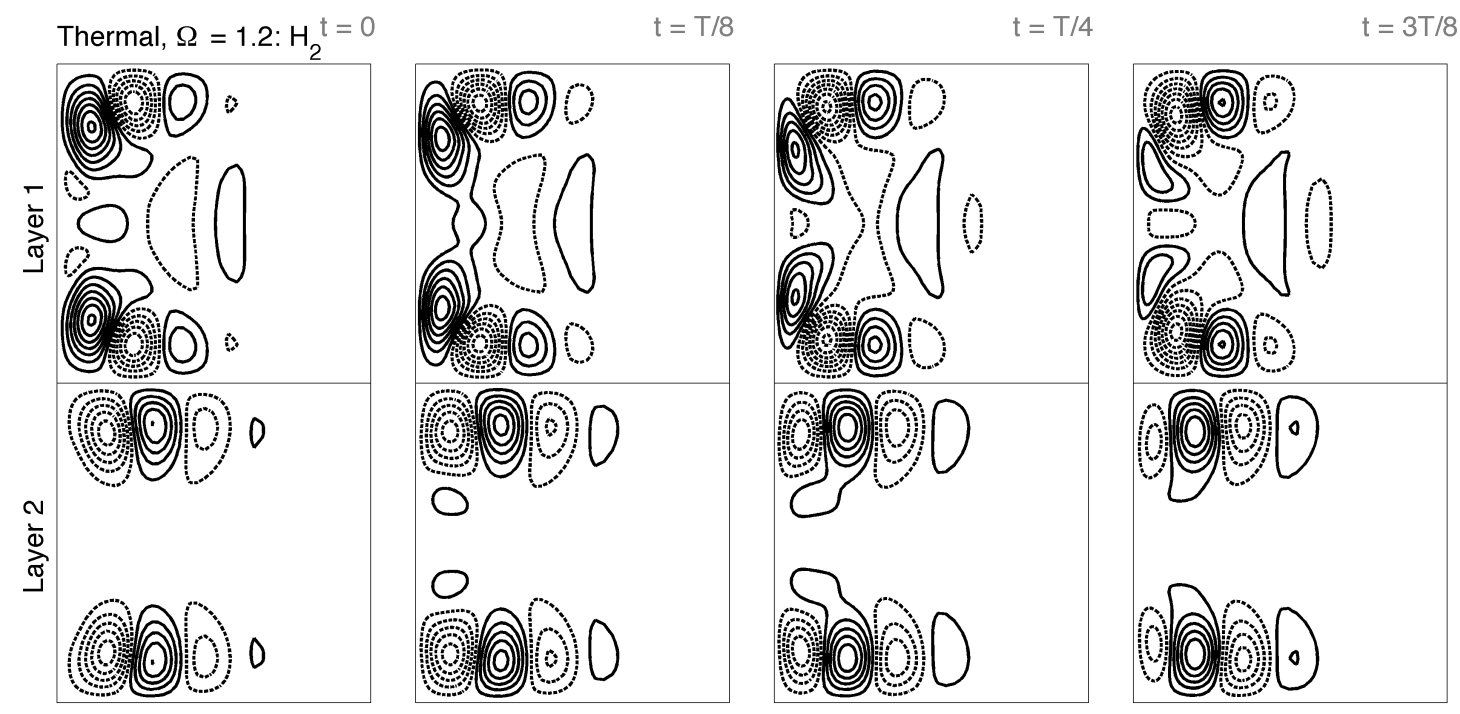

Figure 6-7: Perturbation $H_{2}\left(T_{H_{2}} \approx 5\right.$ months) for the thermally-forced ocean with $\Omega=1.2$ plotted at 4 phases during the oscillation. The contour interval is 0.15 for both layers.

meridional direction and aligned with the midlatitude zonal jet. It consists of alternating lows and highs that propagate westward. The mode has a zonal wavelength of approximatively $300 \mathrm{~km}$. A perturbation with similar structure has been shown 


\begin{tabular}{||l||r|r|r||r|r||}
\hline \hline \multirow{2}{*}{ Perturbation energy budget } & \multicolumn{3}{c||}{ Thermal, $\Omega=1.2$} & \multicolumn{2}{c||}{ Thermal, $\Omega=0.3$} \\
\cline { 2 - 6 } & $H_{1}$ & \multicolumn{2}{c||}{$H_{2}$} & $H_{1}$ & $H_{2}$ \\
\hline \hline $\mathrm{BT}_{1}+\mathrm{BT}_{2}$ & -43.37 & -1.02 & -2.15 & $\mathbf{3 0 . 9 5}$ & $\mathbf{2 7 . 5 7}$ \\
\hline $\mathrm{BC}$ & 0.73 & -0.02 & -0.08 & 1.15 & 1.49 \\
$\mathrm{P}-\mathrm{REL}=\iint d_{n} \hat{\psi}_{n}^{*} J\left(\hat{\psi}_{n}, \nabla^{2} \Psi_{n}\right)$ & 8.25 & -0.15 & -0.67 & 0.31 & 0.25 \\
$\mathrm{P}-\mathrm{STR}=\iint \alpha_{n} \hat{\psi}_{n}^{*} J\left(\hat{\psi}_{n}, \Psi_{1}-\Psi_{2}\right)$ & 4.16 & 0.67 & 2.82 & 2.42 & 1.45 \\
$\mathrm{P}-\mathrm{BETA}=\iint d_{n} \hat{\psi}_{n}^{*} J\left(\hat{\psi}_{n}, \beta y\right)$ & -360.55 & -103.98 & -446.99 & -58.52 & -46.20 \\
\hline $\mathrm{DISS}=\iint \frac{\mathbf{1 0 9 . 2 0}}{d_{n}} \hat{\psi}_{n}^{*} \nabla^{4} \hat{\psi}_{n}$ & -36.67 & -3.70 & -15.43 & -3.55 & -2.09 \\
\hline $\mathrm{REST}=\iint d_{1} d_{2} \frac{\beta}{\delta_{T}}\left|\hat{\psi}_{1}-\hat{\psi}_{2}\right|^{2}$ & $\mathbf{1 . 0 0}$ & $\mathbf{1 . 0 0}$ & $\mathbf{1 . 0 0}$ & $\mathbf{1 . 0 0}$ & $\mathbf{1 . 0 0}$ \\
\hline \hline$\sigma_{r} E$
\end{tabular}

Table 6.2: Perturbation energy budget integrated over the basin for the case of a thermally-forced ocean. All integrals are as described in Eq.(6.10) with summation over the two layers assumed, when applicable. The short notation $\alpha_{n}=(-1)^{n} d_{1} d_{2} F$ is used in the definition of the P-STR term. Since the amplitude of the perturbation is arbitrary, all terms have been rescaled so that $\sigma_{r} E=1$. The three integrals P-REL, P-STR, P-BETA are zero because of the no-normal flow boundary conditions. The source terms sustaining the growth are shown in bold.

to be the first unstable eigenmode in the case of a wind-driven double-gyre flow as well, except that in the wind-driven case the mode propagates eastward (Dijkstra and Katsman, 1997). When added to the equilibrium solution, the mode causes the free zonal jet to meander. The period of the temporal variability is $T_{H_{1}} \approx 9$ months.

At the point $H_{2}$, not one but actually two pairs of eigenvalues cross the real axis. Since their corresponding eigenvectors have very similar spatial structures and nearly identical periods, only one is shown in Figures 6-5 and 6-7. These oscillating perturbations are confined to the zonal boundary layers along the southern and northern walls. They consist of cells of opposite direction motion propagating westward. The zonal wavelength of the perturbations is again on the order of $300 \mathrm{~km}$. When added to the equilibrium solution, they lead to oscillation of the zonal boundary layers with period $T_{H_{2}} \approx 5$ months.

Examining the integrated energy budget for these perturbations can help us iden- 
A

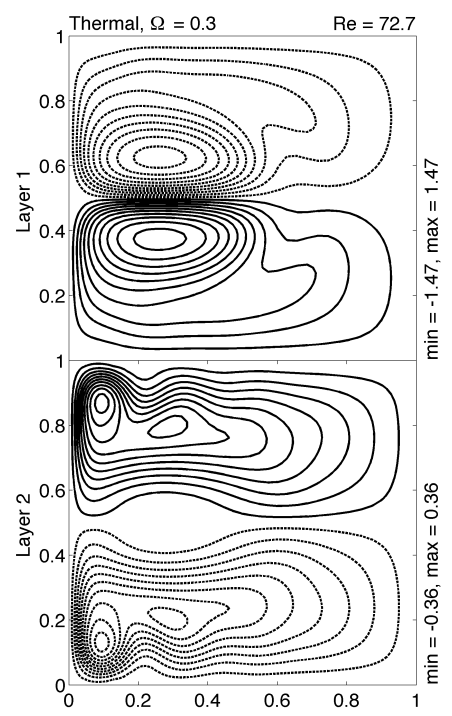

$\mathrm{B}$

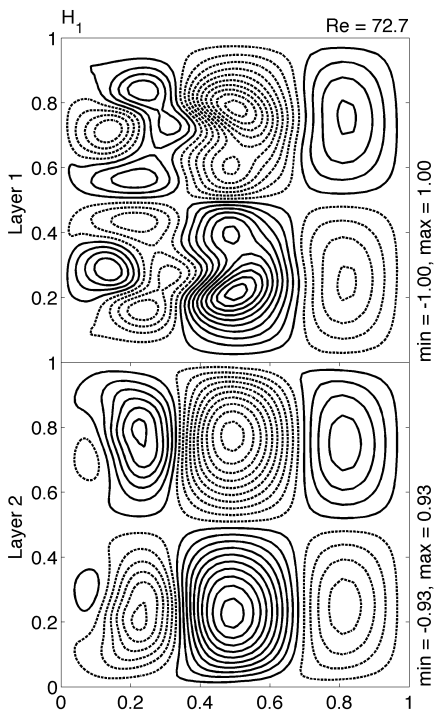

C

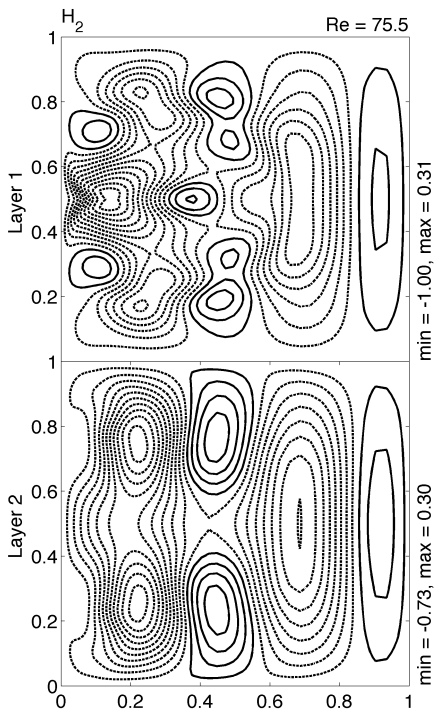

Figure 6-8: Equilibrium solution (A) and first two destabilizing perturbations (B and C) for the case of a thermally-forced ocean with $\Omega=0.3$. The perturbations are plotted at time $t=0$. Their periods are $T_{H_{1}} \approx 54.0$ days and $T_{H_{2}} \approx 53.6$ days.

tify the instability process responsible for their growth. The details of the integrated perturbation energy budget are shown in Table 6.2. For all modes, from the two possible perturbation energy sources only the baroclinic conversion term integrated over the basin is positive, while the barotropic conversion term as well as the dissipation are both negative. Thus, in the case of $\Omega=1.2$ or the weakly stratified ocean with $R_{d}=28.8 \mathrm{~km}$, the oscillating perturbations are generated by a baroclinic instability of the flow.

\subsubsection{Onset of time-dependence for $\Omega=0.3$}

Different type of temporal variability appears in the case of a thermally-forced ocean with $\Omega=0.3$, or the strongly stratified ocean with $R_{d}=57.7 \mathrm{~km}$. In this case, the oscillating perturbations $H_{1}$ and $H_{2}$ have a basin-scale spatial structure and shorter periods of respectively $T_{H_{1}} \approx 54.0$ days and $T_{H_{2}} \approx 53.6$ days, i.e slightly less than 2 months.

In Figure 6-8, the first two oscillating perturbations at time $t=0$, together with 

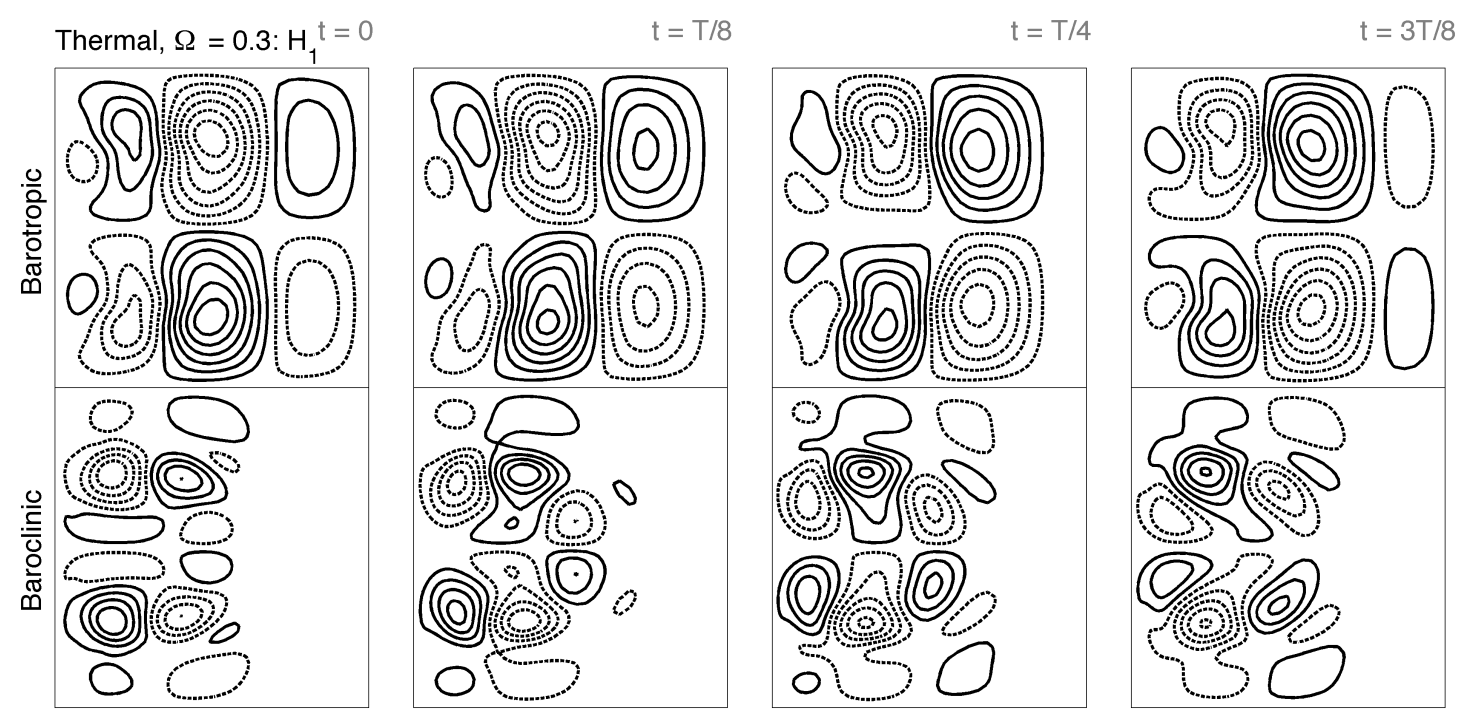

Figure 6-9: Perturbation $H_{1}\left(T_{H_{1}} \approx 54.0\right.$ days) for the thermally-forced ocean with $\Omega=0.3$ plotted at 4 phases during the oscillation. The contour interval is 0.15 for both vertical modes.
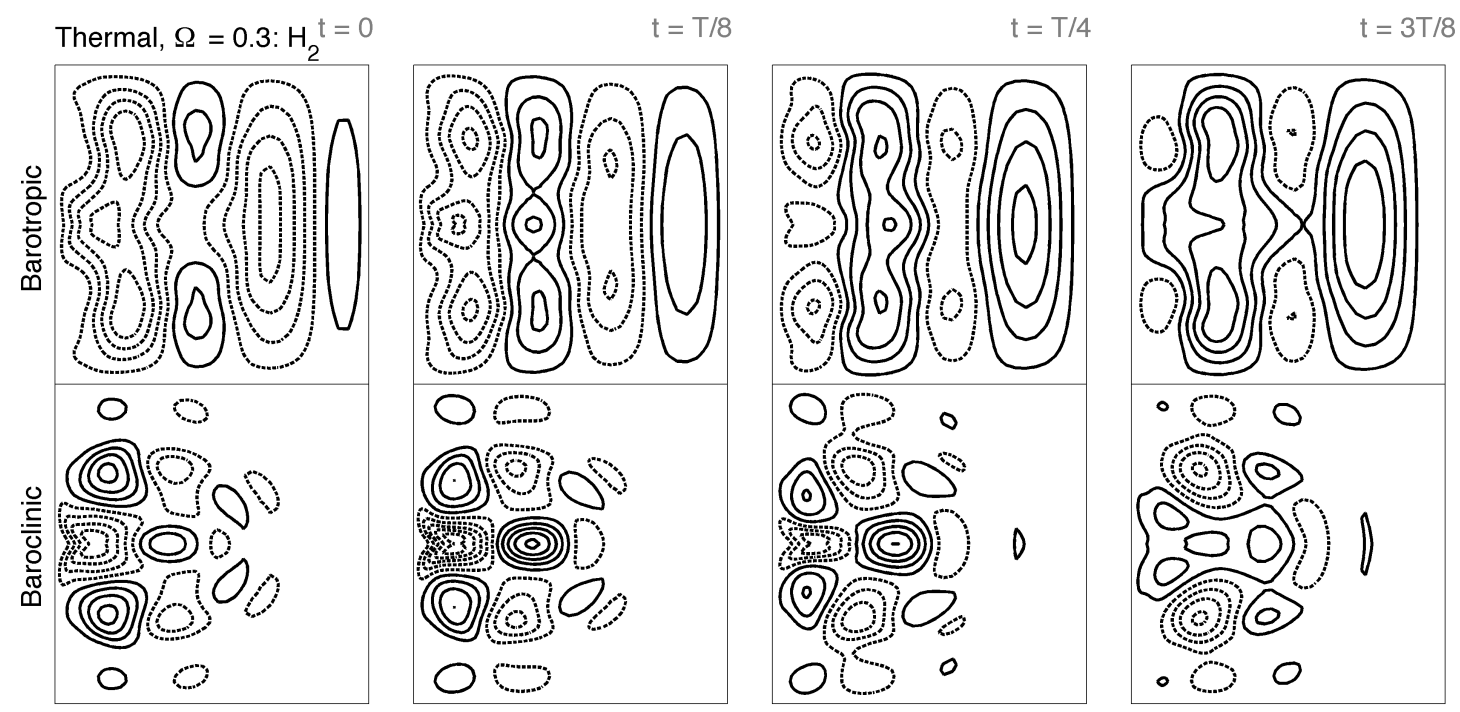

Figure 6-10: Perturbation $H_{2}\left(T_{H_{2}} \approx 53.6\right.$ days $)$ for the thermally-forced ocean with $\Omega=0.3$ plotted at 4 phases during the oscillation. The contour interval is 0.15 for both vertical modes.

the equilibrium solution at the critical Reynolds number $R e_{H_{1}}$ are plotted. Both points $H_{1}$ and $H_{2}$ correspond in this case to the crossing of the real axis by a single pair of eigenvalues. In order to underline the different nature of the temporal vari- 
ability generated by these eigenmodes, we have plotted in Figures 6-9 and 6-10 the perturbation flow by vertical modes, and not by density layers, at 4 different phases during the oscillation. For both eigenmodes, the barotropic part of the perturbation flow bears similarity to a barotropic Rossby basin mode. If the complex eigenvector is plotted instead in the form of a spatial phase and a spatial amplitude (not shown here), it can be seen that the perturbations have a clear westward phase propagation and a spatial amplitude consistent with a $1 \times 2$ and a $2 \times 1$ Rossby basin mode, for the $H_{1}$ and $H_{2}$ perturbation, respectively. The theoretical period for a $1 \times 2$ or $2 \times 1$ Rossby basin mode in a basin of size $L=1000 \mathrm{~km}$ and planetary vorticity gradient $\beta_{0}=2 \times 10^{-11} 1 / m s$ is

$$
T_{12}=\frac{4 \pi^{2}}{\beta_{0} L} \sqrt{n^{2}+m^{2}} \approx 51.2 \text { days }
$$

which is close to the period of the two perturbations.

Therefore, in the case with $\Omega=0.3$ the thermally-forced double-gyre flow becomes unstable to perturbations that are similar to barotropic Rossby basin modes. They are not exactly basin modes since their periods do not quite match those of the theoretical basin modes and the pattern of variability is somewhat distorted in the western part of the basin where the advective terms become important. The baroclinic part of the variability in Figures 6-9 and 6-10 represents simply the advection by the corresponding barotropic perturbation flow of the equilibrium interface height. This is similar to what was found in the strongly nonlinear regime of circulation characterized by large amplitude basin modes presented in the previous chapter.

There are some distinct differences concerning the source of the instability as well. The details of the integrated perturbation energy budget for the case with $\Omega=0.3$ is shown in Table 6.2. Unlike the case with $\Omega=1.2$, both the barotropic and the baroclinic energy conversion terms integrated over the basin are positive. Thus, in the case of $\Omega=0.3$ or the strongly stratified ocean with $R_{d}=57.7 \mathrm{~km}$, the oscillating perturbations arise because of a mixed barotropic-baroclinic instability of the flow. 


\subsubsection{Hypothesis}

The stability analysis of the thermally-forced double-gyre flow showed that the type of temporal variability that arises and the instability that leads to it, depend on the value of the $\Omega$ parameter, where $\Omega$ is equal to the ratio of the flow speed $U$ to the speed of a long internal Rossby wave $\beta_{0} R_{d}^{2}$. When all other parameter are fixed, for large values of $\Omega$ the most unstable perturbations have a spatially localized structure, inter-monthly periods and are generated by a baroclinic instability of the equilibrium state. For small values of $\Omega$, on the other hand, the destabilizing perturbations are due to a mixed barotropic-baroclinic instability and resemble closely barotropic Rossby basin modes - they are characterized with a basin-scale spatial structure, westward phase propagation and monthly periods. A result that we did not show here, is that even below the critical Reynolds number when the circulation is linearly stable, there is again the same distinction between the most unstable eigenmodes as a function of $\Omega$. For large values of $\Omega$, the least stable eigenmode (i.e that decays the slowest) is again a localized, baroclinic type of perturbation, while for small values of $\Omega$, the least stable eigenmode is a basin mode.

The hypothesis that we would like to put forward is that the type of variability that appears, or in other words the criterium that determines what is a large and what is a small value for $\Omega$, is the geometry of the geostrophic contours, $\hat{\phi}=y+\Omega \phi$ in the limit of small Reynolds number. The larger the value for $\Omega$, the easier it is to get a region of closed geostrophic contours, isolated from the eastern wall. Our hypothesis is that when such a region of closed geostrophic contours is present, the onset of time-dependence happens through modes that are spatially localized, either to the zonal jet region or to the zonal boundary layers. If on the other hand the geostrophic contours are blocked and only slightly distorted, then the temporal variability that arises resembles barotropic Rossby basin modes. From the calculations performed so far, it seems that it is enough to determine the geometry of geostrophic contour in the nearly linear limit (not at the critical Reynolds number) in order to be able to predict the type of time-dependence to appear in the system. 
In order to test this hypothesis, we will examine next the onset of time-dependence in a wind-driven double-gyre circulation.

\subsection{Stability of a wind-forced double-gyre flow}

Because in a thermally-forced ocean the barotropic circulation is generated through the nonlinear coupling to the baroclinic circulation, and there is no barotropic circulation at all in the linear limit, it is difficult to predict a priori for what values of $\Omega$ closed geostrophic contours are expected. In order to test our hypothesis that the type of temporal variability that arises in a system depends on the geometry of the geostrophic contours we will examine in this section the onset of time-dependence in a wind-forced double-gyre flow. It is not expected that the type of forcing that drives the equilibrium state changes the general stability properties of the circulation. We anticipate thus to find a similar dependence on $\Omega$ of the instabilities that occurs in terms of their spatial characteristics, oscillation periods and energy sources, as the one determined for the thermally-forced double-gyre flow. The stability of the winddriven double-gyre circulation has been examined previously on several occasions (Dijkstra and Katsman, 1997; Nauw et al., 2004; Primeau, 1998; Simonnet, 2005). In

particular, Dijkstra and Katsman (1997) study the onset of temporal variability in a 2-layer wind-driven QG model identical to ours. However, the specific question of how the circulation stability depends on the $\Omega$ parameter, or equivalently the Froude number $F$, has not been fully examined in any of the previous studies.

\subsubsection{Model setup}

When the ocean is driven by wind forcing, we can determine the barotropic circulation in the low Reynolds number limit from a simple Sverdrup balance

$$
\beta \frac{\partial \phi}{\partial x}=\frac{H_{1}}{H} U_{w} F_{w}(y)
$$




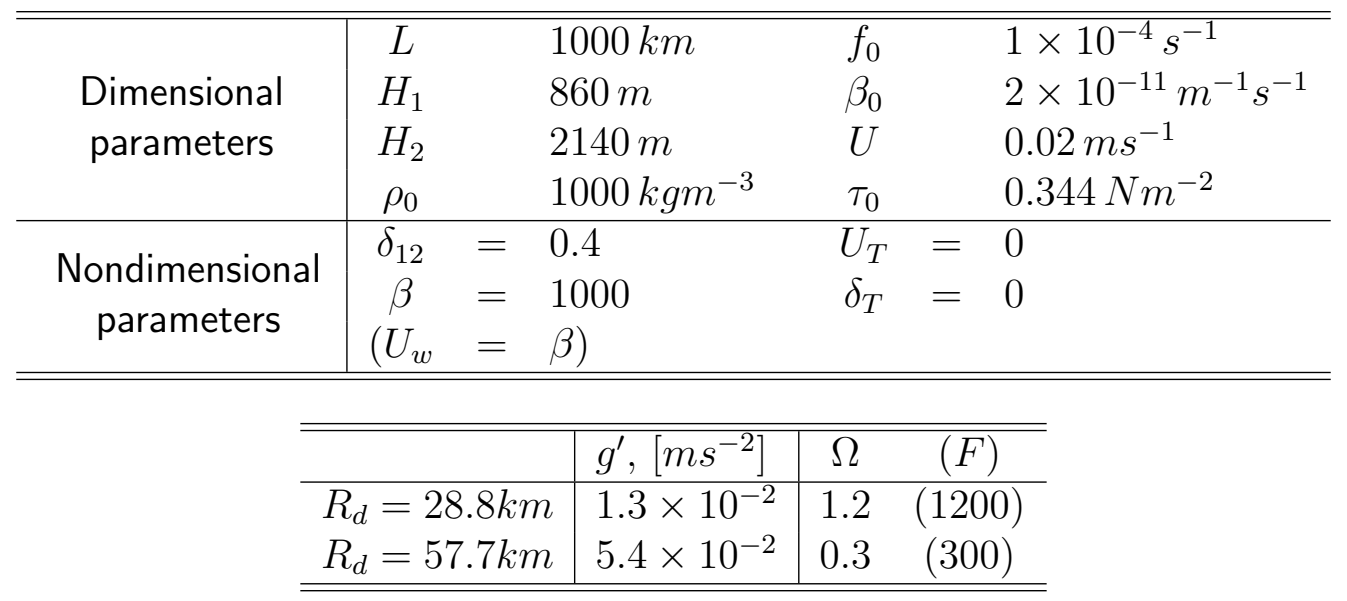

Table 6.3: Dimensional and nondimensional parameters used for the wind-forced stability calculations. Two different values of the internal deformation radius are applied, leading to two different values for the $\Omega$ parameter, or alternatively the Froude number $F$. The Reynolds number is not specified, since it is used as a control parameter.

A)

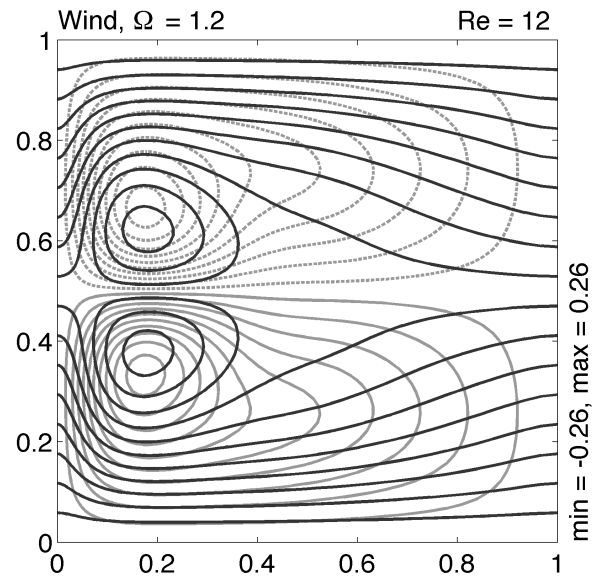

B)

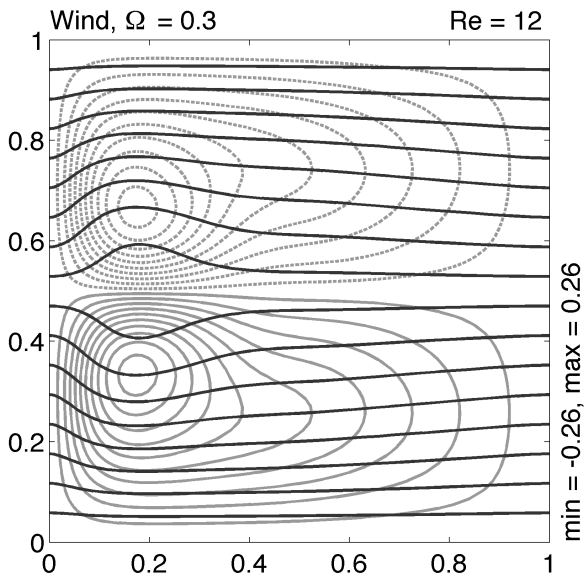

Figure 6-11: Barotropic circulation in gray and geostrophic contours $\hat{\phi}=y+\Omega \phi$ in black for a wind-forced ocean with A) $\Omega=1.2\left(R_{d}=28.8 \mathrm{~km}\right)$, and B) $\Omega=0.3$ $\left(R_{d}=57.7 \mathrm{~km}\right)$. In both cases, $R e=12$ is used and the resulting circulation is steady.

Based on this linear estimate for the vertically integrated circulation $\phi$, it is possible to determine a critical value for the parameter $\Omega=\Omega_{c}$, above which closed geostrophic contours are expected. It can be shown that this value is

$$
\Omega_{c}=\frac{1}{\pi} \frac{H}{H_{1}}=\frac{1+\delta_{12}}{\pi \delta_{12}}
$$


which, for the geometry of our model where $\delta_{12}=0.4$, leads to $\Omega_{c}=1.1$.

Guided by this value, we have chosen to perform stability calculations on two wind-driven configurations - one with $\Omega=1.2>\Omega_{c}$, and one with $\Omega=0.3<\Omega_{c}$. These are, not by coincidence, the same values as the ones used for the thermallyforced ocean. Actually, all nondimensional parameters have been kept the same as in the thermally-foced stability calculations in order to be able to draw comparisons between the two cases. The full list of parameters used in the wind-driven calculations are listed in Table 6.3. Note also that the configuration with $\Omega=1.2$ or $F=1200$ corresponds to the set of parameters used in Dijkstra and Katsman (1997). The only difference from their model setup is the applied boundary conditions - they use free-slip conditions on the northern and southern walls and no-slip conditions on the eastern and western walls, while we use no-slip conditions everywhere.

In Figure 6-11 the barotropic streamfunction at Reynolds number $R e=12$, where the circulation is steady for both values of $\Omega$, is plotted and overlaid with the geostrophic contours, $\hat{\phi}=y+\Omega \phi$. Note that for a wind-driven ocean the equilibrium solutions are independent of $\Omega$, i.e the barotropic streamfunctions plotted in Figure 6-11 A) and B) are identical. The reason for this is that the wind stress sets in motion only the upper layer, while the lower layer remains motionless, i.e $\Psi_{2}=0$. Consequently, the advection of stretching vorticity, which is proportional to $\Omega J\left(\Psi_{1}, \Psi_{2}\right)$, is identically zero. Given that this is the only term through which the $\Omega$ parameter participates in the equations, the stationary solutions of a wind-driven model are independent of the value of $\Omega$. The form of the geostrophic contours however, as well as the stability of the equilibrium solutions depend on $\Omega$.

In Figure 6-11, it can be seen that for the same barotropic circulation, the geostrophic contours are all blocked and nearly zonal for $\Omega=0.3$ (or $R_{d}=57.7 \mathrm{~km}$ ) when the Rossby waves are fast, while there is a distinct region of closed geostrophic contours for $\Omega=1.2$ (or $R_{d}=28.8 \mathrm{~km}$ ) when the Rossby waves are slow enough so that their westward propagation can be arrested by the barotropic flow.

In order to determine the threshold for instability of the circulation we have fol- 

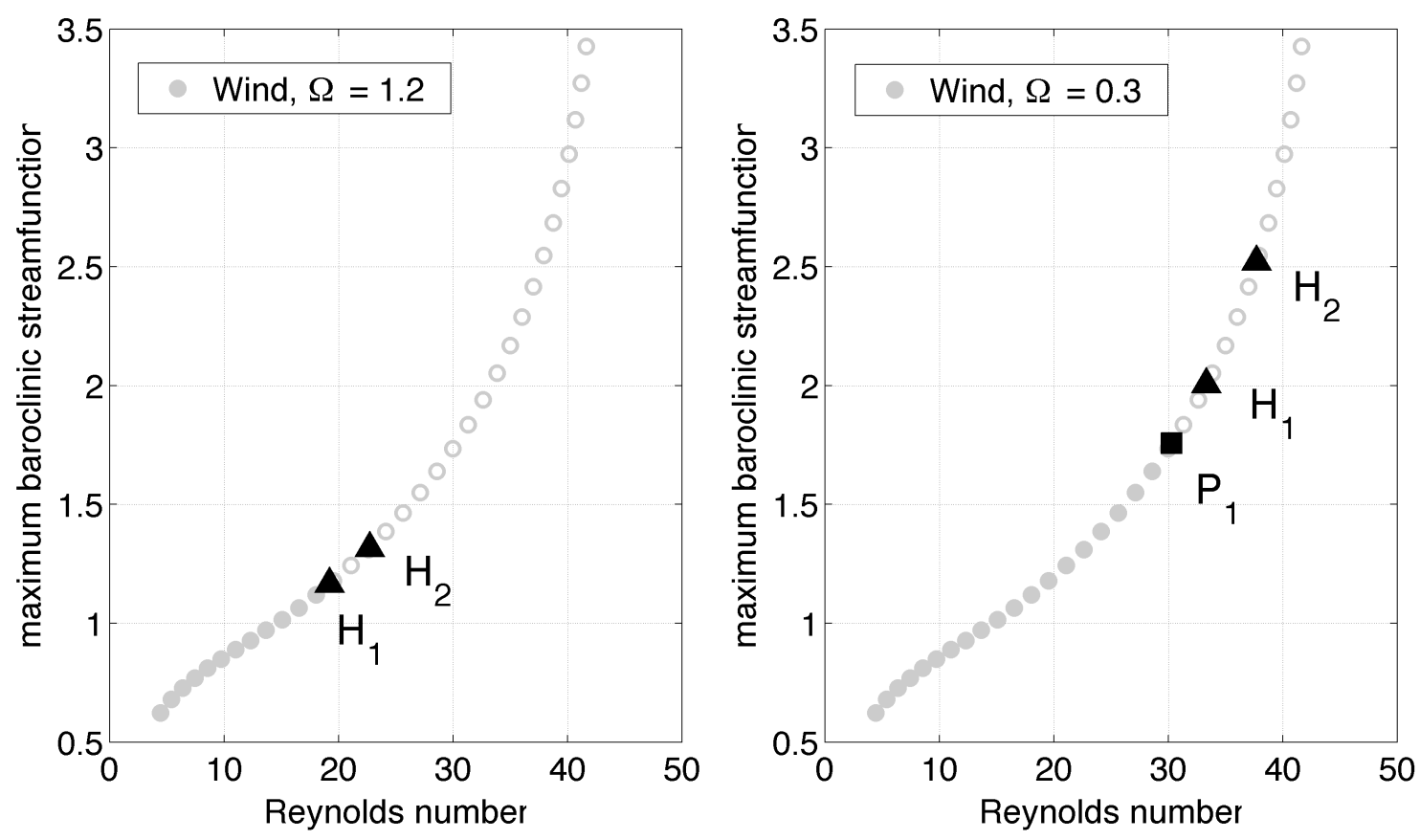

Figure 6-12: Maximum of the baroclinic streamfunction as a function of the Reynolds number for $\Omega=1.2$ (left) and $\Omega=0.3$ (right) in the case of a wind-forced ocean. Filled symbols indicate stable equilibrium states, for which all eigenvalues have negative real parts. Empty symbols indicate unstable equilibrium states, for which there is at least one eigenvalue with positive real part. The approximative Reynolds numbers at which an eigenvalue crosses the real axis are shown either in triangles and denoted by $H_{n}$, which stands for an instability to an oscillating perturbation (Hopf bifurcation), or in squares and denoted by $P_{n}$, which stands for an instability to a stationary perturbation (Pitchfork bifurcation).

lowed the stationary solutions when the Reynolds number is increased. In Figure 6-12 the norm of the equilibrium solution, as represented by the the maximum of the baroclinic streamfunction, is plotted as a function of the Reynolds number. In the wind-driven case, the curves for $\Omega=1.2$ and $\Omega=0.3$ are identical but the critical Reynolds numbers are different. The circulation corresponding to $\Omega=1.2$, which can be thought of as the weakly stratified configuration, becomes unstable at a lower Reynolds number: $R e_{H_{1}}=19.3$ for $\Omega=1.2$, compared to $R e_{P_{1}}=30.4$ for $\Omega=0.3$. In the later case, the circulation becomes actually first unstable to a stationary perturbation, denoted by $P_{1}$. That means that the instability does not lead to temporal 

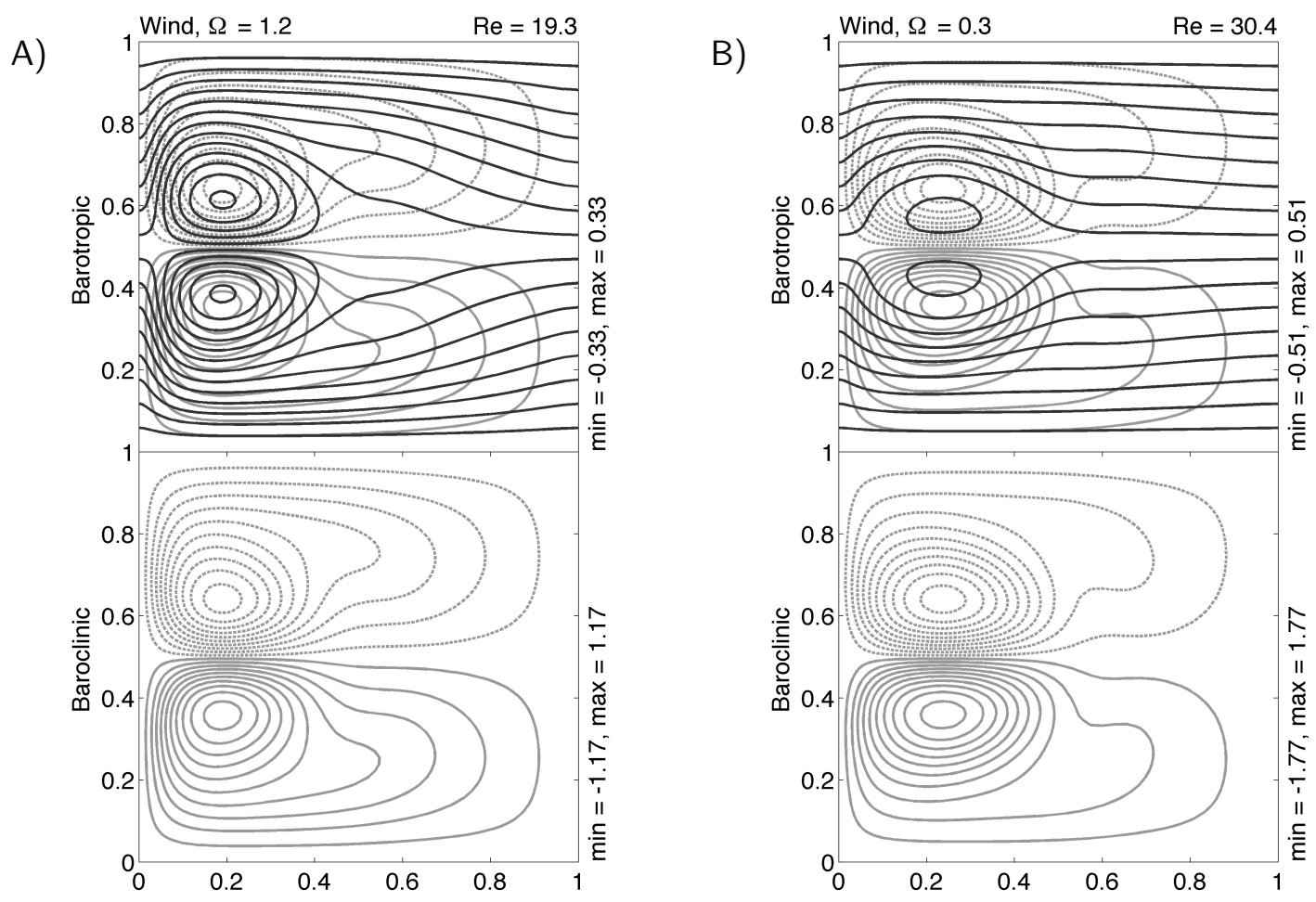

Figure 6-13: Barotropic and baroclinic streamfunction for a wind-forced ocean at the critical Reynolds number, where the flow becomes unstable for the case with A) $\Omega=1.2\left(R_{d}=28.8 \mathrm{~km}\right)$, and B) $\Omega=0.3\left(R_{d}=57.7 \mathrm{~km}\right)$. Overlaid in gray on top of the barotropic streamfunction are the geostrophic contours $\hat{\phi}=y+\Omega \phi$. The value of the critical Reynolds number is indicated in each plot.

variability but instead multiple stationary solutions are created. The circulation becomes unstable to an oscillatory perturbation at a slightly larger Reynolds number, $R e_{H_{1}}=33.3$.

In Figure 6-13 the pattern of the circulation by vertical modes at the critical Reynolds number for both values of $\Omega$ is plotted. Given that the circulation for $\Omega=0.3$ destabilizes at a slightly larger Reynolds number and is thus more nonlinear, the streamfunction is of larger magnitude and the recirculations, slightly larger in size. Despite that, a distinct region of closed geostrophic contours is evident for $\Omega=1.2$, while they are mostly zonal with only a few closed contours for $\Omega=0.3$. The closed contours in the later case form because of the nonlinear advection - they were absent in the linear limit given that $\Omega=0.3<\Omega_{c}$ (Figure 6-11). Therefore, at least in what 
A

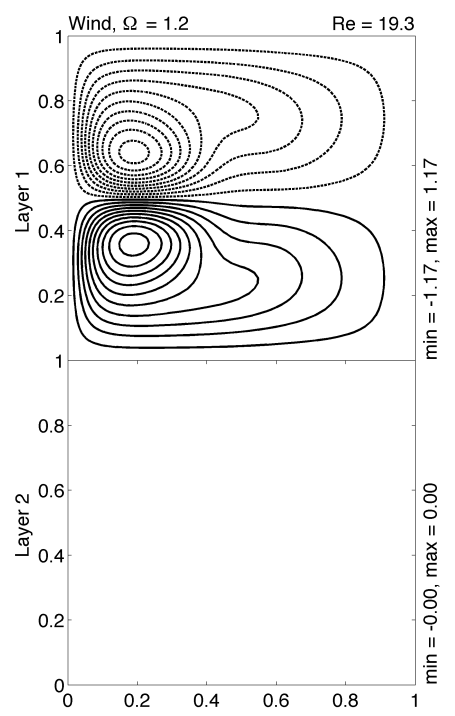

B

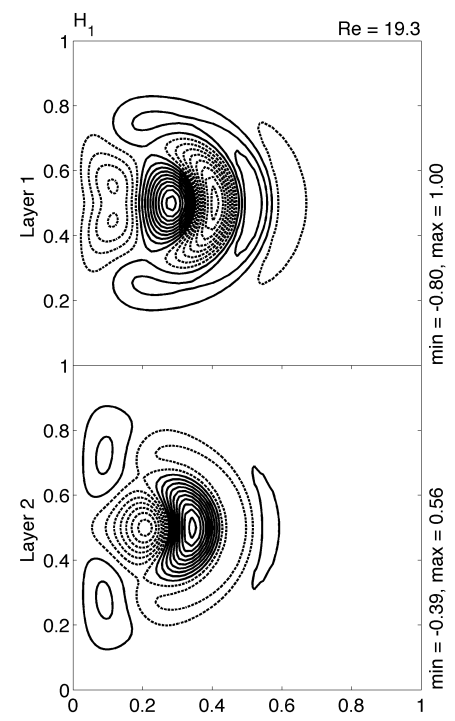

C

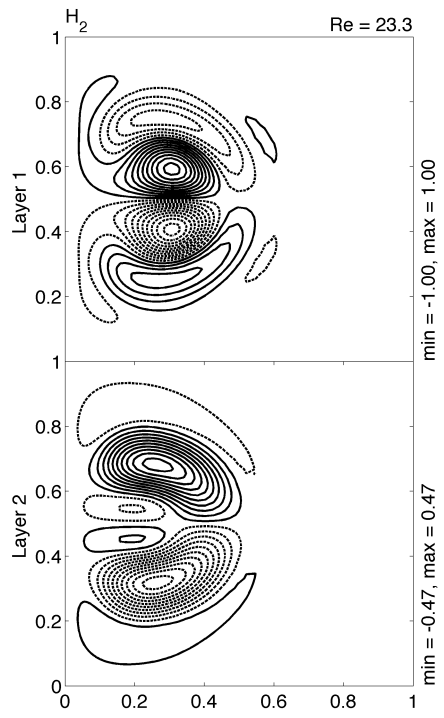

Figure 6-14: Equilibrium solution (A) and first two destabilizing perturbations (B and $\mathrm{C}$ ) for the case of a wind-forced ocean with $\Omega=1.2$. The perturbations are plotted at time $t=0$. Their periods are $T_{H_{1}} \approx 3.8$ months and $T_{H_{2}} \approx 6.4$ months.

concerns the threshold of instability and the geometry of the geostrophic contours at the critical Reynolds number, the wind-forced circulation behaves similarly to the thermally-forced one. The one difference that we found so far is the presence of the stationary instability $P_{1}$ for the $\Omega=1.2$ wind-driven case.

What is of further interest to us in order to test our hypothesis is to examine the properties of the destabilizing oscillatory perturbations.

\subsubsection{Onset of time-dependence for $\Omega=1.2$}

The wind-driven case with $\Omega=1.2$ has been previously studied by Dijkstra and Katsman (1997). Despite the slight differences in the applied boundary conditions we essentially reproduce part of their results. In Figure 6-14 the equilibrium solution at the critical Reynolds number together with the first two destabilizing perturbations are plotted by density layers. The perturbations are shown only at time $t=0$. The first destabilizing perturbation with critical Reynolds number $R e_{H_{1}}=19.3$ is very similar to the one determined for the thermally-forced ocean with $\Omega=1.2$. It is 


\begin{tabular}{||l||r|r||r|r|r||}
\hline \hline \multirow{2}{*}{ Perturbation energy budget } & \multicolumn{2}{|c||}{ Wind, $\Omega=1.2$} & \multicolumn{3}{c||}{ Wind, $\Omega=0.3$} \\
\cline { 2 - 6 } & $H_{1}$ & $H_{2}$ & $H_{1}$ & $H_{2}$ & $P_{1}$ \\
\hline \hline $\mathrm{BT}_{1}+\mathrm{BT}_{2}$ & -1059.13 & -7.98 & $\mathbf{2 5 . 2 0}$ & $\mathbf{1 . 8 1}$ & $\mathbf{3 5 1 . 7 1}$ \\
\hline $\mathrm{BC}$ & $\mathbf{3 6 4 2 . 5 0}$ & $\mathbf{4 3 . 6 8}$ & $\mathbf{1 0 7 . 9 7}$ & $\mathbf{1 2 . 7 8}$ & -3.75 \\
\hline $\mathrm{P}-\mathrm{REL}=\iint d_{n} \hat{\psi}_{n}^{*} J\left(\hat{\psi}_{n}, \nabla^{2} \Psi_{n}\right)$ & -9.79 & 0.05 & 0.36 & 0.05 & 4.29 \\
$\mathrm{P}-\mathrm{STR}=\iint \alpha_{n} \hat{\psi}_{n}^{*} J\left(\hat{\psi}_{n}, \Psi_{1}-\Psi_{2}\right)$ & -7.90 & 0.33 & 0.26 & 0.02 & 3.65 \\
$\mathrm{P}-\mathrm{BETA}=\iint d_{n} \hat{\psi}_{n}^{*} J\left(\hat{\psi}_{n}, \beta y\right)$ & 18.42 & 0.19 & 2.69 & 0.22 & 2.58 \\
\hline $\mathrm{DISS}=\iint \frac{d_{n}}{R e} \hat{\psi}_{n}^{*} \nabla^{4} \hat{\psi}_{n}$ & -2583.09 & -35.28 & -135.48 & -13.87 & -357.47 \\
\hline $\mathrm{REST}=\iint d_{1} d_{2} \frac{\beta}{\delta_{T}}\left|\hat{\psi}_{1}-\hat{\psi}_{2}\right|^{2}$ & - & - & - & - & - \\
\hline \hline$\sigma_{r} E$ & $\mathbf{1 . 0 0}$ & $\mathbf{1 . 0 0}$ & $\mathbf{1 . 0 0}$ & $\mathbf{1 . 0 0}$ & $\mathbf{1 . 0 0}$ \\
\hline \hline
\end{tabular}

Table 6.4: Perturbation energy budget integrated over the basin for the case of a wind-forced ocean. All integrals are as described in Eq.(6.10) with summation over the two layers assumed, when applicable. The short notation $\alpha_{n}=(-1)^{n} d_{1} d_{2} F$ is used in the definition of the P-STR term. Since the amplitude of the perturbation is arbitrary, all terms have been rescaled so that $\sigma_{r} E=1$. The three integrals P-REL, P-STR, P-BETA are zero because of the no-normal flow boundary conditions. The source terms sustaining the growth are shown in bold.

confined spatially to the midlatitude jet region and consists of alternating lows and highs propagating eastward. It causes meandering of the jet with period $T_{H_{1}}=$ 3.8 months.

The second destabilizing perturbation with critical Reynolds number $R e_{H_{2}}=23.3$ is different from the ones found in the thermally-forced ocean. It has however spatially localized structure and leads to temporal variability at inter-monthly periods, which is consistent with our hypothesis. The perturbation streamfunction is antisymmetric in the meridional direction and rotates anticlockwise/clockwise in the northern/southern half-basin (not shown here). It causes weakening and strengthening of the midlatitude jet and recirculations with period $T_{H_{2}}=6.4$ months .

The integrated perturbation energy budget for both modes is shown in Table 6.4. We find that for $\Omega=1.2$, similarly to the thermally-forced case, the first destabilizing perturbations are generated by a baroclinic instability of the equilibrium circulation. 
A

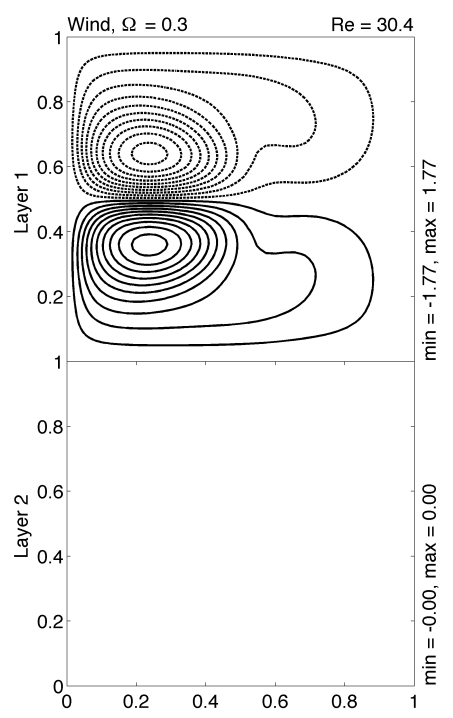

B

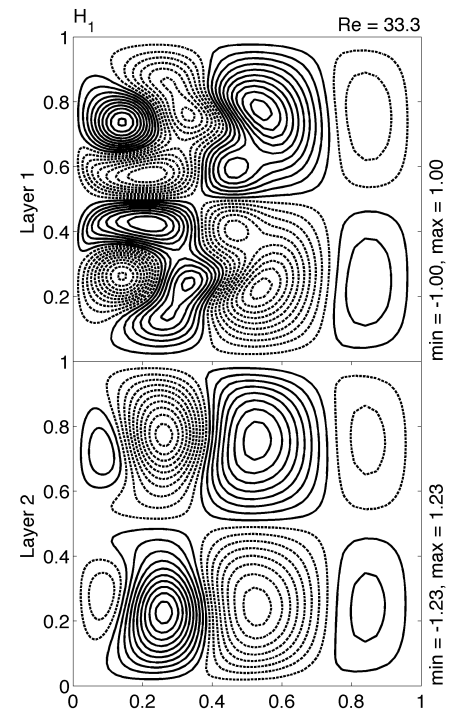

C

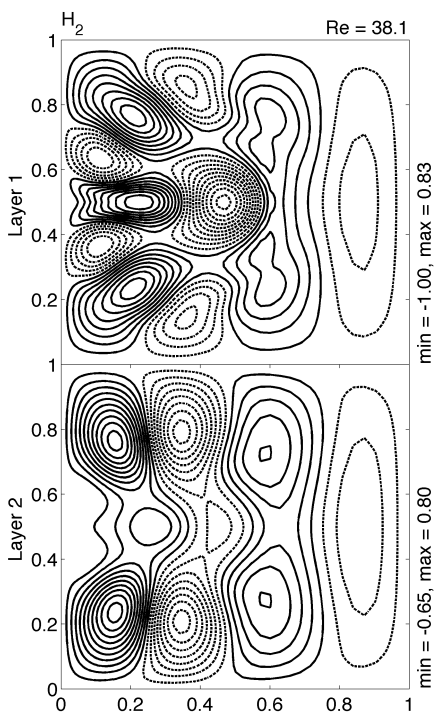

Figure 6-15: Equilibrium solution (A) and first two oscillatory perturbations (B and C) for the case of a wind-forced ocean with $\Omega=0.3$. The perturbations are plotted at time $t=0$. Their periods are $T_{H_{1}} \approx 53.5$ days and $T_{H_{2}} \approx 49.5$ days.

\subsubsection{Onset of time-dependence for $\Omega=0.3$}

As already noticed, the wind-driven circulation with $\Omega=0.3$ becomes first unstable at $R e_{P_{1}}=30.4$ to a stationary perturbation. However, because we are interested in the onset of time-dependence, we will look more closely at the next two perturbations to which the flow becomes unstable, which are both oscillatory. Their critical Reynolds numbers are $R e_{H_{1}}=33.3$ and $R e_{H_{2}}=38.1$, respectively.

In Figure 6-15 the equilibrium solution at the critical Reynolds number is plotted together with the first two oscillatory perturbations at time $t=0$. They both resemble very much the destabilizing perturbations for the thermally-forced case with $\Omega=0.3$. The perturbations $H_{1}$ and $H_{2}$ have a basin-scale spatial structure reminiscent of a $1 \times 2$ and a $2 \times 1$ barotropic Rossby basin mode, respectively. Their periods are close to the theoretical period for a basin mode of this order, $T_{1 \times 2}=51.2$ days. Similarly to the thermally-forced case, there is some distortion of the basin mode pattern in the western part of the basin. An integrated perturbation energy budget shows that both oscillatory modes in the wind-driven case with $\Omega=0.3$ are generated by a mixed 

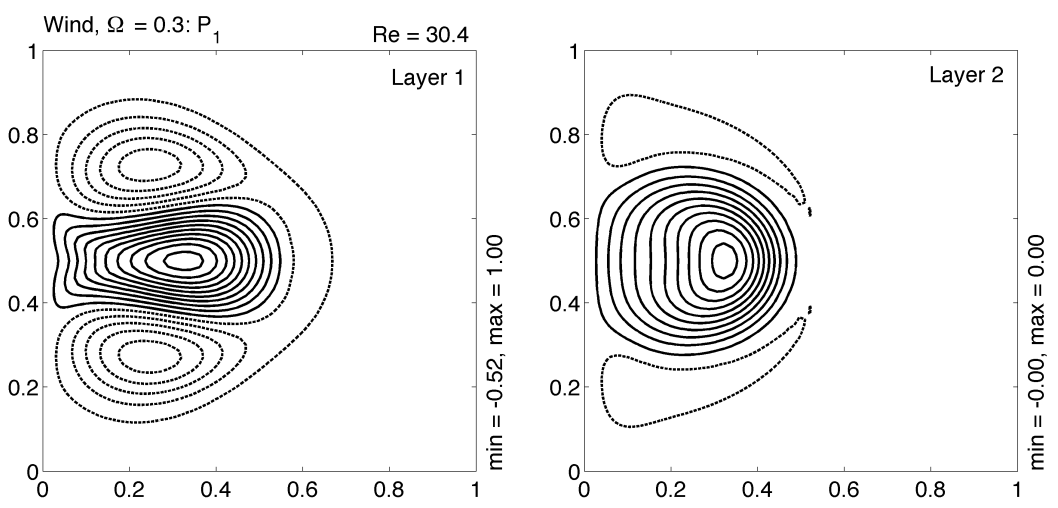

Figure 6-16: Streamfunction for the stationary perturbation $P_{1}$ to which the windforced ocean with $\Omega=0.3$ becomes linearly unstable at $R e_{P_{1}}=30.4$. Note that the amplitude in the lower layer is essentially zero.

barotropic-baroclinic instability of the equilibrium state.

For completeness, the stationary perturbation $P_{1}$ is plotted as well in Figure 616. Because this is a stationary perturbation, it is confined to the upper layer only (the amplitude in the lower layer is essentially zero). The streamfunction pattern is symmetric in the meridional direction. This instability causes the antisymmetric double-gyre flow state to become unstable while two other non-symmetric stable states are created - one with the jet deflected north, and one with the jet deflected south (Dijkstra and Katsman, 1997). Unlike all oscillatory perturbations examined in this chapter, the stationary perturbation is due to a barotropic instability of the equilibrium state, as can be seen from the integrated energy budget in Table 6.4.

\subsection{Discussion and conclusions}

Our main goal in this chapter was to perform a linear stability analysis of a thermallyforced 2-layer QG model in order to determine how the circulation transitions from steady to time-dependent when the Reynolds number is increased. We observed an interesting dependence of the type of instabilities that occur on the nondimensional parameter $\Omega$, defined as the ratio of the flow speed $U$ to the the speed of a long internal 
Rossby wave $\beta_{0} R_{d}^{2}$. We verified that the same kind of dependence can be found also in a wind-forced 2-layer QG model, demonstrating that this is not a forcing-dependent feature.

The stability calculations showed that for large values of the $\Omega$ parameter, which can be thought of as a case of a weakly stratified ocean with a small deformation radius, temporal variability that is spatially localized and has inter-monthly periods arises. By spatially localized we mean that the perturbation velocities are confined to the midlatitude jet and recirculation regions, or eventually to the zonal boundary layers next to northern and southern walls for a thermally-forced ocean. An energy analysis showed that in the case of large $\Omega$ the perturbations are generated through baroclinic instabilities of the basis state.

For small values of the $\Omega$ parameter, which can be thought of as a case of more strongly stratified ocean with a larger deformation radius, the circulation becomes instead unstable to perturbations that bear similarity to barotropic Rossby basin modes. Variability that has basin-scale spatial structure, westward phase propagation and monthly periods is generated. For both the wind- and the thermally-forced ocean we determined that the first two destabilizing oscillatory perturbations resemble the $1 \times 2$ and the $2 \times 1$ Rossby basin mode, respectively, but it is not clear if this is always the case. An energy analysis showed that in the case of small $\Omega$ the perturbations are generated through mixed barotropic-baroclinic instabilities of the basic state.

It is not surprising that the type of instability occurring in the model depends on $\Omega$. In particular, larger values of $\Omega$, if all other nondimensional parameters are fixed, signifies a smaller internal deformation radius and thus a flow that is more susceptible to baroclinic type of instabilities, which is essentially what we have observed. The hypothesis that we propose goes a little further and suggests that the type of temporal variability that arises in the model depends actually on the geometry (blocked or closed) of the geostrophic contours $\hat{\phi}$, which on their part depend on the $\Omega$ parameter, since by definition $\hat{\phi}=y+\Omega \phi$ where $\phi$ is the barotropic streamfunction. For configurations with blocked geostrophic contours, spatially localized baroclinic 
variability is preferred, while for configurations with nearly all geostrophic contours blocked barotropic basin mode-like variability appears instead.

For a wind-forced model by using the linear Sverdrup balance one can derive a critical value for $\Omega$ such that when $\Omega>\Omega_{c}$ closed geostrophic contours are formed, while when $\Omega<\Omega_{c}$ all contours are blocked, at least in the linear limit. For a thermallyforced model there is no clear way to predict and distinguish between configurations with closed and blocked geostrophic contours. Following the stationary solution to a low Reynolds number regime, as with the wind-driven model, is not really helpful since for a thermally-forced ocean the barotropic circulation vanishes in the linear limit.

Although all stability calculations presented in this chapter were consistent with our hypothesis, more calculations needs to be done in order establish if the suggested link between the geometry of the geostrophic contours and the type of temporal variability that arises really holds. In particular, it will be useful to trace in the $\Omega-R e$ parameter space the critical curve for onset of a baroclinic type of instability and onset of a basin mode-like type of instability and verify whether the switch between the two is related to the closing of the geostrophic contours. It will be useful also to establish a criterion for a thermally-forced ocean that distinguishes between configurations with closed and blocked geostrophic contours.

If our hypothesis is true then it can have some interesting implications for the real ocean. In general, the geostrophic contours are regarded as a guideline in order to determine where eddy-driven motion in the deep, unventilated layers can be expected. It is possible however that the geometry of the geostrophic contours may also contain information about the type of variability to be expected from a system. If a clear, distinct region of closed geostrophic contours is present, then maybe basin mode-like variability is not to be expected. 


\section{Chapter 7}

\section{Discussion and conclusions}

In this thesis we presented work done on two different problems both involving the stability of large-scale oceanic flows and the importance of non-local effects. We have used the term "non-local effects" to designate phenomena such as radiation of waves or excitation of basin oscillations, generated by a local instability of the flow but that act to expand the influence of the instability to a much broader region than its origin.

\subsection{Radiating instabilities of meridional currents}

The first problem concerned the existence of radiating instabilities for meridional boundary currents. A current is said to be radiatingly unstable if the wavenumbers and frequencies of the perturbations generated by a local instability of the flow are such that they match those of the freely propagating Rossby waves in the ocean interior (McIntyre and Weissman, 1978). In this case, Rossby waves are excited by the local instability that carry energy away from the source (Talley, 1983a; Pierrehumbert, 1984). We used an idealized two-layer quasi-geostrophic configuration with piecewise constant velocity profile in order to examine the linear stability of a meridional boundary current on the $\beta$-plane adjacent to a vast (semi-infinite) motionless ocean interior. We were interested, in particular, in comparing the stability of eastern and western boundary currents, a topic not explored before. 
First of all, our findings made us conclude that meridional currents are generally characterized by radiating instabilities, confirming what was expected from previous studies of currents containing a meridional velocity component (Fantini and Tung, 1987; Kamenkovich and Pedlosky, 1996). This is unlike zonal jets for which special conditions, such as baroclinicity or a westward component of the flow, are needed in order to have radiating instabilities (Talley, 1983a,b). In addition, we found that there are some significant differences in the stability properties that make eastern boundary currents more interesting from a radiation point of view. More specifically, an eastern boundary current has a larger number of radiating modes over a wider range of wavenumbers compared to a western boundary current. The difference between the radiation properties of eastern and western boundary currents is due at its root to the Rossby wave dispersion relation which causes short and long Rossby waves to propagate energy in different zonal directions. Consequently, when unstable, a western boundary current radiates short Rossby waves characterized by a weak eastward group velocity, while an eastern boundary current radiates long Rossby waves characterized by a strong westward group velocity. The latter leads to radiating waves with amplitude envelopes that decay slowly away from the current and makes unstable eastern boundary currents better suited to act as a source of eddy energy for the ocean interior.

Radiation of Rossby waves from the coast of the continents, sometimes referred to as boundary-driven Rossby waves, is not a new concept. Long baroclinic Rossby waves are excited in the ocean interior in response to variable wind that propagate west and reflect into shorter eastward propagating waves (Pedlosky, 1987). Long baroclinic Rossby waves are generated as well on the eastern boundaries of the oceans by poleward-propagating coastal Kelvin waves resulting from wind variability in the equatorial region, providing therefore a tropics-extratropics connection (Jacobs et al., 1994). Our mechanism for wave radiation is different from the previous discussions of boundary-driven Rossby waves in that it is in response to a locally unstable boundary current and not a response to a remote variable wind. Also, our stability analysis 
suggests the radiation from the eastern side of the basins of long barotropic Rossby waves with periods on the order of 100 days or less, while most model studies and altimetry analyses concentrate on the propagation of baroclinic Rossby waves which have longer periods (several months to years), or on barotropic variability but in the western side of the basins (Fu and Qui, 2002; Pierini, 2005). Because of the short periods, radiated barotropic Rossby waves may not be well resolved by satellite altimetry data and thus difficult to find.

A second, unanticipated finding concerned the structure of the waves radiated from the eastern side of a basin. We found that, in general, an unstable eastern boundary current radiates waves that are characterized by a barotropic vertical structure and horizontal wavenumbers such that the zonal wavelength is several times larger than the meridional wavelength. This translates into a velocity field with zonal component much larger than the meridional component, which would make the radiating waves appear more like zonal jets, than localized wave packets or eddies, as they propagate into the ocean interior. The idealized two-layer baroclinic configuration analyzed in this thesis can be thought of as representing a baroclinic current confined to the upper ocean above the thermocline. The majority of the eastern boundary currents are indeed surface intensified and characterized by a baroclinic structure with deep undercurrent flowing in the opposite direction to the surface flow, e.g. Leeuwin current (Smith et al., 1991), California current (Centurioni et al., 2008). The widths of the currents are usually $100 \mathrm{~km}$ or more leading to nondimensional parameters close to those that we used in the stability analysis. We determined that nearly barotropic (over the upper ocean) radiating waves with meridional wavelength on the order of couple of hundred of kilometers and zonal wavelengths several times larger are to be expected. The meridional phase speed of the radiated waves is smaller than that of the current that generates them so eventually they can be arrested by the mean interior gyre flow and lead to time-mean nearly zonal jet-like features.

It is intriguing that stationary quasi-zonal jet-like features (called also striations) have been observed in the eastern parts of the oceans (Maximenko et al., 2005; Cen- 
turioni et al., 2008; Ivanov et al., 2009). Similar quasi-zonal jet-like features can be found also in numerical simulations with high-resolution models, e.g. (Richards et al., 2006). In particular, in the Pacific ocean off the coast of California and Chile, the zonal striations can be identified in variety of time-averaged spatially high-pass filtered data sets such as high-resolution mean dynamic ocean topography, sea surface height, temperature at $100 \mathrm{~m}$ depth, depth of the thermocline as represented by the $12^{\circ} \mathrm{C}$ isotherm (Maximenko et al., 2008). The observed striations have a meridional wavelength of approximatively $400 \mathrm{~km}$ and extend offshore for thousands of kilometers. They retain a coherent vertical structure throughout at least $700 \mathrm{~m}$. The striations are oriented nearly zonally with slight tilt in the direction of the mean interior gyre flow consistent with the idea of a propagating Rossby wave arrested by mean flow.

There is a debate as to the origin of the zonal striations and even questions about their existence, since they may be just an artifact of time-averaging in the presence of propagating mesoscale eddies (Schlax and Chelton, 2008). Our findings raise the possibility that the observed quasi-zonal striations in the eastern parts of the basins may be due to radiating instabilities of eastern boundary currents. Unlike other suggested theories for the generation of zonal jet-like features, e.g. (Kamenkovich et al., 2009), our mechanism does not rely entirely on nonlinear dynamics. Instead it suggests that the observed zonal jet-like features are due to the propagation of Rossby waves arrested by the mean interior flow, i.e. linear dynamics. Because an unstable boundary current is needed to radiate the Rossby waves, the mechanism relies on the presence of a meridional, or nearly meridional, boundary current and thus could be applicable only to the formation of zonal jets in the eastern part of the basins. In order to determine if this is indeed the case however, more works needs to be done, in particular to determine how the radiated waves are affected by the mean interior circulation. All results presented in this thesis were from an idealized configuration using quasi-geostrophic dynamics, constant Rossby wave speed with latitude, zonally uniform meridional boundary current and a motionless interior ocean. This simplified model contains just enough dynamics to show that unstable eastern boundary currents 
possess interesting radiating properties. Simulations with more realistic models will lead to results more directly comparable with observations.

\subsection{Thermally-forced ocean}

The second problem treated in this thesis concerned the circulation in a thermallyforced two-layer QG ocean with nonlinear dynamics. The thermal forcing was introduced in the form of a cross-isopycnal flux, parameterized as relaxation of the interface displacement toward a specified equilibrium profile, similar to the radiative damping forcing commonly used in atmospheric layer QG models (Held, 2000). In the oceanic context, this parameterization can be physically interpreted as a representation of the vertical mixing processes that transfer the surface heat fluxes down the water column to the thermocline. We have focused thus on the large-ocean circulation driven by mixing in the thermocline, while ignoring the wind stress.

The thermal forcing projects only on the baroclinic vertical mode. Consequently, in the linear limit it drives a purely baroclinic circulation (Luyten and Stommel, 1986; Pedlosky and Spall, 2005). However, we showed that taking into consideration the nonlinear advection of vorticity generates a barotropic circulation through the advective coupling to the baroclinic part of the flow. In the steady regime, the barotropic circulation consists of recirculation gyres in the western part of the basin. The barotropic circulation is weaker than the directly thermally-forced baroclinic circulation but it is not negligible. It is strong enough for example to arrest the westward propagation of long baroclinic Rossby waves and create thus regions of closed geostrophic contours isolated from the eastern wall.

The real ocean is driven at the surface by both wind stress and large-scale buoy-

ancy forcing. For the upper ocean, the wind stress is the main driving force, while mixing in the thermocline plays only a secondary role. What our idealized QG model revealed were some properties of the thermally-forced part of the circulation with implications for the real ocean circulation. First of all, because in the presence of 
nonlinear advection a barotropic circulation can be driven by a purely baroclinic forcing, such as the cross-isopycnal flux, for a combined wind- and thermally-forced ocean the wind stress is probably the major but not the only contributor determining the vertically integrated circulation. Especially in the regions of the recirculations, where the nonlinearities are significant, the circulation driven by the cross-isopycnal flux can be important, Second of all, we analyzed the heat budget of our idealized thermally-forced ocean. In the classical paper by Munk (1966) a vertical heat budget for the ocean is assumed, where the vertical velocity $w_{i}$ is entirely diabatic, i.e. a balance between upwelling and downward heat diffusion is assumed. What our simple model shows is that the cross-isopycnal flux represents well the vertical velocity $w_{i}$ only in the linear limit. When nonlinear advection becomes important, nonlinearities and eddies induce a large adiabatic component that dominates the vertical velocity. The adiabatic component can be as large compared to the cross-isopycnal flux as to reverse the sign of the vertical velocity $w_{i}$. Given that in our idealized QG model, the effects of eddies and nonlinearities are not realistically represented, one can expect that in the real ocean the adibatic component of the vertical velocity induced by eddies and nonlinear advection is as important, if not more. This, suggest that a simple one dimensional heat budget is not likely to hold, and advection and eddies should be taken into account.

\subsection{Baroclinic vis basin-scale instabilities}

One of the advantages of using a simple numerical model is that it makes possible to apply techniques from the theory of dynamical systems to understand the variability of the circulation. We thus used a continuation code and linear stability analysis (Dijkstra, 2005) in order to determine the onset of time-dependence in the thermallyforced ocean.

Because the thermally-forced circulation is predominantly baroclinic, and only a weak vertically integrated circulation is produced, we were able to uncover an inter- 
esting, possible link between the type of perturbations that lead to time-dependence of the circulation and the geometry of the geostrophic contours. Furthermore, we confirmed that the same possible connection between temporal variability and geostrophic contours exists for wind-driven double-gyre flows as well. The geostrophic contours $\hat{\phi}$ are defined as $\hat{\phi}=y+\Omega \phi$, where $\phi$ is the barotropic streamfunction and $\Omega=U / \beta_{0} R_{d}^{2}$, a nondimensional parameter equal to the ratio of the flow speed to the speed of long internal Rossby waves. They represent the curves along which information about the blocking action of the eastern wall is propagated westward in the basin by the long internal Rossby waves (Rhines and Young, 1982). For a wind-forced ocean they coincide with the deep layer streamlines, while this is not true for a thermally-forced ocean.

We found that for large values of $\Omega$, when a region of closed geostrophic contours tends to form, the circulation becomes unstable to baroclinic type of instabilities with inter-monthly timescales and is spatially confined to the midlatitude zonal jet or the zonal boundary layers next to the southern and northern walls. For small values of $\Omega$, when the geostrophic contours (especially in the low Reynolds number limit) are mostly blocked, variability resembling barotropic Rossby basin modes arises instead. It is characterized by basin-scale spatial structure, shorter monthly periods and is due to a mixed barotropic-baroclinic variability of the circulation.

More calculations need to be done in order to verify our hypothesis that the type of temporal variability that arises in a system is related to the geometry of its geostrophic contours. If true, this can have some interesting implications. For example, if a large region of homogenized potential vorticity is observed in the deep parts of a basin, indicative of a region of closed geostrophic contours, then maybe localized, baroclinictype of variability should be expected for the circulation instead of basin modelike variability. Climatological maps of the large-scale potential vorticity field along isopycnals for the global ocean show that regions of homogenized potential vorticity are present in the deep waters of the North Pacific and, possibly, in the bottom waters of the western North Atlantic and North Pacific (O’Dwyer and Williams, 1997). In 
both these basins, localized baroclinic instabilities associated with meandering and shifts of the free mid-latitude jet and the associated recirculations are the dominant mode of variability (Dijkstra, 2005).

\subsection{Temporal variability dominated by barotropic basin modes}

Finally, we examined the thermally-forced circulation in the strongly nonlinear regime. It was determined that in this case the system's variability is dominated by barotropic Rossby basin modes, unless they are damped by bottom friction. We treated this regime of circulation as an example of a system whose temporal variability is described to a large degree by barotropic basin modes. In other words, we suggest that the results from this regime are likely to be applicable in a more general context where the type of forcing that drives the circulation (wind or thermal) is not important, but what matters is rather the excitation of barotropic basin-scale oscillations. We showed that the presence of strong basin mode variability affects significantly the circulation - it rectifies a multi-gyre time-mean barotropic circulation, it forces baroclinic variability on similar timescales to those of the dominant basin modes and it interferes with the eddy-driven recirculations.

Satellite altimetry is useful for the detection of the barotropic variability of the oceans. For a basin the size of the Atlantic or the Pacific Ocean however the periods of the barotropic Rossby basin modes are too short (O(15days) or less) to be resolved by the altimetry data - the Topex/Poseidon has a 20-day Nyquist period, (Le Traon and Morrow, 2000). However, barotropic oscillations consistent with Rossby basin modes have been observed in basins semi-enclosed by bathymetry(Warren et al., 2002; Fu et al., 2001; Weijer et al., 2007a) and marginal seas (Stanev and Rachev, 1999). These are all examples of smaller in size basins compared to the full Atlantic or Pacific Ocean basins. Because the frequency of the barotropic basin modes increases with the basin size (Pedlosky, 1987), the period of the barotropic Rossby modes in 
these smaller basins is on the order of 25-50 days. This makes them more suitable for detecting in altimetry data. Marginal seas and semi-enclosed basins represent thus examples of places in the world ocean where the connection between mean circulation, mesoscale variability and high-frequency basin modes is worth exploring further with more appropriate regional models.

One piece missing from the analysis presented in this thesis concerning the basin mode-dominated variability, but that it would be useful to provide, is to examine the Lagrangian dynamics of the circulation. Because the multi-gyre time-mean circulation present in the strongly nonlinear regime results from a wave phenomena (the basin modes), it is not clear whether the fluid particles will follow the time-mean streamlines. 


\section{Appendix A}

\section{Method of solution for the}

\section{radiating instability problem}

\section{A.1 The barotropic case}

The linear stability equation (2.3) to be solved is a constant coefficient ODE since the basic state velocity is constant in the two regions, boundary current $|x|<x_{0}$ and far field $|x|>x_{0}$. The solution in the boundary current region, where $V$ is a non-zero constant, is

$$
\phi^{i n}(x)=\sum_{j=1}^{2} B_{j} e^{i k_{j} x}
$$

where the zonal wavenumbers $k_{j}$ are the two roots of the 2 nd order polynomial

$$
k^{2}(V-c)-\frac{\beta}{m} k+m^{2}(V-c)=0 .
$$

The solution in the far field region is a particular case with $V=0$ of the boundary current solution. The two roots of the 2 nd order polynomial in this case are

$$
k_{b t}^{ \pm}=\frac{\beta}{2 c m}\left[-1 \pm \sqrt{1-\frac{4 c^{2} m^{4}}{\beta^{2}}}\right]
$$


Only one of the zonal wavenumbers $k_{b t}^{ \pm}$is physically consistent for the far field solution. The minus sign corresponds to barotropic Rossby waves that have positive group velocity - this is the choice for a western boundary current. The plus sign corresponds to barotropic Rossby waves that have negative group velocity - this is the choice for an eastern boundary. Therefore, the solution in the far field region, for the western and eastern boundary current respectively, is

$$
\phi^{\text {out }}(x)=B_{3} e^{i k_{b t}^{\mp}\left(x \mp x_{0}\right)} .
$$

For given parameter $\beta$ and meridional wavenumber $m$, the constants $B_{j}$ and the eigenvalue(s) $c$, which appear in the solutions (A.1) and (A.2) through the expressions for the zonal wavenumbers, are found by imposing the no-normal flow condition at the wall and the jump conditions (2.5) on the side with discontinuous velocity. These conditions translate into the following set of equations, for the western and eastern case respectively

$$
\begin{aligned}
\text { at } x=\mp x_{0}: & \phi^{\text {in }}=0, \\
\text { at } x= \pm x_{0}: & \frac{\phi^{\text {in }}}{V-c}=\frac{\phi^{\text {out }}}{-c}, \\
\text { at } x= \pm x_{0}: & (V-c) \frac{d \phi^{\text {in }}}{d x}+\frac{\beta}{i m} \phi^{\text {in }}=-c \frac{d \phi^{\text {out }}}{d x}+\frac{\beta}{i m} \phi^{\text {out }} .
\end{aligned}
$$

It is straightforward to see that, after using the expressions (A.1) and (A.2), the equations above lead to a homogeneous 3 by 3 system for the unknown constants $\left\{B_{j}\right\}_{j=1}^{3}$. The eigenvalues $c$ are those values for $c$ that make the determinant of the homogeneous system zero so that there is a non-trivial solution for the constants $B_{j}$. Once the eigenvalue(s) $c$ are found, if there are such, the solution in all regions can be reconstructed. 


\section{A.2 The baroclinic case}

The linear stability problem (2.12) is solved following the same procedure except that now we are dealing with 2 layers. The solution in the boundary current region, where $V_{n}$ are non-zero constants, is

$$
\begin{aligned}
\phi_{1}^{i n}(x) & =\sum_{j=1}^{4} B_{j} e^{i k_{j} x}, \\
\phi_{2}^{i n}(x) & =\sum_{j=1}^{4} B_{j} \Gamma_{j} e^{i k_{j} x},
\end{aligned}
$$

where

$$
\Gamma_{j}=\frac{k_{j}^{2}}{F_{1}}+\frac{\beta k_{j}}{m\left(c-V_{1}\right) F_{1}}+\frac{m^{2}}{F_{1}}+\frac{c-V_{2}}{c-V_{1}} .
$$

and the zonal wavenumbers $k_{j}$ are the four roots of the 4 th order polynomial

$$
\alpha_{4} k^{4}+\alpha_{3} k^{3}+\alpha_{2} k^{2}+\alpha_{1} k+\alpha_{0}=0
$$

with

$$
\begin{aligned}
\alpha_{4} & =\left(c-V_{1}\right)\left(c-V_{2}\right), \\
\alpha_{3} & =\left(2 c-V_{1}-V_{2}\right), \\
\alpha_{2} & =\left(\frac{\beta}{m}\right)^{2}+\left(2 m^{2}+F\right) \alpha_{4}+\left(V_{1}-V_{2}\right) \gamma, \\
\alpha_{1} & =\frac{\beta}{m}\left(m^{2} \alpha_{3}+\gamma\right), \\
\alpha_{0} & =m^{2}\left[\left(m^{2}+F\right) \alpha_{4}+\left(V_{1}-V_{2}\right) \gamma\right], \\
\gamma & =F_{1}\left(c-V_{2}\right)-F_{2}\left(c-V_{1}\right) .
\end{aligned}
$$

In the far field region, where $V_{n}=0$, the 4 th order polynomial reduces to the 
barotropic and baroclinic Rossby dispersion relations so that the four roots $k_{j}$ become

$$
\begin{aligned}
& k_{b t}^{ \pm}=\frac{\beta}{2 c m}\left[-1 \pm \sqrt{1-\frac{4 c^{2} m^{4}}{\beta^{2}}}\right] \\
& k_{b c}^{ \pm}=\frac{\beta}{2 c m}\left[-1 \pm \sqrt{1-\frac{4 c^{2} m^{2}\left(m^{2}+F\right)}{\beta^{2}}}\right]
\end{aligned}
$$

Once more, only one of the signs in the expressions above corresponds to barotropic and baroclinic Rossby wave with zonal group velocity in the right direction. Thus, the solution in the far field region, for the western and eastern case respectively, is

$$
\begin{aligned}
\phi_{1}^{\text {out }}(x) & =\frac{1}{2} B_{5} e^{i k_{b t}^{\mp}\left(x \mp x_{0}\right)}+\frac{F_{1}}{F} B_{6} e^{i k_{b c}^{\mp}\left(x \mp x_{0}\right)}, \\
\phi_{2}^{\text {out }}(x) & =\frac{1}{2} B_{5} e^{i k_{b t}^{\mp}\left(x \mp x_{0}\right)}-\frac{F_{2}}{F} B_{6} e^{i k_{b c}^{\mp}\left(x \mp x_{0}\right)} .
\end{aligned}
$$

Applying the no-normal flow condition at the wall and the jump conditions (2.5) for each layer translates into the following set of equations

$$
\begin{aligned}
\text { at } x=\mp x_{0}: & \phi_{n}^{\text {in }}=0, n=1,2, \\
\text { at } x= \pm x_{0}: & \frac{\phi_{n}^{\text {in }}}{V_{n}-c}=\frac{\phi_{n}^{\text {out }}}{-c}, n=1,2, \\
\text { at } x= \pm x_{0}: & \left(V_{n}-c\right) \frac{d \phi_{n}^{\text {in }}}{d x}+\frac{\beta}{i m} \phi_{n}^{\text {in }}=-c \frac{d \phi_{n}^{\text {out }}}{d x}+\frac{\beta}{i m} \phi_{n}^{\text {out }}, n=1,2 .(\text {. }
\end{aligned}
$$

This leads, after using the expressions (A.6) and (A.7), to a homogeneous 6 by 6 system for the unknown constants $\left\{B_{j}\right\}_{j=1}^{6}$. The eigenvalues $c$ are those values for $c$ that make the determinant of the homogeneous system zero so that there is a non-trivial solution for the constants $B_{j}$. Once the eigenvalue(s) $c$ are found, if there are such, the solution in all regions and layers can be reconstructed. 


\section{Appendix B}

\section{Friction scales}

Three different types of dissipation are included in the 2-layer QG model: lateral diffusion of relative vorticity, bottom drag and interface damping. A simple analysis is presented here in order to determine the spatial scales damped selectively by each type of dissipation.

Let a perturbation flow $\tilde{\psi}_{n}$ is added to the circulation driven by the applied forcing (wind or thermal) as described by Eq.(3.9) and (3.10). If all contributions from the nonlinear advective terms are neglected, then the evolution of the perturbation flow is governed by the linearized unforced potential vorticity equations

$$
\begin{aligned}
\frac{\partial}{\partial t}\left(\nabla^{2} \tilde{\psi}_{1}-F_{1}\left(\tilde{\psi}_{1}-\tilde{\psi}_{2}\right)\right) & =\nu \nabla^{2}\left(\nabla^{2} \tilde{\psi}_{1}\right)+\frac{F_{1}}{\gamma}\left(\tilde{\psi}_{1}-\tilde{\psi}_{2}\right) \\
\frac{\partial}{\partial t}\left(\nabla^{2} \tilde{\psi}_{2}+F_{2}\left(\tilde{\psi}_{1}-\tilde{\psi}_{2}\right)\right) & =\nu \nabla^{2}\left(\nabla^{2} \tilde{\psi}_{2}\right)-\frac{F_{2}}{\gamma}\left(\tilde{\psi}_{1}-\tilde{\psi}_{2}\right)-r \nabla^{2} \tilde{\psi}_{2}
\end{aligned}
$$

The solution of this system of equations is of the form

$$
\tilde{\psi}_{n}=A_{n} e^{i(k x+l y)} e^{\sigma t}
$$

where the wavenumbers $k$ and $l$ define the spatial scale $\Lambda=\left(k^{2}+l^{2}\right)^{-1 / 2}$ of the perturbation flow, while $\sigma$ is its decay rate. Replacing Eq.(B.3) into the system of equations leads to the following expression for the decay rate, relating $\sigma$ to the 
different frictional coefficients

$$
\sigma=-\nu \frac{R_{d}^{2}}{\Lambda^{2}\left(\Lambda^{2}+R_{d}^{2}\right)}-r \frac{\frac{H_{2}}{H} \Lambda^{2}+R_{d}^{2}}{\Lambda^{2}+R_{d}^{2}}-\frac{1}{\gamma} \frac{\Lambda^{2}}{\Lambda^{2}+R_{d}^{2}},
$$

where $\nu$ is the eddy viscosity, $r$ the bottom drag, and $\gamma$ the interface relaxation timescale. In writing the expression above we have used that $F_{1}+F_{2}=1 / R_{d}^{2}$.

The lateral diffusions damps selectively the smallest scales present in the model, while the flow on scales much larger than the deformation is essentially unaffected. The bottom drag is nearly scale insensitive - flow on spatial scales much larger than the deformation radius are damped slightly less (by a factor of $H_{2} / H$ ) than the flow on spatial scales much smaller than the deformation radius. Finally, the interfacial relaxation damps selectively the flow on large spatial scales while the smallest scales are left essentially unaffected. 


\section{Appendix C}

\section{Hilbert empirical orthogonal functions analysis}

This is a brief overview of the basic principles and terminology of the Hilbert empirical orthogonal functions (HEOF) statistical analysis, following closely Terradas et al. (2004).

Let $X\left(x_{j}, t\right)$ be an anomaly scalar field, i.e with the time-mean subtracted, where $x_{j}$ is a grid position and $t$, the time. The core of the HEOF analysis consists in finding the eigenvalues and eigenvectors of the covariance matrix $C$

$$
C_{i j}=\left\langle U^{*}\left(x_{i}, t\right) U\left(x_{j}, t\right)\right\rangle_{t}
$$

where the asterisk denotes complex conjugation, the brackets time averaging and the complex field $U\left(x_{j}, t\right)$ is defined as having real and imaginary parts equal to the scalar data field and its Hilbert transform, respectively

$$
U\left(x_{j}, t\right)=X\left(x_{j}, t\right)+i \hat{X}\left(x_{j}, t\right)
$$

By definition, the Hilbert transform of a data series has Fourier spectral components with the same amplitude as the original scalar field, while the phase is advanced 
by $\pi / 2$. If at a given position $x_{j}$, the spectral decomposition of $X\left(x_{j}, t\right)$ is

$$
X\left(x_{j}, t\right)=\sum_{\omega} a_{j}(\omega) \cos (\omega t)+b_{j}(\omega) \sin (\omega t)
$$

then its Hilbert transform $\hat{X}\left(x_{j}, t\right)$ is defined as

$$
\hat{X}\left(x_{j}, t\right)=\sum_{\omega} b_{j}(\omega) \cos (\omega t)-a_{j}(\omega) \sin (\omega t)
$$

Were the data series described by a single frequency oscillation, the Hilbert transform would be proportional simply to its time derivative. In the general case, the Hilbert transform provides information, locally in the frequency domain, about the time rate of change of the scalar field. In the standard EOF analysis, the covariance matrix $C_{i j}$ is computed using only the scalar field $X\left(x_{j}, t\right)$. It is by retaining information about the phase of the scalar field, that the HEOF analysis is able to detect propagating oscillations.

The covariance matrix $C$ defined by Eq.(C.1) is Hermitian by construction and possesses thus $m$ real and nonnegative eigenvalues $\lambda_{n}$, with corresponding orthogonal complex eigenvectors $E_{n}\left(x_{j}\right), n=1,2, \ldots m$. By definition, the $n$th HEOF mode or statistical mode consists of a spatial part, the eigenvector $E_{n}\left(x_{j}\right)$, and a temporal part, the complex principal component $A_{n}(t)$, obtained by projecting the complex data field $U\left(x_{j}, t\right)$ on the eigenvector $E_{n}\left(x_{j}\right)$ and summing over all locations

$$
A_{n}(t)=\sum_{j} U\left(x_{j}, t\right) E_{n}\left(x_{j}\right)
$$

The original complex field $U\left(x_{j}, t\right)$ can be fully reconstructed using the empirically found statistical modes

$$
U\left(x_{j}, t\right)=\sum_{n=1}^{m} E_{n}^{*}\left(x_{j}\right) A_{n}(t) .
$$

Each statistical mode product $E_{n}^{*}\left(x_{j}\right) A_{n}(t)$ represents an oscillatory component present in the field. The eigenvalues provide a simple way to quantify how much each 
mode contributes to the total field variance, since

$$
\frac{\lambda_{n}}{\sum_{k=1}^{m} \lambda_{k}}=\frac{\lambda_{n}}{\operatorname{var}(X)}
$$

given that $\sum_{k=1}^{m} \lambda_{k}=\operatorname{Trace}(C)=\operatorname{var}(X)$. Normally, the eigenvalues are sorted out in descending order so that the rank $1 \mathrm{HEOF}$ has the largest eigenvalues, i.e it explains the largest portion of the field variance, the rank $2 \mathrm{HEOF}$ is the 2 nd most important, and so on.

It is convenient to rewrite the decomposition of the data field $U\left(x_{j}, t\right)$ onto the complex basis functions $E_{n}\left(x_{j}\right)$ with complex coefficients $A_{n}(t)$ given by Eq.(C.6), using instead real-valued functions

$$
U\left(x_{j}, t\right)=\sum_{n=1}^{m} S_{n}\left(x_{j}\right) e^{-i \theta_{n}\left(x_{j}\right)} R_{n}(t) e^{i \varphi_{n}(t)}
$$

or, for the original data field $X\left(x_{j}, t\right)$ which is simply the real part of $U\left(x_{j}, t\right)$,

$$
X\left(x_{j}, t\right)=\sum_{n=1}^{m} S_{n}\left(x_{j}\right) R_{n}(t) \cos \left[\varphi_{n}(t)-\theta_{n}\left(x_{j}\right)\right]
$$

The four real-valued functions $S_{n}\left(x_{j}\right), \theta_{n}\left(x_{j}\right), R_{n}(t), \varphi_{n}(t)$ describe different aspects of the oscillatory components present in the data field and are defined in a straightforward way in terms of the HEOFs and principal components.

1. Spatial amplitude function $S_{n}\left(x_{j}\right)$,

$$
S_{n}\left(x_{j}\right)=\left[E_{n}\left(x_{j}\right) E_{n}^{*}\left(x_{j}\right)\right]^{\frac{1}{2}}
$$

The spatial amplitude may be interpreted in the same way as the spatial pattern in standard EOF analysis. It represents a measure of the spatial distribution of variability in the data field $X$ associated with a given statistical mode. 
2. Spatial phase function $\theta_{n}\left(x_{j}\right)$,

$$
\theta_{n}\left(x_{j}\right)=\arctan \left[\frac{\operatorname{Im}\left(E_{n}\left(x_{j}\right)\right)}{\operatorname{Re}\left(E_{n}\left(x_{j}\right)\right)}\right]
$$

The spatial phase varies continuously between $-\pi$ and $\pi$. It represents a measure of the relative phase difference for a given statistical mode among the various locations where $X$ is defined. Locally, its derivative is equal to the phase speed of the propagating oscillation.

3. Temporal amplitude function $R_{n}(t)$,

$$
R_{n}(t)=\left[A_{n}(t) A_{n}^{*}(t)\right]^{\frac{1}{2}}
$$

The temporal amplitude may be interpreted in the same way as the principal component in standard EOF analysis. It represents a measure of the temporal variability in the magnitude associated with a given statistical mode.

4. Temporal phase function $\varphi_{n}(t)$,

$$
\varphi_{n}(t)=\arctan \left[\frac{\operatorname{Im}\left(A_{n}(t)\right)}{\operatorname{Re}\left(A_{n}(t)\right)}\right] .
$$

The temporal phase varies continuously between $-\pi$ and $\pi$. It represents a measure of the temporal variation of the phase of a given statistical mode associated with periodicities in the data field $X$. Locally, its derivative is equal to the frequency of the propagating oscillation.

Often, the amplitude and phase functions are more useful for the interpretation and understanding of the statistical modes than looking directly at the real and imaginary part of the HEOFs and their principal components. 


\section{Appendix D}

\section{Perturbation energy equations}

\section{D.1 Derivation of the perturbation energy equa- tions}

One way to write the QG potential vorticity equations for a 2-layer ocean, convenient when deriving the energy budget, is to separate the equations governing the evolution of the relative vorticity by density layers from those governing the evolution of the interface displacement

$$
\begin{aligned}
-\frac{\partial}{\partial t}\left(\nabla^{2} \psi_{1}\right) & =J\left(\psi_{1}, \nabla^{2} \psi_{1}+\beta y\right)-U_{w} F_{w}(y)+d_{2} F w_{i}-\frac{1}{R e} \nabla^{4} \psi_{1} \\
\frac{\partial}{\partial t}\left(\psi_{1}-\psi_{2}\right) & =-J\left(\psi_{1}, \psi_{1}-\psi_{2}\right)-w_{i}+w_{*} \\
-\frac{\partial}{\partial t}\left(\nabla^{2} \psi_{2}\right) & =J\left(\psi_{2}, \nabla^{2} \psi_{2}+\beta y\right)-d_{1} F w_{i}-\frac{1}{R e} \nabla^{4} \psi_{2}, \\
\frac{\partial}{\partial t}\left(\psi_{1}-\psi_{2}\right)= & -J\left(\psi_{2}, \psi_{1}-\psi_{2}\right)-w_{i}+w_{*}, \\
& w_{*}=-\frac{\beta}{F \delta_{T}}\left(\psi_{1}-\psi_{2}+U_{T} F_{T}(y)\right) .
\end{aligned}
$$

The second and forth equations above are mathematically identical. Physically, one can be thought as describing the evolution of the upward interface displacement, while 
the other as describing the evolution of the downward interface displacement. When written in this form, there are actually three unknown in the QG potential vorticity equations - the streamfunction for the two density layers $\psi_{1}$ and $\psi_{2}$, and the vertical velocity at the interface $w_{i}$.

Let decompose the flow into a stationary solution given by $\left\{\Psi_{1}, \Psi_{2}, W_{i}\right\}$ and a perturbation flow $\left\{\psi_{1}^{\prime}, \psi_{2}^{\prime}, w_{i}^{\prime}\right\}$

$$
\psi_{1}=\Psi_{1}+\psi_{1}^{\prime}(x, y, t), \quad \psi_{2}=\Psi_{2}+\psi_{2}^{\prime}(x, y, t), \quad w_{i}=W_{i}+w_{i}^{\prime}(x, y, t) .
$$

Assuming that the amplitude of the perturbation is small, its evolution is governed by the linearized perturbation QG potential vorticity equations

$$
\begin{gathered}
-\frac{\partial}{\partial t}\left(\nabla^{2} \psi_{1}^{\prime}\right)=J\left(\Psi_{1}, \nabla^{2} \psi_{1}^{\prime}\right)+J\left(\psi_{1}^{\prime}, \nabla^{2} \Psi_{1}+\beta y\right)+d_{2} F w_{i}^{\prime}-\frac{1}{R e} \nabla^{4} \psi_{1}^{\prime} \\
\frac{\partial}{\partial t}\left(\psi_{1}^{\prime}-\psi_{2}^{\prime}\right)=-J\left(\Psi_{1}, \psi_{1}^{\prime}-\psi_{2}^{\prime}\right)-J\left(\psi_{1}^{\prime}, \Psi_{1}-\Psi_{2}\right)-w_{i}^{\prime}-\frac{\beta}{F \delta_{T}}\left(\psi_{1}^{\prime}-\psi_{2}^{\prime}\right) \\
-\frac{\partial}{\partial t}\left(\nabla^{2} \psi_{2}^{\prime}\right)=J\left(\Psi_{2}, \nabla^{2} \psi_{2}^{\prime}\right)+J\left(\psi_{2}^{\prime}, \nabla^{2} \Psi_{2}+\beta y\right)-d_{1} F w_{i}^{\prime}-\frac{1}{R e} \nabla^{4} \psi_{2}^{\prime} \\
\frac{\partial}{\partial t}\left(\psi_{1}^{\prime}-\psi_{2}^{\prime}\right)=-J\left(\Psi_{2}, \psi_{1}^{\prime}-\psi_{2}^{\prime}\right)-J\left(\psi_{2}^{\prime}, \Psi_{1}-\Psi_{2}\right)-w_{i}^{\prime}-\frac{\beta}{F \delta_{T}}\left(\psi_{1}^{\prime}-\psi_{2}^{\prime}\right)
\end{gathered}
$$

Multiplying the perturbation relative vorticity equations by the respective perturbation streamfunction weighted by the layer depth $d_{n} \psi_{n}^{\prime}$, leads to an equation for the perturbation kinetic energy by density layer. Multiplying the perturbation interface displacement equations by $d_{1} d_{2} F\left(\psi_{1}^{\prime}-\psi_{2}^{\prime}\right)$ and adding them together, leads to an equation for the perturbation potential energy.

The following perturbation energy budget is obtained when the perturbation energy equations are in addition integrated over the basin area and averaged over some 
time interval

$$
\begin{aligned}
& \frac{\partial}{\partial t} \iint \frac{d_{n}}{2} \overline{\left(\nabla \psi_{n}^{\prime}\right)^{2}}=\underbrace{\iint d_{n} \overline{\psi_{n}^{\prime} J\left(\Psi_{n}, \nabla^{2} \psi_{n}^{\prime}\right)}}_{B T_{n}}+\iint d_{n} \overline{\psi_{n}^{\prime} J\left(\psi_{n}^{\prime}, \nabla^{2} \Psi_{n}+\beta y\right)} \\
& -\underbrace{\iint(-1)^{n} d_{1} d_{2} F \overline{\left(\psi_{n}^{\prime} w_{i}^{\prime}\right)}}_{\text {transfer } K E \rightarrow P E}-\underbrace{\iint \frac{d_{n}}{R e} \overline{\psi_{n}^{\prime} \nabla^{4} \psi_{n}^{\prime}}}_{\text {dissipation }} \\
& \frac{\partial}{\partial t} \iint \frac{d_{1} d_{2} F}{2} \overline{\left(\psi_{1}^{\prime}-\psi_{2}^{\prime}\right)^{2}}=\iint \frac{d_{1} d_{2} F}{2} \overline{\left(\psi_{1}^{\prime}-\psi_{2}^{\prime}\right)\left[J\left(\Psi_{1}, \psi_{1}^{\prime}-\psi_{2}^{\prime}\right)+J\left(\Psi_{2}, \psi_{1}^{\prime}-\psi_{2}^{\prime}\right)\right]} \\
& +\underbrace{\iint \frac{d_{1} d_{2} F}{2} \overline{\left(\psi_{1}^{\prime}-\psi_{2}^{\prime}\right)\left[J\left(\psi_{1}^{\prime}, \Psi_{1}-\Psi_{2}\right)+J\left(\psi_{2}^{\prime}, \Psi_{1}-\Psi_{2}\right)\right]}}_{B C} \\
& -\underbrace{\iint d_{1} d_{2} F \overline{\left(\psi_{1}^{\prime}-\psi_{2}^{\prime}\right) w_{i}^{\prime}}}_{\text {transfer } P E \rightarrow K E}-\underbrace{\iint d_{1} d_{2} \frac{\beta}{\delta_{T}} \overline{\left(\psi_{1}^{\prime}-\psi_{2}^{\prime}\right)^{2}}}_{\text {dissipation }}
\end{aligned}
$$

The two energy conversion terms $B T_{n}$ and $B C$ represent the two pathways through which the energy of the equilibrium state can be transfered to the perturbation flow and lead to growth. There are in addition energy exchange terms where perturbation kinetic and potential energy is transformed. The perturbation kinetic energy is dissipated only through the eddy viscosity, while for the potential energy there is also damping because of the thermal relaxation. Finally, all integrals that are without notation can be shown to vanish in the case of no-slip boundary conditions and thus do not contribute to the energy budget.

The total perturbation energy is equal to the sum of the kinetic energy for the two layers and the potential energy for the perturbation flow

$$
E=\frac{1}{2} \iint d_{1} \overline{\left(\nabla \psi_{1}^{\prime}\right)^{2}}+d_{2} \overline{\left(\nabla \psi_{2}^{\prime}\right)^{2}}+d_{1} d_{2} \overline{F\left(\psi_{1}^{\prime}-\psi_{2}^{\prime}\right)^{2}}
$$

An equation for its evolution can be obtained by summing the three integrated energy equations. This leads to the cancelation of the energy transfer terms. Thus, 
the following net perturbation energy budget is left

$$
\frac{\partial E}{\partial t}=B T_{1}+B T_{2}+B C-\iint \sum_{n=1}^{2} \frac{d_{n}}{R e} \overline{\psi_{n}^{\prime} \nabla^{4} \psi_{n}^{\prime}}-\iint d_{1} d_{2} \frac{\beta}{\delta_{T}} \overline{\left(\psi_{1}^{\prime}-\psi_{2}^{\prime}\right)^{2}}
$$

The total perturbation energy can grow due to the barotropic and/or baroclinic energy conversion terms and is dissipated by eddy viscosity and by the interface relaxation, if active.

It can be shown using the divergence theorem, that in the case of no-slip boundary conditions the barotropic and baroclinic energy conversion terms are proportional to respectively the horizontal and the vertical equilibrium velocity field gradients

$$
\begin{aligned}
& B T_{n}=\iint d_{n} \overline{\psi_{n}^{\prime} J\left(\Psi_{n}, \nabla^{2} \psi_{n}^{\prime}\right)}=\iint d_{n} \frac{\overline{\partial \psi_{n}^{\prime}}}{\partial x_{i}} \frac{\partial \psi_{n}^{\prime}}{\partial x_{j}} \frac{\partial U_{n, j}}{\partial x_{i}} \\
& =\iint d_{n}\left[\overline{\left(\frac{\partial \psi_{n}^{\prime}}{\partial x}\right)^{2}} \frac{\partial U_{n}}{\partial x}+\overline{\frac{\partial \psi_{n}^{\prime}}{\partial x} \frac{\partial \psi_{n}^{\prime}}{\partial y}}\left(\frac{\partial V_{n}}{\partial x}+\frac{\partial U_{n}}{\partial y}\right)+\overline{\left(\frac{\partial \psi_{n}^{\prime}}{\partial y}\right)^{2}} \frac{\partial V_{n}}{\partial y}\right], \\
& B C=\iint \frac{d_{1} d_{2} F}{2} \overline{\left(\psi_{1}^{\prime}-\psi_{2}^{\prime}\right)\left[J\left(\psi_{1}^{\prime}, \Psi_{1}-\Psi_{2}\right)+J\left(\psi_{2}^{\prime}, \Psi_{1}-\Psi_{2}\right)\right]} \\
& =\iint d_{1} d_{2} F \overline{\psi_{1}^{\prime} J\left(\Psi_{1}-\Psi_{2}, \psi_{2}^{\prime}\right)} \\
& =\iint d_{1} d_{2} F\left[\overline{\psi_{1}^{\prime} \frac{\partial \psi_{2}^{\prime}}{\partial x}}\left(U_{1}-U_{2}\right)+\overline{\psi_{1}^{\prime} \frac{\partial \psi_{2}^{\prime}}{\partial y}}\left(V_{1}-V_{2}\right)\right] \text {, }
\end{aligned}
$$

where $U_{n}=-\partial \Psi_{n} / \partial y$ and $V_{n}=\partial \Psi_{n} / \partial x$ denote the equilibrium state velocity field.

\section{D.2 Normal mode analysis}

When examining the stability of the equilibrium state $\left\{\Psi_{1}, \Psi_{2}\right\}$, the perturbation flow is taken of the form

$$
\psi_{n}^{\prime}(x, y, t)=\operatorname{Real}\left(\hat{\psi}_{n}(x, y) e^{c t}\right)=\frac{e^{c_{r} t}}{2}\left(\hat{\psi}_{n} e^{i c_{i} t}+\hat{\psi}_{n}^{*} e^{-i c_{i} t}\right)
$$


where $\hat{\psi}_{n}$ is a complex amplitude describing the spatial structure of the perturbation, while $\sigma$ is the growth rate describing its temporal structure.

In this case, after some algebraic manipulations it can be shown that the total perturbation energy averaged over an oscillation period becomes

$$
E=\frac{1}{2} \iint d_{1}\left|\nabla \hat{\psi}_{1}\right|^{2}+d_{2}\left|\nabla \hat{\psi}_{2}\right|^{2}+d_{1} d_{2} F\left|\hat{\psi}_{1}-\hat{\psi}_{2}\right|^{2}
$$

Essentially the same energy budget holds

$$
\sigma_{r} E=\operatorname{Real}\left(B T_{1}+B T_{2}+B C-\iint \sum_{n=1}^{2} \frac{d_{n}}{R e} \hat{\psi}_{n}^{*} \nabla^{4} \hat{\psi}_{n}-\iint d_{1} d_{2} \frac{\beta}{\delta_{T}}\left|\hat{\psi}_{1}-\hat{\psi}_{2}\right|^{2}\right)
$$

where the barotropic and baroclinic conversion terms become

$$
\begin{aligned}
B T_{n} & =\iint d_{n} \hat{\psi}_{n}^{*} J\left(\Psi_{n}, \nabla^{2} \hat{\psi}_{n}\right) \\
& =\iint d_{n}\left[\left|\frac{\partial \hat{\psi}_{n}}{\partial x}\right|^{2} \frac{\partial U_{n}}{\partial x}+\frac{\partial \hat{\psi}_{n}^{*}}{\partial x} \frac{\partial \hat{\psi}_{n}}{\partial y}\left(\frac{\partial V_{n}}{\partial x}+\frac{\partial U_{n}}{\partial y}\right)+\left|\frac{\partial \hat{\psi}_{n}}{\partial y}\right|^{2} \frac{\partial V_{n}}{\partial y}\right] \\
B C & =\iint d_{1} d_{2} F \hat{\psi}_{1}^{*} J\left(\Psi_{1}-\Psi_{2}, \hat{\psi}_{2}\right) \\
& =\iint d_{1} d_{2} F\left[\hat{\psi}_{1}^{*} \frac{\partial \hat{\psi}_{2}}{\partial x}\left(U_{1}-U_{2}\right)+\hat{\psi}_{1}^{*} \frac{\partial \hat{\psi}_{2}}{\partial y}\left(V_{1}-V_{2}\right)\right]
\end{aligned}
$$

The star in all equations above stands for complex conjugation. 


\section{Bibliography}

Arakawa, A., 1997: Computational design for long-term numerical integration of the equations of fluid motion: Two-dimensional incompressible flow. part 1. J. Comput. Phys., 135, 103-114, article no. CP975697.

Berloff, P. and J. C. McWilliams, 1999a: Large-scale, low-frequency variability in wind-driven ocean gyres. J. Phys. Oceanogr., 29, 1925-1949.

Berloff, P. and J. C. McWilliams, 1999b: Quasi-geostrophic dynamics of the western boundary current. J. Phys. Oceanogr., 29, 2607-2634.

Berloff, P. and S. P. Meacham, 1998: The dynamics of a simple baroclinic model of the wind-driven circulation. J. Phys. Oceanogr., 28, 361-388.

Centurioni, L. R., J. C. Ohlmann, and P. P. Niiler, 2008: Permanent meanders in the California current system. J. Phys. Oceanogr., 38, 1690-1710.

Cessi, P., G. Ierley, and W. Young, 1987: A model of inertial recirculation driven by potential vorticity dynamics. J. Phys. Oceanogr., 17, 1640-1652.

Davey, M. K., 1983: A two-level model of a thermally forced ocean basin. J. Phys. Oceanogr., 13, 169-190.

Dijkstra, H. A., 2005: Nonlinear physical oceanography. A dynamical systems approach to the large scale ocean circulation and El Niño. 2d ed., Springer.

Dijkstra, H. A. and C. A. Katsman, 1997: Temporal variability of the wind-driven quasi-geostrophic double gyre ocean circulation: Basic bifurcation diagrams. Geophys. Astrophys. Fluid Dyn., 85, 195-232.

Dijkstra, H. A., M. J. Molemaker, A. V. der Ploeg, and E. F. F. Botta, 1995: An efficient code to compute nonparallel flows and their stability. Comp. Fluids, 24, 415-434.

Fantini, M. and K.-K. Tung, 1987: On radiating waves generated from barotropic shear instability of a western boundary current. J. Phys. Oceanogr., 17, 1304-1308.

Finley, C. A. and T. R. Nathan, 1993: On radiating baroclinic instability of zonally varying flow. Tellus, 45A, 54-71. 
Fox-Kemper, B., 2003: Eddies and friction: removing of vorticity from the winddriven gyre. Ph.D. thesis, MIT/WHOI Joint Program in Oceanography.

Fu, L.-L., B. Cheng, and B. Qiu, 2001: 25-day period large-scale oscillations in the Argentine basin revealed by the TOPEX/Poseidon altimeter. J. Phys. Oceanogr., 31, 506-517.

Fu, L. L. and B. Qui, 2002: Low-frequency variability of the North Pacific Ocean: The roles of boundary- and wind-driven baroclinic Rossby waves. J. Geophys. Res., 107(C12), 3220, doi:10.1029/2001JC001131.

Ghil, M., Y. Feliks, and L. Sushama, 2002: Baroclinic and barotropic aspects of the wind-driven ocean circulation. Physica D, 167, 1-35.

Gill, A. E., 1982: Atmosphere-Ocean Dynamics. Academic Press.

Haney, R. L., 1971: Surface thermal boundary condition for ocean circulation models. J. Phys. Oceanogr., 1, 241-248.

Held, I. M., 2000: The general circulation of the atmosphere. 2000 Program in Geophysical Fluid Dynamics Proceedings, Woods Hole.

Holland, W. R., 1978: The role of mesoscale eddies in the general circulation of the ocean: Numerical experiments using a wind-driven quasigeostrophic model. $J$. Phys. Oceanogr., 8, 363-392.

Holland, W. R. and P. R. Rhines, 1980: An example of eddy-induced ocean circulation. J. Phys. Oceanogr., 10, 1010-1031.

Huang, R. X., 1993: A two-level model for the wind- and buoyancy-forced circulation. J. Phys. Oceanogr., 23, 104-115.

Hughes, C. W., V. N. Stepanov, L.-L. Fu, B. Barnier, and G. W. Hargreaves, 2007: Three forms of variability in Argentine basin ocean bottom pressure. J. Geophys. Res., 112, 1464.

Ivanov, L. M., C. A. Collins, and T. M. Margolina, 2009: System of quasi-zonal jets off California revealed from satellite altimetry. Geophys. Res. Lett., 36, L03609, doi:10.1029/2008GL036327.

Jacobs, G. A., H. E. Hurlburt, and J. C. K. et al., 1994: Decade-scale trans-Pacific propagation and warming effects of an El Niño anomaly. Nature, 370, 360-363.

Jayne, S. R. and N. G. Hogg, 1999: On recirculation forced by an unstable jet. J. Phys. Oceanogr., 29, 2711-2718.

Jensen, V. G., 1959: Viscous flow round a sphere at low Reynolds numbers. Proc. Roy. Soc. London, A249, 346-366. 
Kamenkovich, I., P. Berloff, and J. Pedlosky, 2009: Role of eddy forcing in the dynamics of multiple zonal jets in the North Atlantic. J. Phys. Oceanogr., in press.

Kamenkovich, I. and J. Pedlosky, 1996: Radiating instabilities of nonzonal ocean currents. J. Phys. Oceanogr., 26, 622-643.

Le Traon, P. Y. and R. Morrow, 2000: Ocean currents and eddies. Satellite altimetry and earth sciences: a handbook of techniques and applications, L.-L. Fu and A. Cazenave, Eds., Academic Press, chap. 3.

Lozier, M. S. and S. C. Riser, 1989: Potential vorticity dynamics of boundary currents in a quasi-geostrophic ocean. J. Phys. Oceanogr., 19, 1373-1396.

Luyten, J. and H. Stommel, 1986: Gyres driven by combined wind and buoyancy flux. J. Phys. Oceanogr., 16, 1551-1560.

Maximenko, N. and P. Niiler, 2006: Mean surface circulation of the global ocean inferred from satellite altimeter and drifter data. 15 years of Progress in Radar Altimetry, Venice, Italy, European Space Agency, SP-614, URL http://earth.esa.int/cgi-bin/confalt15y.pl?abstract=780.

Maximenko, N. A., B. Bang, and H. Sasaki, 2005: Observational evidence of alternating zonal jets in the world ocean. Geophys. Res. Lett., 32, L12607, doi:10.1029/2005GL022728.

Maximenko, N. A., O. V. Melnichenko, P. P. Niiler, and H. Sasaki, 2008: Stationary mesoscale jet-like features in the oceans. Geophys. Res. Lett., 35, L08603, doi: 10.1029/2008GL033267.

McCalpin, J. D. and D. B. Haidvogel, 1996: Phenomenology of the low-frequency variability in a reduced-gravity quasi-geostrophic double-gyre model. J. Phys. Oceanogr., 26, 739-752.

McIntyre, M. E. and M. A. Weissman, 1978: On radiating instabilities and resonant overreflections. J. Atmos. Sci., 35, 1190-1196.

Munk, W., 1966: Abyssal recipes. Deep Sea Res., 13, 707-730.

Nauw, J. J., H. A. Dijkstra, and E. Simonnet, 2004: Regimes of low-frequency variability in a three-layer quasi-geostrophic ocean model. J. Mar. Res., 62, 685-720.

O'Dwyer, J. and R. G. Williams, 1997: The climatological distribution of potential vorticity over the abyssal ocean. J. Phys. Oceanogr., 27, 2488-2506.

Pedlosky, J., 1965: A study of the time-dependent ocean circulation. J. Atmos. Sci., 22, 267-272.

Pedlosky, J., 1987: Geophysical Fluid Dynamics. corr. 2nd ed., Springer. 
Pedlosky, J., 1998: Ocean Circulation Theory. corr. 2nd ed., Springer.

Pedlosky, J., 2002: The destabilization of Rossby normal modes by meridional baroclinic shear. J. Phys. Oceanogr., 32, 2418-2423.

Pedlosky, J., 2006: Time-dependent response to cooling in a beta-plane basin. J. Phys. Oceanogr., 36, 2185-2198.

Pedlosky, J. and M. A. Spall, 2005: Boundary intensification of vertical velocity in a beta-plane basin. J. Phys. Oceanogr., 35, 2487-2500.

Pierini, S., 2005: A model study of the spectral structure of boundary-driven Rossby waves and related altimetric implications. J. Phys. Oceanogr., 35, 218-231.

Pierrehumbert, R. T., 1984: Local and global baroclinic variability of zonally varying flow. J. Atmos. Sci., 41, 2141-2162.

Primeau, F., 1998: Multiple equilibria and low-frequency variability of wind-driven ocean models. Ph.D. thesis, MIT/WHOI Joint Program in Oceanography.

Rhines, P. B. and W. R. Young, 1982: A theory of the wind-driven circulation. i. mid-ocean gyres. J. Mar. Res., 40 (Suppl.), 559-596.

Richards, K. J., N. A. Maximenko, F. O. Bryan, and H. Sasaki, 2006: Zonal jets in the Pacific Ocean. Geophys. Res. Lett., 33, L03 605, doi:10.1029/2005GL024645.

Salmon, R., 1998: Lectures on Geophysical Fluid Dynamics. Oxford University Press, New York, Oxford.

Schlax, M. G. and D. B. Chelton, 2008: The influence of mesoscale eddies on the detection of quasi-zonal jets in the ocean. Geophys. Res. Lett., 35, L24602, doi: 10.1029/2008GL035998.

Schopp, R., 1993: Multiple equilibria for cross-gyre flow between subpolar and subtropical gyres. J. Phys. Oceanogr., 23, 1754-1766.

Simonnet, E., 2005: Quantization of the low-frequency variability of the double-gyre circulation. J. Phys. Oceanogr., 35, 2268-2290.

Simonnet, E. and H. A. Dijkstra, 2002: Spontaneous generation of low-frequency modes of variability in the wind-driven ocean circulation. J. Phys. Oceanogr., 32, $1747-1762$.

Smith, R. L., A. Huyer, J. S. Godfrey, and J. A. Church, 1991: The Leeuwin current off western Australia, 1986-1987. J. Phys. Oceanogr., 21, 323-345.

Spydell, M. and P. Cessi, 2003: Baroclinic modes in a two-layer basin. J. Phys. Oceanogr., 33, 610-622. 
Stanev, E. V. and N. H. Rachev, 1999: Numerical study of the planetary Rossby modes in the Black Sea. J. Marine Syst., 21, 238-306.

Stanev, E. V. and J. V. Staneva, 2000: The impact of the baroclinic eddies and the basin oscillations on the transitions between different quasi-stable states of the Black Sea circulation. J. Marine Syst., 24, 3-26.

Stommel, H. and A. B. Arons, 1960: On the abyssal circulation of the world ocean. i. stationary planetary flow patterns on a sphere. Deep Sea Res., 6, 140-154.

Talley, L. D., 1983a: Radiating barotropic instabilities. J. Phys. Oceanogr., 13, 972987.

Talley, L. D., 1983b: Radiating instabilities of a thin baroclinic jet. J. Phys. Oceanogr., 13, 2161-2181.

Terradas, J., R. Oliver, and J. L. Ballester, 2004: Application of statistical techniques to the analysis of solar coronal oscilations. The Astrophysical Journal, 614, 435447.

Verron, J. and E. Blayo, 1996: The no-slip condition and separation of western boundary currents. J. Phys. Oceanogr., 26, 1938-1952.

von Storch, H. and F. W. Zwiers, 1999: Statistical analysis in climate research. Cambridge Univ. Press, Cambridge.

Walker, A. and J. Pedlosky, 2002: Instability of meridional baroclinic currents. J. Phys. Oceanogr., 32, 1075-1093.

Warren, B. A., T. W. III, and J. H. LaCasce, 2002: Forced resonant undulations in the Mascarene basin. Deep-Sea Res. II, 49, 1513-1526.

Weatherly, G. L., 1993: On deep-current and hydrographic observations from a mudwave region and elsewhere in the Argentine basin. Deep-Sea Res. II, 40, 939-961.

Weijer, W., 2008: Normal modes of the Mascarene basin. Deep-Sea Res. I, 55, 128136.

Weijer, W., F. Vivier, S. T. Gille, and H. A. Dijkstra, 2007a: Multiple oscillatory modes of the Argentine basin. Part I: statistical analysis. J. Phys. Oceanogr., 37, $2855-2868$.

Weijer, W., F. Vivier, S. T. Gille, and H. A. Dijkstra, 2007b: Multiple oscillatory modes of the Argentine basin. Part II: the spectral origin of basin modes. J. Phys. Oceanogr., 37, 2869-2881. 